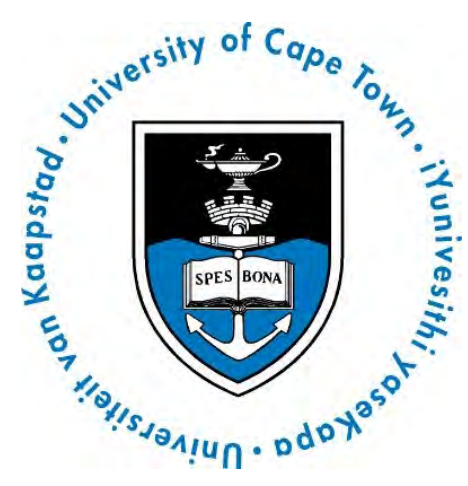

\title{
Assessment of the Potential Carbon Footprint of Engineered Processes for the Mineral Carbonation of PGM Tailings
}

\author{
Thesis presented to the University of Cape Town \\ in the fulfilment of the requirements \\ for the degree of
}

Master of Science in Chemical Engineering

by

Mpendulo S. Ncongwane

May 2016 
The copyright of this thesis vests in the author. No quotation from it or information derived from it is to be published without full acknowledgement of the source. The thesis is to be used for private study or noncommercial research purposes only.

Published by the University of Cape Town (UCT) in terms of the non-exclusive license granted to UCT by the author. 
I am aware that plagiarism (the use of someone else's work without their permission and/or without acknowledging the original source) is wrong.

I confirm that this work is my own unaided work, except where it has been explicitly acknowledged otherwise.

I have followed the required conventions in referencing the thoughts and ideas of others.

\section{Signed by candidate}




\begin{abstract}
Mineral carbonation is a carbon sequestration technology that entails the reaction of $\mathrm{CO}_{2}$ with oxides or silicates of magnesium, calcium or iron to produce stable carbonate compounds. Magnesium-rich tailings from the platinum industry in South Africa have been identified as a potentially viable and attractive feedstock for $\mathrm{CO}_{2}$ sequestration through mineral carbonation. Many of the strategies proposed to enhance the dissolution kinetics of silicate minerals, such as the use of elevated temperatures and pressures and chemical additives, as well as pretreatment through mechanical and thermal activation, are energy intensive and will thus reduce the net $\mathrm{CO}_{2}$ sequestration capacity of the overall mineral carbonation process. As a result, there is growing recognition of the need to evaluate the processes using life-cycle based approaches and tools to ensure they result in net $\mathrm{CO}_{2}$ reduction. However, to date, research and development has focused primarily on the optimisation of extraction and/or carbonation efficiencies, with specific emphasis on the relatively reactive silicate minerals, such as olivine and serpentine.
\end{abstract}

This project seeks to investigate the viability of using pyroxene-rich PGM tailings for the sequestration of $\mathrm{CO}_{2}$, with specific emphasis on net carbon neutrality. Promising mineral carbonation processes have been identified on the basis of an extensive literature review, and include the: ammonium salts pH swing, Lackner's $\mathrm{HCl}$ multi-stage, gas-solid Åbo Akademi University process, direct aqueous process, and mineral acid $\mathrm{pH}$ swing. Material and energy balances were then conducted for these processes on the basis of the sequestration of 1 ton of carbon dioxide, using Aspen Plus v8 simulation software package. The material and energy data were then used to determine the total carbon footprint contributions, through the use of SimaPro v 7.7.3. life cycle assessment software.

The selected carbonation processes were found to release more carbon dioxide than the process sequesters. The carbonation process resulting in the most emissions released was found to be Lackner's multi-stage process (18 $\left.295 \mathrm{~kg}-\mathrm{CO}_{2} \mathrm{e}\right)$, followed by the ammonium salts process $\left(8798 \mathrm{~kg}-\mathrm{CO}_{2} \mathrm{e}\right.$ ), per ton carbon dioxide sequestered. The carbonation process resulting in the least emissions released was the solid Åbo Akademi University process (1354 kg- $\left.\mathrm{CO}_{2} \mathrm{e}\right)$, followed by the gas-solid direct aqueous process (2 $\left.364 \mathrm{~kg}-\mathrm{CO}_{2} \mathrm{e}\right)$, as well as the mineral acid $\mathrm{pH}$ swing process ( $3126 \mathrm{~kg}-\mathrm{CO}_{2} \mathrm{e}$ ). The most carbon emissions intensive contributions to the carbon footprint were found to be heat requirements and chemical reagent make-up, which generally accounted for more than $85 \%$ of total emissions when combined. 
Aqueous processes generally incurred a much higher carbon footprint, despite using relatively lower temperatures than the gas-solid ÅAU process. This was attributed to the higher quantities of water used in the aqueous processes that, in some cases, were subject to phase change via, for example, evaporation. Additionally, the production of make-up chemical reagent, alone, was found to result in emissions that exceeded the carbon dioxide sequestered for four of the five selected processes (ammonium salts process, Lackner's $\mathrm{HCl}$ multi-stage, direct aqueous, mineral acid $\mathrm{pH}$ swing). The potential to reduce emissions associated with heat generation could be achieved through the exploration of heat integration and cleaner alternative sources of heat, for the potentially feasible processes. On the other hand, the carbon dioxide emissions associated with make-up reagent could be reduced through the use of cleaner input materials as well as by increasing the recycle ratios to reduce external reagent requirement. 


\section{Acknowledgements}

I would like to express my outmost gratitude to my supervisor Dr. Jennifer Broadhurst and my co-supervisor Prof. Jochen Petersen, for the constant and unwavering support they have offered me throughout the course of this thesis. I am a better researcher, better thinker and better human because of the guidance, advice and motivation I received as I took on this exciting challenge. I am indebted to you.

A special word of appreciation to:

- Prof J.P. Franzidis and Minerals to Metals for granting me the opportunity to explore my scientific curiosity at the Initiative.

- Hilton Heydenrych, Dr. Siew Tai, Sibongiseni and Derrick for assisting me with troubleshooting Aspen Plus, and giving me invaluable tips on how to deal with certain aspects of the software. Without you, this work would certainly have been impossible.

- Mymoena van der Fort, Belinda Davids and Eunice Jacobs for their assistance with administrative matters.

- Kyle Hauslaib for assisting me with the installation of Aspen Plus, and Makhosazane "Chucky” Kunene with SimaPro.

- My friends and colleagues from UCT with whom I have had the fortune and pleasure to share moments, struggles and experiences.

- My dearest family and friends, for the love, the support, and belief that you have shown to me throughout my studies. It is what kept me going. A very special mention to my big sister Dudu, you are the best.

- Finally, all the individuals that have helped carve my character and intellect through simple conversations and interactions.

To my late parents, Sam and Constance, and my dearest sister Ruth, this is for you.

May your souls rest in everlasting peace. 


\section{Table of Contents}

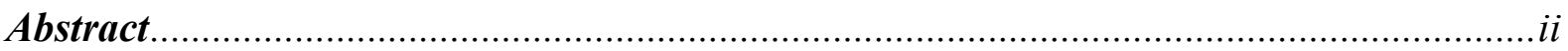

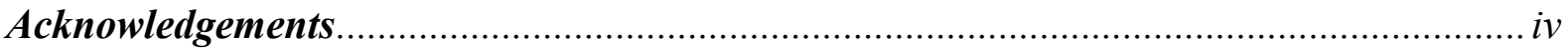

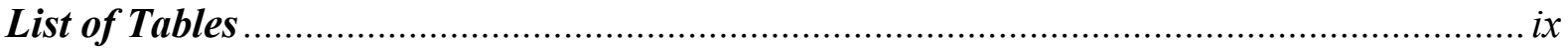

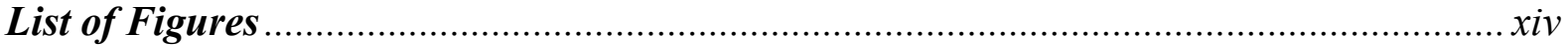

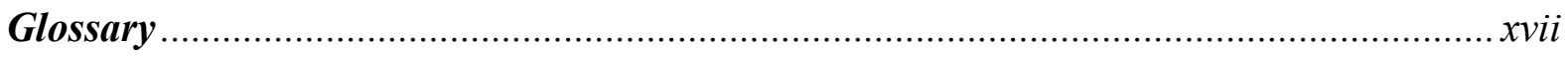

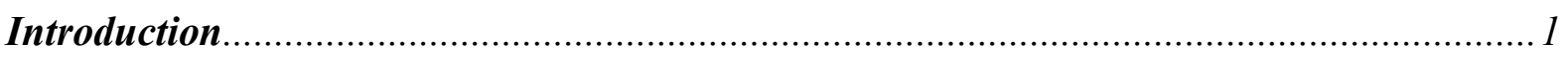

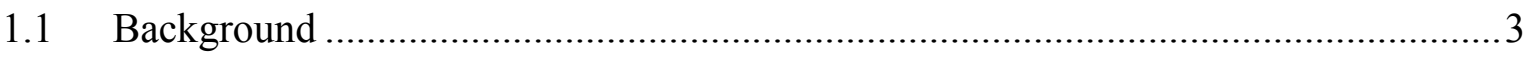

1.1.1 Engineered Mineral Carbonation Processes …............................................. 3

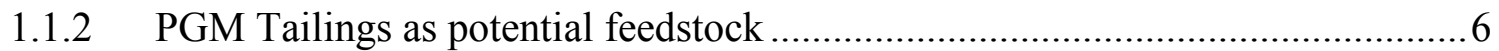

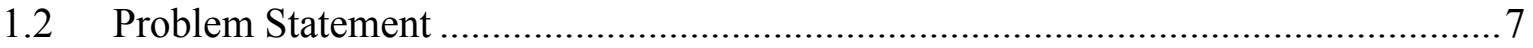

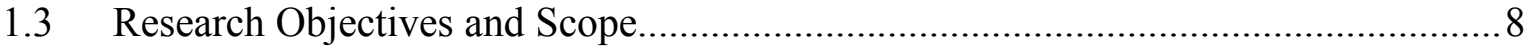

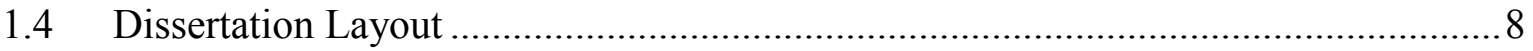

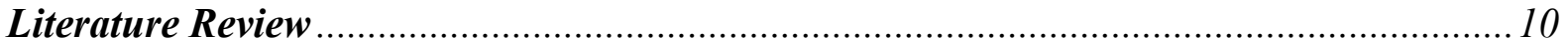

$2.1 \quad$ Engineered Mineral Carbonation Approaches …................................................... 10

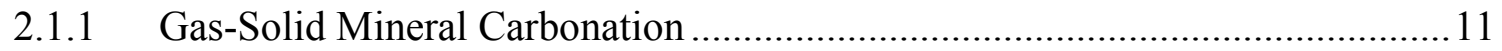

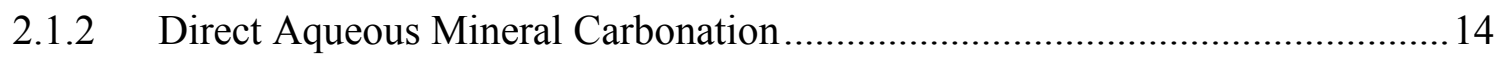

2.1.3 Indirect Aqueous Mineral Carbonation .......................................................... 19

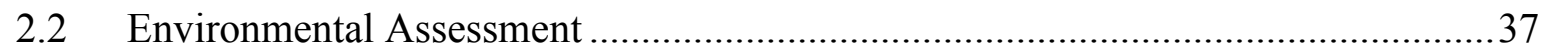

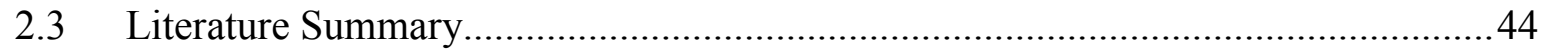

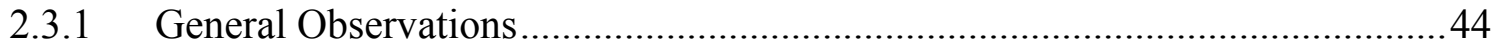

2.3.2 Summary and Comparison of Mineral Carbonation Processes ........................45

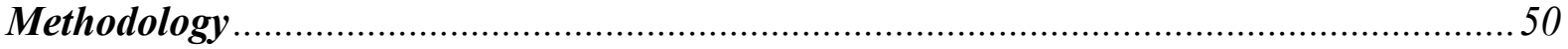

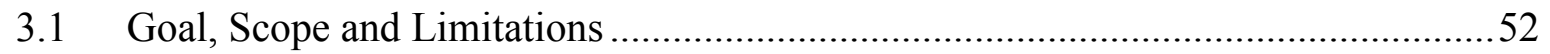

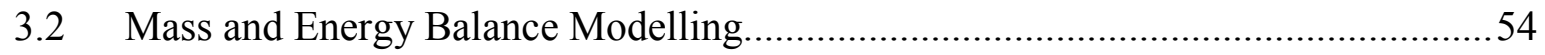

3.2.1 General Assumptions and Approach for Model Development..........................54

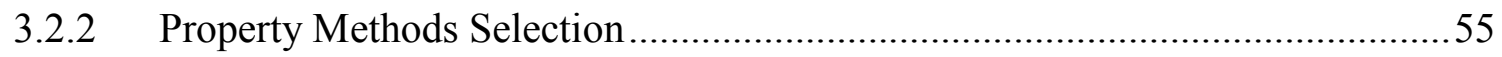


3.2.3 Simulation Development Illustrations

3.3 Carbon Dioxide Emissions Accounting ....................................................................58

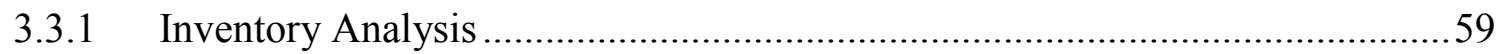

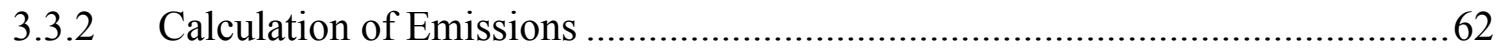

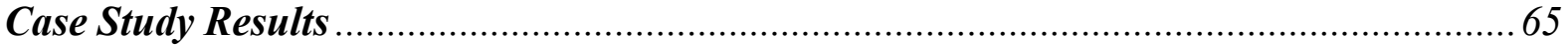

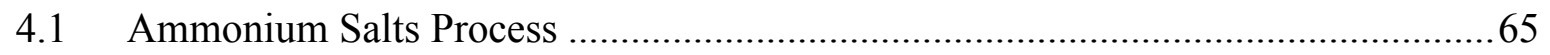

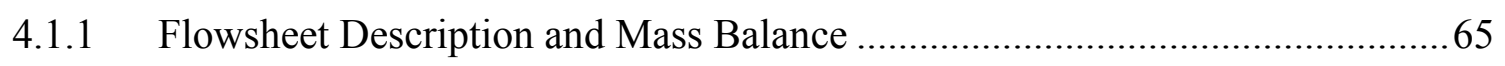

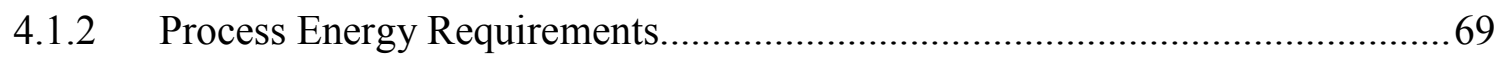

4.1.3 Carbon Dioxide Footprint ........................................................................... 71

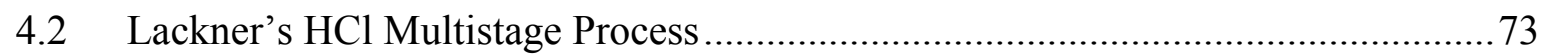

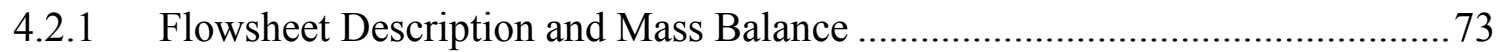

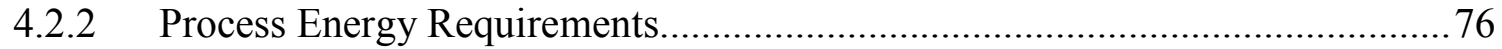

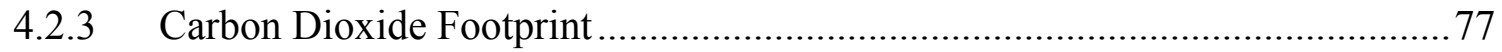

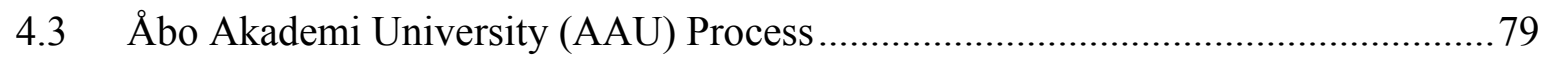

4.3.1 Flowsheet Description and Mass Balance …............................................... 79

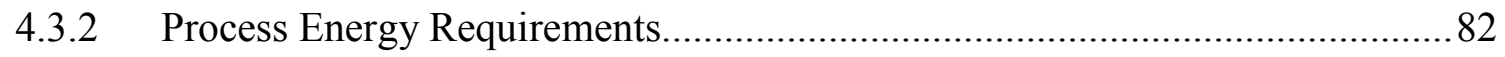

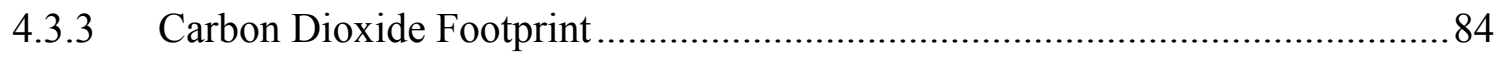

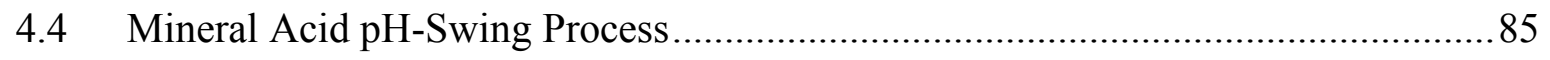

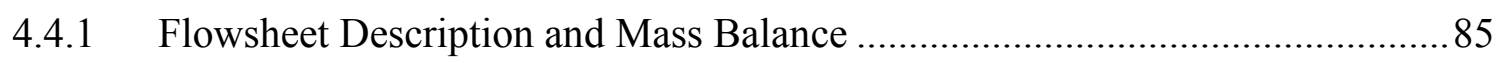

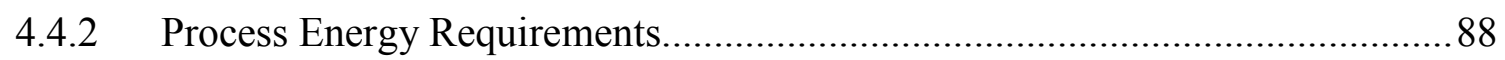

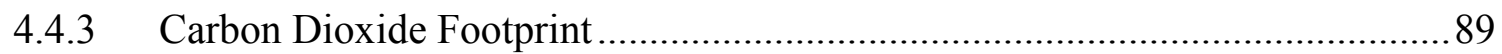

4.5 Direct Aqueous Carbonation Process.................................................................... 91

4.5.1 Flowsheet Description and Mass Balance ................................................... 91

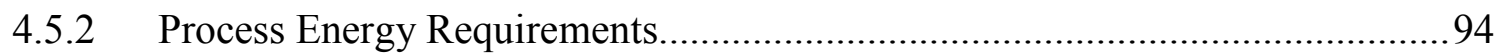

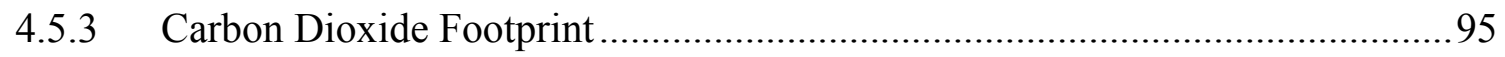

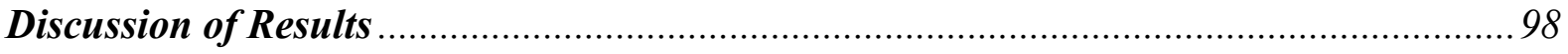

5.1 Mineral Carbonation Process Energy Comparisons.............................................98 


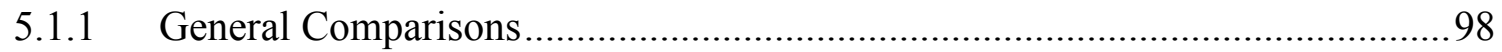

5.1.2 Comparisons between Aqueous Processes …................................................ 100

5.2 Mineral Carbonation Carbon Dioxide Footprint Comparisons ................................. 101

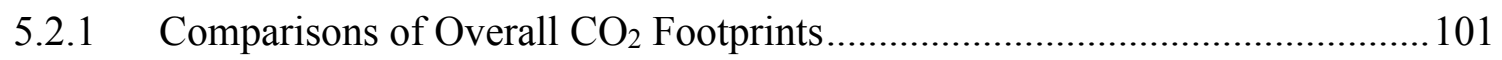

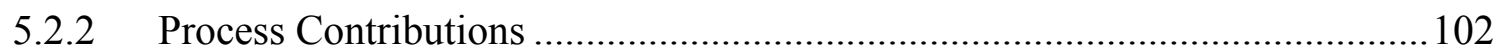

5.3 The Potential Viability of Selected Mineral Carbonation Processes ...................... 104

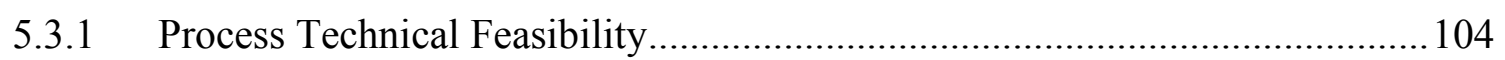

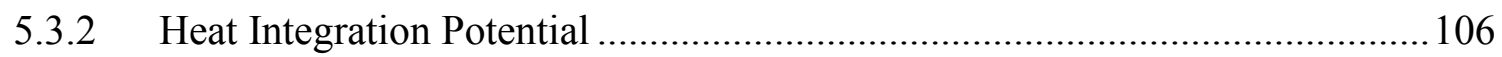

5.3.3 Additional Process Impacts and Plant Construction........................................ 107

5.3.4 Chemical Reagent Recovery, Recycles and Losses...................................... 107

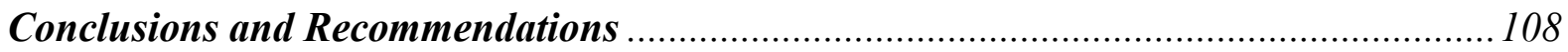

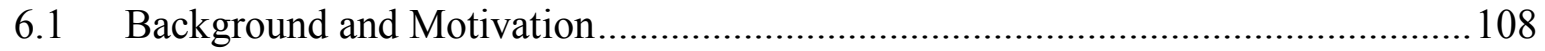

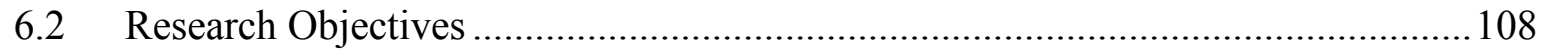

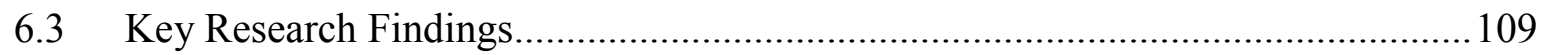

6.3.1 Identification of Potentially Feasible Mineral Carbonation Processes ............ 109

6.3.2 Material and Energy Balance Simulations.................................................. 109

6.3.3 Mineral Carbonation Process Sustainability - Effectiveness to Sequester $\mathrm{CO}_{2} \ldots . .110$

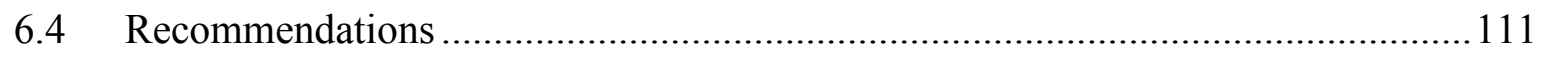

6.4.1 Reducing heating related carbon emissions ................................................ 112

6.4.2 Reducing material and chemical related carbon emissions ............................ 113

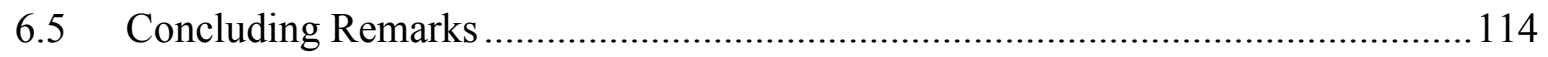

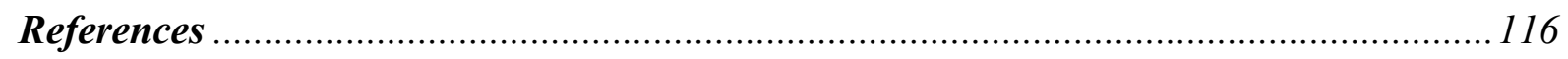

Appendix A: Initialization Data for Aspen Plus Simulations .................................... 125

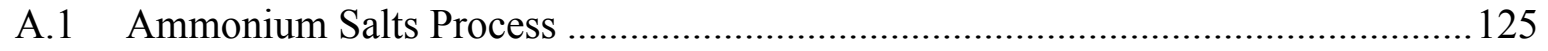

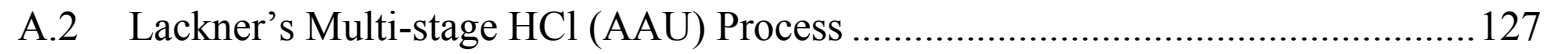

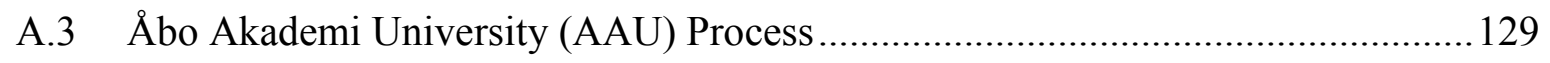

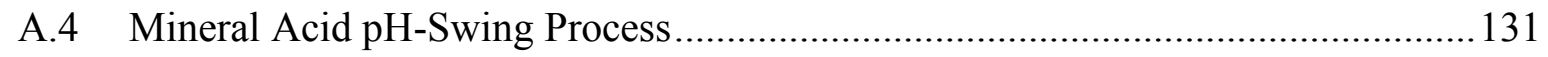


A.5 Direct Aqueous Carbonation Process.................................................................... 132

Material Balances for Mineral Carbonation Processes ................................................ 134

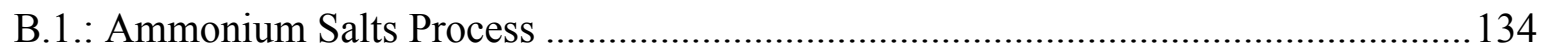

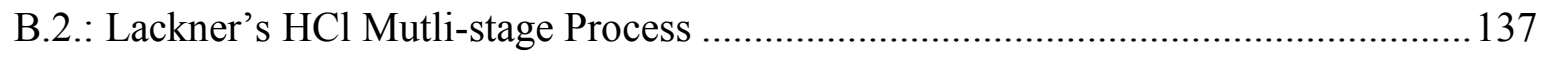

B.3.: Åbo Akademi University (AAU) Process ........................................................... 140

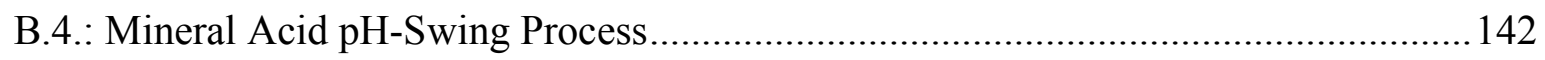

B.5.: Direct Aqueous Carbonation Process............................................................... 145

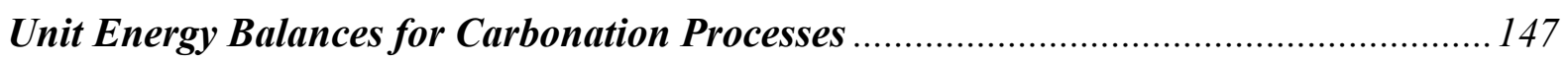

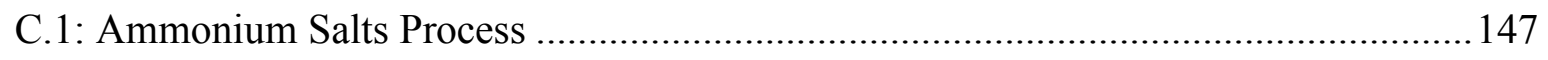

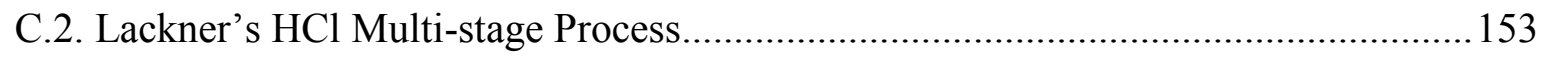

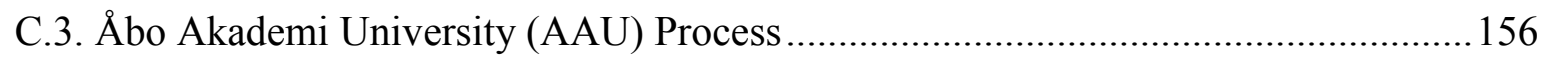

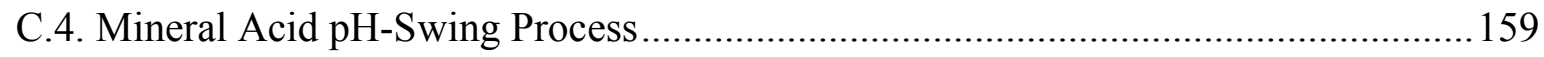

C.5. Direct Aqueous Carbonation Process..................................................................... 162

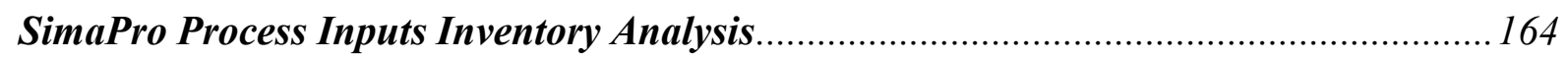

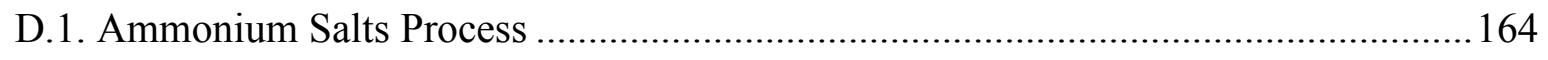

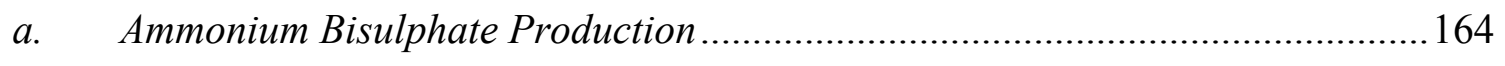

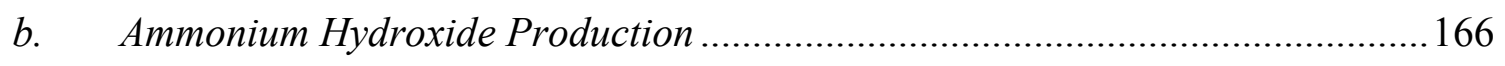

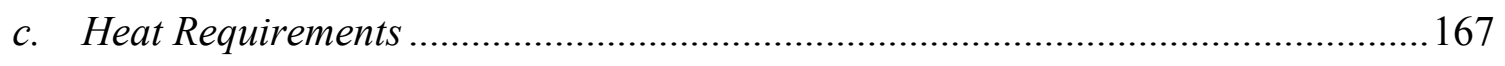

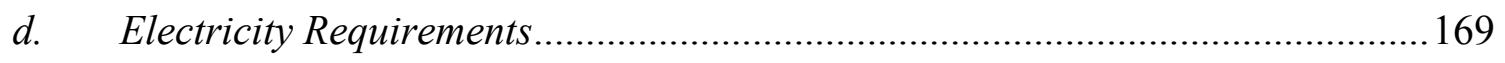

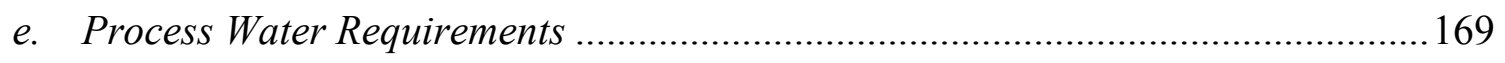

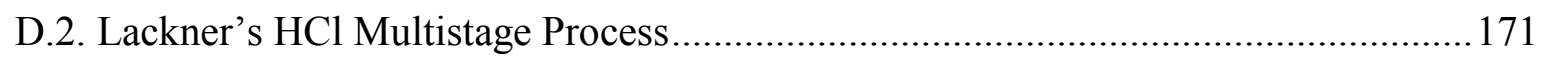

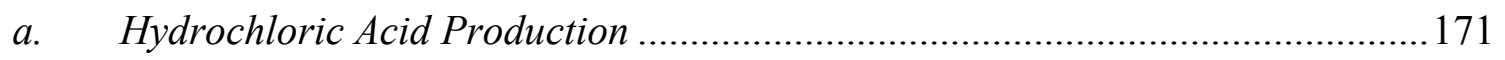

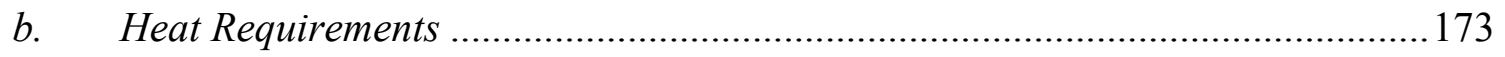

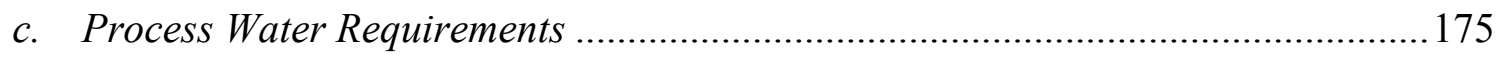

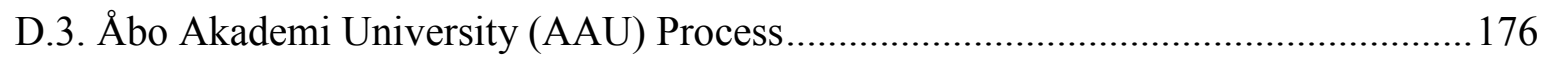

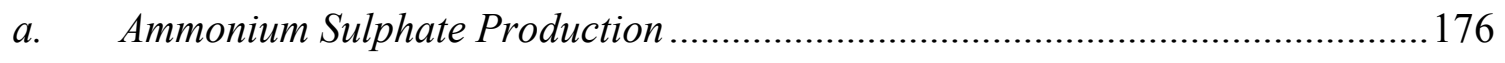

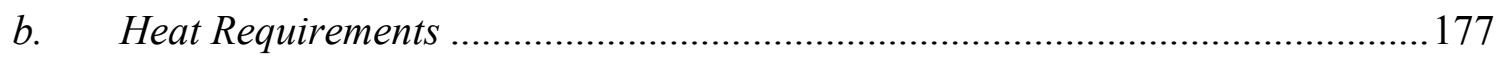




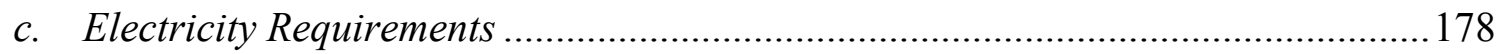

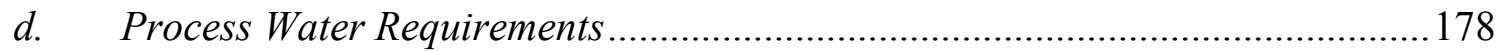

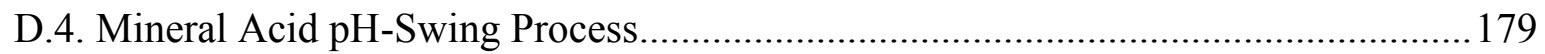

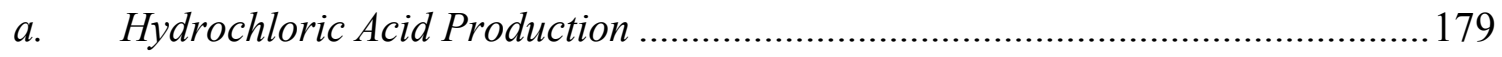

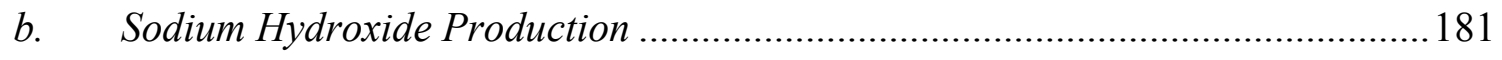

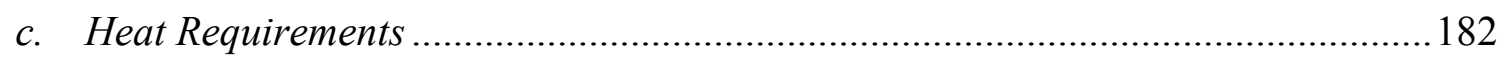

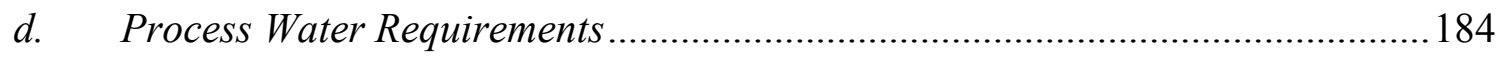

D.5. Direct Aqueous Carbonation Process ................................................................... 186

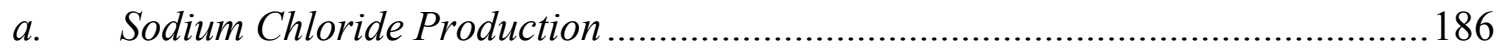

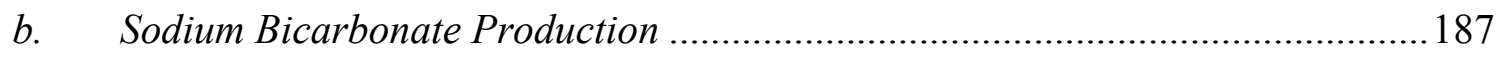

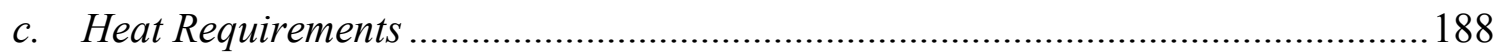

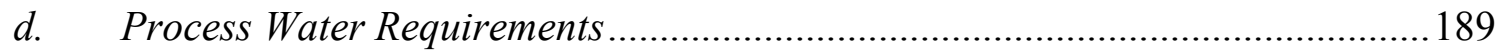

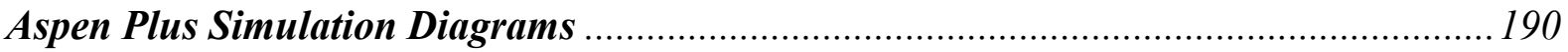

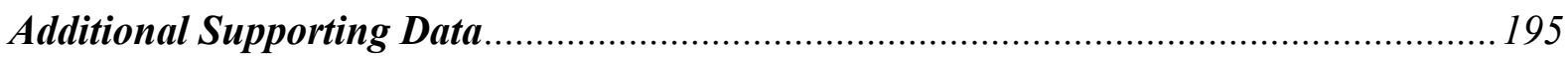

\section{List of Tables}

Table 2-1: Direct carbonation based theoretical capacities of silicate minerals to sequester

$\mathrm{CO}_{2}$ (Meyer et al., 2014)

Table 2-2: Conversion of $\mathrm{Mg}(\mathrm{OH})_{2}$ to $\mathrm{MgCO}_{3}$ at various temperature/pressure conditions after 6 hrs (Teir et al., 2006)

Table 2-3: Optimum carbonation conditions, by mineral (Adapted from Gerdemann et al.,

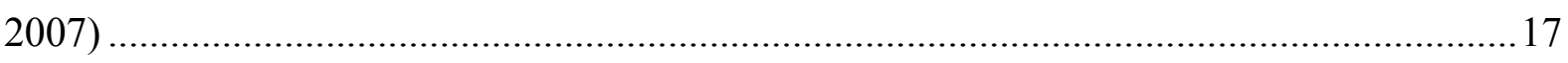

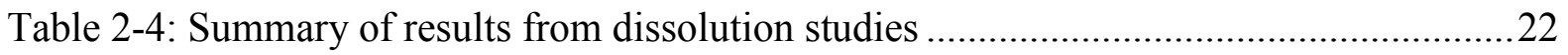

Table 2-5: Impact of mineral type on magnesium extraction under the same conditions .......27

Table 2-6: Selected carbonation processes from literature (Newall et al., 2000) ...................38

Table 2-7: Energy burdens for the NETL mineral carbonation route (O'Connor et al., 2005) 39

Table 2-8: Summary of selected processes and their key attributes ......................................49

Table 3-1: Global Warming Potentials (GWP100) for greenhouse gases (IPCC, 2007).........63

Table 4-1: Chemical reactions occurring in the ammonium salts process.............................66 
Table 4-2: Material requirements for ammonium salts carbonation 69

Table 4-3: Process contributions to carbon dioxide footprint $\left(\mathrm{kg}-\mathrm{CO}_{2} \mathrm{e}\right)$............................... 71

Table 4-4: Chemical reactions occurring in Lackner's HCl multi-stage process.....................74

Table 4-5: Material requirements for Lackner's $\mathrm{HCl}$ multi-stage process.............................76

Table 4-6: Process contributions to carbon dioxide footprint $\left(\mathrm{kg}-\mathrm{CO}_{2} \mathrm{e}\right)$............................. 78

Table 4-7: Key reactions occurring in the Åbo Akademi University process .........................80

Table 4-8: Material requirements for Åbo Akademi University process ...............................82

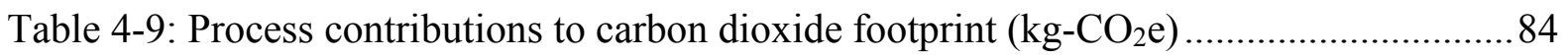

Table 4-10: Key reactions occurring in the mineral acid $\mathrm{pH}$-Swing process .........................86

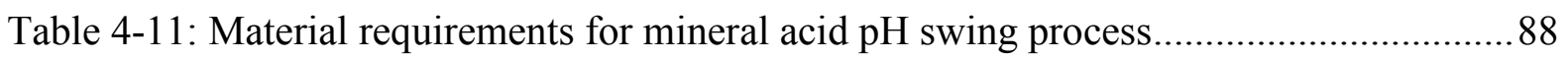

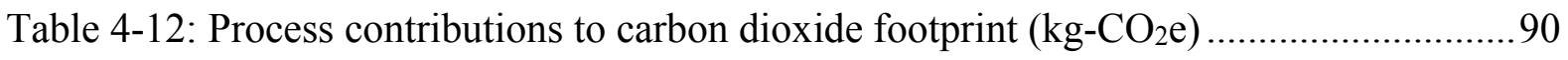

Table 4-13: Chemical reactions occurring in the direct aqueous carbonation process............92

Table 4-14: Material requirements for direct aqueous carbonation process ..........................94

Table 4-15: Process contributions to carbon dioxide footprint $\left(\mathrm{kg}-\mathrm{CO}_{2} \mathrm{e}\right) \ldots \ldots \ldots \ldots \ldots \ldots \ldots \ldots \ldots . . . . . . . . . . . . . .66$

Table 5-1: Comparison between feed water and process energy requirements of indirect aqueous processes

Table 5-2: Percentage contributions of process requirements to total carbon dioxide emissions

Table 5-3: Carbon dioxide emissions for chemical reagent make-up................................. 103

Table A-1: Initialization data for Aspen modelling of ammonium salts process .................. 125

Table A-2: Initialization data for Aspen Modelling of Lackner's multi-stage process .......... 127

Table A-3: Initialization data for Aspen Modelling of Åbo Akademi University process.... 129

Table A-4: Initialisation data for Aspen model of mineral acid $\mathrm{pH}$ swing process...............131

Table A-5: Initialisation data for Aspen model of direct aqueous process ............................. 132

Table C-1: Mineral dissolution unit energy balance for 30\% extraction.............................. 147

Table C-2: $\mathrm{pH}$ adjustment unit energy balance for 30\% extraction.................................... 148

Table C-3: Mineral carbonation unit energy balance for 30\% extraction ............................ 149

Table C-4: $\mathrm{CO}_{2}$ capture unit energy balance for $30 \%$ extraction ...................................... 150

Table C-5: Thermal decomposition (EVAP) unit energy balance for 30\% extraction.......... 151

Table C-6: Mineral carbonation unit energy balance for $90 \%$ dissolution ........................... 152

Table C-7: Mineral dissolution unit energy balance for 30\% extraction.............................. 153

Table C-8: Conversion and repartition unit energy balance for 30\% extraction ................... 154

Table C-9: Mineral carbonation unit energy balance for 30\% extraction .......................... 155 
Table C-10: Mg-extraction (AS-REAC) unit energy balance for $66 \%$ extraction and $55 \%$ carbonation

Table C-11: Precipitation unit energy balance for $66 \%$ extraction and 55\% carbonation..... 157

Table C-12: Mineral carbonation unit energy balance for $66 \%$ extraction and 55\% carbonation.

Table C-13: Mineral dissolution unit energy balance for $20 \%$ extraction and $65 \%$ carbonation

Table C-14: $\mathrm{pH}$ adjustment unit energy balance for $20 \%$ extraction and $65 \%$ carbonation.. 160

Table C-15: Mineral carbonation unit energy balance for 20\% extraction and $65 \%$ carbonation

Table C-16: Direct aqueous carbonation for $5 \%$ carbonation

Table C-17: Direct aqueous carbonation for $20 \%$ carbonation

Table D-1: SimaPro results of carbon dioxide burdens of ammonium bisulphate production at $30 \%$ extraction

Table D-2: SimaPro results of carbon dioxide burdens of ammonium bisulphate production at $50 \%$ extraction

Table D-3: SimaPro results of carbon dioxide burdens of ammonium bisulphate production at $90 \%$ extraction

Table D-4: SimaPro results of carbon dioxide burdens of ammonium hydroxide production at $30 \%$ extraction 166

Table D-5: SimaPro results of carbon dioxide burdens of ammonium hydroxide production at $50 \%$ extraction

Table D-6: SimaPro results of carbon dioxide burdens of ammonium hydroxide production at $90 \%$ extraction

Table D-7: SimaPro results of carbon dioxide burdens of process heat requirements at $30 \%$ extraction

Table D-8: SimaPro results of carbon dioxide burdens of process heat requirements at $50 \%$ extraction

Table D-9: SimaPro results of carbon dioxide burdens of process heat requirements at $90 \%$ extraction

Table D-10: SimaPro results of carbon dioxide burdens of compression electricity requirements (All extraction efficiencies)

Table D-11: SimaPro results of carbon dioxide burdens of process water requirements at $30 \%$ extraction 
Table D-12: SimaPro results of carbon dioxide burdens of process water requirements at $50 \%$ extraction

Table D-13: SimaPro results of carbon dioxide burdens of process water requirements at $90 \%$ extraction.

Table D-14: SimaPro results of carbon dioxide burdens of hydrochloric acid production at $30 \%$ extraction

Table D-15: SimaPro results of carbon dioxide burdens of hydrochloric acid production at $50 \%$ extraction

Table D-16: SimaPro results of carbon dioxide burdens of hydrochloric acid production at $90 \%$ extraction

Table D-17: SimaPro results of carbon dioxide burdens of process heat requirements at $30 \%$ extraction

Table D-18: SimaPro results of carbon dioxide burdens of process heat requirements at $50 \%$ extraction

Table D-19: SimaPro results of carbon dioxide burdens of process heat requirements at $90 \%$ extraction

Table D-20: SimaPro results of carbon dioxide burdens of process water requirements at $30 \%$ extraction

Table D-21: SimaPro results of carbon dioxide burdens of process water requirements at $50 \%$ extraction

Table D-22: SimaPro results of carbon dioxide burdens of process water requirements at $90 \%$ extraction

Table D-23: SimaPro results of carbon dioxide burdens of ammonium bisulphate production (All extraction efficiencies)

Table D-24: SimaPro results of carbon dioxide burdens of process heat requirements at $66 \%$ extraction and $55 \%$ carbonation

Table D-25: SimaPro results of carbon dioxide burdens of process heat requirements at $100 \%$ extraction and $80 \%$ carbonation

Table D-26: SimaPro results of carbon dioxide burdens of compression electricity requirements (All extraction efficiencies)

Table D-27: SimaPro results of carbon dioxide burdens of process water requirements (All extraction efficiencies)

Table D-28: SimaPro results of carbon dioxide burdens of hydrochloric acid production at $20 \%$ extraction and $65 \%$ carbonation 
Table D-29: SimaPro results of carbon dioxide burdens of hydrochloric acid production at $30 \%$ extraction and $90 \%$ carbonation

Table D-30: SimaPro results of carbon dioxide burdens of hydrochloric acid production at $50 \%$ extraction and $90 \%$ carbonation

Table D-31: SimaPro results of carbon dioxide burdens of hydrochloric acid production (All Cases)

Table D-32: SimaPro results of carbon dioxide burdens of process heat requirements at $20 \%$ extraction and $65 \%$ carbonation

Table D-33: SimaPro results of carbon dioxide burdens of process heat requirements at 30\% extraction and $90 \%$ carbonation

Table D-34: SimaPro results of carbon dioxide burdens of process heat requirements at $50 \%$ extraction and $90 \%$ carbonation.

Table D-35: SimaPro results of carbon dioxide burdens of process water requirements at $20 \%$ extraction and $65 \%$ carbonation

Table D-36: SimaPro results of carbon dioxide burdens of process water requirements at $30 \%$ extraction and $90 \%$ carbonation

Table D-37: SimaPro results of carbon dioxide burdens of process water requirements at $50 \%$ extraction and $90 \%$ carbonation.

Table D-38: SimaPro results of carbon dioxide burdens of sodium chloride requirements at $5 \%$ carbonation

Table D-39: SimaPro results of carbon dioxide burdens of sodium chloride requirements at $20 \%$ carbonation

Table D-40: SimaPro results of carbon dioxide burdens of sodium bicarbonate requirements at $5 \%$ carbonation

Table D-41: SimaPro results of carbon dioxide burdens of sodium bicarbonate requirements at $20 \%$ carbonation

Table D-42: SimaPro results of carbon dioxide burdens of heat requirements at 5\% carbonation.

Table D-43: SimaPro results of carbon dioxide burdens of heat requirements at $20 \%$ carbonation.

Table D-44: SimaPro results of carbon dioxide burdens of process water requirements at $5 \%$ carbonation

Table D-45: SimaPro results of carbon dioxide burdens of process water requirements at $20 \%$ carbonation. 
Table F-1: Net electricity requirements of selected mineral carbonation processes 195

Table F-2: Process carbon dioxide emissions burdens and contributions 195

Table F-3: The overall conversion of pyroxene in the selected mineral carbonation processes

\section{List of Figures}

Figure 1-1: Carbon capture and storage overview schematic (CO2CRC)

Figure 1-2: Mineral carbonation process scheme (Global CCS Institute, 2011) ......................3

Figure 1-3: Classification of mineral carbonation process routes (adapted from Olajire, 2013)

Figure 1-4: Carbon dioxide emissions sites and ultramafic rock deposits in $300 \mathrm{~km}$ radius

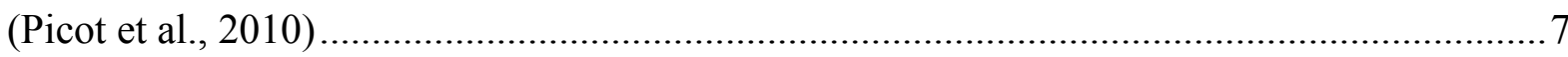

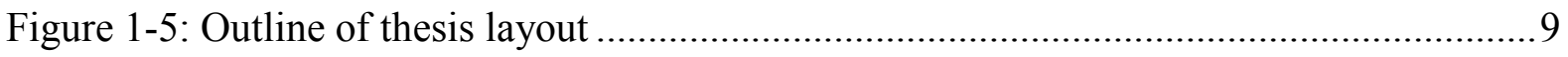

Figure 2-1: Schematic of Åbo Akademi University multi-stage process (adapted from Romao et al., 2012) .13

Figure 2-2: Thermodynamic equilibrium compositions for $\mathrm{Mg}_{2} \mathrm{SiO}_{4}-\mathrm{CO}_{2}-\mathrm{H}_{2} \mathrm{O}$ system

(Gerdemann et al., 2002) .16

Figure 2-3: The effect of pressure on the extent of carbonation (Gerdemann et al., 2002).....16

Figure 2-4: Extent of carbonation of olivine, wollastonite and serpentine at $185^{\circ} \mathrm{C}$ and 150 $\operatorname{atm} \mathrm{CO}_{2}$ in $\mathrm{NaCl} / \mathrm{NaHCO}_{3}$ solution (Gerdemann et al., 2007)............................................ 19

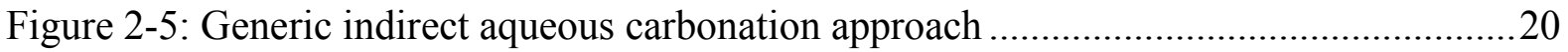

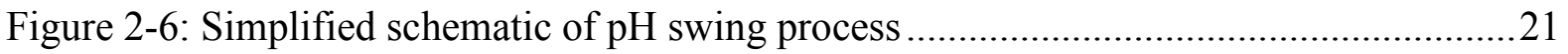

Figure 2-7: Extraction efficiency of magnesium from serpentinite in 1 M, 2 M, 4 M solutions at $20^{\circ} \mathrm{C}$ in $1 \mathrm{hr}$ (Teir et al., 2007a)

Figure 2-8: Extraction efficiency of magnesium from serpentinite in ammonium salt and sulphuric acid solutions at $70{ }^{\circ} \mathrm{C}$ in $3 \mathrm{hrs}$ (Wang and Maroto-Valer, 2011)

Figure 2-9: Effect of temperature on extent of dissolution of $\mathrm{Mg}$ and $\mathrm{Fe}$ in $2 \mathrm{M} \mathrm{H}_{2} \mathrm{SO}_{4}$ (Teir

et al., 2007a)

Figure 2-10: Effect of temperature on the dissolution of serpentine in $1.4 \mathrm{M} \mathrm{NH}_{4} \mathrm{HSO}_{4}$ solution (Wang and Maroto-Valer, 2011).

Figure 2-11: Effect of temperature on the dissolution of pyroxene in $1.4 \mathrm{M} \mathrm{NH}_{4} \mathrm{HSO}_{4}$ solution (Sanna et al., 2014a). 
Figure 2-12: Results from the conversion of magnesium ions into carbonate, effect of $\mathrm{pH}$ (Teir et al., 2007b)

Figure 2-13: A simple flowsheet for the carbonation of serpentinite through a $\mathrm{pH}$ swing method using $\mathrm{HCl} / \mathrm{HNO}_{3}$ and $\mathrm{NaOH}$ (Teir et al., 2009).

Figure 2-14: Process schematic of recyclable ammonium salts process (adapted from Wang and Maroto-Valer, 2013)

Figure 2-15: Simplified schematic of Lackner's multi-stage process

Figure 2-16: Relative free energy changes at the main process stages of the indirect carbonation (Teir, 2008)

Figure 2-17: Schematic of acetic acid based carbonation of wollastonite (Kakizawa et al., 2001)

Figure 2-18: $\mathrm{CO}_{2}$ emissions for the direct aqueous mineral carbonation processes as a function of feedstock and carbonation (Kirchofer et al., 2012)

Figure 2-19: Life cycle greenhouse gas emissions for mineral carbonation and geological storage in Europe in 2025 (Giannoulakis et al., 2014)

Figure 3-1: Research approach summary

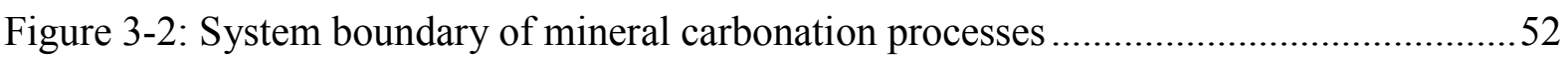

Figure 3-3: Chemical component input on Aspen Plus simulation software..........................57

Figure 3-4: Property method selection Tab on Aspen Plus simulation package ....................58

Figure 3-5: A representation of midpoint and endpoint approach to climate change

(Goedkoop et al., 2013)

Figure 4-1: Ammonium salts process material balance at 30\% extraction efficiency....

Figure 4-2: Energy requirements for major process units in the ammonium salts process .....70

Figure 4-3: $\mathrm{CO}_{2}$ footprint of material and energy requirements of ammonium salts process

$\left(\mathrm{kg}-\mathrm{CO}_{2} \mathrm{e}\right)$

Figure 4-4: Material balance for Lackner's multi-stage process for 30\% extraction efficiency

Figure 4-5: Energy requirements for major process units in the Lackner's $\mathrm{HCl}$ multi-stage process.

Figure 4-6: $\mathrm{CO}_{2}$ footprint of material and energy requirements of Lackner's $\mathrm{HCl}$ multi-stage process $\left(\mathrm{kg}-\mathrm{CO}_{2} \mathrm{e}\right)$

Figure 4-7: Material balance for Åbo Akademi University process for 60\% extraction efficiency and 55\% carbonation efficiency..... 
Figure 4-8: Energy requirements for major process units in the Åbo Akademi University process.

Figure 4-9: $\mathrm{CO}_{2}$ footprint of material and energy requirements of Åbo Akademi University process $\left(\mathrm{kg}-\mathrm{CO}_{2} \mathrm{e}\right)$

Figure 4-10: Material balance for mineral acid $\mathrm{pH}$ swing process for $20 \%$ extraction efficiency and $65 \%$ carbonation efficiency.

Figure 4-11: Energy requirements for major process units in the mineral acid $\mathrm{pH}$ swing process.

Figure 4-12: $\mathrm{CO}_{2}$ footprint of material and energy requirements of mineral acid $\mathrm{pH}$ swing process $\left(\mathrm{kg}-\mathrm{CO}_{2} \mathrm{e}\right)$

Figure 4-13: Material balance for direct aqueous carbonation process for 5\% carbonation efficiency.

Figure 4-14: Energy requirements for major process units in the direct aqueous carbonation process.

Figure 4-15: $\mathrm{CO}_{2}$ footprint of material and energy requirements of direct aqueous carbonation process $\left(\mathrm{kg}-\mathrm{CO}_{2} \mathrm{e}\right)$.

Figure 5-1: Comparison of process heat requirements of selected carbonation processes......98

Figure 5-2: Comparison of carbon dioxide footprints of selected carbonation process system 101

Figure E-1: Ammonium Salts process Aspen Plus simulation diagram 190

Figure E-2: Lackner's $\mathrm{HCl}$ process Aspen simulation diagram 191

Figure E-3: Åbo Akademi University process Aspen Plus simulation diagram. 192

Figure E-4: Mineral acid $\mathrm{pH}$ swing process Aspen simulation diagram .... 193

Figure E-5: Direct aqueous carbonation Aspen Plus simulation diagram 194 


\section{Glossary}

$\AA \mathrm{AU}$

AS

Aspen Plus

atm

Background

BP

BRPM

$\mathrm{Ca}$

$\mathrm{CaSiO}_{3}$

CCS

$\mathrm{CFC}$

$\mathrm{CH}_{4}$

$\mathrm{CH}_{3} \mathrm{COOH}$

$\mathrm{CH}_{3} \mathrm{COO}^{-}$

Clinopyroxene

$\mathrm{CO}_{2}$

$\mathrm{CO}$

$\mathrm{CO}_{2}$ Sequestration

Cradle-to-grave

Cradle-to-gate

Direct

EDTA

EIA

EIO-LCA

ELECNRTL

EOR
Åbo Akademi University

Ammonium sulphate, $\left(\mathrm{NH}_{4}\right)_{2} \mathrm{SO}_{4}$

Market leading chemical process simulation and optimization software

Unit of pressure, equivalent to $101325 \mathrm{~Pa}, 1.01325 \mathrm{bar}$

Processes on which no or, at best, indirect influence may be made by the decision maker for which a life cycle assessment study is carried out

Petroleum company, formerly known as British Petroleum

Bafokeng Rasimone Platinum MineJoint, South African based platinum mining company

Calcium, $\mathrm{Ca}^{2+}$ as calcium ions, $\mathrm{CaO}$ as calcium oxide

Calcium silicate, wollastonite

Carbon capture and storage

Chlorofluorocarbon

Methane, also natural gas

Acetic acid

Acetate ion

Monoclinic pyroxenes, either sodic or calcic, $(\mathrm{Na}, \mathrm{Al}, \mathrm{Ca}, \mathrm{Mg}, \mathrm{Fe})_{2} \mathrm{SiO}_{6}$

Carbon dioxide

Carbon monoxide

Long-term storage of carbon dioxide

Life cycle assessment from resource extraction to disposal

A partial product life cycle assessment, from resource extraction to through the production of the studied product, excluding use or end-oflife stages.

In reference to carbonation process routes, occurring in a single process step

ethylenediaminetetraacetic acid

Energy Information Administration

Economic input-output life cycle assessment

Electrolyte non-random two-liquid property method

Enhanced oil recovery 


\begin{tabular}{|c|c|}
\hline EPA & Environmental Protection Agency \\
\hline Ex-situ & Out of natural place, i.e., above ground \\
\hline $\mathrm{Fe}$ & Ferric iron, $\mathrm{Fe}^{2+}$ as iron ions, $\mathrm{FeO}$ as iron oxide \\
\hline $\mathrm{FeSiO}_{3}$ & Iron (II) silicate, Ferrosilite \\
\hline $\mathrm{Fe}(\mathrm{III})$ & Ferrous iron, $\mathrm{Fe}^{3+}$ as iron ions \\
\hline Foreground & $\begin{array}{l}\text { Processes which are under control by the decision maker for which a life } \\
\text { cycle assessment study is conducted }\end{array}$ \\
\hline Gate-to-gate & $\begin{array}{l}\text { A partial life cycle assessment focusing on only one value-added } \\
\text { process in the entire production chain }\end{array}$ \\
\hline GTL & Gas to liquids \\
\hline $\mathrm{GW} \cdot \mathrm{h}$ & Gigawatt hour \\
\hline GWP, GWP100 & Global warming potential, GWP over a 100 -yr time horizon \\
\hline $\mathrm{H}+$ & Hydrogen ion, proton \\
\hline $\mathrm{HCO}_{3}^{-}$ & Bicarbonate ion \\
\hline $\mathrm{HCOOH}$ & Formic acid \\
\hline $\mathrm{H}_{2} \mathrm{CO}_{3}$ & Carbonic acid \\
\hline $\mathrm{HFC}$ & Hydrofluorocarbon \\
\hline $\mathrm{HNO}_{3}$ & Nitric acid \\
\hline $\mathrm{H}_{3} \mathrm{PO}_{4}$ & Phosphoric acid \\
\hline $\mathrm{H}_{2} \mathrm{SO}_{4}$ & Sulphuric acid \\
\hline Heat integration & $\begin{array}{l}\text { A process engineering technique used to minimise overall process } \\
\text { energy consumption }\end{array}$ \\
\hline Heat-treatment & $\begin{array}{l}\text { Heating of a mineral to remove chemically bound water, e.g., } \\
\text { serpentine, also } H T\end{array}$ \\
\hline IEA & International Energy Agency \\
\hline Impala & South African based platinum mining company \\
\hline Indirect & $\begin{array}{l}\text { In reference to carbonation process routes, reactive components } \\
(\mathrm{Ca} / \mathrm{Mg}) \text { are first extracted from the mineral matrix prior to carbonation } \\
\text { in a separate step }\end{array}$ \\
\hline In-situ & In deposit \\
\hline IPCC & Intergovernmental Panel on Climate Change \\
\hline IPCC-AR5 & Intergovernmental Panel on Climate Change Assessment Report 5 \\
\hline ISO & International Organisation for Standards \\
\hline $\mathrm{kg}-\mathrm{CO}_{2} \mathrm{e}$ & kilograms of carbon dioxide equivalents \\
\hline
\end{tabular}


$\mathrm{KOH}$

KW

LANL

LCA

LCI

LCIA

Lonmin

$\mathrm{M}^{2+}$

MCT

$\mathrm{Mg}$

$\mathrm{MgCl}_{2}$

$\mathrm{MgCl}_{2} \cdot 6 \mathrm{H}_{2} \mathrm{O}$

$\mathrm{MgCl}(\mathrm{OH})$

$\mathrm{MgCO}_{3}$

$\mathrm{MgCO}_{3} \cdot 6 \mathrm{H}_{2} \mathrm{O}$

$\mathrm{Mg}\left(\mathrm{NO}_{3}\right)_{2}$

$\mathrm{Mg}(\mathrm{OH})_{2}$

$\mathrm{MgSiO}_{3}$

$\mathrm{Mg}_{2} \mathrm{SiO}_{4}$

$\mathrm{MgSO}_{4}$

Micron

$\mathrm{MJ} / \mathrm{hr}$

$\mathrm{M}(\mathrm{OH})_{2}$

Mt

MVR

MWe

$\mathrm{NaCl}$

$\mathrm{NaHCO}_{3}$

$\mathrm{NaOH}$

$\mathrm{NaSiO}_{3}$

NETL

NGCC
Potassium hydroxide

Kilowatt, $10^{3} \mathrm{~W}$

Los Alamos National Laboratory

Life cycle assessment

Life cycle inventory

Life cycle impact assessment

South African based platinum mining company

Metal ion

Mineral carbonation technologies

Magnesium, $\mathrm{Mg}^{2+}$ as magnesium ions, $\mathrm{MgO}$ as magnesium oxide

Magnesium chloride

Magnesium chloride hexahydrate

Magnesium chloride hydroxide

Magnesium carbonate

Hydromagnesite

Magnesium nitrate

Magnesium hydroxide

Magnesium silicate, enstatite, orthopyroxene

Olivine, forsterite

Magnesium sulphate

micrometer, $10^{-6} \mathrm{~m}, \mu \mathrm{m}$

Megajoules per hour

Metal hydroxide

Megatonne, $10^{9} \mathrm{~kg}$

Mechanical vapour recompression

Megawatt electric

Sodium chloride

Sodium bicarbonate

Sodium hydroxide

Sodium silicate

National Energy Technology Laboratory

Natural gas combined cycle 
$\mathrm{NH}_{3}$

$\mathrm{NH}_{4} \mathrm{Cl}$

$\mathrm{NH}_{4} \mathrm{HCO}_{3}$

$\mathrm{NH}_{4} \mathrm{HSO}_{4}$

$\mathrm{NH}_{4} \mathrm{NO}_{3}$

$\mathrm{NH}_{4} \mathrm{OH}$

Northam

$\mathrm{O}_{3}$

$\mathrm{OH}^{-}$

$\mathrm{P}_{\mathrm{CO}_{2}}$

PGM

Plagioclase

ppm

PTGA

PWC

Pyroxene

$\mathrm{R}_{\mathrm{CO}_{2}}$

RK-SOAVE

SACCS

SASOL

Serpentine

SimaPro

Si-O tetrahedral

$\mathrm{SiO}_{2}$

Stoichiometry

$\mathrm{t}-\mathrm{CO}_{2}$

Tailings

UNIQUAC

Weathering

$w t \%$
Ammonia

Ammonium chloride

Ammonium bicarbonate

Ammonium bisulphate

Ammonium nitrate

Ammonium hydroxide, also ammonia water

South African based platinum mining company

Ozone

Hydroxide ion

Carbon dioxide partial pressure

Platinum group metals

Al-Ca-rich silicate mineral, such as $\mathrm{NaAlSi}_{3} \mathrm{O}_{8}, \mathrm{CaAl}_{2} \mathrm{Si}_{2} \mathrm{O}_{8}$

Parts per million

Pressurized thermogravimetric analyser

PricewaterhouseCoopers company

Silicate mineral with general formula $(\mathrm{M} 1)(\mathrm{M} 2)(\mathrm{Si}, \mathrm{Al})_{2} \mathrm{O}_{6}$

A theoretical measure, defined as the mass of ore required to convert a unit mass of carbon dioxide into carbonate.

Redlich-Kwong-Soave equation of state model

South African Centre for Carbon Capture \& Storage

South African based international integrated chemicals and energy company

Mg-sich silicate mineral, chemical formula $\mathrm{Mg}_{3} \mathrm{Si}_{2} \mathrm{O}_{5}(\mathrm{OH})_{4}$

Widely used life cycle assessment software

$\mathrm{SiO}_{4}^{4-}$ molecule

Silicon dioxide

The ratio of elements to each other in a mineral

ton of carbon dioxide

The waste mineral material from the processing of ore

Universal quasi-chemical property method

The breakdown of rocks and minerals through contact with the earth's atmosphere over geological time-frames

weight percentage, also mass percentage 


\section{Chapter 1}

\section{Introduction}

The world's energy mix is heavily biased towards fossil fuels combustion to meet global demand, providing more than $80 \%$ of the world's primary energy supply (IEA, 2013). This is expected to continue well into the $21^{\text {st }}$ century (Maroto-Valer et al., 2005). The combustion of fossil fuels releases $\mathrm{CO}_{2}$ into the atmosphere. The levels of $\mathrm{CO}_{2}$ concentration in the atmosphere have increased from a pre-industrial value of $280 \mathrm{ppm}$ to over $400 \mathrm{ppm}$ in 2014 (Huisingh et al., 2015). Primarily due to anthropogenic activities, the Intergovernmental Panel on Climate Change (IPCC) has linked this increase to global climate change. This has raised concerns due to the potentially devastating effects of climate change that include rising sea levels and extreme weather patterns (IPCC-AR5, 2013). This has led some to describe global climate change as "one of the greatest threats to human survival in history" (Huisingh et al., 2015).

South Africa is a fossil fuel dependent economy with more than $95 \%$ of energy consumption coming from coal, natural gas and oil (British Petroleum, 2013). This has made South Africa the leading $\mathrm{CO}_{2}$ emitter in Africa, $14^{\text {th }}$ in the world (EIA, 2014), and the only country in Africa in the top 20 of global emitters. It is estimated that $440 \mathrm{Mt}$ of $\mathrm{CO}_{2}$ is released into the atmosphere per annum (Doucet, 2011).

The Government of South Africa has ratified the United Nations Convention on Climate Change and its Kyoto Protocol making commitments to address climate change. It identifies climate change as "one of the threats to sustainable development" that threaten to undermine development according to the Millennium Development Goals (Department of Environmental Affairs, 2011). This suggests that this is a matter of strategic importance for the country and requires serious attention to effectively address. In this regard, technological solutions that reduce or taper rising $\mathrm{CO}_{2}$ emissions need to be explored in order to prevent adverse effects on the environment, which would undermine the development objectives of South Africa.

The combustion of fossil fuels to power economies, particularly in the developing world, is unlikely to be eliminated in the near future. This is due to the vast availability of these resources, and the relatively well understood and less costly technologies to harness energy 
from these fuels. In view of this, improvements in energy efficiency, development of clean alternative energy sources, as well as carbon capture and storage (CCS) technologies are required to reduce $\mathrm{CO}_{2}$ emissions. Improvements to energy efficiency are limited (Wang, 2011), and thus would not contribute significantly to emissions reduction. With the adoption of alternative clean energy sources being very slow, the consideration of CCS technologies to provide a more immediate solution is necessary. This technology can reduce between $85-90 \%$ of the $\mathrm{CO}_{2}$ emissions from a large point source emitter (Leung et al., 2014). An overview of the carbon capture and storage process is presented in Figure 1-1.

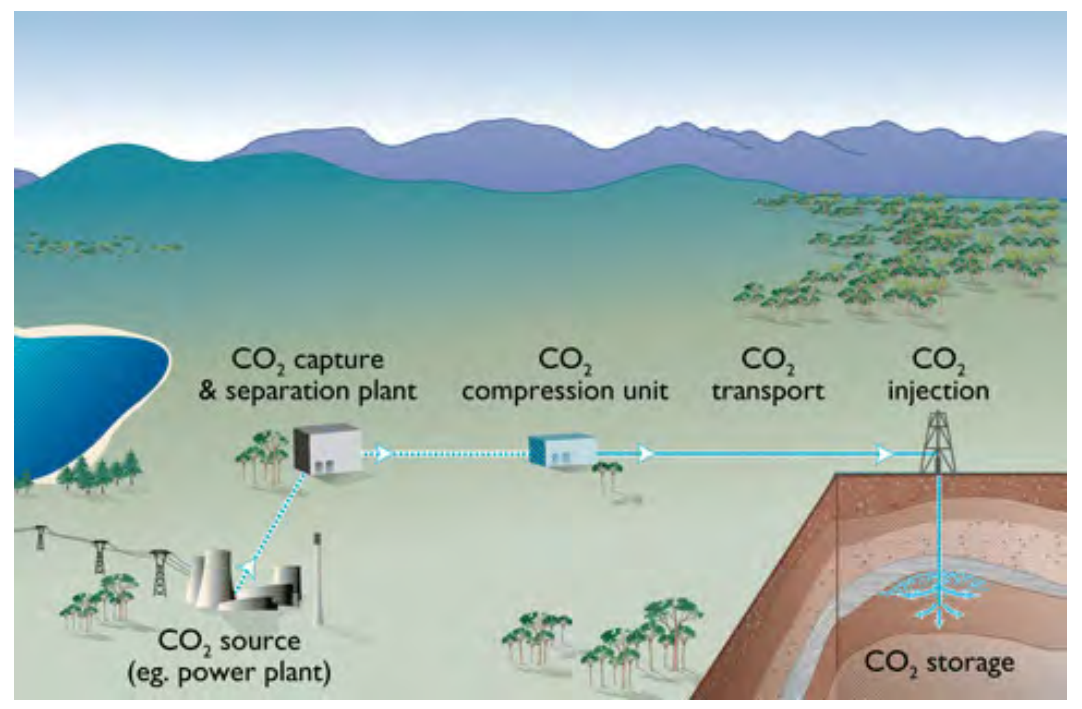

Figure 1-1: Carbon capture and storage overview schematic (CO2CRC, 2015)

The South African government has established the South African Centre for Carbon Capture $\&$ Storage (SACCS), with a view towards implementing these technologies in the country (SACCS, 2013). The IPCC (2005) states that CCS involves collection/concentration from emitters such as industrial and energy generating operations, transport to storage locations and storage away from the atmosphere for a long period of time. The storage of $\mathrm{CO}_{2}$ can be classed into three categories: ocean storage, geological storage and mineral carbonation. Despite the general concerns with potential leakages (Park, 2005; IPCC, 2005, Teir, 2008) associated with geological and ocean storage, limited sites for geological storage exist in South Africa, and offshore basins are far from $\mathrm{CO}_{2}$ emitters (Cloete, 2010; Viljoen et al., 2010). This suggests that implementing these technologies in South Africa may not be worthwhile, since the amounts sequestered may not be significant or the costs of sequestration too high to justify. A potential alternative to these in South Africa is mineral carbonation. It is this potential that will 
be explored in this study, with a specific focus on the potential use of PGM tailings as feedstock.

\subsection{Background}

Mineral carbonation is a capture and storage technology that mimics the natural weathering process through the reaction of oxides or silicates of magnesium, calcium or iron with $\mathrm{CO}_{2}$ to form stable mineral carbonates. The process is summarized by the chemical equation:

$$
(\mathrm{Ca}, \mathrm{Mg}, \mathrm{Fe}) \mathrm{SiO}_{3(\mathrm{~s})}+\mathrm{CO}_{2(\mathrm{~g})} \rightarrow(\mathrm{Ca}, \mathrm{Mg}, \mathrm{Fe}) \mathrm{CO}_{3(\mathrm{~s})}+\mathrm{SiO}_{2(\mathrm{~s})}
$$

The stability of the reaction products of mineral carbonation is a result of the carbon atom being at its lowest energy state in the carbonate product that provides storage on a time scale of $>100,000$ years (Rackley, 2010). The use of silicates in mineral carbonation is also attractive due to their abundance in nature, with a potential to sequester all $\mathrm{CO}_{2}$ emissions from the combustion of all existing fossil fuel resources (Lackner et al., 1995; IPCC, 2005). Finally, the mineral carbonation reaction is exothermic thus presenting energy integration opportunities in a developed industrial mineral carbonation process. A schematic of the mineral carbonation process is shown in Figure 1-2.

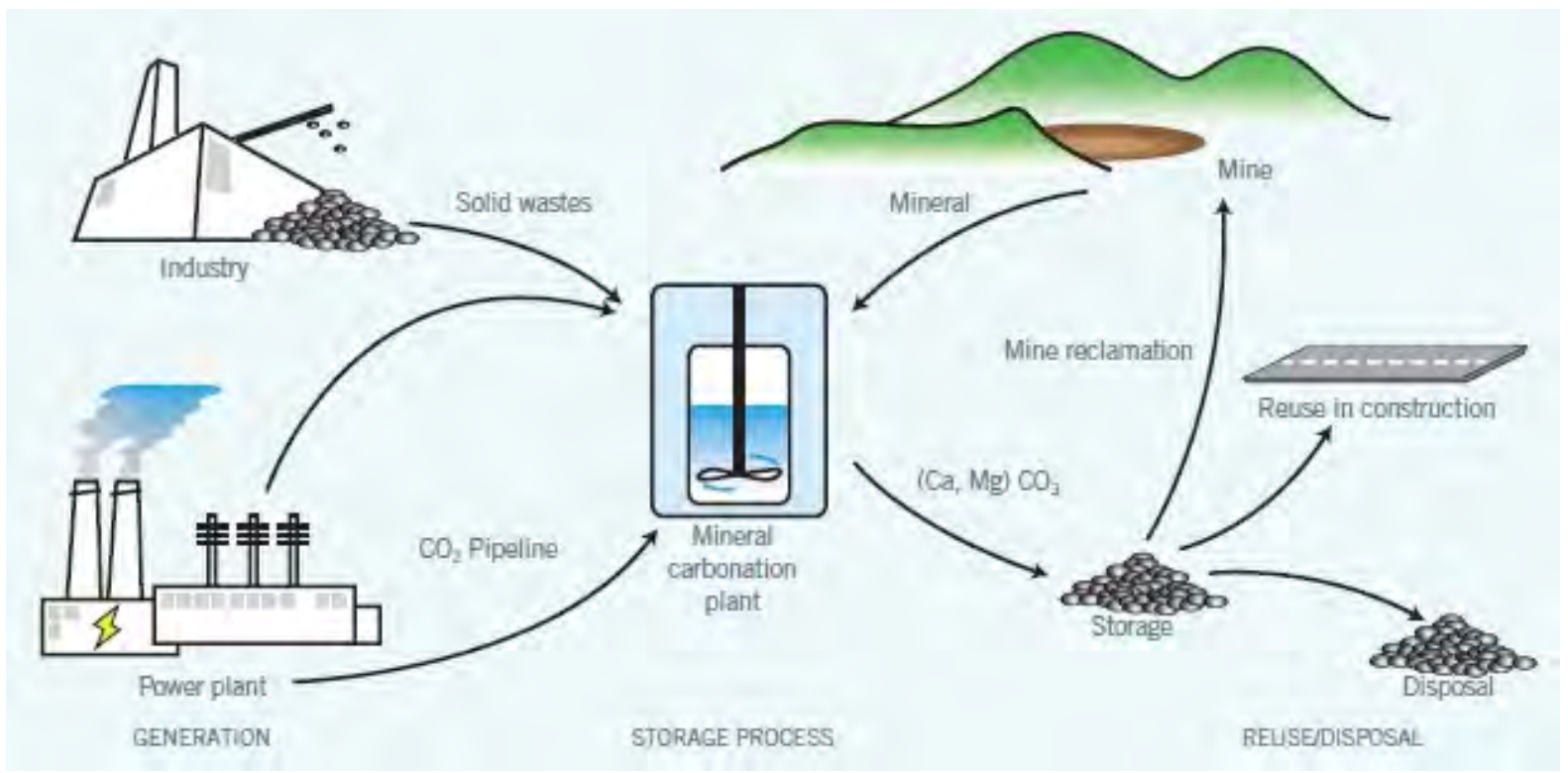

Figure 1-2: Mineral carbonation process scheme (Global CCS Institute, 2011)

\subsubsection{Engineered Mineral Carbonation Processes}

Since mineral carbonation mimics the natural weathering process, which occurs over geological time scales, the reaction kinetics for this process are inherently slow. Several approaches to mineral carbonation have been studied ranging from direct carbonation of solid 
mineral with $\mathrm{CO}_{2}$, to more complex multi-stage aqueous mineral carbonation routes (Rackley, 2010; Olajire, 2013). The primary focus of research in this area has been to accelerate the reaction kinetics of mineral carbonation so that it may be justified for industrial implementation. Figure 1-3 provides a summary of the mineral carbonation technologies (MCT) that have been proposed in literature. The mineral carbonation processes have been classified into ex-situ (out of natural place, i.e., above ground), in-situ (in deposit), as well as other routes that include the use of biological organisms to facilitate the carbonation process. It is evident that several process routes have been proposed within these categories. However, the subsequent discussion will focus on some of the more common ex-situ carbonation processes.

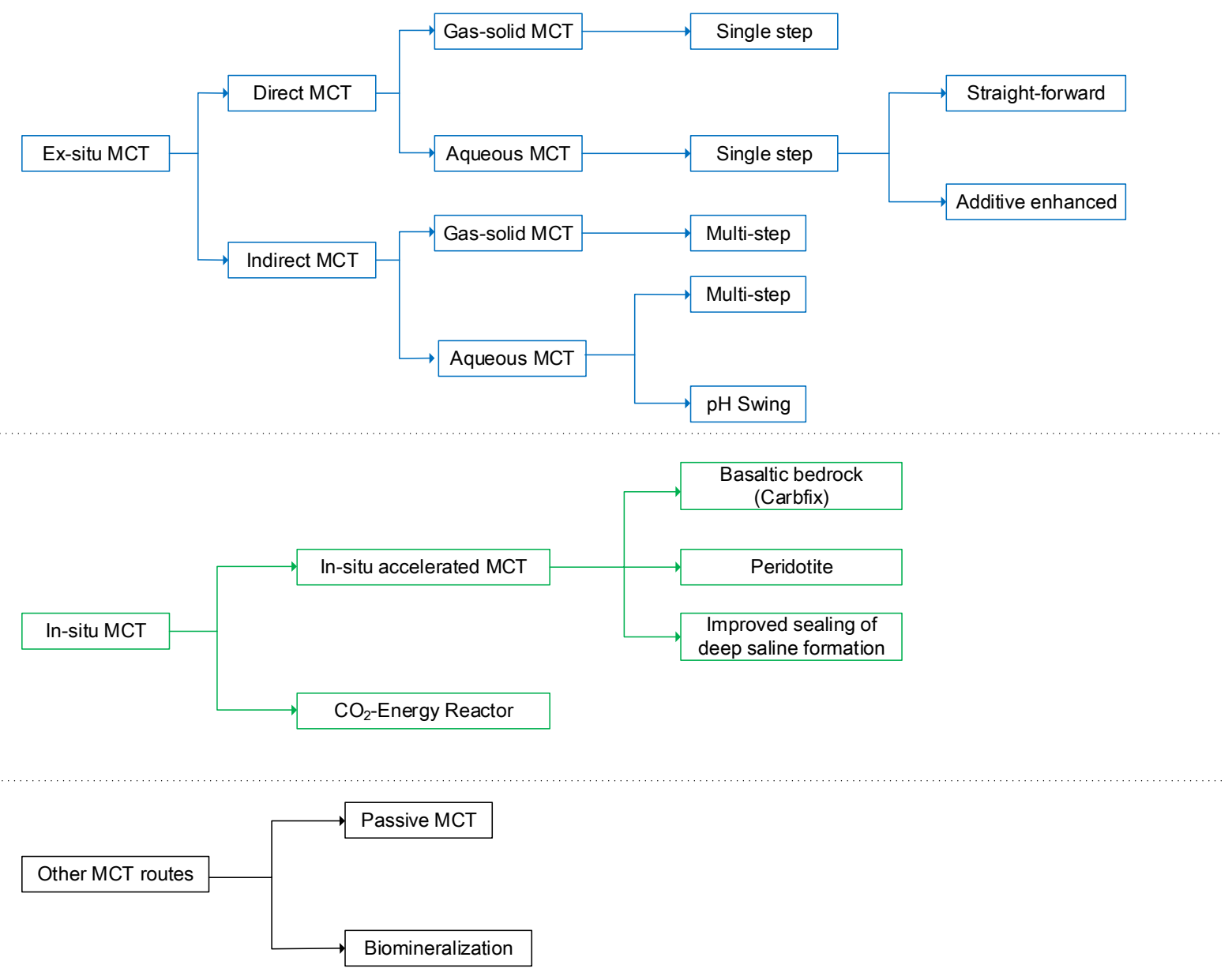

Figure 1-3: Classification of mineral carbonation process routes (adapted from Olajire, 2013)

The simplest mineral carbonation route, in terms of process design, is the direct gas/solid carbonation route. This approach is hindered by prohibitively slow reaction kinetics even at elevated temperatures and pressure (Huijgen and Comans, 2003). It has thus been virtually abandoned, though a few researchers (Fagerlund et al., 2010; Zevenhoven et al., 2011; Slotte 
et al., 2013) are still conducting research in this route, albeit having developed this into a relatively more complex indirect multi-stage process.

A considerable amount of research interest has been given to the aqueous mineral carbonation routes (Huijgen and Comans, 2003). This approach makes use of water or chemical additives to accelerate the mineral carbonation process. Direct aqueous mineral carbonation, in particular, has enjoyed intense interest by a number of researchers (O'Connor et al., 2001; Gerdemann et al., 2007; Bonfils et al., 2012) who consider it to be more promising than gassolid approaches. However, aqueous mineral carbonation involves two steps, which have conflicting optimal operating conditions: extraction of the alkaline earth metals from the silicate minerals through dissolution, followed by carbonation of the dissolved cations, through carbonate formation and precipitation. A low $\mathrm{pH}$ and high temperature are optimal for the dissolution step whereas the precipitation step is favoured by a high $\mathrm{pH}$ and more moderate temperatures (Gerdemann et al., 2007). This approach, by design, is thus limited in terms of process optimisation because of the complexity of the carbonation system (Haug, 2010).

Park et al. (2003) proposed a pH swing method, an indirect aqueous mineral carbonation approach. This approach involves dissolving silicate minerals at low $\mathrm{pH}$ using acid solutions and subsequently carbonating the leachate under alkaline conditions. This provides the opportunity to optimise the dissolution and carbonation reaction steps individually. It has thus been adopted by a significant number of researchers (Teir et al. 2007; Wenzhi et al., 2009; Wang and Maroto-Valer, 2011; Meyer et al., 2013; Sanna et al., 2014a). The dissolution stage is considered to be the rate-limiting step in aqueous mineral carbonation (Park and Fan., 2004; Alexander et al., 2007; Gerdemann et al., 2007; Meyer et al., 2014), and much of the research and development of engineered mineral carbonation systems has focused on methods to improve kinetics of the dissolution step.

To accelerate the dissolution kinetics, aggressive leach conditions (low $\mathrm{pH}$, elevated temperatures and pressures, strong acids) and/or pretreatment such as mechanical and thermal activation have been suggested (Gerdemann et al., 2007; Teir et al., 2007; Wang and MarotoValer, 2011). However, these conditions are relatively energy intensive (Maroto-Valer et al., 2005; Gerdemann et al., 2007), and can significantly increase the $\mathrm{CO}_{2}$ footprint of the mineral carbonation process as a result of the process energy requirements. It is important then that the sequestration benefits of the mineral carbonation solution, outweigh the environmental impact that it causes. 
This is an important consideration, since the fundamental purpose of mineral carbonation technology should be to reduce, not increase $\mathrm{CO}_{2}$ emissions. This means that for the mineral carbonation process to be a viable $\mathrm{CO}_{2}$ emissions reduction technology, the process needs to result in negative net $\mathrm{CO}_{2}$ emissions over its entire life cycle. This would include carbon capture and storage, transportation, materials preparation, carbonation, consumption and regeneration of reagents, and management of solid products and effluents.

\subsubsection{PGM Tailings as potential feedstock}

The precious metals industry in South Africa produces vast amounts of mine tailings that contain silicate minerals. In particular, the platinum industry produces 77.5 million tonnes per annum of PGM tailings that, in theory, can sequester 13.5 million tonnes of $\mathrm{CO}_{2}$ per annum (Vogeli et al., 2011). These are dominated by magnesium and calcium silicates, and are mostly orthopyroxene (65 wt.\%) and plagioclase (16 wt.\%) with clinopyroxene and amphibole in smaller amounts (Meyer et al., 2014). They are available as finely ground material (Vogeli et al., 2011) due to ultra-fine grinding required to liberate small platinum group grains. This implies that mechanical activation of this feedstock would not be required (Doucet, 2011). This indicates that there is potential for PGM tailings to be used as a mineral feedstock in mineral carbonation. An additional potential benefit to the use of this particular feedstock, is the opportunity to recover residual PGM mineral values during cation extraction in carbonation operations. This could potentially improve the economics of the process.

South Africa has a number of $\mathrm{CO}_{2}$ emission sites that are located within a $300 \mathrm{~km}$ radius of the PGM industry (Doucet, 2011). This makes it feasible to transport captured carbon dioxide to the tailings deposit for the purposes of mineral carbonation. Picot et al. (2011) established a world inventory which mapped carbon dioxide emissions sites to "Large and Superlarge Deposits" of silicate minerals, by linking these two data sources using ArcGIS technology. This global study identified the situation in South Africa as "favourable", in terms of proximity, as a number of $\mathrm{CO}_{2}$ emissions sites were within a $300 \mathrm{~km}$ radius from deposits of ultramafic rocks as indicated in Figure 1-4.

In particular, the Sasol Synfuels plants in Secunda (Mpumalanga) and Sasolburg (Free State) produce a highly pure stream of $\mathrm{CO}_{2}(90-98 \%)$ that would require minimal separations and $\mathrm{CO}_{2}$ capture operations (Cloete, 2006). This presents an attractive opportunity to develop a process that utilises this stream (largest global point-source polluter) and the mine tailings from the platinum industry, to create a uniquely South African carbon sequestration process. 


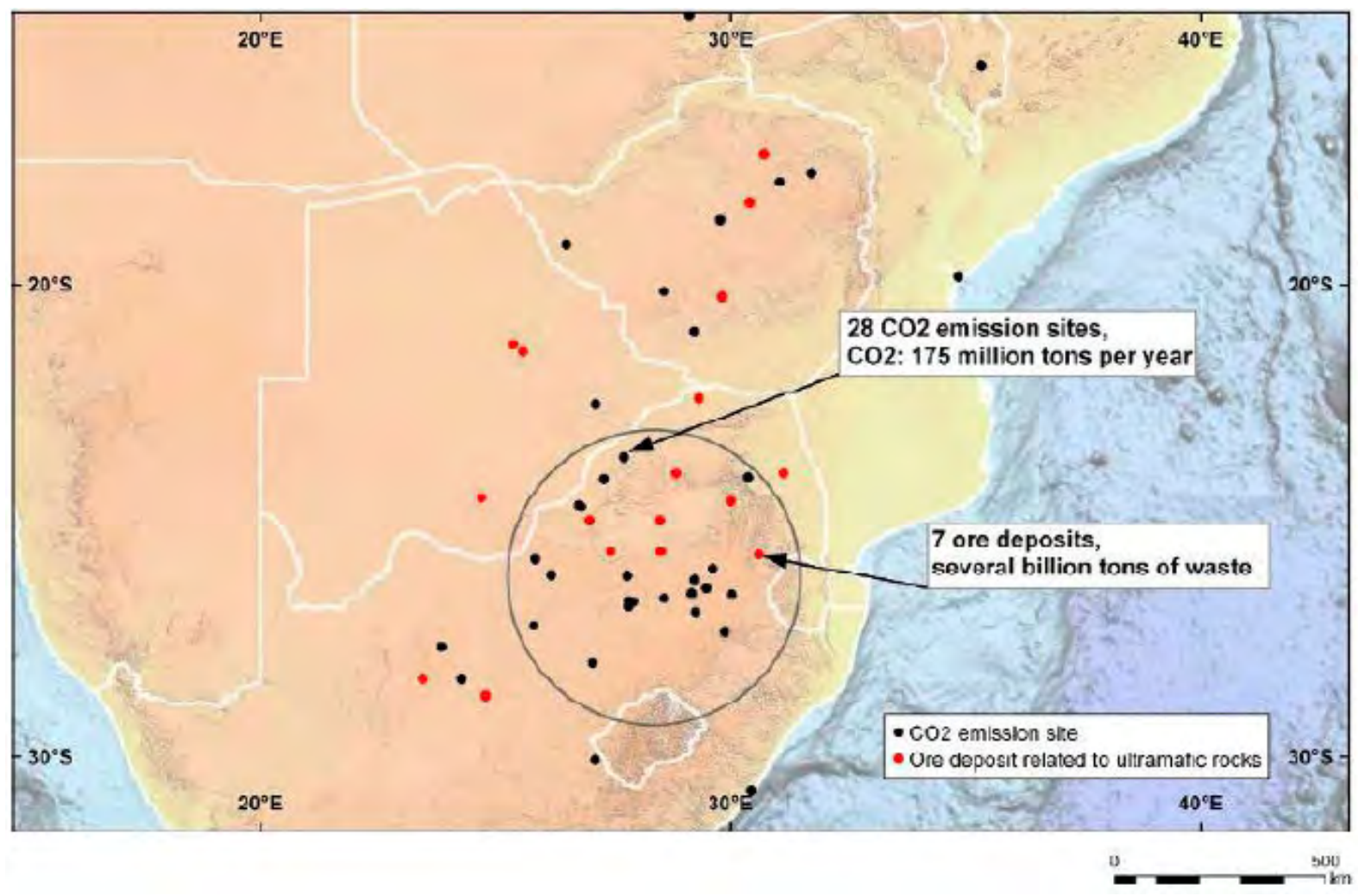

Figure 1-4: Carbon dioxide emissions sites and ultramafic rock deposits in $300 \mathrm{~km}$ radius (Picot et al., 2010)

However, preliminary experiments under relatively mild $\mathrm{pH}$ swing conditions have resulted in poor extraction efficiencies, which have been attributed to the relatively inert nature of the silicate mineral pyroxene (Meyer et al., 2014; Sanna et al., 2014a).

\subsection{Problem Statement}

South Africa is the continent's largest emitter of $\mathrm{CO}_{2}$, a greenhouse gas that has been linked to climate change. Limited geological sequestration sites are available to sequester the vast amounts of $\mathrm{CO}_{2}$ produced by industrial operations annually. Mineral carbonation is an attractive alternative that can potentially utilize the tailings produced by the platinum industry as feedstock. However, current carbonation of silicate minerals in engineered carbonation systems generally requires relatively aggressive and energy-intensive processes, and/or pretreatment to enhance the reaction kinetics. This is particularly so in the case of PGM tailings, the major component of which is pyroxene, which is very inert. This is a challenge given that a key requirement of any engineered mineral carbonation process, is that it must result in a net reduction in carbon dioxide emissions. Although a number of processes for the sequestration of carbon dioxide have been proposed, to date, rigorous and systematic studies on their carbon neutrality have been limited. 


\subsection{Research Objectives and Scope}

The overarching aim of this study is to investigate the viability of using PGM tailings to sequester $\mathrm{CO}_{2}$ on the basis of carbon neutrality. More specifically, this project sets out to meet the following objectives:

I. Identify potentially feasible flowsheets for the mineral carbonation of PGM tailings on the basis of literature data and information.

II. Develop mass and energy balances for the identified processes using process simulation software.

III. Establish the carbon footprint of the selected mineral carbonation processes using a lifecycle based approach.

This is a desktop study that makes use of published literature, and modelling assumptions based on literature and engineering heuristics to establish the carbon footprint of mineral carbonation processes. Experimental work was not conducted to verify or establish the technical credentials and feasibility of selected mineral carbonation processes. The identification of potentially feasible processes for the mineral carbonation of PGM tailings was based on a review of published literature and previous in-house (Vogeli et al., 2011; Vogeli, 2012; Meyer et al., 2014; Meyer, 2014) studies. The verification of the technical validity of the selected mineral carbonation processes is beyond the scope of this study. Additionally, the economic implications of the selected processes and process configurations was not evaluated.

\subsection{Dissertation Layout}

This dissertation consists of six chapters, including this introductory one. The introductory chapter (Chapter 1) gives an outline of the impact of carbon dioxide on the environment, and the viable storage options for carbon dioxide sequestration in South Africa, to provide a contextual background and motivation for the project. The mineral carbonation of PGM tailings is identified as potentially feasible, and a brief overview of process routes is provided. This is followed by a review of the relevant mineral carbonation related literature in Chapter 2, with specific focus on current mineral carbonation routes and engineered processes, their technical, and environmental performance. Additionally, a discussion on the selection of mineral carbonation processes to be considered is presented. As a consequence of the literature review, the methodology (Chapter 3) is developed that outlines the approach, tools and techniques used, including the definition of the goal and scope, material and energy balance evaluation, as well as inventory analysis and carbon emissions accounting. The results for selected processes are 
then presented and discussed individually in Chapter 4. In Chapter 5, a detailed comparison of the performance of selected processes is undertaken and discussed alongside some general discussions on the technical feasibility of selected processes. Final conclusions are then drawn and recommendations for further studies made in Chapter 6.

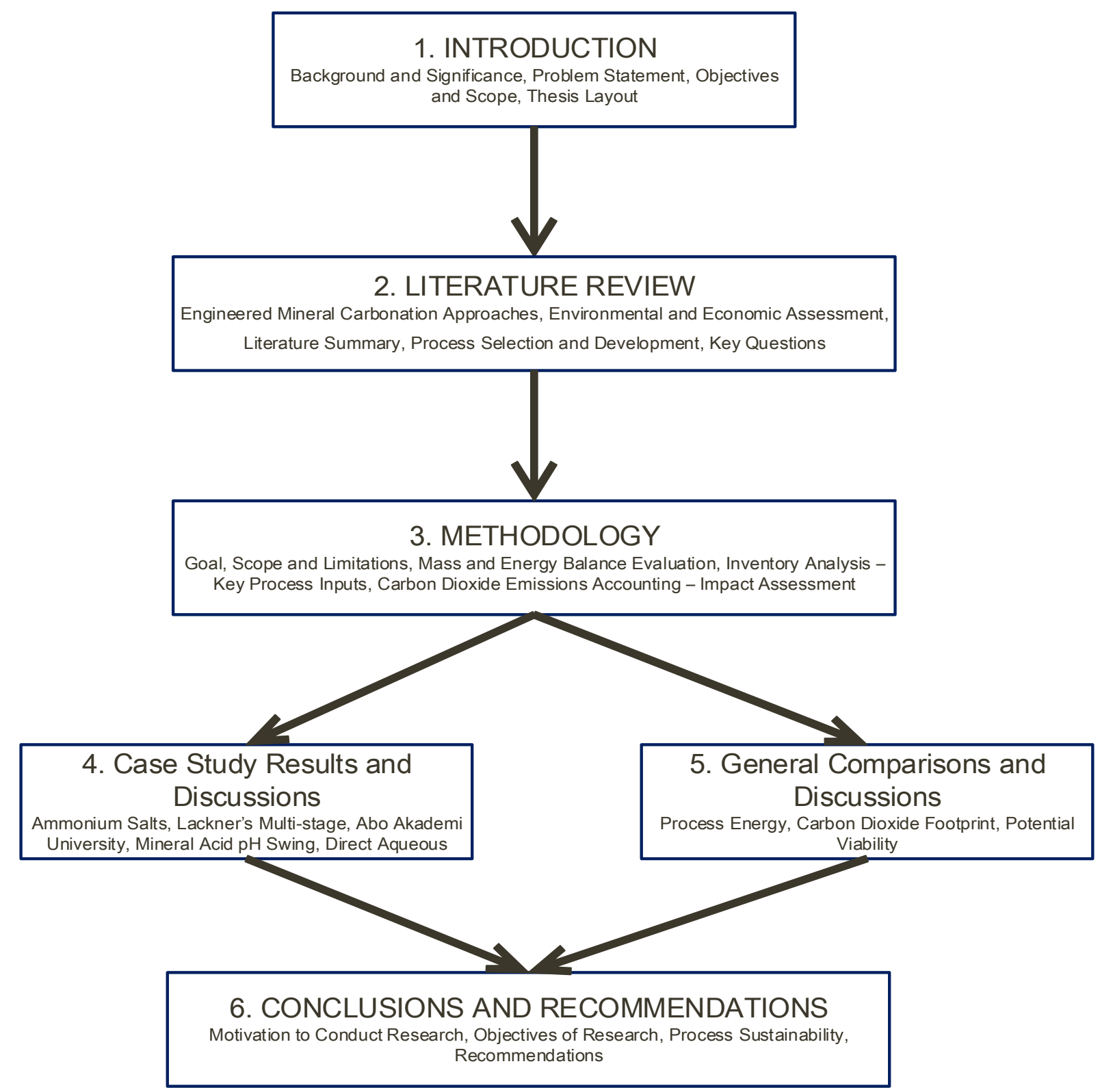

Figure 1-5: Outline of thesis layout 


\section{Chapter 2}

\section{Literature Review}

This literature review begins by reviewing the existing engineered mineral carbonation processes to gain insights into the state-of-the-art of these technologies. This assists in identifying potentially feasible process options, and in determining input data for mass and energy balance modelling. An exploration of previous life cycle assessment studies of carbonation processes is conducted to identify the key issues and the techniques used in evaluating process performance from an environmental standpoint.

\subsection{Engineered Mineral Carbonation Approaches}

The silicate minerals suitable for carbon sequestration through mineral carbonation are listed in Table 2-1 together with their theoretical sequestration capacity, $\mathrm{R}_{\mathrm{CO}_{2}}$. This is a theoretical measure defined as the mass of ore required to convert a unit mass of carbon dioxide into carbonate (Gerdemann et al., 2007). The silicate minerals with the lowest $\mathrm{R}_{\mathrm{CO}_{2}}$ values are olivine and serpentine.

Table 2-1: Direct carbonation based theoretical capacities of silicate minerals to sequester $\mathrm{CO}_{2}$ (Meyer et al., 2014)

\begin{tabular}{|c|c|c|c|c|c|c|c|c|}
\hline \multicolumn{2}{|l|}{ Mineral } & \multirow[t]{2}{*}{ Formula } & \multirow[t]{2}{*}{ Silicate Structure } & \multicolumn{3}{|c|}{ Ideal Elemental Concentrations (wt\%) } & \multirow[t]{2}{*}{$\mathrm{RCO}_{2}{ }^{\mathrm{a}}$} & \multirow[t]{2}{*}{ Rate $-\log \mathrm{R}\left(\mathrm{mol} \mathrm{cm}^{-2} \mathrm{~s}^{-1}\right)$} \\
\hline Group & End Member & & & $\mathrm{Ca}$ & $\mathrm{Fe}$ & $\mathrm{Mg}$ & & \\
\hline \multicolumn{9}{|l|}{ Feldspar } \\
\hline Plagioclase & Anorthite & $\mathrm{CaAl}_{2} \mathrm{Si}_{2} \mathrm{O}_{8}$ & Framework silicate & 10.3 & 3.1 & 4.8 & 4.4 & $9.3-14.5$ \\
\hline \multicolumn{9}{|l|}{ Pyroxene } \\
\hline Orthopyroxene & Enstatite & $\mathrm{MgSiO}_{3}$ & Inosilicate & 0.3 & 4 & 20.8 & 2.4 & $13.9-14.5$ \\
\hline \multirow[t]{2}{*}{ Clinopyroxene } & Diopside & $\mathrm{CaMgSi}_{2} \mathrm{O}_{6}$ & Inosilicate & 18.5 & 0.5 & 11.3 & 2.4 & $14-12$ \\
\hline & Augite & $\mathrm{CaMgSi}_{2} \mathrm{O}_{6}+(\mathrm{Fe}, \mathrm{Al})$ & Inosilicate & 15.6 & 9.6 & 6.9 & 2.7 & 13.5 \\
\hline Pyroxenoid & Wollastonite & $\mathrm{CaSiO}_{3}$ & Inosilicate & 31.6 & 0.5 & 0.3 & 2.8 & 12.4 \\
\hline \multicolumn{9}{|l|}{ Olivine } \\
\hline \multirow[t]{2}{*}{ Olivine } & Forsterite & $\mathrm{Mg}_{2} \mathrm{SiO}_{4}$ & Neosilicate & 0.1 & 6.1 & 27.9 & 1.8 & 12 \\
\hline & Fayalite & $\mathrm{Fe}_{2} \mathrm{SiO}_{4}$ & Neosilicate & 0.6 & 44.3 & 0.3 & 2.8 & 10.8 \\
\hline \multicolumn{9}{|l|}{ Serpentine } \\
\hline Serpentine & Antigorite & $\mathrm{Mg}_{3} \mathrm{Si}_{2} \mathrm{O}_{5}(\mathrm{OH})_{4}$ & Phyllosilicate & $<0.1$ & 2.4 & 24.6 & 2.1 & - \\
\hline
\end{tabular}

${ }^{a}$ Mass ratio of ore necessary to carbonate unit mass of $\mathrm{CO}_{2}$ 
Apart from their theoretical sequestration capacity, these silicates also differ in their structures and reactivity. Meyer et al. (2014) ranks the reactivity of magnesium silicates as decreasing in the order: olivine $>$ serpentine $>$ pyroxene. The reactivity of silicate minerals has been attributed to their structure. The structures of the ortho- and phyllo- silicates olivine and serpentine are less rigid, and held together by weaker bonds in comparison to pyroxene group minerals, that are an infinite, single chain of silica tetrahedral. This makes olivine and serpentine more reactive, as the cations are more accessible to the reagent (Meyer et al., 2014).

The natural weathering of these minerals, from which mineral carbonation is adapted, occurs over geological time scales. Consequently, the reaction kinetics of the mineral carbonation process would be very slow for industrial implementation under natural conditions. Engineered carbonation processes have thus been proposed and developed to accelerate the natural weathering process through the chemical and physical manipulation of the silicate mineral. This has included the use of high temperatures, high pressures, chemical reagents, and in some cases multiple process steps. This section will discuss the existing mineral carbonation process routes, their development, as well as the impact of changing process parameters on these routes.

\subsubsection{Gas-Solid Mineral Carbonation}

Direct gas-solid carbonation is considered the simplest mineral carbonation approach in terms of process design. This route also offers the best prospect of effective utilization of the heat released from the reaction (Rackley, 2010). This process was one of the first proposed for the purposes of mineral carbonation by researchers at the Los Alamos National Laboratory (LANL) (Lackner et al., 1995).

The main drawback of this process is that the carbonation reaction proceeds very slowly, and requires supercritical operating conditions $\left(300{ }^{\circ} \mathrm{C}\right.$ and 340 bar $\left.\mathrm{P}_{\mathrm{CO}_{2}}\right)$ to obtain reasonable reaction rates (Huijgen, 2007). A study conducted by Zevenhoven and Kohlmann (2002) found no detectable carbonation when the temperature was held constant at $200{ }^{\circ} \mathrm{C}$ in a pressurized thermogravimetric analyser (PTGA). Low conversions were reported even after samples were heated up to $1000{ }^{\circ} \mathrm{C}$ to release $\mathrm{MgO}$ from the silicate mineral. Secondly, increasing the temperature is thermodynamically limited since high temperatures favour gaseous $\mathrm{CO}_{2}$ instead of the carbonate compound due to entropy effects (Huijgen, 2007). This direct approach has been largely abandoned by most researchers (Olajire, 2013).

Studies then progressed towards a two-step route converting the silicate mineral to magnesium oxide at high temperatures and pressure to accelerate carbonation (Lackner et al., 1995; 
Zevenhoven et al., 2002). Zevenhoven et al. (2002) defines this process as occurring according to the reactions in equations (2) and (3):

$$
\begin{gathered}
\mathrm{xMgO} \cdot \mathrm{ySiO}_{2} \cdot \mathrm{zH}_{2} \mathrm{O}_{(\mathrm{s})} \rightarrow \mathrm{xMgO}_{(\mathrm{s})}+\mathrm{ySiO}_{2(\mathrm{~s})}+\mathrm{zH}_{2} \mathrm{O} \\
\mathrm{MgO}_{(\mathrm{s})}+\mathrm{CO}_{2} \rightarrow \mathrm{MgCO}_{3(\mathrm{~s})}
\end{gathered}
$$

The rate of carbonation of magnesium oxide under atmospheric conditions was reported to be too slow for practical purposes, whereas that of magnesium hydroxide was identified as promising (Lackner et al., 1997). This led these researchers to develop a processing scheme based on magnesium hydroxide. Studies conducted by Teir et al. (2006) demonstrated the impact of increasing temperature and pressure on the conversion of magnesium hydroxide to magnesium carbonate. Their findings showed that increasing the temperature and pressure for the carbonation of magnesium hydroxide increased the extent of carbonation. Table 2-2 indicates that the conversion in 6 hours did not exceed $60 \%$ even with temperatures exceeding $500{ }^{\circ} \mathrm{C}$ and a pressure of $45 \mathrm{bar}$, using commercially produced magnesium hydroxide. This is a serious limitation since substantial energy is required to achieve these conditions, with a modest return in terms of conversion.

Table 2-2: Conversion of $\mathrm{Mg}(\mathrm{OH})_{2}$ to $\mathrm{MgCO}_{3}$ at various temperature/pressure conditions after 6 hrs (Teir et al., 2006)

\begin{tabular}{|c|c|c|}
\hline Pressure bar & Temperature $^{\circ} \mathbf{C}$ & Conversion $\%$ \\
\hline 1 & 370 & 6 \\
\hline 12 & 460 & 19 \\
\hline 35 & 495 & 50 \\
\hline 35 & 510 & 46 \\
\hline 35 & 525 & 44 \\
\hline 35 & 540 & 43 \\
\hline 40 & 510 & 60 \\
\hline 40 & 525 & 37 \\
\hline 40 & 540 & 40 \\
\hline 45 & 525 & 44 \\
\hline
\end{tabular}

The magnesite produced by the carbonation reaction builds up on the surface of the silicate mineral particles creating a diffusion barrier that results in the slowing of the carbonation of magnesium hydroxide (Zevenhoven and Teir, 2004). Through the use of a fluidized bed 
reactor, Teir et al. (2004) demonstrated that this layer could be removed through attrition and abrasion.

Through further developments of this work, researchers at the Åbo Akademi University ( $\AA A U)$ have proposed a process that makes use of ammonium sulphate (AS) to convert the silicate mineral to magnesium hydroxide, through a high temperature and pressure solid-solid extraction reaction (Fagerlund et al., 2009). This reaction initially produces magnesium sulphate which is dissolved in water and subsequently reacted with ammonium hydroxide to precipitate, first impurities, then eventually the reactive magnesium hydroxide compound. This compound is finally reacted with gaseous carbon dioxide to produce solid and stable magnesite. A simple block-flow diagram demonstrating the main stages of the proposed process is presented in Figure 2-1.

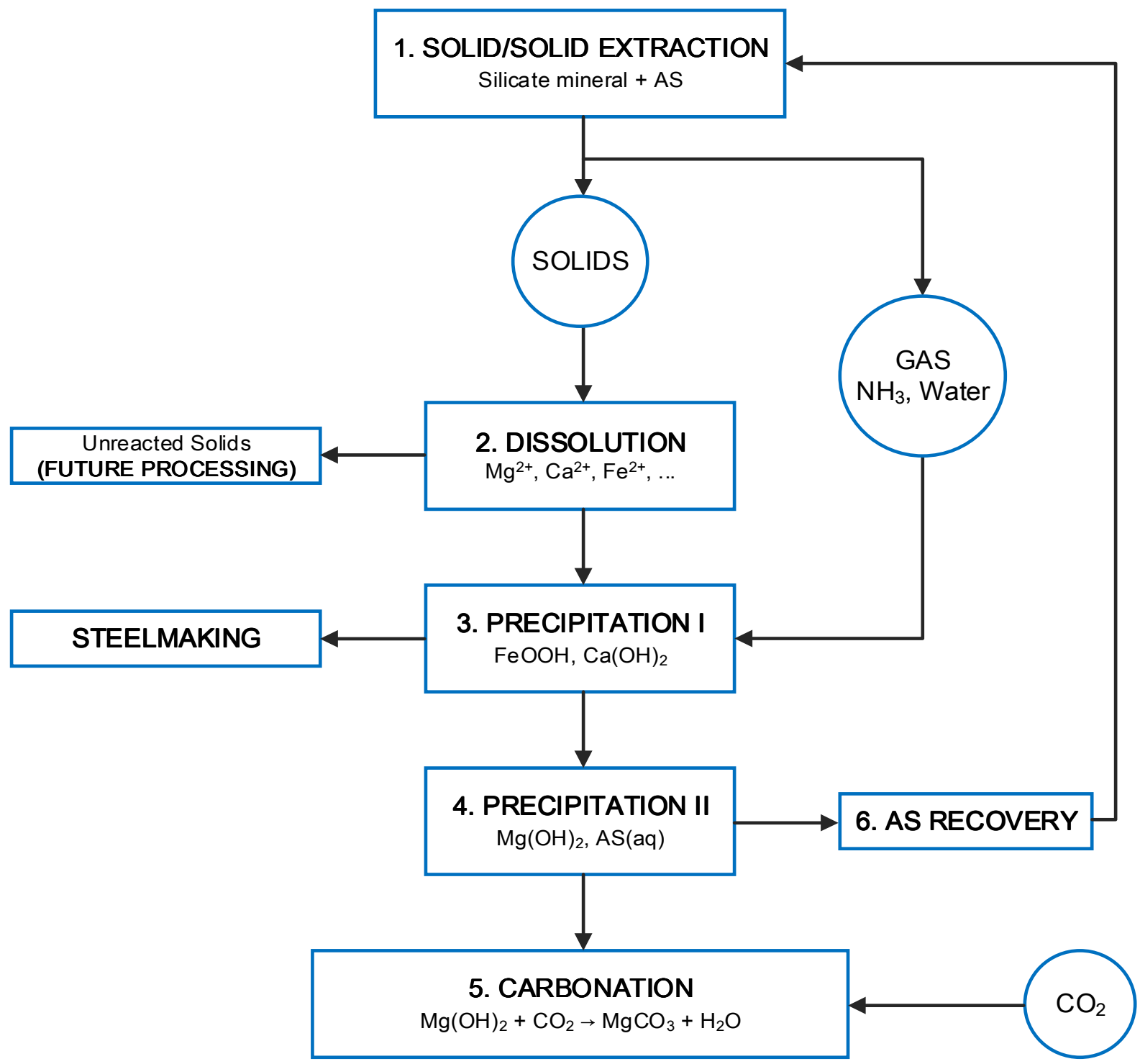

Figure 2-1: Schematic of Åbo Akademi University multi-stage process (adapted from Romao et al., 2012) 
The first step in the process proposed by researchers at $\AA \mathrm{AU}$ is the extraction of magnesium from the silicate mineral using ammonium sulphate. Nduagu et al. (2012a) reported that the maximum extraction obtained in his work was $64 \%-66 \%$ at $400-440{ }^{\circ} \mathrm{C}$ using a sample of serpentine with a particle size of 75-125 microns. This produces solid magnesium sulphate, and gaseous ammonia and water. A slightly lower (53\%) magnesium extraction was reported when the particle size fraction of 125-250 microns was used at about the same temperatures (Nduagu, 2012). The magnesium sulphate is then dissolved into solution and through a series of precipitation steps, is converted to magnesium hydroxide through reaction with water and ammonia, which also regenerates the ammonium sulphate reagent. This is followed by carbonation, which initial experimental data showed that the highest degree of conversion in the carbonation stage was $23 \%$, obtained by using $\mathrm{Mg}(\mathrm{OH})_{2}$ generated from serpentinite (Fagerlund et al., 2010). These experiments were conducted in a fluidized bed system that used high pressures $(2.85 \mathrm{MPa})$ and temperatures $\left(500{ }^{\circ} \mathrm{C}\right)$. A synthetic sample of $\mathrm{Mg}(\mathrm{OH})_{2}$ was used as feedstock, in another set of experiments. The authors reported that the process only managed $50 \% \mathrm{Mg}(\mathrm{OH})_{2}$ carbonation using this feedstock (Zevenhoven et al., 2011).

\subsubsection{Direct Aqueous Mineral Carbonation}

This is an approach that has garnered much research interest because of its better reaction kinetics in comparison to the gas-solid route. It is considered to be relatively simple since the two reaction steps in aqueous carbonation, dissolution and carbonation occur in a single step.

This process is based on the observation that natural weathering is enhanced by the presence of water. A process was developed by O'Connor et al. (2000) based on this phenomenon. In this process, $\mathrm{CO}_{2}$ at high pressure reacts in an aqueous suspension of the silicate mineral. The authors theorized that $\mathrm{CO}_{2}$ is dissolved in water to produce carbonic acid, which facilitates the extraction of the reactive cation $\left(\mathrm{Mg}^{2+}, \mathrm{Ca}^{2+}\right)$ from the mineral matrix. The free cation then reacts with bicarbonate ions generated by the dissociation of carbonic acid to produce a solid carbonate compound (O' Connor et al., 2000).

The process route thus involves three reaction steps that occur in a single reactor (Rackley, 2010):

Step 1: Carbon dioxide dissolves in water to form carbonic acid that dissociates to produce protons $\left(\mathrm{H}^{+}\right)$and bicarbonate ions $\left(\mathrm{HCO}_{3}^{-}\right)$, resulting in mildly acidic conditions:

$$
\mathrm{CO}_{2(\mathrm{~g})}+\mathrm{H}_{2} \mathrm{O}_{(\mathrm{l})} \rightarrow \mathrm{H}_{2} \mathrm{CO}_{3(\mathrm{aq})} \rightarrow \mathrm{HCO}_{3(\mathrm{aq})}^{-}+\mathrm{H}^{+}(\mathrm{aq})
$$


Step 2: The $\mathrm{H}^{+}$free protons extract $\mathrm{Ca} / \mathrm{Mg} / \mathrm{Fe}$ cations from the mineral matrix, e.g. for forsterite:

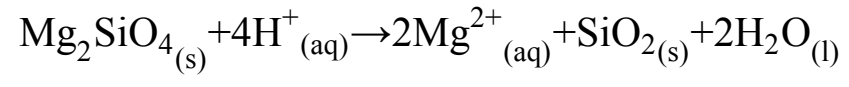

Step 3: The metal and bicarbonate ions combine and form carbonate precipitate:

$$
\mathrm{Mg}_{(\mathrm{aq})}^{2+}+\mathrm{HCO}_{3(\mathrm{aq})}^{-} \rightarrow \mathrm{MgCO}_{3(\mathrm{~s})}+\mathrm{H}_{(\mathrm{aq})}^{+}
$$

A large body of work has been done on this route that seeks to maximize the reaction rate and optimize the process (O'Connor et al., 2000; O'Connor et al., 2001, Gerdeman et al., 2002; O'Connor et al., 2005; Gerdemann et al., 2007). In developing the process, the first step would be to maximise the reaction rate by modifying the process conditions such as particle size, operating temperature, $\mathrm{CO}_{2}$ pressure and chemical additives.

\section{Impact of Temperature and Pressure}

The increase in temperature from ambient conditions generally increases the reaction rate of the direct aqueous carbonation process. On the other hand, this reduces the solubility of carbon dioxide in solution and makes the carbonation reaction less thermodynamically favourable (Gerdemann et al., 2002). This suggests that there are limits to the extent to which the temperature can be increased without significantly affecting overall carbonation through reducing carbon dioxide solubility. This, in turn, would indicate that there are optimal conditions which can be found that balance reaction rate with thermodynamic stability of the carbonate and $\mathrm{CO}_{2}$ solubility.

In work conducted by Gerdemann et al. (2002) at the Albany Research Center, relatively low temperatures less than $50{ }^{\circ} \mathrm{C}$ were found to favour the formation of hydromagnesite precipitates. Temperatures in the range between $100{ }^{\circ} \mathrm{C}$ and $150{ }^{\circ} \mathrm{C}$ were found to be the area of stability of magnesite, for $\mathrm{CO}_{2}$ pressure of 1 bar as well as 150 bar as indicated in Figure 2-2. The region of magnesium carbonate stability can be increased by increasing the pressure above atmospheric (Gerdemann et al., 2002). The increase in pressure also enhances the reaction rate due to the increased concentration of dissolved $\mathrm{CO}_{2}$. This is also because the reaction is pushed towards completion due to volume change as a result of gas consumption as the $\mathrm{CO}_{2}$ is converted to the carbonate compound (Gerdemann et al., 2002). 


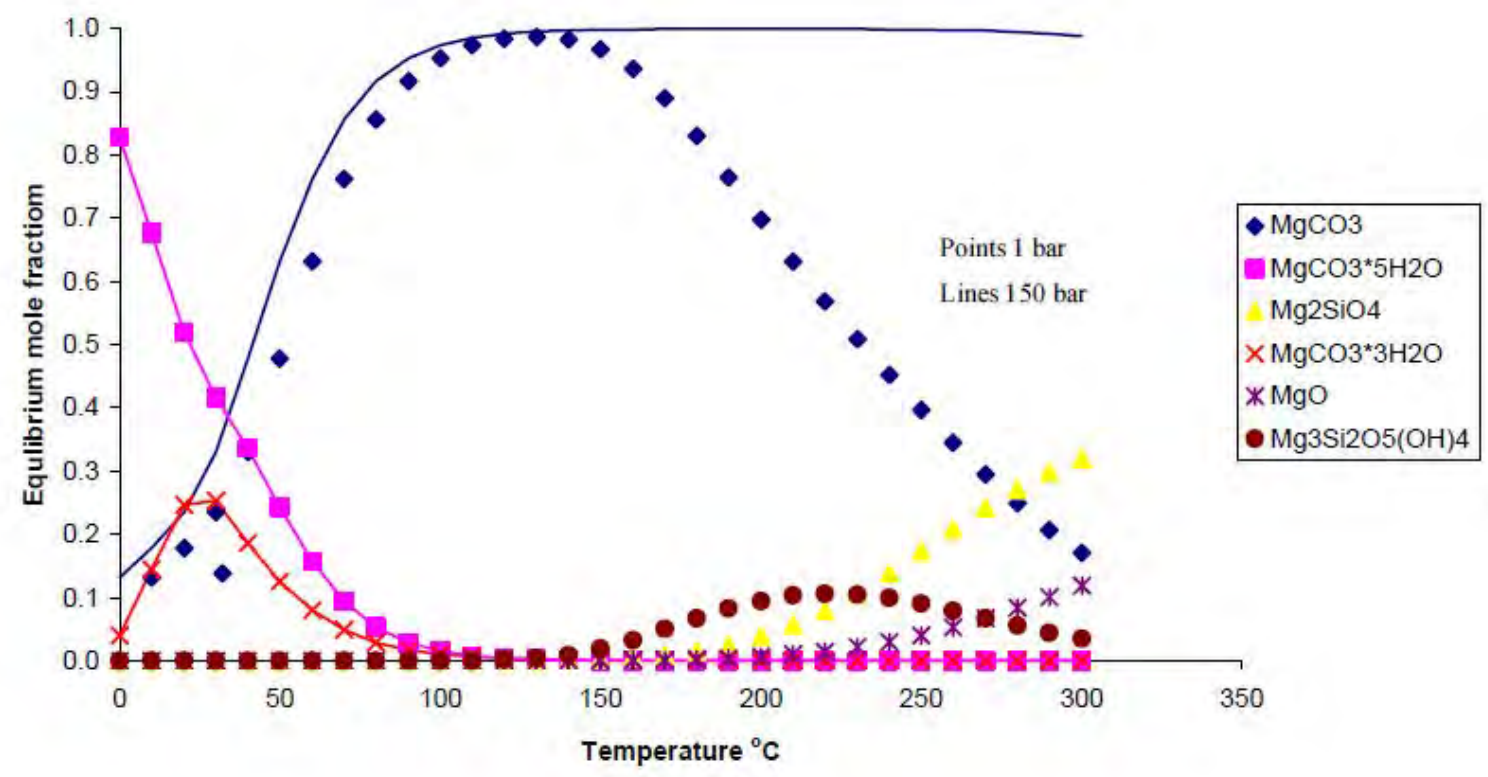

Figure 2-2: Thermodynamic equilibrium compositions for $\mathrm{Mg}_{2} \mathrm{SiO}_{4}-\mathrm{CO}_{2}-\mathrm{H}_{2} \mathrm{O}$ system (Gerdemann et al., 2002)

Studies on olivine conducted by the authors to establish the effect of pressure on extent of carbonation, demonstrated this increase in carbonation from atmospheric up to 250 atm (Gerdemann et al., 2002) as presented in Figure 2-3.

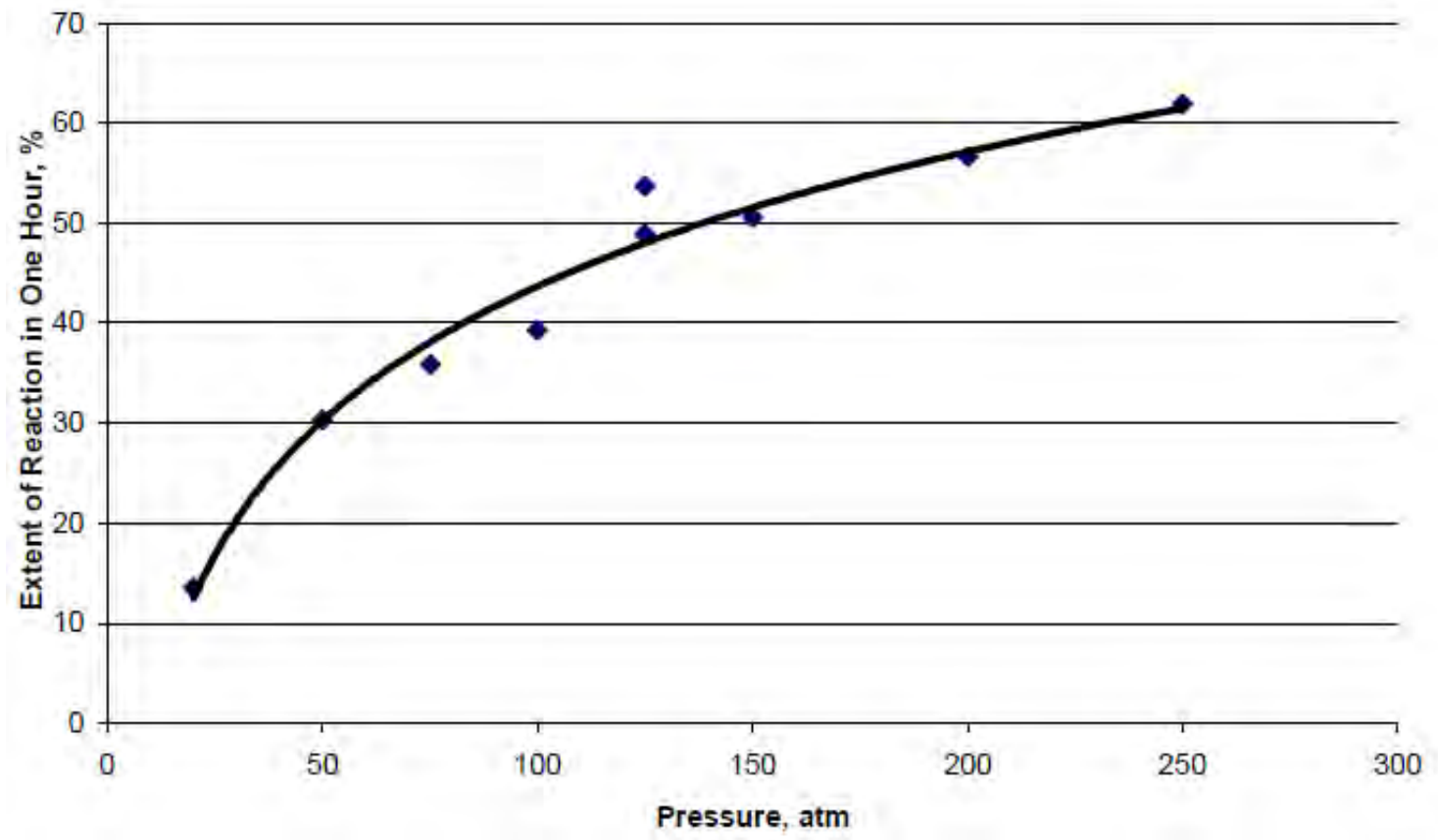

Figure 2-3: The effect of pressure on the extent of carbonation (Gerdemann et al., 2002)

Through the research from the Albany Research Center (now called the National Energy Technology Laboratory - NETL) optimum temperature, pressure and solution chemistry 
conditions have been proposed as indicated in Table 2-3. These high pressure and temperature conditions have been cited as energy intensive (Huijgen and Comans, 2003).

\section{Impact of Solution Chemistry}

The initial studies in direct aqueous mineral carbonation made use of distilled water as the $\mathrm{CO}_{2}$ carrying aqueous component (O'Connor et al., 2000). Work conducted at the Albany Research Center by O'Connor et al. (2002) showed that the use of sodium bicarbonate $\left(\mathrm{NaHCO}_{3}\right)$ and sodium chloride $(\mathrm{NaCl})$ significantly improved the reaction rate of the carbonation reaction. They postulated that the presence of sodium bicarbonate buffered the $\mathrm{pH}$ of the solution at $\sim 7.9$, and also provided carbonate ions that react with the silicate mineral to produce magnesite. The sodium chloride was believed to facilitate the formation of chloride complexes with $\mathrm{Mg}^{2+}$ cations. This reduced the concentration of the cations in solution, thus aiding further dissolution of the silicate mineral (O'Connor et al., 2000; O'Connor et al., 2005; Bodenan et al., 2014). As a result, the conversion of serpentine increased from $57 \%$ in 24 hours in distilled water to $82 \%$ in $0.64 \mathrm{M} \mathrm{NaHCO}_{3}$ and $1 \mathrm{M} \mathrm{NaCl}$ solution.

In a comprehensive study, adopting the methods developed by O'Connor et al. (2000), Gerdemann et al. (2007) used distilled water and $\mathrm{NaCl} / \mathrm{NaHCO}_{3}$ solutions for the mineral carbonation of olivine, wollastonite and serpentine in a direct aqueous mineral carbonation process. Their findings confirmed the observations of O'Connor et al. (2000), although they reported that carbonation of the Ca-silicate mineral, wollastonite, was independent of the solution used, as similar results were obtained for distilled water and $\mathrm{NaCl} / \mathrm{NaHCO}_{3}$ solutions. However, solution chemistry was found to have an effect on olivine and serpentine carbonation. The maximum carbonation of olivine $(>80 \%)$ and serpentine $(\sim 65 \%)$ was attained when $\mathrm{NaCl} / \mathrm{NaHCO}_{3}$ solution was used. The results of these studies are summarised in Table 2-3.

Table 2-3: Optimum carbonation conditions, by mineral (Adapted from Gerdemann et al., 2007)

\section{carbonation conditions}

\begin{tabular}{lccl}
\cline { 2 - 3 } \multicolumn{1}{c}{ Mineral } & $\mathbf{T},{ }^{\circ} \mathbf{C}$ & $\mathbf{P}_{\mathbf{C O} 2}, \mathbf{a t m}$ & \multicolumn{1}{c}{ carrier solution } \\
olivine & 185 & 150 & $0.64 \mathrm{M} \mathrm{NaHCO}_{3}, 1 \mathrm{M} \mathrm{NaCl}$ \\
wollastonite & 100 & 40 & distilled water \\
HT serpentine & 155 & 115 & $0.64 \mathrm{M} \mathrm{NaHCO}_{3}, 1 \mathrm{M} \mathrm{NaCl}$
\end{tabular}


Though the extents of carbonation attained in this study were relatively high, it important to note that these results were obtained under high temperatures $\left(150^{\circ} \mathrm{C}\right)$ and pressure $(150 \mathrm{~atm}$ $\mathrm{P}_{\mathrm{CO}_{2}}$ ). These conditions may result in severe energy penalties if adopted in industrial mineral carbonation processes.

The use of sodium salts that enhance the dissolution reaction has also been explored by researchers, in this process scheme, and improvements of up to $100 \%$ dissolution of magnesium in serpentine in $0.1 \mathrm{M}$ solutions of sodium citrate, sodium EDTA and sodium oxalate have been reported (Krevor and Lackner, 2009). Though this could be an option in terms of the dissolution of serpentine-based feedstocks, the impact of these reagents on the carbonation reaction is unclear, since carbonation is favoured by alkaline conditions.

\section{Impact of Mineral Type and Particle Size}

The results of the study by Gerdemann et al. (2007), summarised in Table 2-3 above, have indicated that optimal conditions are very mineral specific, with carbonation of wollastonite being optimum at much lower temperatures and pressures, and without reagent use in comparison to olivine and heat treated serpentine. Figure $2-4$ shows that more than $85 \%$ of olivine was converted to carbonate in a direct aqueous mineral carbonation process carried out at $185^{\circ} \mathrm{C}$ and $150 \mathrm{~atm} \mathrm{CO}_{2}$ using $\mathrm{NaCl} / \mathrm{NaHCO}_{3}$ solution. In comparison, serpentine reactivity was very low, and only improved to just over $60 \%$ with heat treatment of the serpentine samples, to remove chemically bound water (Gerdemann et al., 2007).

A decrease in particle size has been demonstrated to improve the reactivity of the silicate mineral for direct aqueous carbonation (O'Connor et al., 2001; Gerdemann et al., 2002). Studies conducted by O'Connor et al. (2001) indicated a substantial increase in carbonation with a reduction of particle size from minus 75 microns to minus 35 microns.

Reducing the particle size increases the surface area of silicate mineral available for reaction, in turn increasing the reaction rate (Gerdemann et al., 2007). However, particle size reduction can incur considerable energy penalties, with Gerdemann et al. (2002) reporting an energy penalty of about $230-240 \mathrm{KW} /$ ton of mineral for size reduction below 75 microns. 


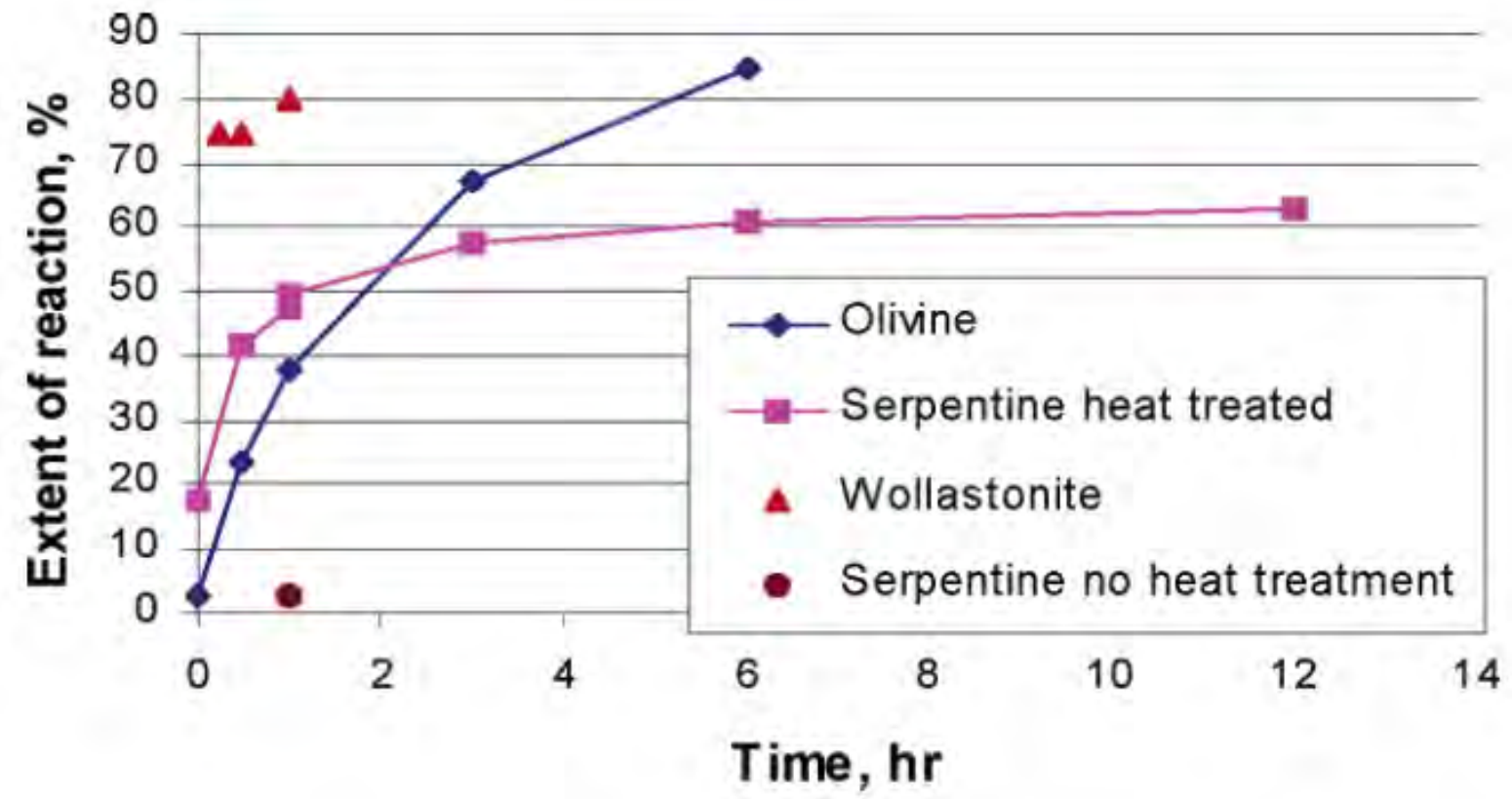

Figure 2-4: Extent of carbonation of olivine, wollastonite and serpentine at $185^{\circ} \mathrm{C}$ and $150 \mathrm{~atm} \mathrm{CO}_{2}$ in $\mathrm{NaCl} / \mathrm{NaHCO}$ solution (Gerdemann et al., 2007)

Though heat treatment significantly increased the extent of reaction, the heat treated serpentine could not exceed $65 \%$ conversion under the same operating conditions as shown in Figure 2-4. It can also be seen from this figure that wollastonite was found to be relatively more reactive in comparison to the other silicate minerals. Koukouzas et al. (2009) used dunite, hartzburgite and pyroxenite in an aqueous mineral carbonation scheme at $155^{\circ} \mathrm{C}$ and 158.6 bar using $\mathrm{NaCl} / \mathrm{NaHCO}_{3}$ solution. Though these conditions were similar to those used by Gerdemann et al. (2007), the authors reported lower $(<10 \%)$ extents of carbonation for the samples studied, with pyroxenite not carbonating at all (Koukouzas et al., 2009).

\subsubsection{Indirect Aqueous Mineral Carbonation}

This route involves at least two separate aqueous process steps carried out in different reactors. In its simplest and most generic form, the indirect aqueous mineral carbonation approach entails the reaction of aqueous solutions with silicate minerals to convert them to more reactive forms, specifically soluble cations or hydroxide compounds (mineral conversion), followed by the reaction with carbon dioxide to form stable mineral carbonates. The most attractive of these processes are those that provide for regeneration of the conversion reagent (Figure 2-5).

The biggest advantage of the indirect, multi-stage mineral carbonation approach is that it allows for individual optimisation of process steps, in isolation. 


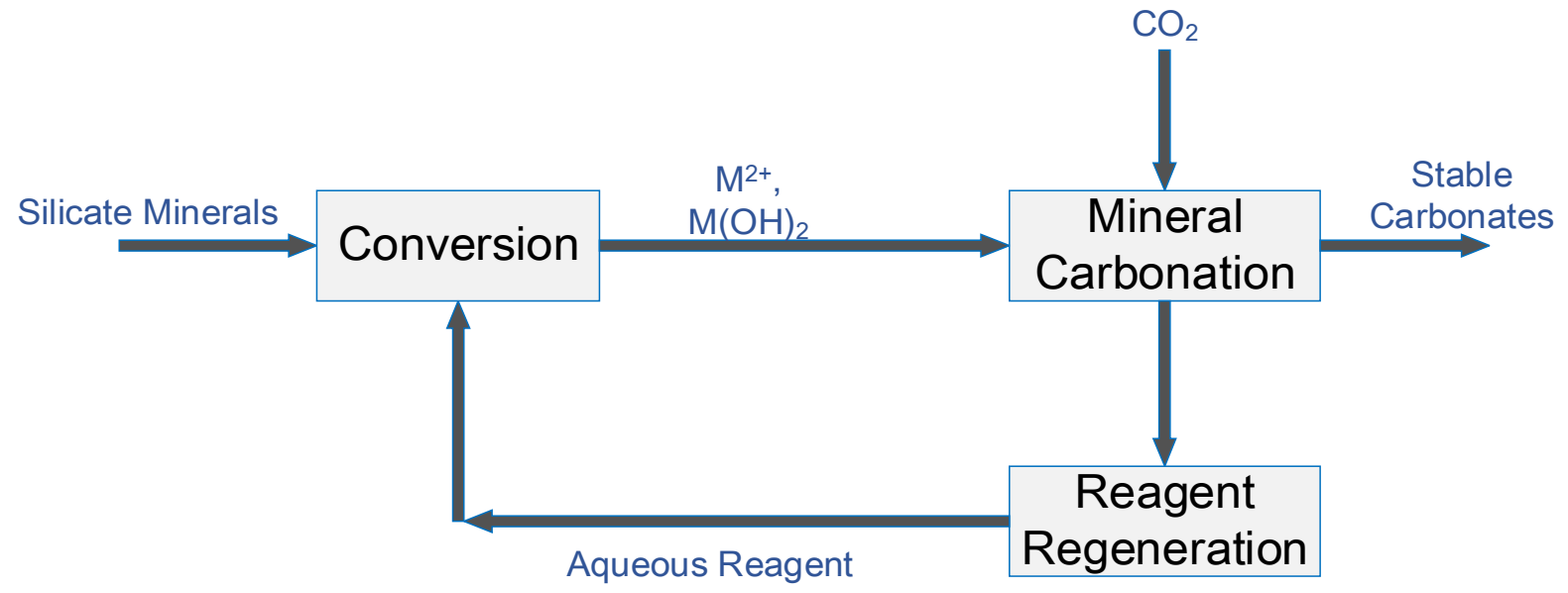

Figure 2-5: Generic indirect aqueous carbonation approach

The indirect aqueous processes reviewed here include the generic $\mathrm{pH}$ swing method, the ammonium salts process, which is a hybrid of the $\mathrm{pH}$ swing method, Lackner's multi-stage process (based on $\mathrm{HCl}$ extraction), the acetic acid process, and the alkali leach process.

\section{pH Swing Method}

The aqueous carbonation of silicate minerals is a two-step process that involves; 1) the dissolution of cations into solution under acidic conditions, and 2) the carbonation of dissolved cations in the solution under alkaline conditions. The $\mathrm{pH}$ swing method, is based on studies by Park et al. (2003) which established that the overall carbonation rate was limited by the extent of carbonic acid dissociation into bicarbonate or carbonate ions in acidic conditions. These acidic conditions enhanced the dissolution of the silicate mineral but inhibited the carbonation of magnesium ions. The carbonation reaction was favoured by high $\mathrm{pH}$ conditions, with the optimum $\mathrm{pH}$ being around 10 (Park, 2005). It was from this understanding that the authors proposed the $\mathrm{pH}$ swing method. The first step of the $\mathrm{pH}$ swing method entails the extraction of cations from the silicate minerals through dissolution, typically, in inorganic acids, organic acids, or a mixture of the two (Park and Fan, 2004; Teir et al., 2007a; Meyer et al., 2014), although ammonium salts have also been used (Wang and Maroto-Valer, 2011; Sanna et al., 2014a). The acidic leach solution is then $\mathrm{pH}$ adjusted using alkaline solutions such as sodium hydroxide, and subsequently carbonated with $\mathrm{CO}_{2}$ (Meyer et al., 2014).

A schematic illustration of a $\mathrm{pH}$ swing process is provided in Figure 2-6, and a detailed review of the mineral dissolution (extraction) and carbonation steps outlined in the sub-sections below. 


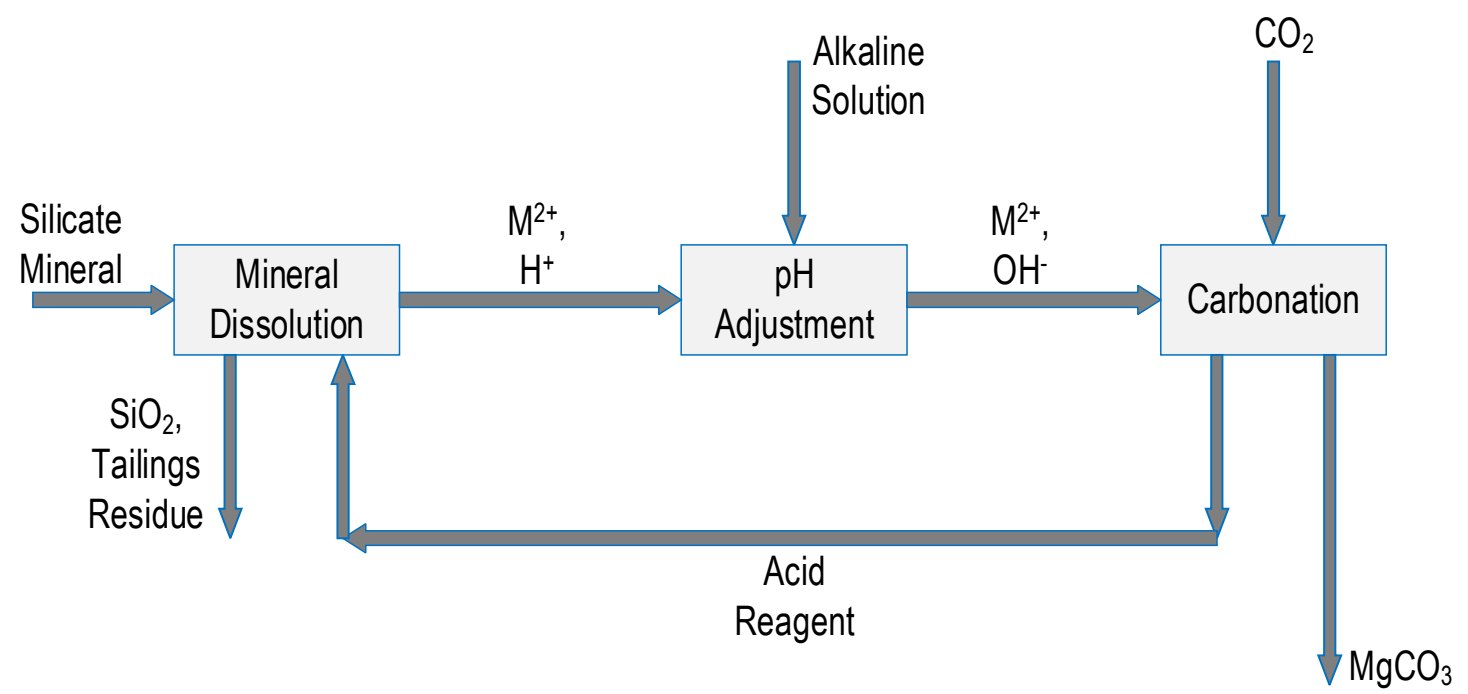

Figure 2-6: Simplified schematic of pH swing process

\section{Mineral Dissolution}

The extraction of cations from the mineral matrix is the first step in indirect aqueous carbonation, and indeed, in the $\mathrm{pH}$ swing method. This step has also been identified as rate limiting (Park and Fan., 2004; Alexander et al., 2007; Gerdemann et al., 2007; Meyer et al., 2014), and accordingly much of the research and development in this area has been focused on accelerating this step. A number of parameters affect the extraction of alkaline earth metals from the silicate minerals including solution chemistry, temperature and pressure conditions, mineral type and particle size.

\section{i. Solution Chemistry}

An important parameter that affects the indirect aqueous mineral carbonation process is the solution chemistry, particularly for the extraction of the metal cations from the silicate mineral matrix. Researchers have thus experimented with different chemical reagents, from organic to inorganic acids, to ammonium salts, as well as alkaline solutions among others. Depending on the $\mathrm{pH}$, the extraction of the silicate mineral can either be proton controlled, at low $\mathrm{pH}$ (Krevor and Lackner, 2001) or be enhanced by the presence of ligands that weaken or polarize the bond between the cation and the mineral lattice, at $\mathrm{pH}$ conditions near neutral (Brantley, 2008). This occurs when the protons from the organic acid hydrate the Si-O tetrahedral, with the ligands forming complexes at the reaction front through covalent bonding between the oxygen of the organic compound and the surface metal (Meyer, 2014). Table 2-4 is a summary of results from 
the extraction of various silicate minerals using different solutions; organic and inorganic acids, mixtures of the two, as well as ammonium salts.

Table 2-4: Summary of results from dissolution studies

\begin{tabular}{|c|c|c|c|}
\hline Study & Silicate Mineral & Solvent & $\begin{array}{l}\text { Extraction } \\
\text { efficiency }\end{array}$ \\
\hline \multirow{2}{*}{$\begin{array}{l}\text { Park and Fan } \\
\qquad(2004)\end{array}$} & \multirow{2}{*}{ Serpentine } & A mixture of $\mathrm{H}_{3} \mathrm{PO}_{4}$, oxalic acid and EDTA & $65 \%$ in $1 \mathrm{hr}$ \\
\hline & & Ammonium Bisulphate $\left(\mathrm{NH}_{4} \mathrm{HSO}_{4}\right)$ & $42 \%$ in $1 \mathrm{hr}$ \\
\hline \multirow{3}{*}{ Teir et al. (2007) } & \multirow{3}{*}{ Serpentinite } & Acid Solutions $\left(\mathrm{HCl}, \mathrm{H}_{2} \mathrm{SO}_{4}, \mathrm{HNO}_{3}, \mathrm{HCOOH}, \mathrm{CH}_{3} \mathrm{COOH}\right)$ & $3-26 \%$ in $1 \mathrm{hr}$ \\
\hline & & Alkaline Solutions $\left(\mathrm{NaOH}, \mathrm{KOH}, \mathrm{NH}_{3}\right)$ & $<0.05 \%$ in $1 \mathrm{hr}$ \\
\hline & & Ammonium Salt Solutions $\left(\mathrm{NH}_{4} \mathrm{Cl},\left(\mathrm{NH}_{4}\right)_{2} \mathrm{SO}_{4}, \mathrm{NH}_{4} \mathrm{NO}_{3}\right)$ & $0.3-0.5 \%$ in $1 \mathrm{hr}$ \\
\hline \multirow{4}{*}{$\begin{array}{l}\text { Wang and } \\
\text { Maroto-Valer } \\
\quad(2011)\end{array}$} & \multirow{4}{*}{ Serpentine } & Ammonium Bisulphate $\left(\mathrm{NH}_{4} \mathrm{HSO}_{4}\right)$ & $>50 \%$ in $3 \mathrm{hrs}$ \\
\hline & & Ammonium Chloride $\left(\mathrm{NH}_{4} \mathrm{Cl}\right)$ & $<5 \%$ in $3 \mathrm{hrs}$ \\
\hline & & Ammonium Sulphate $\left(\left(\mathrm{NH}_{4}\right)_{2} \mathrm{SO}_{4}\right)$ & $<5 \%$ in $3 \mathrm{hrs}$ \\
\hline & & Sulphuric Acid $\left(\mathrm{H}_{2} \mathrm{SO}_{4}\right)$ & $\sim 45 \%$ in $3 \mathrm{hrs}$ \\
\hline \multirow{2}{*}{$\begin{array}{l}\text { Meyer et al. } \\
\text { (2014) }\end{array}$} & \multirow{2}{*}{ PGM Tailings } & A mixture of $\mathrm{HCl}$, oxalic acid and EDTA & $<4.5 \%$ in $5 \mathrm{hrs}$ \\
\hline & & Hydrochloric Acid $(\mathrm{HCl})$ & $<5 \%$ in $8 \mathrm{hrs}$ \\
\hline \multirow{3}{*}{ Meyer (2014) } & enstatite & \multirow{3}{*}{$0.1 \mathrm{M} \mathrm{HCl}$} & $<2.1 \%$ in $48 \mathrm{hrs}$ \\
\hline & augite & & $<14 \%$ in $48 \mathrm{hrs}$ \\
\hline & diopside & & $3.3 \%$ in $48 \mathrm{hrs}$ \\
\hline
\end{tabular}

In one of the initial studies in this approach, Park and Fan (2004) investigated the dissolution of serpentine in a fluidized reaction system with internal grinding. The solvents used were 1.4 $\mathrm{M}$ ammonium bisulphate $\left(\mathrm{NH}_{4} \mathrm{HSO}_{4}\right)$ and a mixture of acids $\left(1 \mathrm{vol} \% \mathrm{H}_{3} \mathrm{PO}_{4}, 0.9 \mathrm{wt} \%\right.$ oxalic acid and $0.1 \mathrm{wt} \%$ EDTA solution). Ambient pressure and moderate temperature $\left(70{ }^{\circ} \mathrm{C}\right)$ conditions were used by the authors (Park and Fan, 2004). The researchers reported that mixture of acids was more effective at leaching magnesium resulting in a $65 \%$ extraction efficiency in comparison to $42 \%$ extraction achieved using the $1.4 \mathrm{M}$ solution of ammonium bisulphate. Teir et al. (2007a) investigated the effect of different concentrations ( $1 \mathrm{M}, 2 \mathrm{M}, 4$ M) of various solvents on the extraction of magnesium from a 74-125 $\mu \mathrm{m}$ sample of serpentinite at temperatures of $30{ }^{\circ} \mathrm{C}, 50^{\circ} \mathrm{C}$ and $70{ }^{\circ} \mathrm{C}$. The solvents they considered included inorganic acids $\left(\mathrm{H}_{2} \mathrm{SO}_{4}, \mathrm{HCl}, \mathrm{HNO}_{3}\right)$, organic acids $\left(\mathrm{HCOOH}, \mathrm{CH}_{3} \mathrm{COOH}\right)$, alkaline solutions $\left(\mathrm{NaOH}, \mathrm{KOH}, \mathrm{NH}_{3}\right)$, as well as various ammonium salts $\left(\mathrm{NH}_{4} \mathrm{Cl},\left(\mathrm{NH}_{4}\right)_{2} \mathrm{SO}_{4}, \mathrm{NH}_{4} \mathrm{NO}_{3}\right)$. Their findings, summarised in Figure 2-7, demonstrated that $\mathrm{H}_{2} \mathrm{SO}_{4}$ was the most effective solvent for the dissolution of serpentinite. 


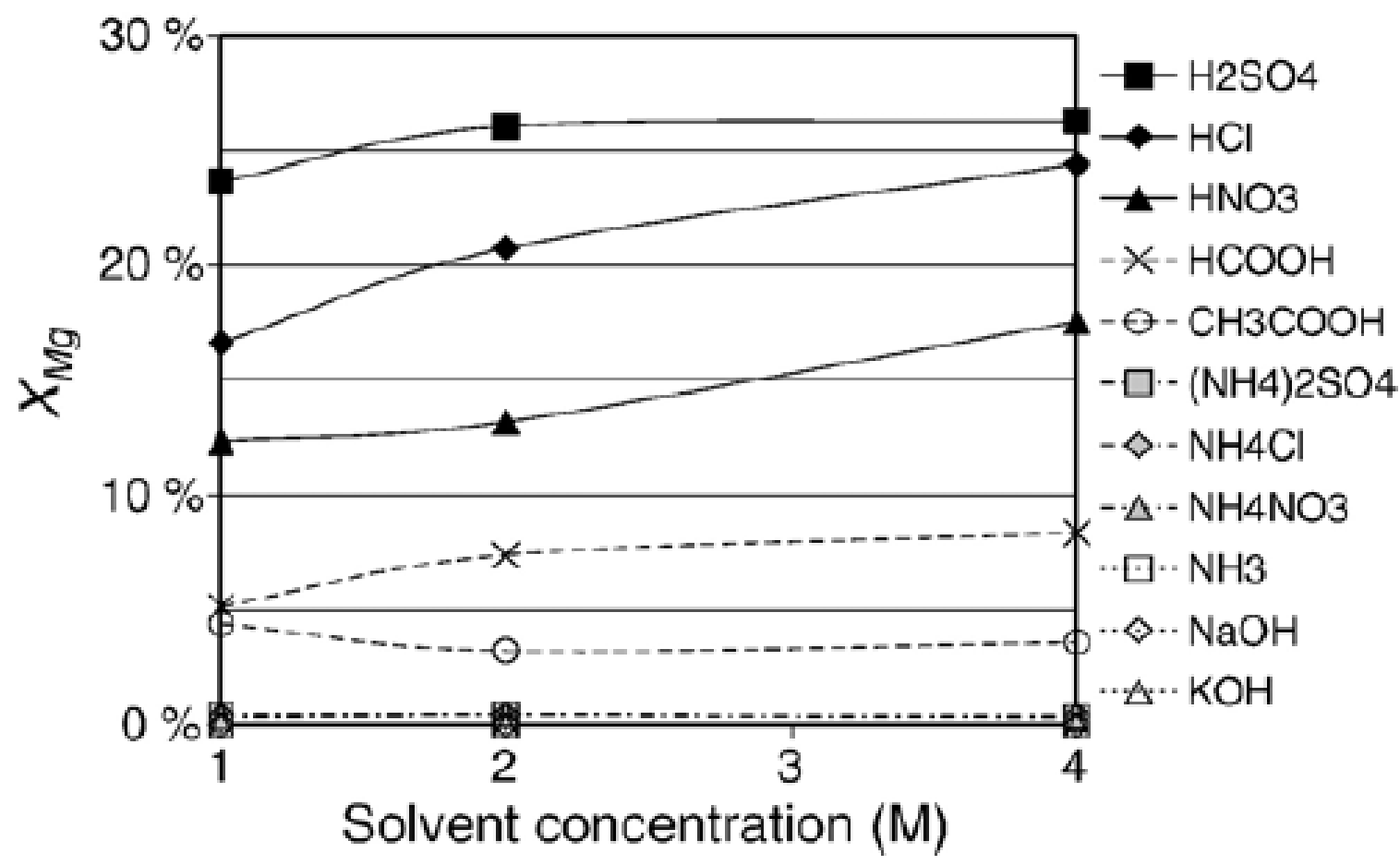

Figure 2-7: Extraction efficiency of magnesium from serpentinite in $1 \mathrm{M}, 2 \mathrm{M}, 4 \mathrm{M}$ solutions at $20^{\circ} \mathrm{C}$ in $1 \mathrm{hr}$ (Teir et al., 2007a)

Overall, the study found the most effective solvents were inorganic acids followed by organic acids. Ammonium salts, though selectively extracting magnesium, had lower extraction ratios. Finally, the alkaline solutions tested yielded no measureable amount of cation extraction. In their work Teir et al. (2007) constrained the dissolution experiments to a 1-hour timeframe. The use of longer time-frames ( $\geq 6$ hours) would have been more illuminating, especially with regard to the selectivity of ammonium salts.

A study by Wang and Maroto-Valer (2011) compared the rate of extraction of magnesium from serpentine using $\left(\mathrm{NH}_{4}\right)_{2} \mathrm{SO}_{4}, \mathrm{NH}_{4} \mathrm{Cl}$ and $\mathrm{NH}_{4} \mathrm{HSO}_{4}$, and $\mathrm{H}_{2} \mathrm{SO}_{4}$ under different conditions. The use of $\mathrm{H}_{2} \mathrm{SO}_{4}$ was to compare the performance of the ammonium salts against a strong inorganic acid that was effective in mineral dissolution (Teir et al., 2007). The use of ammonium salts provides opportunities to recycle dissolution reactants (Maroto-Valer and Wang, 2011). Results, summarised in Figure 2-8, indicated that ammonium bisulphate $\left(\mathrm{NH}_{4} \mathrm{HSO}_{4}\right)$ extracted the most magnesium from serpentine at $70{ }^{\circ} \mathrm{C}$ in 3 hours $(50 \%)$, followed by $\mathrm{H}_{2} \mathrm{SO}_{4}(45 \%)$. $\mathrm{NH}_{4} \mathrm{Cl}$ and $\left(\mathrm{NH}_{4}\right)_{2} \mathrm{SO}_{4}$ resulted in low extraction efficiencies $(<5 \% \mathrm{Mg}$ extraction). The results obtained by Wang and Maroto-Valer (2011) were consistent with those obtained by Teir et al (2007a) and Park and Fan (2004). 


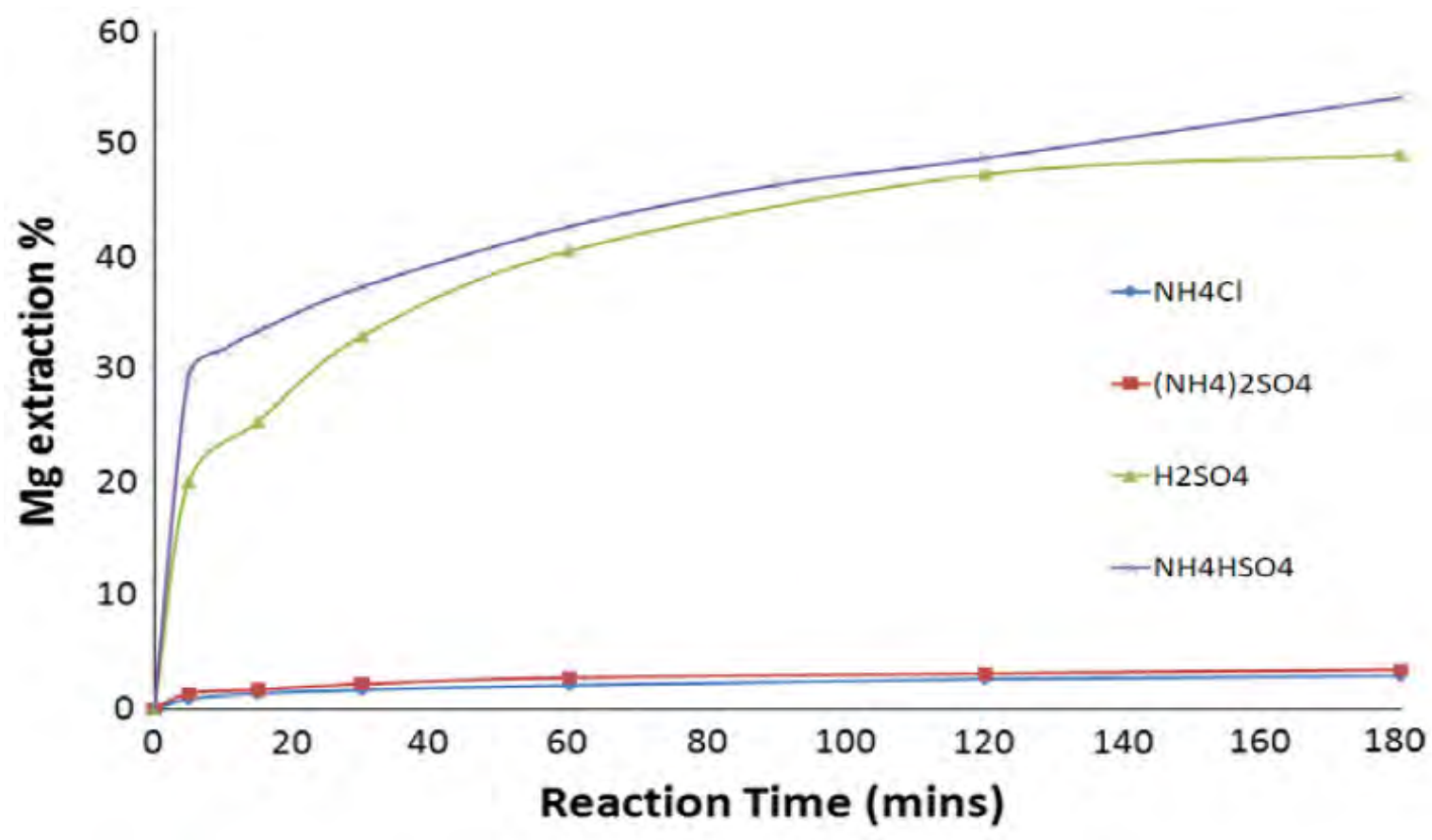

Figure 2-8: Extraction efficiency of magnesium from serpentinite in ammonium salt and sulphuric acid solutions at $70^{\circ} \mathrm{C}$ in 3 hrs (Wang and Maroto-Valer, 2011)

It is important to note that $2 \mathrm{M} \mathrm{NH}_{4} \mathrm{HSO}_{4}$ was used in the study by Wang and Maroto-Valer (2011) in comparison to the $1.4 \mathrm{M} \mathrm{NH}_{4} \mathrm{HSO}_{4}$ solvent used by Park and Fan (2004), which suggests that extraction of cations is independent of acid concentration at these molarities for this particular solvent.

To investigate the mineral carbonation of pyroxene-rich PGM tailings, Meyer et al. (2014) used an inorganic acid $(2 \mathrm{M} \mathrm{HCl})$ and mixtures of 0.06-0.09 $\mathrm{M}$ hydrochloric acid, 0.01-0.04 M EDTA and 0.001-0.1 M oxalic acid for dissolution at $70{ }^{\circ} \mathrm{C}$. The authors noted that this mixture of acids solvent did not increase extraction of magnesium as reported by Park et al. (2003).

\section{Temperature}

In dissolution studies by Teir et al., (2007a) discussed in $(i)$ the researchers also conducted experiments to determine the effect of temperature on extraction. The authors reported an increase in magnesium dissolution from serpentinite with an increase in temperature from 30 ${ }^{\circ} \mathrm{C}$ to $70{ }^{\circ} \mathrm{C}$ using $2 \mathrm{M}$ solutions of sulphuric acid $\left(\mathrm{H}_{2} \mathrm{SO}_{4}\right)$, hydrochloric acid $(\mathrm{HCl})$ and nitric acid $\left(\mathrm{HNO}_{3}\right)$. Figure 2-9 shows the effect of temperature on the dissolution of magnesium and iron in $2 \mathrm{M} \mathrm{H}_{2} \mathrm{SO}_{4}$ solution. It is noted that $100 \%$ extraction of magnesium was obtained at 70 ${ }^{\circ} \mathrm{C}$. Similar results were also reported for $\mathrm{HCl}$ and $\mathrm{HNO}_{3}$ solutions (Teir et al., 2007a). 


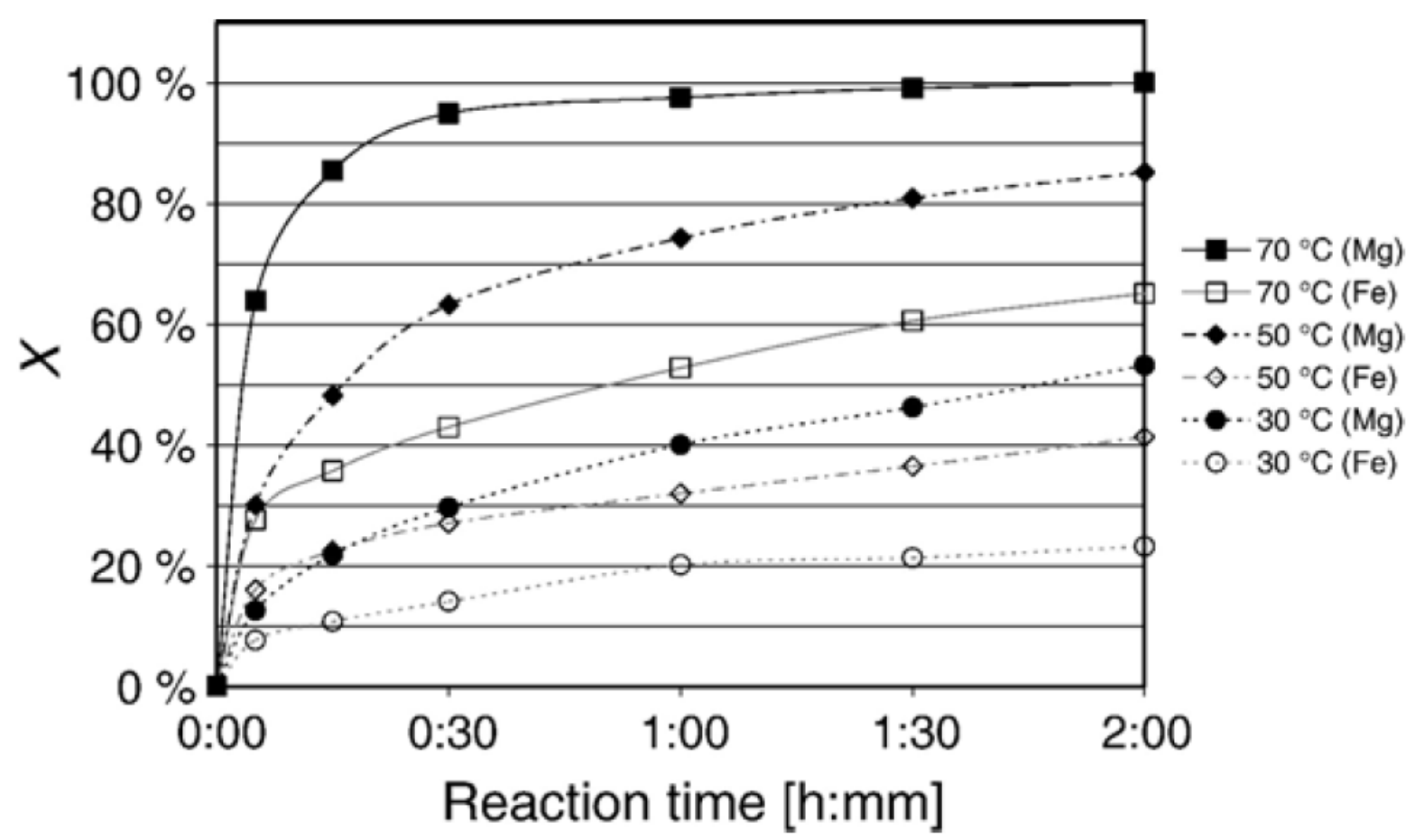

Figure 2-9: Effect of temperature on extent of dissolution of $\mathrm{Mg}$ and Fe in $2 \mathrm{M} \mathrm{H}_{2} \mathrm{SO}_{4}$ (Teir et al., 2007a)

Prigiobbe and Mazzotti (2011) investigated the effect of temperature in the dissolution of olivine in sodium salts (oxalate and citrate). They reported enhanced dissolution of olivine with increased temperatures up to $120^{\circ} \mathrm{C}$, demonstrated by the increase in the specific dissolution rate. Another author in the same research group, Hanchen et al. (2006), had earlier conducted dissolution experiments on olivine (forsterite end-member), using hydrochloric acid at temperatures from $90-150{ }^{\circ} \mathrm{C}$. Their results also indicated an increase in dissolution with an increase in temperature, with dissolution rates of $2.2 \mathrm{e}-10$ to $3.76 \mathrm{e}-9 \mathrm{~mol} \cdot \mathrm{cm}^{-2} \cdot \mathrm{s}^{-1}$ at temperatures of $90^{\circ} \mathrm{C}$ and $150{ }^{\circ} \mathrm{C}$, respectively.

Alexander et al. (2007) reported a 70\% increase in magnesium extraction from serpentine by sulphuric acid with an increase in temperature from $25{ }^{\circ} \mathrm{C}$ to $50{ }^{\circ} \mathrm{C}$, whilst studies by Wang and Maroto-Valer (2011) showed that magnesium extraction efficiencies in a $1.4 \mathrm{M}$ ammonium bisulphate solution increased, on increasing the temperature in the range $70{ }^{\circ} \mathrm{C}$ to $100{ }^{\circ} \mathrm{C}$. In accordance with the latter studies, summarized in Figure 2-10, the effect of temperature becomes more pronounced at higher temperatures, with the extraction efficiency approaching $100 \%$ at $100{ }^{\circ} \mathrm{C}$. 


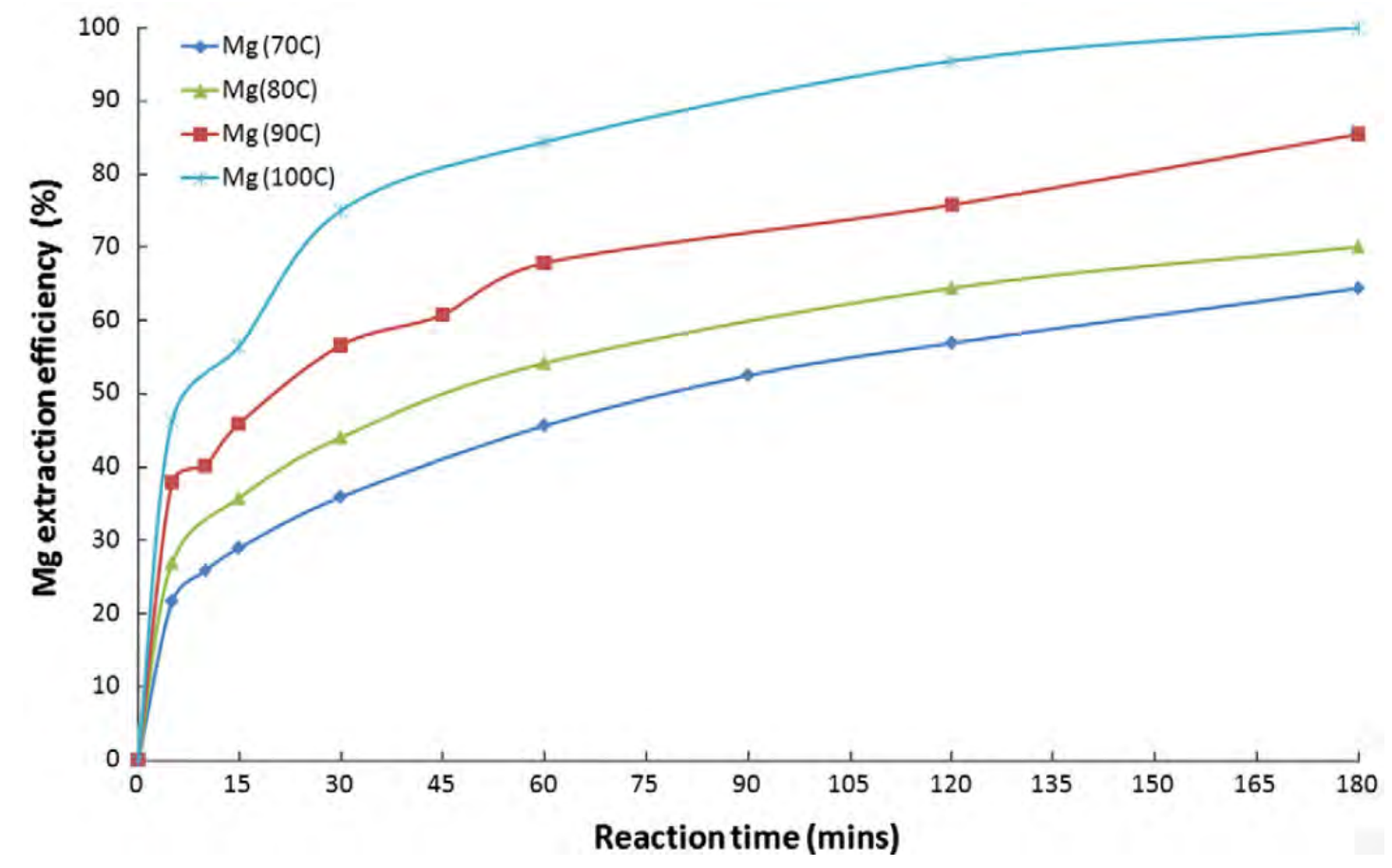

Figure 2-10: Effect of temperature on the dissolution of serpentine in 1.4 $\mathrm{M} \mathrm{NH}_{4} \mathrm{HSO}_{4}$ solution (Wang and Maroto-Valer, 2011)

A similar study conducted by Sanna et al. (2014a) on pyroxene in 1.4 M ammonium bisulphate exhibited similar, though more modest increases in extraction efficiencies on increasing the temperature from $50{ }^{\circ} \mathrm{C}$ to $100{ }^{\circ} \mathrm{C}$ (Figure 2-11).

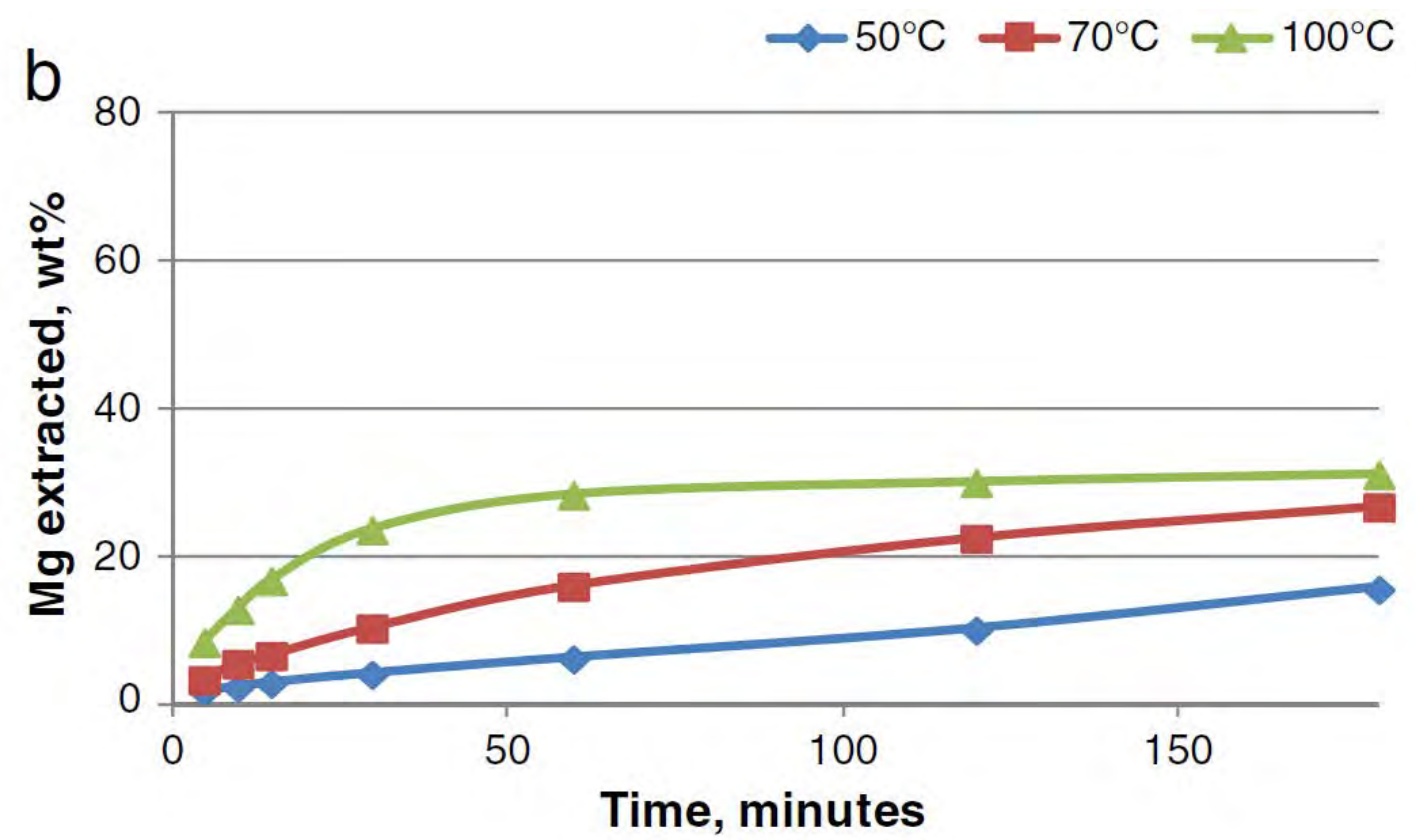

Figure 2-11: Effect of temperature on the dissolution of pyroxene in $1.4 \mathrm{M} \mathrm{NH}_{4} \mathrm{HSO}_{4}$ solution (Sanna et al., 2014a) 


\section{ii. Mineral Type}

A review of results indicates that the rate and extent of dissolution is highly dependent on the structure, and hence type of mineral (see results in Table 2-5).

Table 2-5: Impact of mineral type on magnesium extraction under the same conditions

\begin{tabular}{|c|c|c|c|c|}
\hline Study & Silicate Mineral & Solvent & Conditions & Mg-Extraction efficiency \\
\hline \multirow{3}{*}{ Sanna et al. (2014) } & Olivine & \multirow{3}{*}{$1.4 \mathrm{M} \mathrm{NH}_{4} \mathrm{HSO}_{4}$} & \multirow{3}{*}{$100^{\circ} \mathrm{C}, 1 \mathrm{~atm}$} & $78 \%$ in $3 \mathrm{hrs}$ \\
\hline & Pyroxene & & & $31 \%$ in $3 \mathrm{hrs}$ \\
\hline & Amphibole Rocks & & & $29 \%$ in $3 \mathrm{hrs}$ \\
\hline \multirow{3}{*}{ Meyer (2014) } & enstatite & \multirow{3}{*}{$0.1 \mathrm{M} \mathrm{HCl}$} & \multirow{3}{*}{$40^{\circ} \mathrm{C}, 1 \mathrm{~atm}$} & $<2.1 \%$ in $48 \mathrm{hrs}$ \\
\hline & augite & & & $<14 \%$ in $48 \mathrm{hrs}$ \\
\hline & diopside & & & $3.3 \%$ in $48 \mathrm{hrs}$ \\
\hline
\end{tabular}

The study by Sanna et al. (2014a) showed that olivine was considerably more reactive than pyroxene and amphibole-rich rocks, with a $1.4 \mathrm{M}$ bisulphate solution resulting in $\mathrm{Mg}$ extraction of $77 \%$ from olivine in 3 hours at $100{ }^{\circ} \mathrm{C}$ compared to approximately $30 \% \mathrm{Mg}$ extraction from pyroxene and amphibole under the same conditions. In accordance with the study by Meyer (2014) reactivity of silicate minerals in dilute $\mathrm{HCl}$ solutions at $40{ }^{\circ} \mathrm{C}$ decreased in the order, wollastonite $>$ diopside $=$ augite $>$ enstatite. Meyer attributed this reactivity to the structure of the minerals, particularly in terms of the length of the metal-oxygen bonds and the size and location of the metal cations in the mineral structure (Meyer, 2014). In order to increase Mg-extraction in pyroxene samples researchers have suggested the application of mechanical activation, higher temperatures and pressures, exploring different leach reagents as well as increased reaction times (Koukouzas et al., 2009; Meyer et al., 2014). These tailings are mostly orthopyroxene (65 wt.\%) and plagioclase (16 wt.\%) with clinopyroxene and amphibole in smaller amounts (Meyer et al., 2014). The authors obtained relatively low extraction of magnesium $(<4.8 \%)$ in comparison to that reported by other authors using similar solvents to extract cations from more reactive serpentine minerals (Park and Fan, 2004; Teir et al, 2007a; Wang and Maroto-Valer, 2011). Meyer (2014) conducted dissolution experiments on pure pyroxene minerals (augite, wollastonite, enstatite and diopside), at temperatures between ambient and $70{ }^{\circ} \mathrm{C}$, using $0.1 \mathrm{M}$ hydrochloric acid. The extraction efficiency of magnesium in all these minerals was reported to be low, with the highest attained efficiency being $12 \%$ extracted from augite. 


\section{Carbonation}

The next major process step in the $\mathrm{pH}$ swing method, is the carbonation of cations in solution, under alkaline conditions. Park and Fan (2004) used ammonium hydroxide $\left(\mathrm{NH}_{4} \mathrm{OH}\right)$ to increase the $\mathrm{pH}$ to 9.5 , at which point the precipitation of magnesium carbonate occurred at ambient temperature conditions. According to these researchers, the precipitation of magnesium carbonate was instantaneous at the desired alkaline $\mathrm{pH}$ conditions resulting in complete conversion of the dissolved magnesium in solution in both solvent cases. In their studies, Teir et al. (2007b) used sodium hydroxide $(\mathrm{NaOH})$ to adjust the $\mathrm{pH}$, to an optimum carbonation $\mathrm{pH}$ of 9 . The authors reported carbonation efficiencies of up to $94 \%$ at $30{ }^{\circ} \mathrm{C}$, producing a hydromagnesite product that is up to 99\% pure. Meyer et al. (2014) also used the same $\mathrm{pH}$ conditions and chemical reagent $(\mathrm{NaOH})$ in carbonation of cation solutions produced through dissolution by $2 \mathrm{M} \mathrm{HCl}$. The authors reported "rapid and efficient" carbonation under these conditions. Wang and Maroto-Valer (2013) reported carbonation efficiencies of more than $90 \%$ at $80{ }^{\circ} \mathrm{C}$ using ammonium bicarbonate, produced from carbon dioxide capture with $\mathrm{NH}_{4} \mathrm{OH}$ to carbonate the cation solution.

\section{Case Study Examples of the two-stage $\mathrm{pH}$ swing process}

Implementing the $\mathrm{pH}$ swing method to their work on serpentinite, a rock primarily dominated by serpentine group minerals, Teir et al. (2007b) conducted dissolution experiments using solutions of hydrochloric acid and nitric acid at $70{ }^{\circ} \mathrm{C}$ (Equations 13 and 14).

$$
\begin{gathered}
\mathrm{Mg}_{3} \mathrm{Si}_{2} \mathrm{O}_{5}(\mathrm{OH})_{4(\mathrm{~s})}+6 \mathrm{HCl}_{(\mathrm{aq})} \rightarrow 3 \mathrm{MgCl}_{2(\mathrm{aq})}+2 \mathrm{SiO}_{2(\mathrm{~s})}+5 \mathrm{H}_{2} \mathrm{O}_{(\mathrm{l})} \\
\mathrm{Mg}_{3} \mathrm{Si}_{2} \mathrm{O}_{5}(\mathrm{OH})_{(\mathrm{s})}+6 \mathrm{HNO}_{3(\mathrm{aq})} \rightarrow 3 \mathrm{Mg}\left(\mathrm{NO}_{3}\right)_{2(\mathrm{aq})}+2 \mathrm{SiO}_{2(\mathrm{~s})}+5 \mathrm{H}_{2} \mathrm{O}_{(\mathrm{l})}
\end{gathered}
$$

The authors found relatively high extraction efficiencies for both hydrochloric acid and nitric acid at $92.6 \%$ and $88.3 \%$, respectively. The dissolution experiments were followed up by further testwork to establish the optimum $\mathrm{pH}$ for the precipitation of magnesium ions into carbonate through carbonation at $30{ }^{\circ} \mathrm{C}$ and $1 \mathrm{~atm}$. The leach solutions produced were carbonated at varied $\mathrm{pH}$ conditions and the optimum carbonation $\mathrm{pH}$ was reported to be $\mathrm{pH} 9$, which gives the highest amount of carbon dioxide fixed as carbonate as indicated in Figure 2-12. From this figure it can be also seen that this $\mathrm{pH}$ also provided the highest purity of hydromagnesite (99\%). 


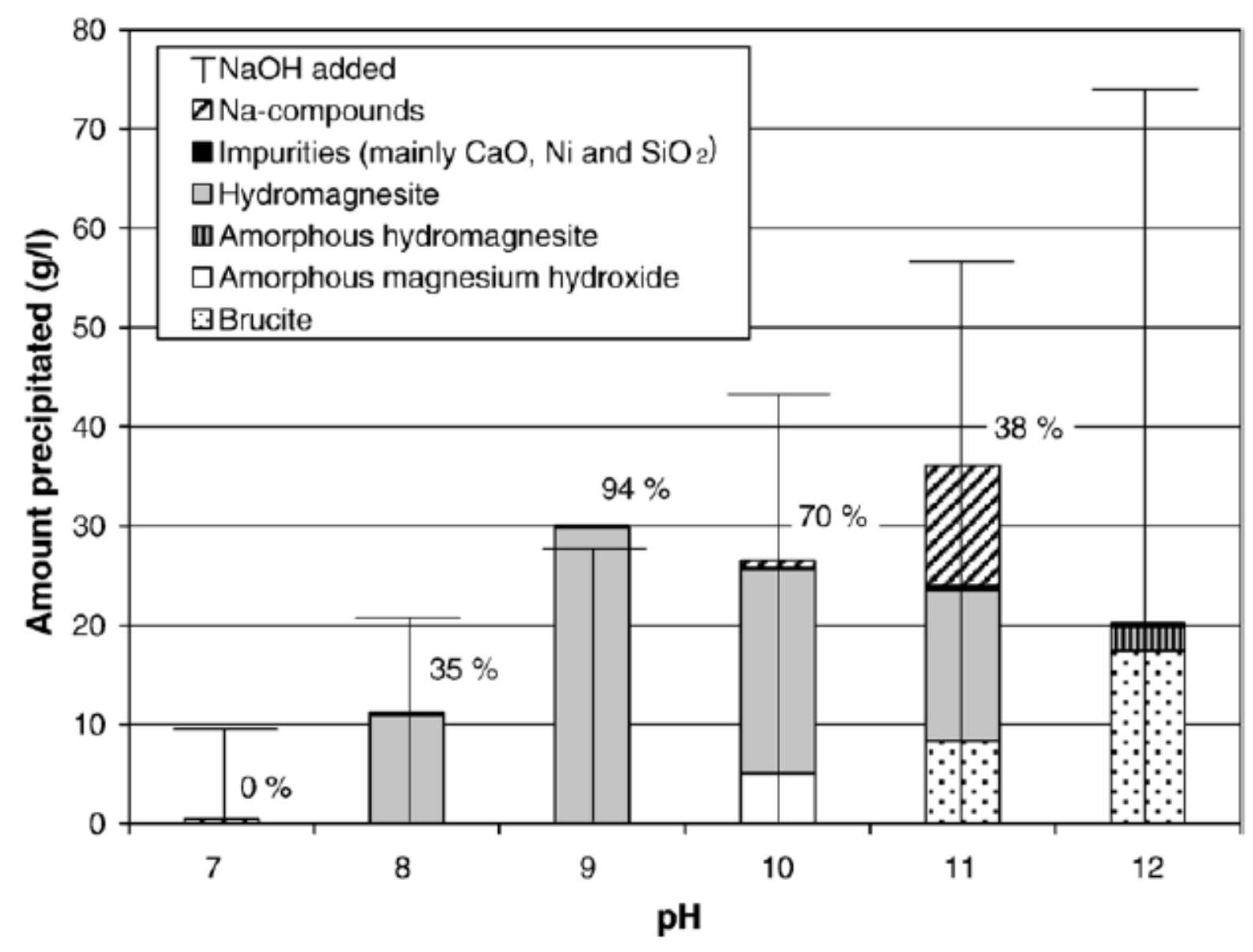

Figure 2-12: Results from the conversion of magnesium ions into carbonate, effect of pH (Teir et al., 2007b)

This work served as a basis for the development of a conceptual plant for the mineral carbonation of serpentinite (Teir et al., 2009). Upon dissolution the authors proposed the evaporation of water to crystallise the nitrate and chloride salts of magnesium (Teir et al., 2007b; Teir et al., 2009). The addition of sodium hydroxide and water dissolves the salts and neutralises the solution, also precipitating any iron oxides present. The solution is finally carbonated to produce hydromagnesite through the addition of more sodium hydroxide and bubbling carbon dioxide through the solution. A simple flowsheet for the proposed process is presented in Figure 2-13. The authors found it would require 3.1 tons of serpentine to store a ton of carbon dioxide. It was also found that significant quantities of acid and sodium hydroxide are required, and that potential recycling of these reagents could require energy intensive operations like electrolysis (Teir et al., 2009). 


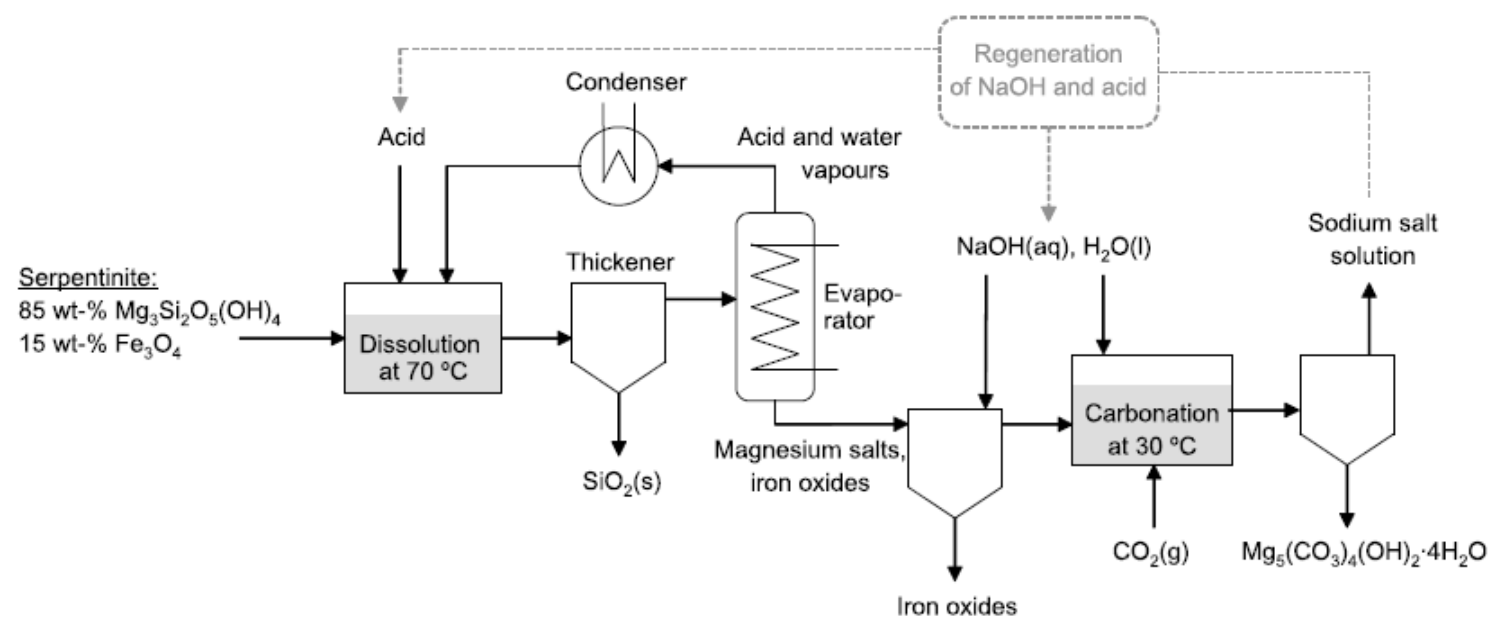

Figure 2-13: A simple flowsheet for the carbonation of serpentinite through a pH swing method using $\mathrm{HCl} / \mathrm{HNO}$ and $\mathrm{NaOH}$ (Teir et al., 2009)

A similar approach was taken in studies conducted on pyroxene-rich PGM tailings (Vogeli, 2012; Meyer et al., 2014). The authors adopted the $\mathrm{pH}$ swing process to the dissolution and carbonation of cations in pyroxene-rich PGM tailings. After a classification study to ascertain the potential of PGM tailings to sequester carbon dioxide, Vogeli (2012) used 2 M hydrochloric acid to leach different samples of PGM tailings at $70{ }^{\circ} \mathrm{C}$. The resulting leach solution was subsequently $\mathrm{pH}$ adjusted to $\mathrm{pH} 9$ using $15 \mathrm{M}$ sodium hydroxide prior to carbonation at $20{ }^{\circ} \mathrm{C}$. In contrast to the process proposed by Teir et al. (2007), the evaporation of the acid solution for recycle prior to $\mathrm{pH}$ adjustment was not part of this approach. Comparatively modest extraction efficiencies were reported $(\sim 20 \%)$ in comparison to other silicate minerals studied under the same conditions, as reported by Teir et al. (2007b) discussed earlier. The carbonation efficiency of the $\mathrm{pH}$ adjusted leach solution was reported to range between $48 \%$ and $69 \%$ (Vogeli, 2012). Meyer et al. (2014) reported findings that reaffirmed observations that mineral dissolution is the rate limiting step in aqueous mineral carbonation (Park and Fan, 2004; Wang and Maroto-Valer, 2011; Bonfils et al., 2012) since subsequent carbonation was "rapid and efficient".

\section{The Ammonium Salts Process}

In a modified version of the $\mathrm{pH}$-swing method, Wang and Maroto-Valer (2011) proposed a multi-stage ammonium salts process to capture carbon dioxide, extract and carbonate the silicate mineral. This process was developed based on a patent for the production of silica, iron oxide and magnesium carbonate from serpentine wastes for economic purposes (Pundsack et al., 1967). The process is described below through key chemical equations and operations, and presented schematically in Figure 2-14: 
1. $\mathrm{CO}_{2}$ Capture: The ammonium salts process integrates carbon dioxide capture from flue gases as part of the carbonation process. Hydrated ammonia is used to capture ambient pressure carbon dioxide producing ammonium bicarbonate at $10{ }^{\circ} \mathrm{C}$. This is claimed to have the benefit of resulting in energy savings by avoiding compression and transport of carbon dioxide (Wang and Maroto-Valer, 2011).

$$
\mathrm{NH}_{3(\mathrm{~g})}+\mathrm{CO}_{2(\mathrm{~g})}+\mathrm{H}_{2} \mathrm{O}_{(\mathrm{l})} \rightarrow \mathrm{NH}_{4} \mathrm{HCO}_{3(\mathrm{aq})}
$$

2. Mineral Dissolution: A 1.4 M solution of ammonium bisulphate is then used, in excess of $40 \%$, as the leaching agent to extract magnesium from serpentine at $90{ }^{\circ} \mathrm{C}$, producing a solution of magnesium and ammonium sulphate as well as solid silicon dioxide. This, the authors assert, enables the recovery of pure silica which can be of commercial benefit (Wang et al., 2013).

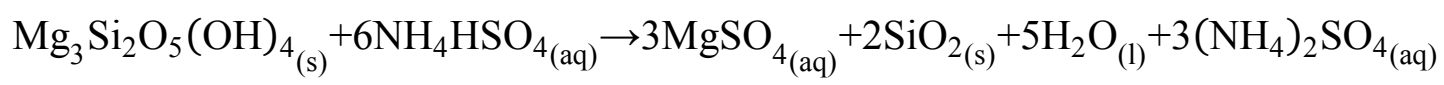

3. $p H$ adjustment: Since $40 \%$ excess ammonium bisulphate is used in the dissolution step, ammonium hydroxide is added to neutralise this excess acid at $25^{\circ} \mathrm{C}$. The product of this reaction is ammonium sulphate, which can be decomposed to reproduce key reagents in the process.

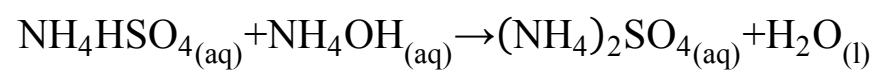

4. Carbonation: Using ammonium carbonate from the capture step as the $\mathrm{CO}_{2}$-carrier, magnesium sulphate leach solution is carbonated at $80{ }^{\circ} \mathrm{C}$ to produce hydromagnesite.

$$
\mathrm{MgSO}_{4(\mathrm{aq})}+2 \mathrm{NH}_{4} \mathrm{HCO}_{3(\mathrm{aq})}+2 \mathrm{H}_{2} \mathrm{O}_{(\mathrm{l})} \rightarrow \mathrm{MgCO}_{3} \cdot 6 \mathrm{H}_{2} \mathrm{O}_{(\mathrm{s})}+\left(\mathrm{NH}_{4}\right)_{2} \mathrm{SO}_{4(\mathrm{aq})}+\mathrm{CO}_{2(\mathrm{~g})}
$$

5. Reagent Regeneration: Some preceding process steps (dissolution, $\mathrm{pH}$ adjustment, carbonation) in the process produce ammonium sulphate in solution. To regenerate chemical reagents, the water is evaporated leaving behind solid ammonium sulphate crystals. Solid ammonium sulphate is then thermally decomposed $\left(\mathrm{T}>300^{\circ} \mathrm{C}\right)$ evolving gaseous ammonia and leaving behind an ammonium bisulphate solid residue (Wang, 2011). These can then be recycled for use in the capture and dissolution steps, respectively.

$$
\left(\mathrm{NH}_{4}\right)_{2} \mathrm{SO}_{4(\mathrm{~s})} \rightarrow \mathrm{NH}_{4} \mathrm{HSO}_{4(\mathrm{~s})}+\mathrm{NH}_{3(\mathrm{~g})}
$$




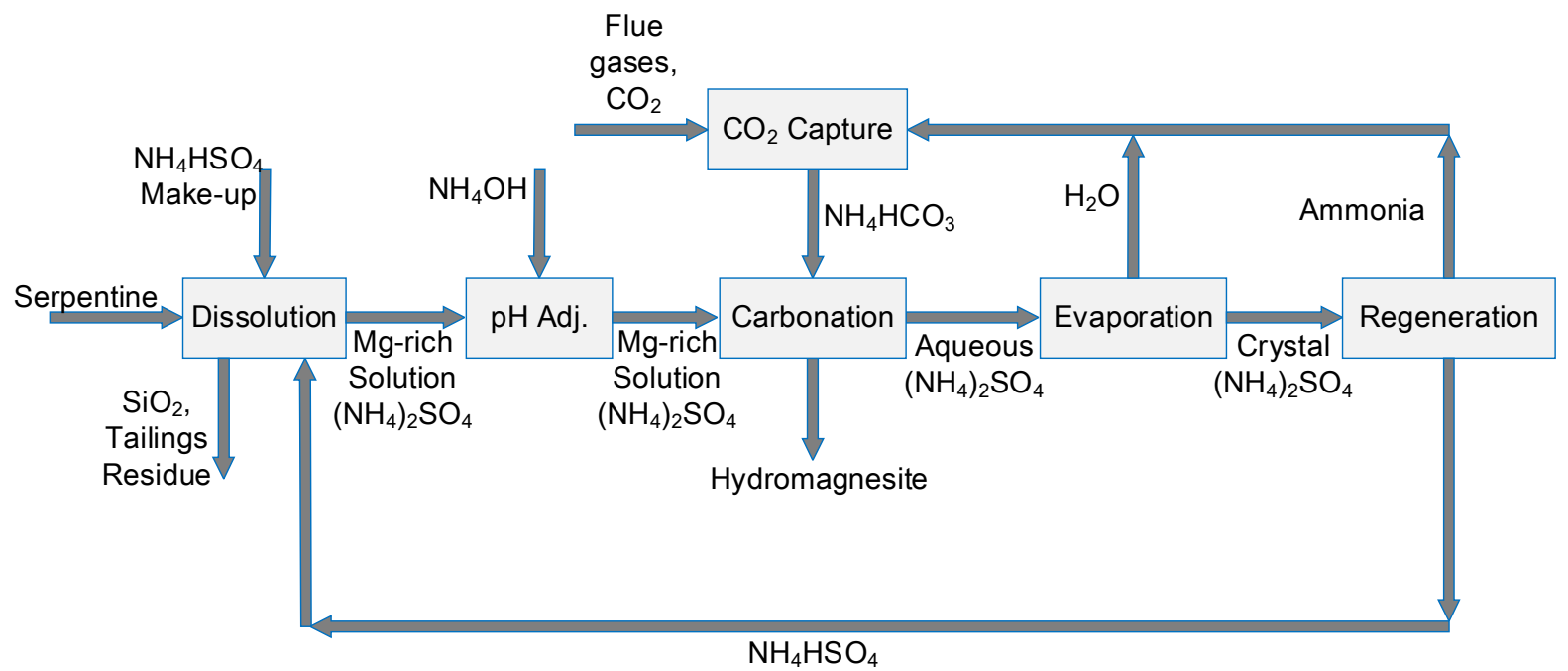

Figure 2-14: Process schematic of recyclable ammonium salts process (adapted from Wang and Maroto-Valer, 2013)

The researchers have reported magnesium extraction efficiencies of up to $100 \%$ from serpentine at $100{ }^{\circ} \mathrm{C}$ in 3 hours, and carbonation efficiency of up to $95.9 \%$ through laboratory conducted experiments (Wang et al, 2013). This signifies potential for the recyclable ammonium salts process, in that it also integrates $\mathrm{CO}_{2}$ capture. However, the recovery of reagents through thermal decomposition has potential to incur substantial energy penalties. The requirement of prior removal of water to crystallize ammonium sulphate and the high temperatures required in the endothermic decomposition reaction is concerning from an energy perspective.

\section{Lackner's Multi-stage Process}

Based on a process developed during World War II, this method involves the use of hydrochloric acid to extract metal cations from the mineral matrix. The cations are then converted to more reactive hydroxides which are then reacted with gaseous $\mathrm{CO}_{2}$. This approach was first proposed by Lackner et al. (1995) for the purposes of mineral carbonation. A simplified flowsheet of this process is provided in Figure 2-15. This was a conceptual process not with no known experimental work conducted by the authors to validate its feasibility. 


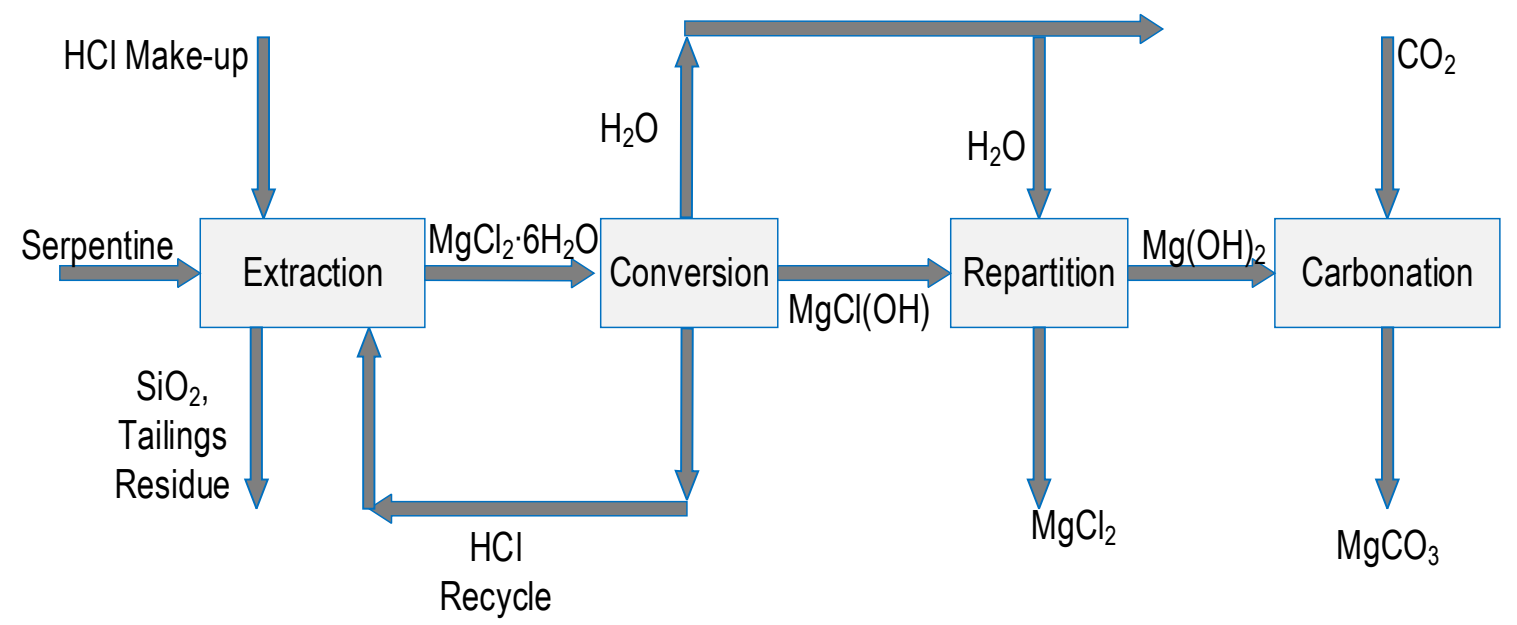

Figure 2-15: Simplified schematic of Lackner's multi-stage process

The extraction of magnesium from serpentine using $\mathrm{HCl}$ would proceed according to the following steps, as an example (Rackley, 2010):

Step 1: Extraction of magnesium as $\mathrm{MgCl}_{2}$ from serpentine. The magnesium is extracted from serpentine through the use of excess hydrochloric acid to produce $\mathrm{MgCl}_{2} \cdot 6 \mathrm{H}_{2} \mathrm{O}$.

$$
\mathrm{Mg}_{3} \mathrm{Si}_{2} \mathrm{O}_{5}(\mathrm{OH})_{4(\mathrm{~s})}+6 \mathrm{HCl}_{(\mathrm{aq})}+13 \mathrm{H}_{2} \mathrm{O}_{(\mathrm{l})} \rightarrow 3 \mathrm{MgCl}_{2} \cdot 6 \mathrm{H}_{2} \mathrm{O}_{(\mathrm{aq})}+2 \mathrm{SiO}_{2}(\mathrm{~s})
$$

Step 2: (Conversion) $\mathrm{HCl}$ recovery by heating solution to $\sim 150{ }^{\circ} \mathrm{C}$. Hydrated magnesium chloride $\left(\mathrm{MgCl}_{2} \cdot 6 \mathrm{H}_{2} \mathrm{O}\right)$ is converted to $\mathrm{MgCl}(\mathrm{OH})$. Here the solution is heated to recover acid for the purposes of re-use in the first step. $\mathrm{MgCl}_{2} \cdot 6 \mathrm{H}_{2} \mathrm{O}$ initially loses water resulting in $\mathrm{MgCl}_{2} \cdot \mathrm{H}_{2} \mathrm{O}$, and then eventually $\mathrm{HCl}$ separates instead of further water release (Lackner et al., 1995).

$$
\mathrm{MgCl}_{2} \cdot 6 \mathrm{H}_{2} \mathrm{O}_{(\mathrm{aq})} \rightarrow \mathrm{MgCl}(\mathrm{OH})_{(\mathrm{s})}+\mathrm{HCl}_{(\mathrm{aq})}+5 \mathrm{H}_{2} \mathrm{O}_{(\mathrm{l})}
$$

Step 3: (Repartition) Water introduced to convert $\operatorname{MgCl}(\mathrm{OH})$ to $\mathrm{Mg}(\mathrm{OH})_{2}$. Through the introduction of water $\mathrm{MgCl}(\mathrm{OH})$ is converted into solid $\mathrm{Mg}(\mathrm{OH})_{2}$ and $\mathrm{MgCl}_{2}$ solution. These are then separated and $\mathrm{Mg}(\mathrm{OH})_{2}$ is sent for carbonation (Lackner et al., 1995).

$$
2 \mathrm{MgCl}(\mathrm{OH})_{(\mathrm{s})} \rightarrow \mathrm{Mg}(\mathrm{OH})_{2(\mathrm{~s})}+\mathrm{MgCl}_{2(\mathrm{aq})}
$$

Step 4: Magnesium hydroxide is subsequently carbonated at $407^{\circ} \mathrm{C}$ in an atmosphere of carbon dioxide at 1 bar.

$$
\mathrm{Mg}(\mathrm{OH})_{2(\mathrm{~s})}+\mathrm{CO}_{2(\mathrm{~g})} \rightarrow \mathrm{MgCO}_{3(\mathrm{~s})}+\mathrm{H}_{2} \mathrm{O}_{(\mathrm{g})}
$$


The relative free energy changes at each major process stage are presented in Figure 2-16. This variation, due to the formation of intermediate products, has been cited as a drawback, along with the energy required for the $\mathrm{HCl}$ recovery step (Teir, 2008). A study conducted by Newall et al. (2000) suggested this requirement could lead to four times the amount of $\mathrm{CO}_{2}$ produced to generate this energy compared to that sequestered by the process.

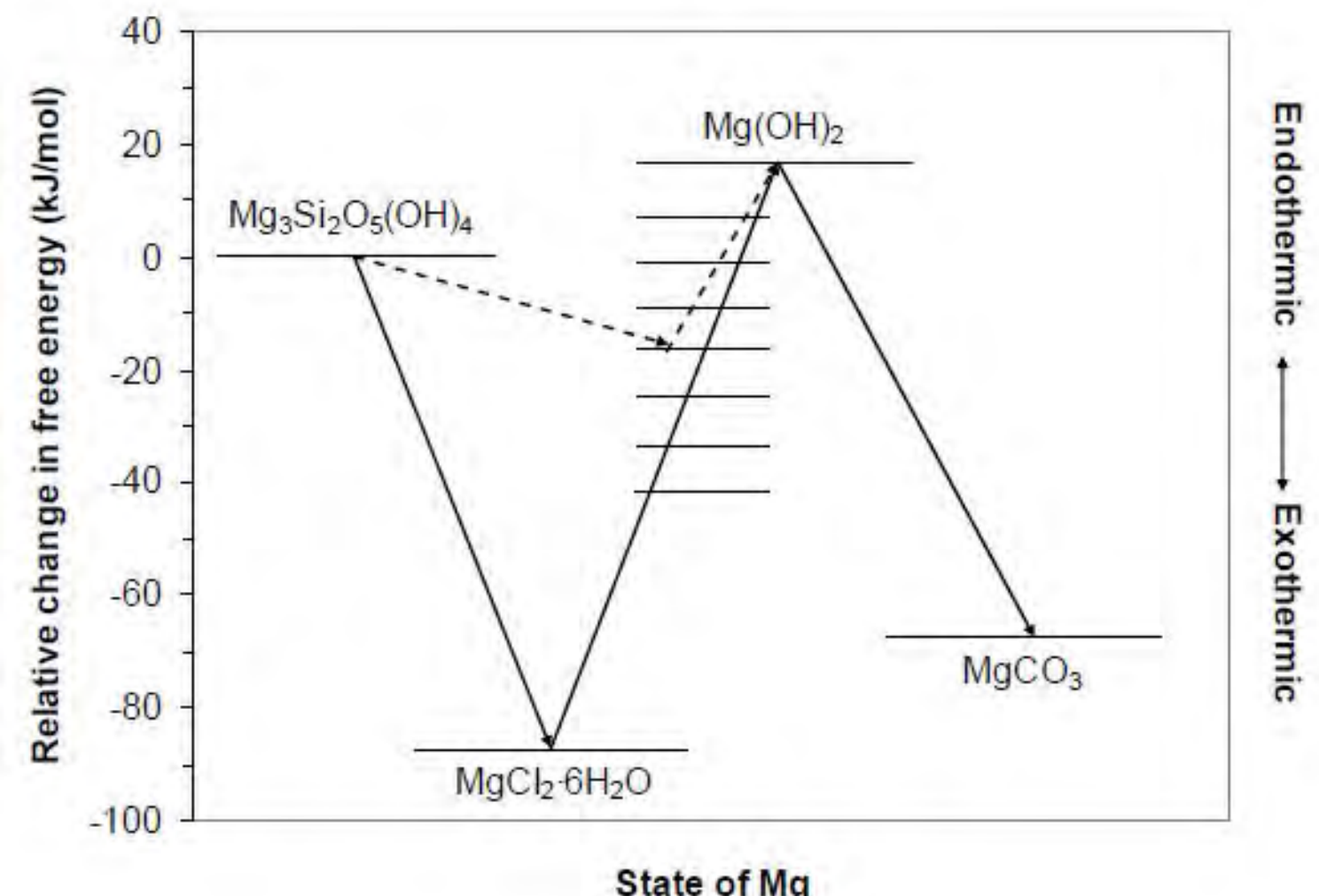

Figure 2-16: Relative free energy changes at the main process stages of the indirect carbonation (Teir, 2008)

To circumvent the high energy demand of the $\mathrm{HCl}$ recovery step, the use of molten melts of magnesium chloride was put forward by Wendt et al. (1998). This minimizes the energy requirement by reducing the amount of water due to application of a melt instead of hydrochloric acid for magnesium extraction. Optimum conditions for the carbonation of the silicate mineral with the melt were found to be $300{ }^{\circ} \mathrm{C}$ and 30 atm $\mathrm{P}_{\mathrm{CO}_{2}}$. This process would require substantial quantities of magnesium chloride which could account for a significant portion of global demand and upset the $\mathrm{CO}_{2}$ balance for the process (Newall et al., 2000).

Maroto-Valer et al. (2005) also conducted experiments that explored the activation of the silicate mineral, serpentine, through chemical and physical means to increase the surface area, thus improve the reaction rate. This approach is similar to Lackner et al. (1995) in that the silicate mineral is converted to magnesium hydroxide, albeit using different reagents. The 
authors used sulphuric acid to extract magnesium from serpentine producing magnesium sulphate at $50{ }^{\circ} \mathrm{C}$. This was then converted to magnesium hydroxide through the use of sodium hydroxide. The resulting magnesium hydroxide was carbonated at $20{ }^{\circ} \mathrm{C}$ and $45 \mathrm{~atm} \mathrm{CO}_{2}$ pressure. It was reported that a carbonation efficiency of $52.5 \%$ for magnesium hydroxide was obtained (Maroto-Valer et al., 2005). These conditions are considerably milder than those used other process routes like the direct aqueous route $\left(155^{\circ} \mathrm{C}, 115 \mathrm{~atm}\right)$. However, the chemical make-up and energy intensive regeneration are notable concerns that could negate the benefits of this process (Sanna et al., 2014b).

\section{Acetic Acid Process}

This route has the advantage of using a less corrosive medium in comparison to strong acids like hydrochloric acid and sulphuric acid (Rackley, 2010). The process, proposed by Kakizawa et al. (2001) separates the carbonation process into two process steps. Based on the experimental data obtained by the authors, they developed the conceptual flowsheet of the process presented in Figure 2-17:

Step 1: Dissolution: Acetic acid solution (13.72g acid/50g water) is used to extract calcium from wollastonite, at $60{ }^{\circ} \mathrm{C}$ and atmospheric conditions, producing calcium acetate and a silica gel that can be separated through a solid-liquid separation unit.

$$
\mathrm{CaSiO}_{3(\mathrm{~s})}+2 \mathrm{CH}_{3} \mathrm{COOH}_{(\mathrm{l})} \rightarrow \mathrm{Ca}^{2+}{ }_{(\mathrm{aq})}+2 \mathrm{CH}_{3} \mathrm{COO}_{(\mathrm{aq})}^{-}+\mathrm{H}_{2} \mathrm{O}_{(\mathrm{l})}+\mathrm{SiO}_{2}(\mathrm{~s})
$$

Step 2: Deposition and Crystallization: In this step, through the introduction of gaseous carbon dioxide at the reaction pressure of 30 bar into the calcium acetate solution, a calcium carbonate precipitate is produced at $60^{\circ} \mathrm{C}$. This step also recovers the acetic acid used in step 1:

$$
\mathrm{Ca}^{2+}{ }_{(\mathrm{aq})}+2 \mathrm{CH}_{3} \mathrm{COO}_{(\mathrm{aq})}^{-}+\mathrm{H}_{2} \mathrm{O}_{(\mathrm{l})}+\mathrm{CO}_{2(\mathrm{~g})} \rightarrow \mathrm{CaCO}_{3(\mathrm{~s})}+2 \mathrm{CH}_{3} \mathrm{COOH}_{(\mathrm{l})}
$$

The inventors argue that the use of acetic acid was selected on the basis that it is stronger than silicic acid but weaker than carbonic acid, which enables the carbonate to displace the acetate during the carbonation step of the process. Using a pulverised wollastonite sample, $48 \%$ extraction of calcium was reported in 250 minutes at $60{ }^{\circ} \mathrm{C}$ and atmospheric pressure. The extent of carbonation of calcium acetate was considerably less with a maximum of $20 \%$ carbonation reported at 30 bar and $60{ }^{\circ} \mathrm{C}$ (Kakizawa et al., 2001). 


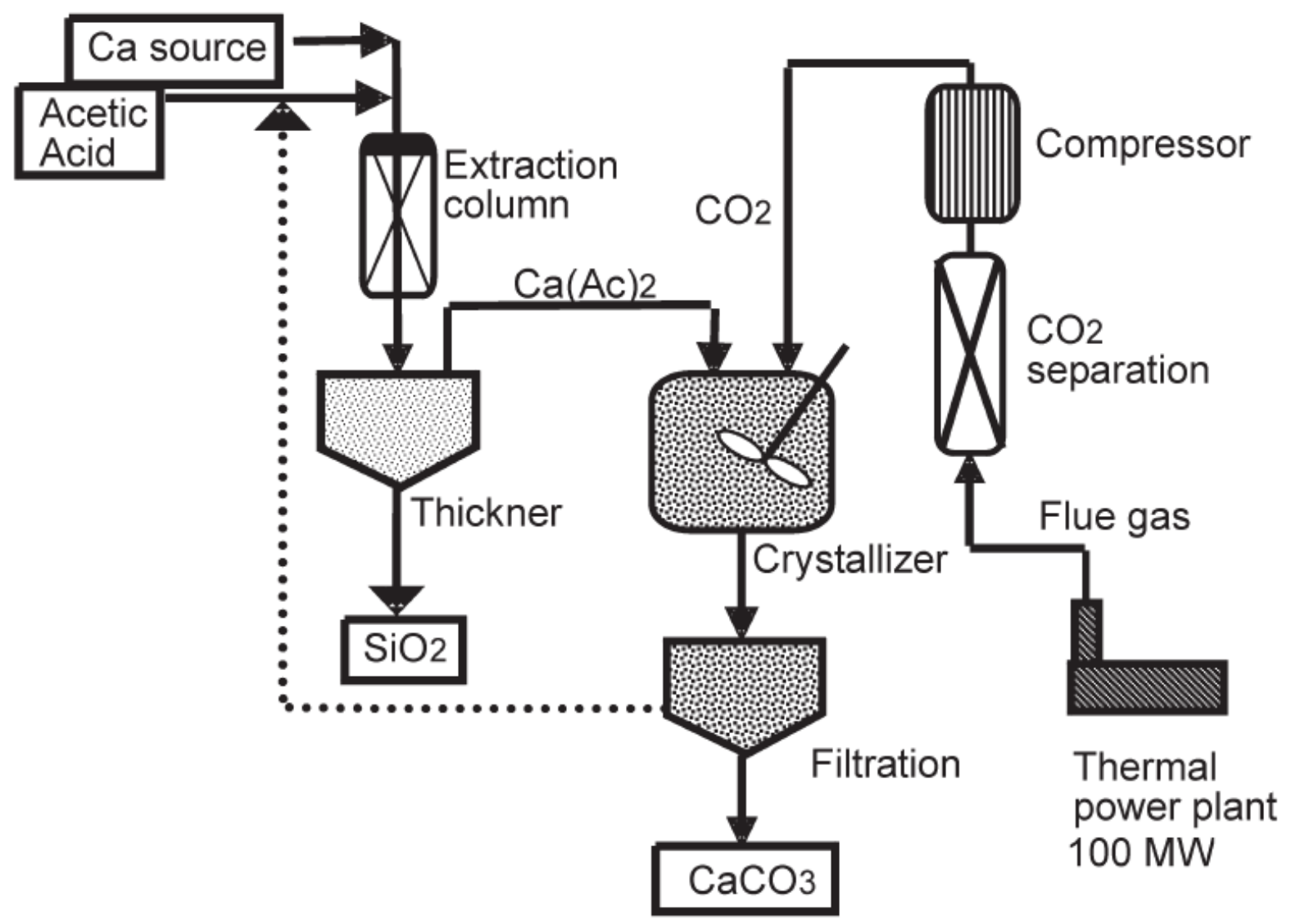

Figure 2-17: Schematic of acetic acid based carbonation of wollastonite (Kakizawa et al., 2001)

The use of this process for the carbonation of more reactive feedstocks such as wollastonite and calcium-rich waste streams such as steel slags (Eloneva et al., 2007) is attractive. It has been suggested that the carbonate product can be used to produce precipitated calcium carbonate (PCC) for the paper industry (Teir et al., 2005). This implies that a product stream of this process can be a useful feedstock to the paper industry, thus eliminating materials handling issues and generating revenues at the same time. Though the use of a less corrosive dissolution presents an attractive process route, the cost-effectiveness of this route is unclear. Furthermore, some researchers have raised concerns as to whether the recycling of acetic acid is feasible without excessive use of energy (Huijgen and Comans, 2003).

\section{Alkali Leach Process}

Blencoe et al. (2004) proposed an alkaline-based extraction of the reactive components from the silicate mineral. The process makes use of a concentrated solution of sodium hydroxide to extract $(\mathrm{Ca} / \mathrm{Mg})$-ions at high temperatures and pressure $\left(200^{\circ} \mathrm{C},<15 \mathrm{~atm}\right)$ producing reactive hydroxide compounds. Alongside the hydroxide, sodium silicate is produced which the inventors propose can be used to capture carbon dioxide forming sodium carbonate or sodium bicarbonate. These can then be reacted with magnesium hydroxide to produce magnesite and 
regenerate the reagent sodium hydroxide (Blencoe et al., 2004). Using olivine as an example, the main reactions for the process are presented:

a. Alkali Leach

$$
\mathrm{Mg}_{2} \mathrm{SiO}_{4}+2 \mathrm{NaOH}+\mathrm{H}_{2} \mathrm{O} \rightarrow 2 \mathrm{Mg}(\mathrm{OH})_{2}+\mathrm{Na}_{2} \mathrm{SiO}_{3}
$$

b. $\mathrm{CO}_{2}$ Capture

$$
\mathrm{Na}_{2} \mathrm{SiO}_{3}+2 \mathrm{CO}_{2}+\mathrm{H}_{2} \mathrm{O} \rightarrow 2 \mathrm{NaHCO}_{3}+\mathrm{SiO}_{2}
$$

c. Carbonation

$$
\mathrm{Mg}(\mathrm{OH})_{2}+\mathrm{NaHCO}_{3} \rightarrow \mathrm{MgCO}_{3}+\mathrm{NaOH}+\mathrm{H}_{2} \mathrm{O}
$$

A number of potential disadvantages for this process have been highlighted (IPCC, 2005; Huijgen, 2007; Wang, 2011). Firstly relatively long reaction times are required (up to 3days) to obtain reasonable recoveries. Secondly, the reaction is reported to require relatively fine particle size (10 microns) which would require extensive grinding. In addition, the ability to recycle the sodium hydroxide solution has yet to be proven. If this is not effective, large quantities of sodium hydroxide required.

\subsection{Environmental Assessment}

There is a need for the development of technically feasible, carbon-neutral mineral carbonation processes in order to reduce global $\mathrm{CO}_{2}$ emissions. Though the majority of research and development has focused on the enhancement of extraction and carbonation efficiencies, there is a growing recognition of the need to develop integrated mineral carbonation processes that reduce input requirements in terms of energy and reagents.

A feasibility study conducted by Newall et al. (2000) investigated a number of processes that included the route proposed by Lackner et al. (1995) for magnesium and calcium feedstocks, a direct carbonation approach and an indirect approach that uses a magnesium melt to bypass the use of hydrochloric acid, as is the case in the route proposed by Lackner et al. (1995). A table summarising the processes selected from initial screening of literature by the authors is presented in Table 2-6. Newall et al (2000) narrowed down the selection, through further screening, with the view of selecting one process as "favoured" to be implemented as a sequestration option. To achieve this, "key decision factors" were selected to compare the processes: efficiency of reaction, cost, confidence in process, acceptance and overall applicability. 
Table 2-6: Selected carbonation processes from literature (Newall et al., 2000)

\begin{tabular}{|c|c|c|c|c|}
\hline $\begin{array}{c}\text { Process } \\
\text { No. }\end{array}$ & Raw Material & $\begin{array}{c}\text { Raw material } \\
\text { reacts with: }\end{array}$ & $\begin{array}{c}\mathbf{C O}_{2} \text { reacts } \\
\text { with: }\end{array}$ & $\begin{array}{c}\mathbf{C O}_{2} \text { stored } \\
\text { as: }\end{array}$ \\
\hline 1 & $\mathrm{Mg}$ silicate rock & $\mathrm{HCl}$ & $\mathrm{Mg}(\mathrm{OH})_{2}$ & $\mathrm{MgCO}_{3}$ \\
\hline 2 & $\mathrm{Mg}$-rich brine deposit & $\mathrm{H}_{2} \mathrm{O}$ & $\mathrm{Mg}(\mathrm{OH})_{2}$ & $\mathrm{MgCO}_{3}$ \\
\hline $3 \mathrm{a}$ & $\mathrm{Mg}$ silicate rock & $\mathrm{MgCl}_{2}$ & $\mathrm{Mg}(\mathrm{OH})_{2}$ & $\mathrm{MgCO}_{3}$ \\
\hline $3 \mathrm{~b}$ & $\mathrm{Mg}$ silicate rock & $\mathrm{CO}_{2}$ & $\mathrm{Mg}_{3} \mathrm{Si}_{2} \mathrm{O}_{5}(\mathrm{OH})_{4}$ & $\mathrm{MgCO}_{3}$ \\
\hline 4 & $\mathrm{Ca}$ silicate rock & $\mathrm{HCl}$ & $\mathrm{Ca}(\mathrm{OH}) 2$ & $\mathrm{CaCO}_{3}$ \\
\hline 5 & $\mathrm{MgCO}_{3}+\mathrm{NaCl}$ & $\mathrm{CO}_{2}$ & $\mathrm{MgCO}+\mathrm{NaCl}$ & $\mathrm{NaHCO}_{3}$ \\
\hline
\end{tabular}

The study was based on a thermodynamic view of the processes to assess them due to the absence of experimental data on kinetics (Newall et al., 2000). Literature data and approximations of process unit mass and energy requirements were used, as the kinetics of the reactions were not known.

The authors studied two processes in detail: the $\mathrm{HCl}$ extraction route proposed by Lackner, as well as the carbonation of the silicate mineral in a melt of $\mathrm{MgCl}_{2}$ (Wendt et al., 1998). These processes were to be developed to sequester 3 million tonnes of $\mathrm{CO}_{2}$ per year produced by a 500 MWe coal-based power station. Literature data was used to estimate the $\mathrm{CO}_{2}$ balance of the process, considering quarrying and crushing of the mineral, processing (heating and electricity) as well as waste disposal. The results of this study found that the process proposed by Lackner et al. (1995) resulted in $13.9 \mathrm{Mt} /$ annum $\mathrm{CO}_{2}$ emissions for the sequestration of 3 Mt/annum carbon dioxide, with the dehydration step accounting for the majority of the emissions. In contrast, the carbonation process using the magnesium chloride melt resulted in only $0.825 \mathrm{Mt} /$ annum $\mathrm{CO}_{2}$ emissions, amounting to $27 \%$ of the carbon dioxide sequestered. However, it should be noted that the carbonation of the silicate mineral in a melt required considerable quantities of make-up magnesium chloride, the impact on the carbon footprint of which was not considered. Considering that the generation of the melt on-site has been estimated to account for a third of annual global $\mathrm{HCl}$ production (O'Connor et al., 2005), this may significantly underestimate the potential environmental impact of the magnesium melt approach.

To evaluate the potential of the direct aqueous carbonation process (Section 2.1.2), developed by the National Energy Technology Laboratory - NETL (formerly the Albany Research Center), a study was conducted to establish the energy burdens of this route (O'Connor et al., 
2005 ) at the optimum mineral specific conditions discussed in Section 2.1.2. The samples used were obtained from seven different regions, with Region 1 and 5 corresponding to olivine, Region 2-4 and Region 6 corresponding to serpentine, and Region 7 to wollastonite. Energy requirements for feedstock preparation and pre-treatment were included in the study, although the energy requirements for reagent production do not appear to have been accounted for. The results are summarised in Table 2-7. This study attributed most of the energy burdens of this approach to pre-treatment techniques such as standard crushing and grinding for all minerals, additional heat treatment of serpentine and mechanical activation through ultrafine grinding of wollastonite and olivine.

Table 2-7: Energy burdens for the NETL mineral carbonation route $\left(O^{\prime}\right.$ Connor et al., 2005)

\begin{tabular}{|c|c|c|c|c|c|c|c|}
\hline \multirow[b]{2}{*}{ Region } & \multirow{2}{*}{$\begin{array}{c}\text { Energy, } \\
\text { GW } \cdot \mathbf{h} \\
(\mathbf{x 1 0 0 0})\end{array}$} & \multirow{2}{*}{$\begin{array}{c}\mathrm{CO}_{2} \\
\text { seq., } \mathrm{Mt}^{1}\end{array}$} & \multicolumn{3}{|c|}{$\begin{array}{c}\text { Energy Consumption, } \\
\text { GW } \cdot h / M t \text { CO2 seq. }\end{array}$} & \multicolumn{2}{|c|}{$\begin{array}{c}\mathrm{CO}_{2} \text { avoided, } \\
\mathrm{Mt}\end{array}$} \\
\hline & & & std. $^{2}$ & act. $^{3}$ & Total & std & act \\
\hline 1 & 18 & 18 & 300 & 333 & 633 & 13 & 7 \\
\hline 2 & 9 & 10 & 180 & 2022 & 2202 & 8 & 0 \\
\hline 3 & 9 & 10 & 180 & 2251 & 2431 & 8 & 0 \\
\hline 4 & 72 & 72 & 180 & 2022 & 2202 & 59 & 0 \\
\hline 5 & 184 & 187 & 320 & 333 & 653 & 126 & 63 \\
\hline 6 & 220 & 231 & 180 & 829 & 1009 & 187 & 0 \\
\hline 7 & 75 & 76 & 190 & 239 & 429 & 62 & 43 \\
\hline
\end{tabular}

${ }^{1} \mathrm{CO}_{2}$ sequestered based on coal consumption and carbon content by region, assumes sequestration of $100 \%$ of emissions

${ }^{2}$ Energy consumption for complete sequestration operation, including energy for standard pretreatment (crushing and grinding) and carbonation energy

${ }^{3}$ Energy for activated pretreatment, ultrafine grinding (olivine and wollastonite) or thermal (serpentine)

Table 2-7 indicates that for the regions $2-4$, as well as region 6 there is zero carbon dioxide avoided due to activation through activation pretreatment. These cases correspond to the use of serpentine as feedstock, which, in addition to crushing and grinding, requires high temperature thermal treatment to improve its reactivity. Significant decreases in carbon dioxide avoided can also be noted for the other regions which correspond to olivine and wollastonite, when ultrafine grinding is used to improve reactivity. These results indicate that serpentinebased direct aqueous carbonation processes are unlikely to be viable for sequestration purposes. Similar findings were presented by Khoo and Tan (2006) who explored carbonation processes in isolation as well as coupled with carbon dioxide capture. However, the study by O'Connor 
et al. (2005) appeared to ignore the impact of chemical reagents, which would result in lower carbon dioxide avoided than those reported in Table 2-7, when their impacts were accounted for. Additionally, the study appeared to combine the energy from heat and electricity in their analysis, which may cause difficulty in accounting for carbon dioxide emissions attributed to each energy source.

A gate-to-gate study was conducted to investigate the viability of different routes to sequester carbon dioxide from a $1 \mathrm{GW}$ coal-based power plant, by Kelly et al. (2011). The options considered were the carbonation of brine solutions through the use of industrial caustics such as sodium hydroxide, the direct aqueous carbonation of silicate minerals, as well as the direct aqueous carbonation of industrial wastes such as iron slag and fly ash. The analysis was based on publicly available literature data on these approaches, and rough engineering calculations to estimate energy requirements. Their energy analysis included carbon dioxide capture and major process unit operations such as compressors, mixers and heaters (Kelly et al., 2011). The silicate minerals selected were olivine and wollastonite, the authors arguing these are the most promising feedstocks. The evaluation of process performance was conducted by comparing energy required by the process versus the energy generated by the $1 \mathrm{GW}$ power plant. The use of industrial wastes in brine solutions was reported to result in an energy penalty of $90-100+\%$ of the energy generated. Amongst the silicate minerals, olivine was reported to result in a 55$69 \%$, whereas wollastonite resulted in an energy penalty greater than $100 \%$. The industrial waste, fly ash, reported the least energy penalty (9-22\%), whereas iron slag had an energy penalty greater than $100 \%$ (Kelly et al., 2011). The separation of carbon dioxide from flue gases and the grinding of the minerals were found to be the energy major burdens, for the olivine, wollastonite and iron slag feedstock, whereas the production of sodium hydroxide was the main contributor to the energy penalty for the brine solutions route. To make the processes feasible Kelly et al. (2011) suggested optimisation of the process to enhance energy efficiency, though opportunities for this were not explicitly identified.

Kirchofer et al. (2012) conducted a life cycle assessment of the direct aqueous carbonation process using different mineral feedstock in order to compare their energy efficiency and carbon dioxide storage potential. The approach the authors took relied upon literature data, patents, assumptions and simple engineering calculations to evaluate mass and energy balances. They used an economic input-output life cycle assessment (EIO-LCA) tool developed at Carnegie Mellon University to convert direct energy consumption to life cycle energy consumption, which includes on-site as well as input requirements. The study by 
Kirchofer et al. (2012) considered a variety of mineral carbonation feedstock from natural silicates such as serpentine (Se) and olivine (Ol) to industrial wastes such as steel slags (SS), coal fly ash (FA) and cement kiln dust (CKD). The model used included eight stages across the life cycle of the process from extraction, to chemical conversion to disposal. However, the researchers did not include energy requirements associated with chemical reagent production and the compression of carbon dioxide. Different conditions were investigated with regards to temperature, from $25^{\circ} \mathrm{C}$ up to as high as $155^{\circ} \mathrm{C}$. The authors reported that heating and physical pre-processing (crushing and grinding) were major carbon dioxide emissions impacts for the natural silicate minerals as indicated in Figure 2-18.

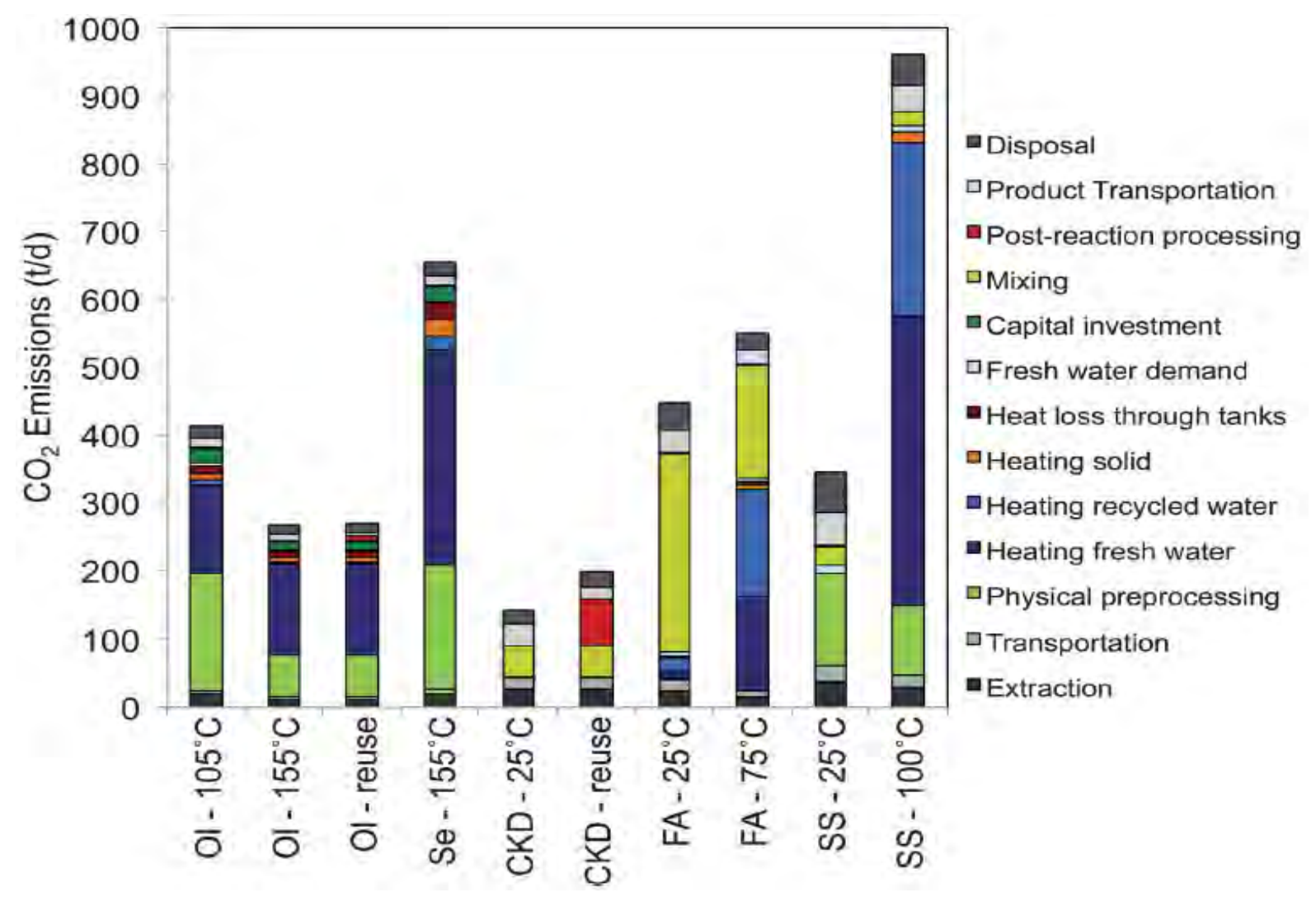

Figure 2-18: $\mathrm{CO}_{2}$ emissions for the direct aqueous mineral carbonation processes as a function of feedstock and carbonation (Kirchofer et al., 2012)

Regardless, the total carbon dioxide emissions from the processes considered were less than the 1000 ton/day sequestered for all the sources examined. Though this result is encouraging, it is important to note that the model used by the researchers was a general, first-order level model, which could potentially underestimate some contributions. It was duly noted that more detailed process models developed on software like Aspen could provide better estimates for reaction rates and energy requirements (Kirchofer et al., 2012). 
The life cycle assessment of the multi-stage process developed by researchers at the Ábo Akademi University (Fagerlund et al., 2009; Zevenhoven et al., 2011) was conducted by another researcher in this group (Ndaugu et al., 2012b). The researchers' work compared the $\AA A U$ process to the direct aqueous carbonation process developed at the NETL. These processes were discussed in detail in Sections 2.1.1 and 2.1.2, respectively. To determine energy requirements, Nduagu et al. (2012b) used literature data for the aqueous carbonation process and developed a simulation on Aspen software for the multi-stage process. The source of carbon dioxide was considered to be a coal-fired power plant based on Canadian/US data from the Ecoinvent database on SimaPro software. The plant was assumed to supply the electricity requirements whereas natural gas was considered for heat supply (Nduagu et al., $2012 b$ ). However, the authors did not account for reagent make-up (assuming 100\% recovery) for the ammonium sulphate used in the ÅAU process. Additionally, the determination of the amount of reagent $\left(\mathrm{NaHCO}_{3}, \mathrm{NaCl}\right)$ for the NETL process was not based on the material balance (Nduagu et al., 2012b), which underestimates the amounts required and thus the impact in terms of emissions, according to the authors.

The authors used systems expansion through the use of iron and calcium by-products in the iron steel making industry, mass allocation by products, as well as heat integration to reduce environmental burdens of the process by as much as $35 \%$ for the multi-stage process. Through the application of an exergy analysis the authors reported significantly lower environmental impacts $\left(683 \mathrm{~kg}-\mathrm{CO}_{2} \mathrm{e} / \mathrm{ton}-\mathrm{CO}_{2}\right)$ in comparison to $1270-2170 \mathrm{~kg}-\mathrm{CO}_{2} \mathrm{e} / \mathrm{ton}-\mathrm{CO}_{2}$ when an exergy analysis was not conducted for the NETL process (Nduagu et al., 2012b). The study found that both processes were effective carbon sinks with the multi-stage process having an environmental burden of $517 \mathrm{~kg}-\mathrm{CO}_{2} \mathrm{e} /$ ton- $\mathrm{CO}_{2}$ (Nduagu et al., 2012b). This signifies promise for these processes though it must be noted that the impact of potential fresh ammonium sulphate use in the multi-stage process to environmental burdens was not discussed.

A comparison of the $\mathrm{CO}_{2}$ equivalents sequestered by the NETL direct aqueous carbonation and the ÅAU multi-stage process was also conducted in a more recent study by Giannoulakis et al. (2014). In comparison to the study by Nduagu et al. (2012b), this was a less rigorous evaluation, making use of simple engineering calculations to determine energy requirements. In this case the source of carbon dioxide was either a pulverized hard coal power plant or a natural gas combined cycle (NGCC) power plant in Europe, in the year 2025. The silicate minerals wollastonite, olivine and serpentine were considered for the NETL process and serpentine for the $\AA \mathrm{AU}$ process albeit with current achievable conversion (considered to be $75 \%$ extraction 
and $65 \%$ carbonation) as well as improved future conversion (considered to be $80 \%$ extraction and $90 \%$ carbonation). Three different scenarios are considered for the direct aqueous carbonation process based on feedstock (NETL1 - Wollastonite, NETL2 - Olivine, NETL3 Serpentine) and two for the $\AA A U$ process, based on extraction/carbonation efficiencies ( $\AA A U 1$ - current efficiency, ÅAU2 - future improved efficiency). The authors further compared these processes with geological storage. A summary of their findings with regards to carbon dioxide footprints is presented in Figure 2-19.

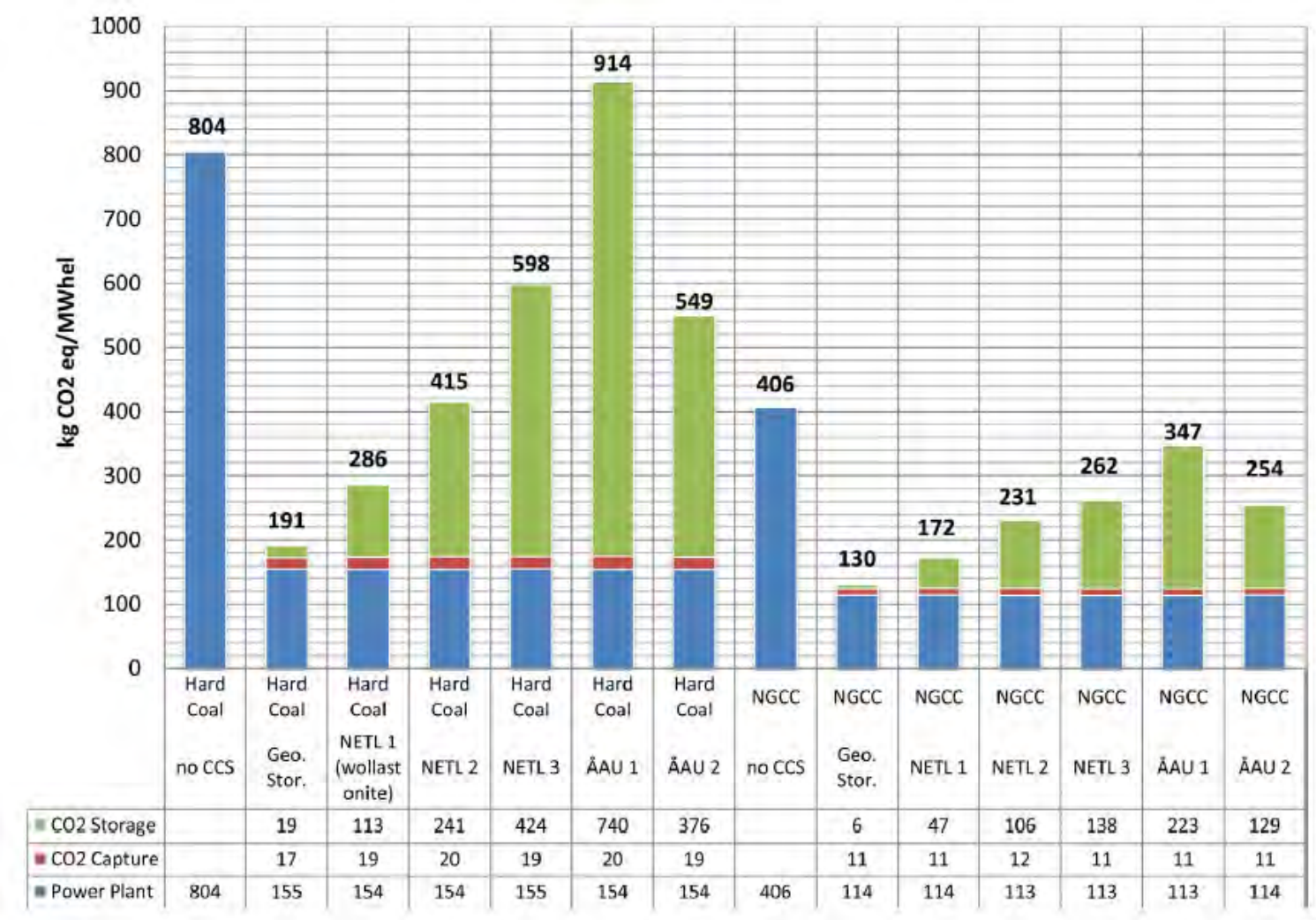

Figure 2-19: Life cycle greenhouse gas emissions for mineral carbonation and geological storage in Europe in 2025 (Giannoulakis et al., 2014)

In most cases, the sequestration of carbon dioxide resulted in a reduction in net emissions from the base case amount of $804 \mathrm{~kg}-\mathrm{CO}_{2} \mathrm{e}$. An exception was the $\AA \mathrm{AU} 1$ for hard coal power plant, based on current conversion efficiencies of $75 \%$ extraction and $65 \%$ carbonation reported by Romao et al. (2012). The major burdens were attributed to solvent make-up, heat and electricity requirements (Giannoulakis et al., 2014). However, for chemical reagent accounting for the NETL process the authors referenced the work by Nduagu et al. (2012b) which underestimates the required input reagents. Additionally, the authors assumed the heat required for the heating of process streams in the NETL process to be balanced by the released by the carbonation reaction, in effect complete heat integration. Although this is a reasonable simplification, the 
recovery of the heat from the carbonation reaction may be difficult, as some researchers have noted (Zevenhoven et al. 2011; Olajire, 2013).

\subsection{Literature Summary}

\subsubsection{General Observations}

Mineral carbonation has the potential to play a key role in the sequestration of anthropogenic carbon dioxide emissions. The development of this process has spanned more than three decades and has resulted in the development of an array of process routes and processes, all with the objective to permanently store carbon dioxide in stable carbonate compounds. However, considerable challenges stand between the technology and effective sequestration of carbon dioxide, from an environmental and economic stand point.

The direct carbonation of the silicate mineral with carbon dioxide, the simplest approach, and one that offers the most potential for the use of the exothermic energy of the carbonation reaction, has been demonstrated to be prohibitively slow and is thus not suitable for industrial implementation. As a result, the general trends have been towards the development of multistage gas/solid carbonation processes or the use of aqueous media to accelerate the process. Current carbonation of silicate minerals in engineered carbonation systems generally requires relatively aggressive, energy-intensive processes and/or pretreatment to enhance the reaction kinetics. Researchers at the Åbo Akademi University ( $\AA \mathrm{AU})$ have focused on developing a high temperature multi-stage gas/solid route (Section 2.1.1), whilst the majority of other researchers in this field have been focused on developing aqueous-based carbonation systems (Section 2.1.2 and 2.1.3). In this area, the direct aqueous process developed at the National Energy Technology Laboratory (NETL) has been considered the state-of-the-art. Alternatively, others have pursued indirect aqueous processes such as the multi-stage hydrochloric acid extraction, acetic acid and the alkali leach processes. However, the $\mathrm{pH}$ swing method has largely been the most adopted. Several of these have been developed into conceptual flowsheets and some patented, such as the ammonium salts process, with potential to be implemented on an industrial scale due to the reaction times and conversion efficiencies.

Studies on mineral carbonation have mainly focused on the more abundant and reactive silicate minerals wollastonite, serpentine and olivine. Relatively few studies have been conducted using less reactive pyroxene minerals, which are the major source of sequestrable cations in the tailings generated from the processing of PGM ores in South Africa. By extension the processes that have been developed have focused on these minerals, with no known process 
having been proposed and evaluated using PGM tailings as a feedstock. The fact that limited research has been done using pyroxene or PGM tailings implies that the understanding of the opportunities that exist in particular process routes is minimal, and that technical operability of the processes beyond the specific mineral is uncertain. This clearly presents a challenge with regards to process selection. Regardless, the selection of process routes can be made, through good engineering judgement, by analysing process thermodynamics and reactivity, keeping in mind the type of feedstock. Review papers give a good indication of the state-of-the-art and thus serve as an additional guide.

Despite the potential advantages of using these tailings as feedstock for a uniquely South African engineered mineral carbonation process, preliminary studies have indicated that relatively aggressive processing conditions and/or long retention times will be required to make this technically feasible. The consequence of using aggressive reaction conditions to accelerate the mineral carbonation process has been the potential to render this approach an ineffective carbon sink, from a net carbon footprint perspective. Recent studies are consistent with an increasing recognition of the need to evaluate the processes using life-cycle approaches to ensure that they result in net $\mathrm{CO}_{2}$ reduction. However, to date studies have been based largely on rough calculations, simplifying assumptions, and limited use of LCA tools and robust simulation software packages that contain appropriate thermodynamic models to better predict material and energy requirements (Section 2.2). Although the importance of utilising more rigorous mass and energy balance tools such as Aspen Plus has been acknowledged, application has been limited. Additionally, the accounting of carbon dioxide footprints has also been largely conducted on the basis of first-order calculations, rather than by means of a rigorous software framework which takes into account all the relevant processes, including the foreground and background processes. This has led to a lack of consistency and clarity in approach, difficulty in drawing comparisons, and ultimately a compromise of the veracity of the studies.

\subsubsection{Summary and Comparison of Mineral Carbonation Processes}

The literature review indicated that several process routes exist for the mineral carbonation of silicate minerals. It also demonstrated that within these routes there has been a considerable amount of research that has been done to understand and improve the performance of the processes. Despite this, only a few of these processes have been developed into pilot or commercial projects, with only a direct aqueous based process developed by Shell having been demonstrated on a pilot scale, whereas the brine solutions based SkyMine process (by a joint 
venture that includes $\mathrm{BP}$ and Conoco-Phillips) has been commercially implemented to sequester $83000 \mathrm{tCO}_{2}$ per annum (Sanna et al., 2014b).

The direct gas-solid carbonation route has been developed from the simple single-step process to a more complex multi-step indirect process. This indirect route has achieved notably higher conversions and better reactivity through the use of magnesium hydroxide as the carbonation feedstock. The ÅAU process, which uses ammonium sulphate to extract magnesium from serpentine, and subsequently producing magnesium hydroxide to be carbonated, offers promising levels of extraction. Typical extraction efficiencies have been reported to be around $64-66 \%$ whereas carbonation efficiencies have been reported to reach a maximum of $55 \%$ (Nduagu et al, 2012a; Nduagu, 2012). Some have even set future targets of conversion up to as high as 90\% (Romao et al, 2012). However, this process uses energy intensive conditions for the extraction stage, with temperatures as high as $400{ }^{\circ} \mathrm{C}$. The researchers have claimed that their approach allows for the recovery of energy released by the carbonation reaction (Zevenhoven et al., 2011; Nduagu et al., 2012b), which occurs at around $450^{\circ} \mathrm{C}$ and 20 bar. A literature review on life-cycle assessment studies of this process has provided conflicting findings with one study suggesting that process could result in a net release of carbon dioxide (Giannoulakis et al., 2014) whereas another conducted by researchers at the ÅAU suggested the process could result in net sequestration of carbon dioxide through the use of mass allocation, systems expansion and energy integration (Nduagu et al., 2012b). These were not considered in the study by Giannoulakis et al. (2014), who used simpler first-order approximations of energy and carbon footprints. Despite the use of high temperature and pressure conditions, this process is promising due to the recycling and possible energy integration opportunities. The application of this process to silicate mineral feedstock other than serpentine has not been discussed in the literature.

The direct aqueous mineral carbonation process, developed at the NETL, has been reported to provide relatively high conversion for the major silicate minerals (Gerdemann et al., 2007), and can also be considered a simpler carbonation processes. The development of this process has evolved from the use of distilled water, to the addition of chemical reagents like sodium chloride and sodium bicarbonate. Laboratory testwork conducted on serpentine, olivine and wollastonite indicate that the optimum conditions vary according to the silicate mineral, but generally require high pressure $\left(>40\right.$ atm $\left.\mathrm{P}_{\mathrm{CO}_{2}}\right)$ and temperature $\left(>100{ }^{\circ} \mathrm{C}\right)$. The overall carbonation efficiency of these minerals has been reported to be more than $50 \%$ for the least reactive mineral, serpentine, and up to $80 \%$ for the most reactive mineral, olivine, under the 
optimum conditions (O'Connor et al., 2005; Gerdemann et al., 2007). The pre-requisite high temperature pretreatment of serpentine and grinding of the silicate mineral to particle sizes below 75 microns results in prohibitively high energy requirements (O'Connor et al., 2005). Hence, avoiding the need for heat treatment appears to be key to the viability of serpentine as a feedstock and might justify the use of chemical activation, through reagent use, to extract the alkaline metals as an alternative (Park and Fan, 2004; Teir et al., 2007a). The process has also been evaluated from a life cycle assessment perspective (Nduagu et al., 2012b; Giannoulakis et al. 2014). Both studies have indicated that the direct aqueous carbonation process results in net sequestration of carbon dioxide for wollastonite, olivine and serpentine. However the authors appear to not have adequately accounted for chemical reagent make-up and assuming recoverability of carbonation heat of reaction. This is unlikely to be achievable in practise. Nevertheless, the preliminary results are promising and the simplicity of the process makes it attractive.

Indirect aqueous processes have been noted to offer opportunities for individual optimisation of process steps. These processes involve a series of at least two process steps prior to carbonation of the metal ions extracted from the silicate mineral matrix. Several indirect aqueous mineral carbonation processes have been discussed in this review. Of the indirect aqueous mineral carbonation processes, the $\mathrm{pH}$ swing process has been the subject of the most research (Park et al., 2003; Park and Fan, 2004; Teir et al., 2007a; Wang and Maroto-Valer, 2013; Meyer et al., 2014). The review has shown that various acidic chemical reagents can be used for extraction, including inorganic acids, organic acids, ammonium salts or even a mixture of these. The adjustment of the $\mathrm{pH}$ can be achieved through the use of various basic reagents such as sodium hydroxide and ammonium hydroxide. The ammonium salts process (Wang and Maroto-Valer, 2013), developed mainly for serpentine feedstocks, shows great promise through its integration of capture, regeneration and sequestration. This is a conceptual process, based on a process developed by Pundsack (1967), which has been patented in the United States by the authors (Wang and Maroto-Valer, 2013). The operating conditions have been reported to be $90{ }^{\circ} \mathrm{C}$ for extraction and $80{ }^{\circ} \mathrm{C}$ for carbonation. The researchers have reported extraction efficiencies of more than $60 \%$ and carbonation efficiencies of more than $90 \%$, for serpentine samples. On the other hand, using the same extraction reagent (ammonium bisulphate), Sanna et al. (2014a) has reported extraction efficiencies of 30\% for magnesium from pyroxene, a major component of PGM tailings. Despite the apparent advantages of the ammonium salts process, no detailed energy analysis and carbon footprint evaluation has been conducted to 
date. The high temperature used for evaporation of water prior to the high temperature thermal decomposition of ammonium sulphate is concerning, as highlighted by Dri et al. (2014).

As an alternative to this process, based on dissolution and carbonation studies conducted on pyroxene and PGM tailings (Vogeli et al., 2011; Meyer et al., 2014), a hydrochloric acid based pH swing method has been reported to attain extraction efficiencies of $20 \%$ and carbonation efficiencies up to $69 \%$ (Vogeli, 2012). This approach is the only reported route to have been used to conduct carbonation experiments with PGM tailings as a feedstock. It is worth noting that studies have been limited to preliminary laboratory scale tests, with no testwork on the recovery and regeneration of reagents. Similarly, no assessment of the carbon dioxide balance has been conducted.

Lackner's multi-stage hydrochloric acid extraction process, developed on the basis of serpentine (Lackner et al., 1995), is another example of indirect aqueous processes. This is a conceptual process developed without accompanying experimental data by the researchers. This process converts cations in the silicate mineral into more reactive magnesium hydroxide, and subsequent conversion of the more reactive hydroxide to carbonate. The potential to recycle hydrochloric acid during dehydration of magnesium chloride could reduce material requirements of fresh hydrochloric acid feed. Though this is an attractive element of this process, some authors have pointed out that this regeneration comes at the expense of substantial amounts of energy that threaten process sustainability (Newall et al, 2000). Another multi-stage, indirect aqueous process, the acetic acid process, has been shown to be only viable for reactive feedstocks such as wollastonite and industrial wastes (Kakizawa et al., 2001). The alkali leach process (Blencoe et al., 2004), which involves the use of sodium hydroxide as the leaching agent, has been reported to require long reaction times of up to 72 hours for the extraction stage, ultrafine grinding $(<10 \mu \mathrm{m})$ of the mineral, as well as potentially large quantities of reagent input due to unproven sodium hydroxide recycle (IPCC, 2005; Huijgen, 2007). Table 2-8 summarises the key attributes of selected mineral carbonation processes. 
Table 2-8: Summary of selected processes and their key attributes

\begin{tabular}{|c|c|c|c|c|c|c|}
\hline Selected Processes & $\begin{array}{c}\text { Mineral } \\
\text { Tested }\end{array}$ & $\begin{array}{l}\text { Chemical } \\
\text { Reagents }\end{array}$ & Temperature & Pressure & Conversion & References \\
\hline $\begin{array}{l}\text { Ammonium Salts } \\
\text { (Indirect pH Swing) }\end{array}$ & $\begin{array}{l}75-150 \mu \mathrm{m} \\
\text { Serpentine }\end{array}$ & $\begin{array}{c}1.4 \mathrm{M} \\
\mathrm{NH}_{4} \mathrm{HSO}_{4}\end{array}$ & $\begin{array}{c}90^{\circ} \mathrm{C}- \\
\text { extraction } \\
80^{\circ} \mathrm{C}- \\
\text { carbonation }\end{array}$ & atmospheric & $\begin{array}{l}>60 \% \text { extraction } \\
>90 \% \text { carbonation }\end{array}$ & $\begin{array}{c}\text { Wang and } \\
\text { Maroto-Valer, } \\
\text { 2013; Wang, } \\
2013\end{array}$ \\
\hline $\begin{array}{l}\text { Lackner's Multi-stage } \\
\text { (Indirect Multi-stage) }\end{array}$ & Serpentine & $\mathrm{HCl}$ & $\begin{array}{c}80{ }^{\circ} \mathrm{C}-\text { extraction } \\
407{ }^{\circ} \mathrm{C}- \\
\text { carbonation } \\
\end{array}$ & atmospheric & N/A & $\begin{array}{c}\text { Lackner et al., } \\
\text { 1995; Newall } \\
\text { et al., } 2000 \\
\end{array}$ \\
\hline $\begin{array}{c}\text { Åbo Akademi } \\
\text { University } \\
\text { (Indirect Multi-stage) }\end{array}$ & $\begin{array}{l}75-125 \mu \mathrm{m} \\
\text { Serpentine }\end{array}$ & $\left(\mathrm{NH}_{4}\right)_{2} \mathrm{SO}_{4}$ & $\begin{array}{l}400-440{ }^{\circ} \mathrm{C}- \\
\text { extraction } \\
450-500{ }^{\circ} \mathrm{C}- \\
\text { carbonation }\end{array}$ & $\begin{array}{l}\text { atmospheric- } \\
\text { extraction } \\
20 \text { bar - } \\
\text { carbonation }\end{array}$ & $\begin{array}{l}64-66 \%- \\
\text { extraction } \\
50-55 \%- \\
\text { carbonation }\end{array}$ & $\begin{array}{c}\text { Fagerlund et } \\
\text { al., 2010; } \\
\text { Nduagu et al., } \\
2012 \mathrm{a} / \mathrm{b} ; \\
\text { Nduagu, 2012 } \\
\end{array}$ \\
\hline $\begin{array}{c}\text { Mineral Acid pH } \\
\text { Swing } \\
\text { (Indirect pH Swing) }\end{array}$ & $\begin{array}{l}75-106 \mu \mathrm{m} \\
\text { PGM tailings }\end{array}$ & $\begin{array}{c}2 \mathrm{M} \mathrm{HCl} \\
15 \mathrm{M} \mathrm{NaOH}\end{array}$ & $\begin{array}{c}70^{\circ} \mathrm{C}- \\
\text { extraction } \\
20^{\circ} \mathrm{C}- \\
\text { carbonation }\end{array}$ & atmospheric & $\begin{array}{c}5-20 \% \\
\text { extraction } \\
48-69 \% \\
\text { carbonation }\end{array}$ & $\begin{array}{l}\text { Vogeli, 2012; } \\
\text { Vogeli et al., } \\
\text { 2011; Meyer, } \\
\text { 2014; Meyer et } \\
\text { al., } 2014 \\
\end{array}$ \\
\hline \multirow{3}{*}{$\begin{array}{c}\text { National Energy } \\
\text { Technology } \\
\text { Laboratory - NETL } \\
\text { (Direct Aqueous) }\end{array}$} & $\begin{array}{l}75 \mu \mathrm{m} \text { heat- } \\
\text { treated } \\
\text { serpentine }\end{array}$ & $\begin{array}{c}0.64 \mathrm{M} \\
\mathrm{NaHCO}_{3} \\
1 \mathrm{M} \mathrm{NaCl} \\
\end{array}$ & $155^{\circ} \mathrm{C}$ & $115 \mathrm{~atm}$ & $>50 \%$ conversion & \multirow{3}{*}{$\begin{array}{l}\text { O’Connor et } \\
\text { al., 2002; } \\
\text { Gerdemann et } \\
\text { al., } 2007\end{array}$} \\
\hline & $\begin{array}{l}75 \mu \mathrm{m} \\
\text { Olivine }\end{array}$ & $\begin{array}{c}0.64 \mathrm{M} \\
\mathrm{NaHCO}_{3} \\
1 \mathrm{M} \mathrm{NaCl} \\
\end{array}$ & $185^{\circ} \mathrm{C}$ & $150 \mathrm{~atm}$ & $80 \%$ conversion & \\
\hline & $\begin{array}{c}75 \mu \mathrm{m} \\
\text { Wollastonite }\end{array}$ & $\begin{array}{c}\text { Distilled } \\
\text { Water }\end{array}$ & $100^{\circ} \mathrm{C}$ & 40 atm ` & $>70 \%$ conversion & \\
\hline
\end{tabular}




\section{Chapter 3}

\section{Methodology}

Based on the literature review and analysis, a number of mineral carbonation processes have been identified for further assessment, in terms of their carbon balance. The selection of the processes was generally made on the basis of attempting to cover several process routes, identifying promising processes within those routes keeping in mind the relatively inert nature of pyroxene considered as feedstock, reaction times as well as recycle and regeneration opportunities. This meets the first objective of the dissertation: "Identify potentially feasible flowsheets for the mineral carbonation of PGM tailings on the basis of literature data and information." The selected processes include:

i. The Ammonium Salts Process - Indirect Aqueous pH Swing

ii. Lackner's $\mathrm{HCl}$ Multi-stage Process - Indirect Aqueous

iii. The ÅAU Process - Indirect Multi-stage Gas/Solid

iv. Mineral Acid pH Swing Process - Indirect Aqueous pH Swing

v. The NETL Process - Direct Aqueous

This chapter outlines the methodology applied to address the second and third objectives of the dissertation:

II. Develop mass and energy balances for the identified processes using process simulation software.

III. Establish the carbon footprint of the selected mineral carbonation processes using a life-cycle based approach.

The development of potentially feasible mineral carbonation process flowsheets involves the use of block flow diagrams, developed into flowsheets, that cover the mass and energy balances of the mineral carbonation process (foreground), as well as the mass and energy balances of process inputs (background). The flowsheets developed are based on data and information reported in available literature (including journal papers, patents, public reports, dissertations and conference proceedings), supported by engineering heuristics and in-house knowledge.

These diagrams form the basis for the development of Aspen Plus ${ }^{\mathrm{TM}}$ V8.0 simulations that are used to compute the mass and energy balance of the mineral carbonation processes. The mass 
and energy balances obtained from Aspen provide process material and energy requirements that are used for background process modelling to evaluate the overall emissions burden of the selected mineral carbonation process. This is conducted through accounting for the $\mathrm{CO}_{2}$ footprint of the heat and electricity requirements of the process, production of process reagents including water, and the compression of $\mathrm{CO}_{2}$ for transportation and process use. The life-cycle impact assessment (LCIA) software SimaPro v7.3.3 is used to conduct this accounting through converting material and energy requirements to $\mathrm{CO}_{2}$ emission equivalents that result from these processes and operations, giving rise to the total $\mathrm{CO}_{2}$ burden for the mineral carbonation process.

This value is then compared to the $\mathrm{CO}_{2}$ that the process sequesters to give an indication of the net carbon footprint of the process. This approach is summarized in the schematic shown on Figure 3-1. Subsequent sub-sections of this chapter provide further information on the goal and scope of the study, and the modelling of mass and energy balances, as well as $\mathrm{CO}_{2}$ emissions accounting. Detailed descriptions of the goal and scope of the study, the mass and energy balance modelling and carbon dioxide emissions accounting are provided in Sections 3.1, 3.2 and 3.3 , respectively.

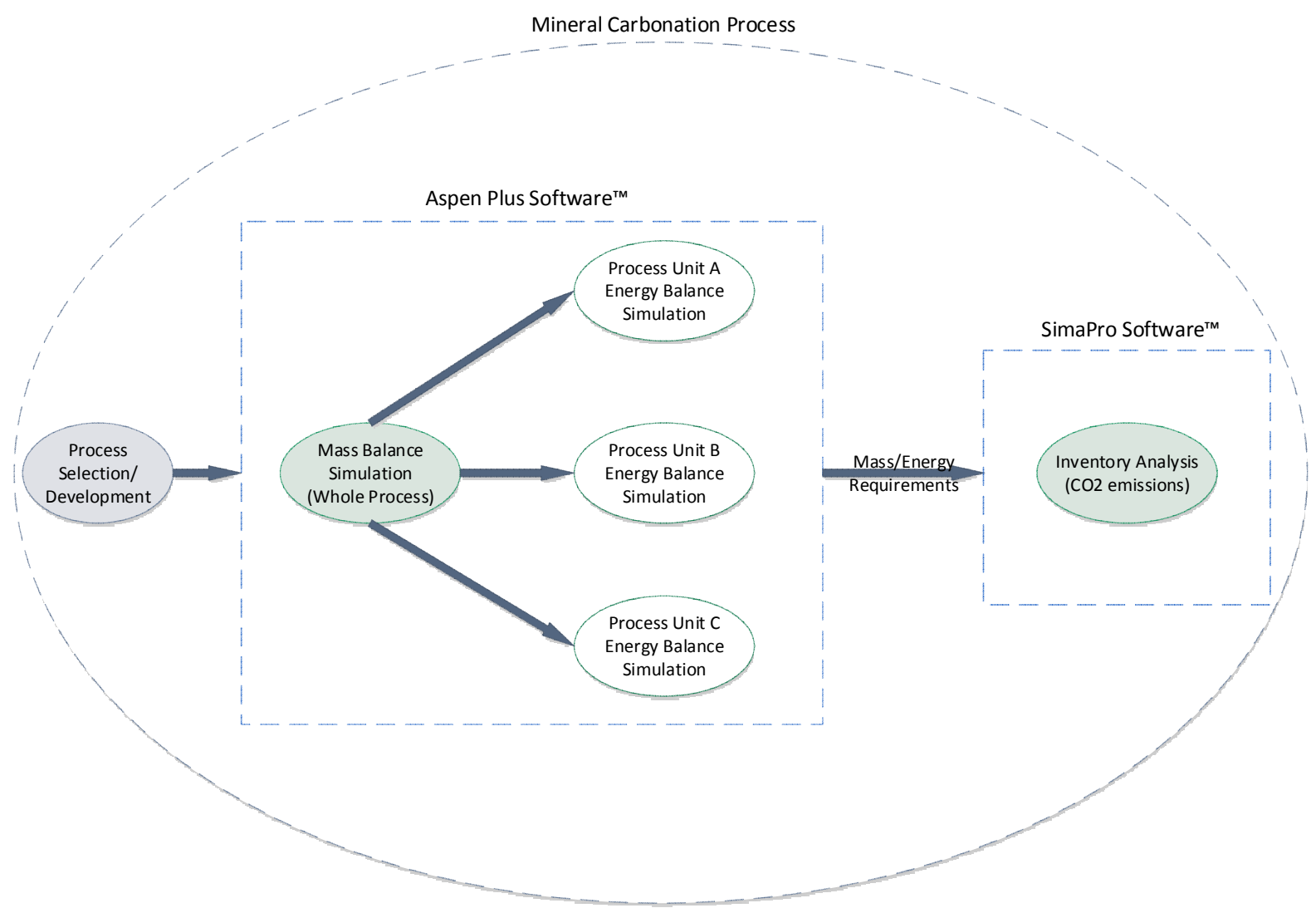




\subsection{Goal, Scope and Limitations}

According to ISO (2006) the goal of a study should include the reasons to carry out the study, the intended application as well as the intended audience. This is also the place where system boundaries for the study are defined and the functional unit stated (Finnveden et al., 2009). The goal of this study is to evaluate selected processes for mineral carbonation in terms of their effectiveness to sequester carbon dioxide, using PGM tailings as the silicate mineral feedstock. This enables the identification of sustainable processes that can be implemented, as well as identify areas that are burdensome to the process. This will aid researchers in focusing research efforts on promising routes, eliminate unsustainable processes, and provide viable options for decision makers interested in implementing these processes in South Africa.

Streamlining, boundaries and assumptions are crucial (Khoo, 2007) since it is impossible to include every single facet of the processes' life cycle stages. The system boundary used in this study could be classed as that "between the technical system and the environment", using classifications defined by Guinée et al. (2002). Figure 3-2 shows the system boundary defined for the evaluation of the mineral carbonation process routes. This indicates the key inputs to the process, and demonstrates that this boundary is consistent with a cradle-to-gate approach.

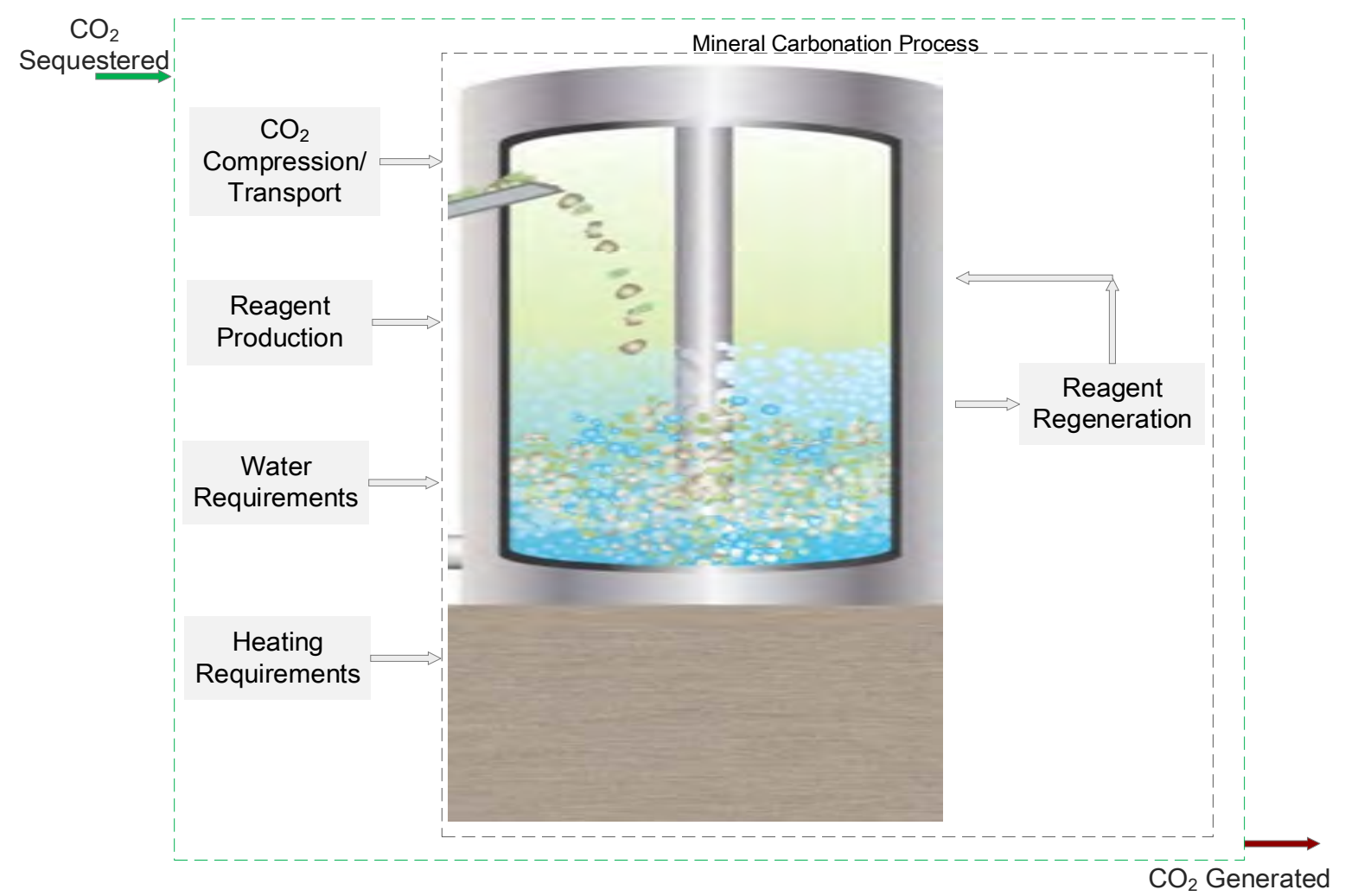


This boundary covers impacts from background processes such as generation of heat through the combustion of methane, electricity for the compression of carbon dioxide for transport and plant use, water and chemical reagents required. Regeneration of reagents and its impact are also taken into consideration. The system boundary ends at the point in which the mineral carbonation products are formed, and does not take into account downstream processing or utilization of these products. The transportation of PGM tailings is not considered since it is assumed that the mineral carbonation plant will be located close to the tailings dumps. The effect of mining and processing of the silicate mineral (PGM tailings) used is not considered since this stream is obtained as a waste stream from platinum production and is common to all the mineral carbonation process. It is assumed that this material is considered to be suitable for processing as is, and that no further grinding and/or dewatering will be required. The disposal and treatment of waste is not considered because the carbonate compounds are stable, and the land used to collect the tailings could be used to store the carbonation products. Furthermore, the impact of materials of construction of the mineral carbonation plant have not been considered.

The functional unit is a quantitative description of the performance of the product system or systems (Rebitzer et al., 2004). It describes the primary function fulfilled by the product system (Guinée et al., 2002). This allows for systems or processes to be treated as functionally equivalent and thus comparable, which enables selection of promising processes. Considering that the primary function of the mineral carbonation process is the sequestration of carbon dioxide, the functional unit of $1000 \mathrm{~kg}$ of carbon dioxide sequestered was selected. As a consequence of this the impact category selected was the climate change category.

The primary limitation to the data used in this study is that, as has been discussed in Section 3.1 , the majority of the proposed processes have been based on wollastonite, olivine and serpentine. The primary component of the PGM tailings feedstock is pyroxene (Vogeli et al., 2011; Meyer et al., 2014). This presents challenges with regards to the chemical reactions and conversions that could be attained with PGM tailings as a feedstock. The first challenge has been overcome by making the assumption that pyroxene will react to produce similar products to olivine, since they are chemically similar. To overcome the second challenge, it was noted that pyroxene is less reactive in comparison to the other silicate minerals (Meyer, 2014). This required conservative estimates of conversion to be assumed, in line with this observation. 


\subsection{Mass and Energy Balance Modelling}

The evaluation of material and energy balances is conducted using Aspen Plus software v8.0. Aspen Plus ${ }^{\mathrm{TM}}$ is a robust chemical process simulation software that allows for process model development and simulation. This includes the ability to specify components, thermodynamic models, process units as well as operating conditions. As a result, this enables the prediction of process behaviour, the opportunity to run different scenarios and conduct "what if" analyses, performing sensitivity analyses, as well as allowing for process optimisation (Aspen Plus Manual, v8). A general approach is taken to the development of mass and energy balance simulations, though each particular process has specific details involved with them. A summary of general assumptions is initially discussed.

\subsubsection{General Assumptions and Approach for Model Development}

The recommended approach in developing simulations on Aspen is to do so in a block-byblock manner, that is, building the simulation gradually and running it every time a new unit is built into the model. Sinnot and Towler (2008) refer to this approach as to "creep up on" the solution. This makes it easier to troubleshoot and find sources of errors in the model, because if errors arise after a particular change to a working simulation, then the source of errors is associated with that change. This is particularly crucial in complex simulations that involve numerous process units and recycle streams, which may be vulnerable to convergence problems.

To take it a step further, the simulations are further broken down into separate mass and energy balance simulations. The mass balance for the whole process is conducted separately, and then energy balances based on that mass balance are conducted for individual process units or smaller groups of units. The benefit of this approach is that it makes identifying bugs in the simulations easier whilst also reducing computation time for the simulations.

The basis for the material balance was $1 \mathrm{ton} / \mathrm{hr}$ of carbon dioxide fed into the process. This process flow rate was selected a result of the functional unit, and for the ability to compare results to other processes and studies on the basis of emissions released per ton sequestered. Assuming perfect scale-up, the flows can then be adjusted to satisfy a future supply-specific (e.g. annual output of $\mathrm{CO}_{2}$ from Sasol, Secunda) process design should the process demonstrate sustainability on the basis of a unit $\mathrm{CO}_{2}$ sequestered. The mineral feedstock to the proposed mineral carbonation process will be PGM tailings sourced from mining operations of companies like BRPM, Impala, Northam and Lonmin. This feedstock is available as fine- 
milled particles $(<150 \mu \mathrm{m})$ according to Vogeli et al. (2011), thus it is assumed it will require no further grinding or milling, as discussed earlier. The major component ( $\sim 65 \mathrm{wt} \%)$ in PGM tailings is pyroxene (Vogeli, 2011; Meyer, 2014). For the sake of simplicity, the feed material is thus assumed to be pyroxene (enstatite) in all process systems studied here.

The impact of the transport of mineral feedstock was assumed to be negligible since the mineral carbonation plant would be located close to this feedstock. The pumping of materials, separations and mixing impacts were not included in energy calculations as these were assumed to be much smaller than heating and compression. Separations of phases and components were assumed to perfect for simplicity, with the recognition that this wouldn't be the case in practice. The selection of the type of separation process depends on the differences in the physical or chemical properties of the components. The effectiveness of the separation between phases/components depends on the exploitation of differences in molecular, thermodynamic and transport properties (Seader and Henley, 2006). Since this is a preliminary study of these processes, the level of detail required in this analysis was considered to be outside the scope of this study. The compression took into account the compression for transport by pipeline as well as any additional compression required to raise the pressure from the pipeline pressure for process purposes. In the case where processes operations occurred at lower pressures than the pipeline pressure, a turbine was used to reduce pressure thus generating an electricity credit.

Due to the absence of processes based on pyroxene, the processes are assumed to exhibit similar chemistry to these minerals, with regards to how they react (as suggested in literature/patent), though the conversions (single pass) are adjusted according to those obtained in literature for pyroxene, as has been discussed in Section 3.1. In the absence of experimental data, conservative estimates of these single pass conversions are made, particularly for the leaching step. Conversions of other processes excluding carbonation and dissolution are assumed to $100 \%$. Process specific assumptions are outlined in the relevant sub-sections of the Chapter 4.

\subsubsection{Property Methods Selection}

A property method is a collection of routes for the calculation of properties needed by unit operation models. The selection of the appropriate method for estimating thermodynamic properties of pure components and mixtures is one of the most important steps in the development of a simulation. This decision affects the entire simulation, and the results that will be produced when the simulation is conducted. To ensure meaningful results are obtained 
from simulations, it is vital to ascertain that the property method selected is representative of the system being modelled.

\section{Aqueous Operations}

The Aspen Physical Property System manual recommends electrolyte activity coefficients models for mineral leaching and hydrometallurgical operations (AspenTech, 2013). The most versatile of these models is the ELECNRTL property method. Due to the presence of electrolytes in these processes, the thermodynamic model selected to model aqueous systems in the selected carbonation processes was the ELECNRTL property method. This property method can handle electrolyte systems with very low and high concentrations. This is achieved through binary parameters that model the interactions of true species in aqueous single electrolyte systems, and multicomponent systems. Another strength of this model is that it is effective across a wide range of temperatures (AspenTech, 2013). In this model, the liquid phase and heats of mixing are modelled through the electrolyte NRTL model, whereas the vapour phase properties are modelled through the Redlich-Kwong equation of state. A number of unit operations across the mineral carbonation process routes are of aqueous nature. This means that a majority of the process units will be modelled using this property method, with the exception of specific operations like the compression of $\mathrm{CO}_{2}$ and the gas-solid reactions in the $\AA A U$ process. The property method selected for each unit operation will be presented in the table for input specifications in Appendix A:.

\section{Gaseous and Gas-Solid Operations}

Though most processes in the proposed mineral carbonation routes are of an aqueous nature, some unit processes within these routes cannot be classified as such. This means that the property method used for these processes needs to be appropriate to the unit it attempts to describe. Carbon dioxide that needs to be sequestered by the process has to be compressed in order to be transported by pipeline to the carbonation site, and for use in unit operations of some of the mineral carbonation processes. To model the compression of the gas, the RKSOAVE property method was selected. The Redlich-Kwong-Soave (RKS) equation of state model is appropriate for gas processing of hydrocarbons and light gases, such as carbon dioxide, hydrogen sulphide and hydrogen (AspenTech, 2013). The carbonation reaction in some process routes ( $\mathrm{HCl}$ extraction, AAU process) involves a gas-solid reaction between carbon dioxide and magnesium hydroxide. The extraction process in the ÅA process can also be classified as a gas-solid process, since ammonium sulphate decomposes into gas phase at the reaction temperature. The UNIQUAC property method was selected to model these process 
units. This model is an activity coefficient model that is capable of handling any combination of polar and non-polar compounds, up to very strong non-ideality (AspenTech, 2013).

\subsubsection{Simulation Development Illustrations}

The development of simulations for processes is based on process routes proposed in literature. The flowsheets of these processes are developed and built up on the Aspen Plus simulation package. The resulting simulation diagrams are presented in Appendix E for each mineral carbonation process considered.

The chemical components that are involved in the particular process are selected and input into the "Components" tab as indicated in Figure 3-3. The components added into this tab are not just reagents but also include products of process units as well as the mineral carbonation process in general.

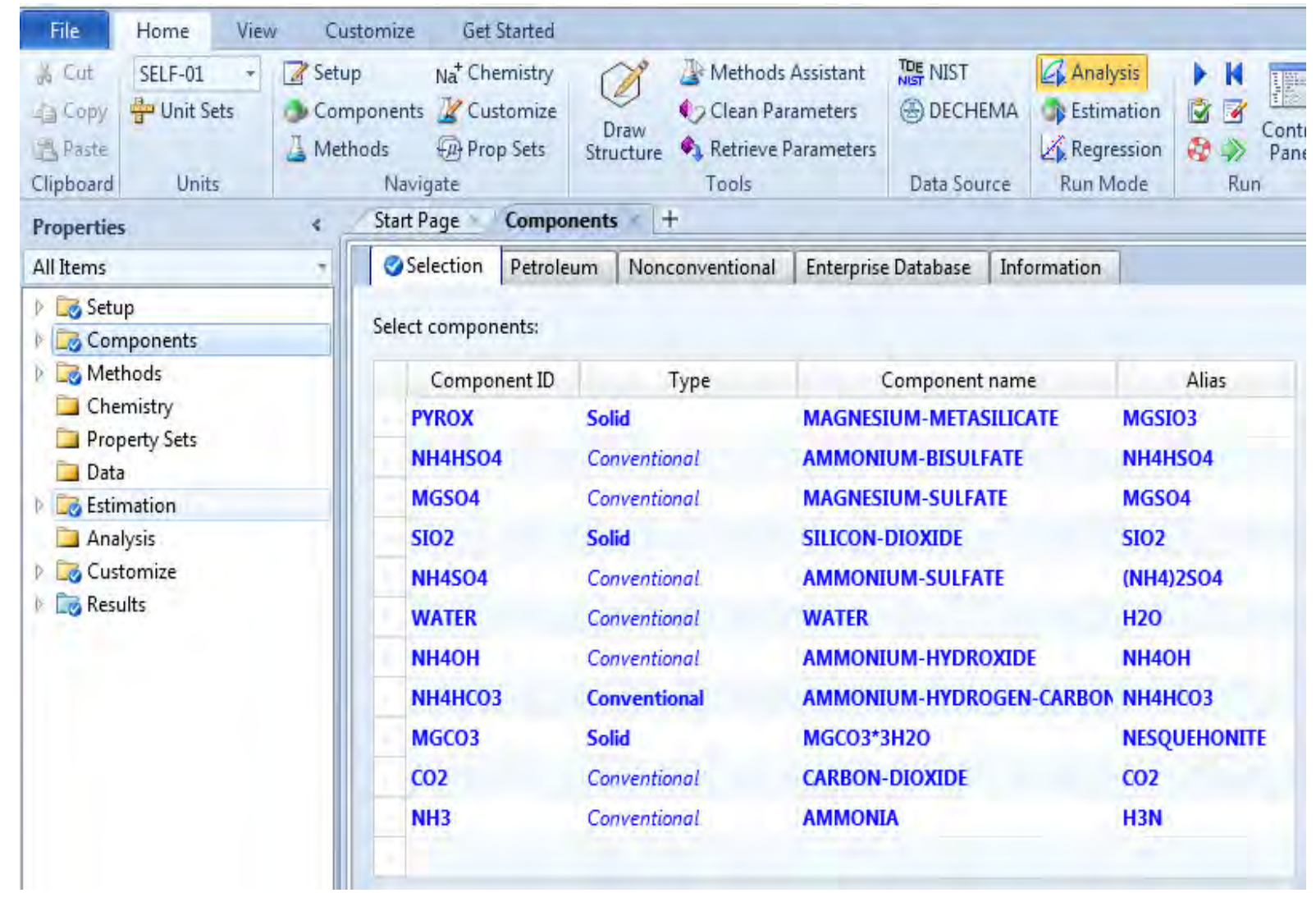

Figure 3-3: Chemical component input on Aspen Plus simulation software

The next step is the definition of the property method used in the process shown in Figure 3-4. As discussed in Section 3.2.2, the property method used for aqueous processes was the ELECNRTL property method, and for gas and gas-solid operations the RK-SOAVE and UNIQUAC property methods can be used. These can be selected by navigating to the "Methods" tab and browsing through the list of property methods on Aspen until the 
appropriate method is found. These two steps fall in the "Properties" tab of the simulation interface, and clicking next will run the properties package, as configured by the user, in preparation for the simulation. The next step is to navigate to the simulation environment by moving to the "Simulation" tab. Here, the simulation diagrams presented in Appendix E are developed. Each process unit block and process stream has to be initialized with input data in order to complete the simulation. The input data for initializing all the simulations is presented in Appendix A.

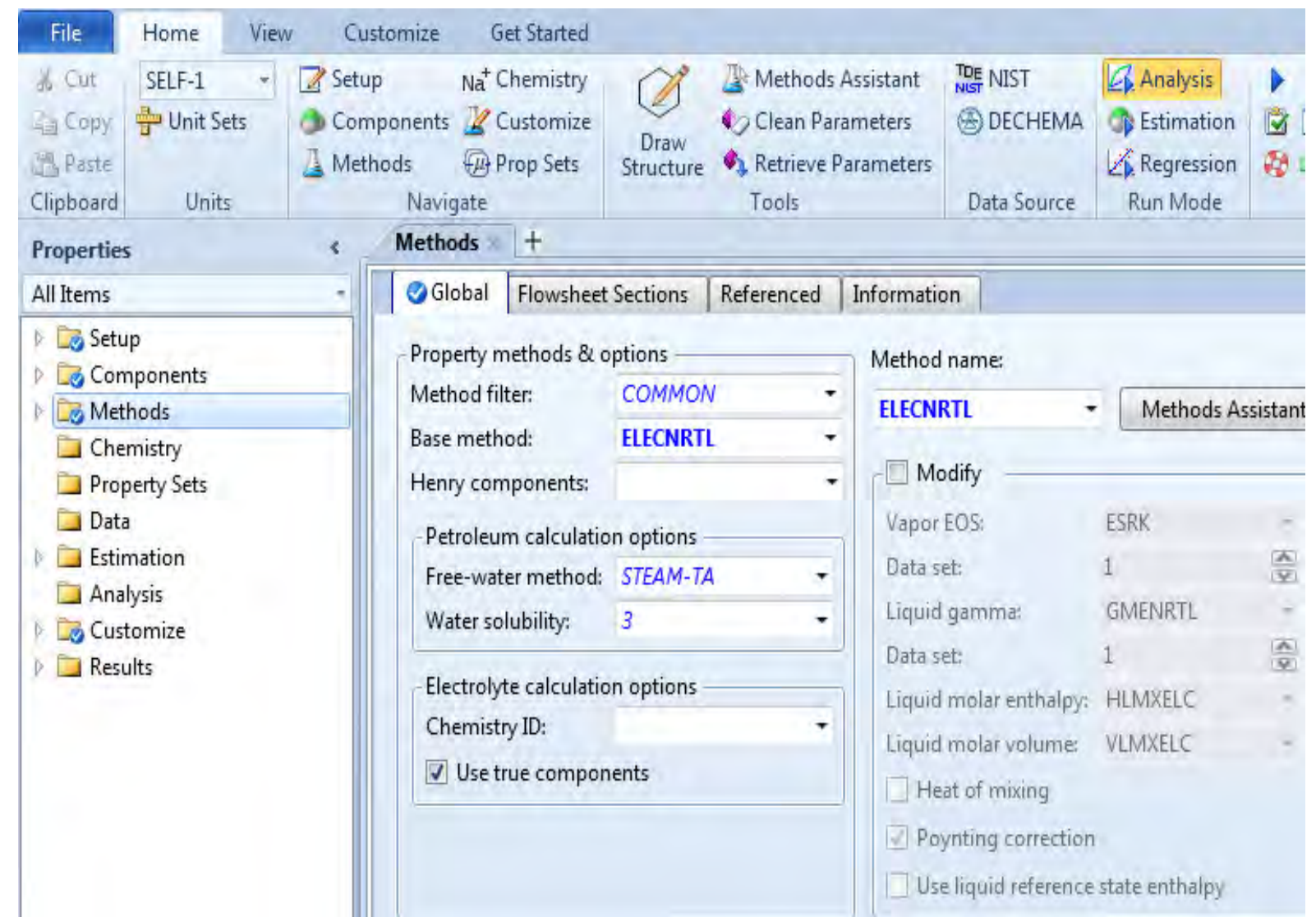

Figure 3-4: Property method selection Tab on Aspen Plus simulation package

\subsection{Carbon Dioxide Emissions Accounting}

The material and energy requirements of the mineral carbonation process serve as the basis for the calculation of carbon dioxide footprint that will result from the implementation of the selected process. These include emissions associated with the production of chemical reagents, supply of water, generation of process heat, and supply of electricity for the compression of carbon dioxide. To determine these contributions the sources and processes used to generate these requirements need to be determined, in order to use background process modelling software to establish the emissions associated with the requirements. The first sub-section (3.3.1) of this section will deal with establishing sources and processes for generating process 
requirements, whereas the second subsection (3.3.2) will discuss the approach taken to calculate resulting emissions.

\subsubsection{Inventory Analysis}

The discussion of input materials into the mineral carbonation process has been separated into two parts; the discussion of those inputs that are common in all processes, and the discussion of those that are specific to certain processes.

\section{Common Process Inputs}

\section{Carbon Dioxide}

The $\mathrm{CO}_{2}$ stream feeding into the mineral carbonation process is assumed to be a pure stream of $\mathrm{CO}_{2}$, since the SASOL synfuels process produces a high purity $\mathrm{CO}_{2}$ stream (Cloete, 2006; Vogeli et al., 2011). All carbonation processes are developed on the basis of $1 \mathrm{ton} / \mathrm{hr}$ of $\mathrm{CO}_{2}$ sequestered. Since the synfuels plant and the mine tailings site are not located on the same site, in fact about $300 \mathrm{~km}$ apart, transport of materials is necessary. According to Giannoulakis et al. (2014) it is more preferable to transport $\mathrm{CO}_{2}$ to the mine site than transporting the large quantities of rock to the emissions site. This implies that options for the transport of $\mathrm{CO}_{2}$ need to be considered.

The transport of $\mathrm{CO}_{2}$ can be carried out through tanks, ships or pipelines. The selection of the method of transport is dependent on the volume being transported and the distance (IPCC, 2005; Leung et al., 2014). The most preferable method for transporting carbon dioxide through long distances on land is by the use of pipelines (Svensson et al., 2004; Koornneef et al., 2010). This is because motor carriers and railways are expensive and lack capacity (Svensson et al., 2004). Transportation of $\mathrm{CO}_{2}$ by pipelines is not new (IPCC, 2005) with a network of $2500 \mathrm{~km}$ in the United States having been implemented for the purposes of enhanced oil recovery (EOR). In South Africa, Sasol currently operates a natural gas pipeline from the Temane and Pande gas fields in Mozambique (PWC, 2012). The knowledge from operating natural gas and hydrocarbon pipelines can be used for implementation of $\mathrm{CO}_{2}$ pipelines (Svennson et al., 2004), since transportation conditions are similar.

Transportation of $\mathrm{CO}_{2}$ at low pressures is possible, but due to low density of the fluid in these conditions, the capacity required to transport meaningful amounts of $\mathrm{CO}_{2}$ would be substantial. The more preferable option is to transport it in supercritical state, where it has a high density as liquids but viscosity similar to that of gases (Wildbolz, 2007; Leung et al., 2014). The recommended conditions for the transportation of carbon dioxide are between $13{ }^{\circ} \mathrm{C}$ and $44{ }^{\circ} \mathrm{C}$ 
and pressures above the critical point ( 73.9 bar and $\left.31.1^{\circ} \mathrm{C}\right)$, typically between $85 \mathrm{~atm}$ and 149.6 atm (WRI, 2008, Leung et al., 2014). This pressure can then be throttled up or down depending on process requirements using either a compressor or turbine respectively, as discussed earlier. Literature suggests recompression is necessary every $150 \mathrm{~km}$, but practice has shown transport without recompression for distances above $400 \mathrm{~km}$ (Wildbolz, 2007). Since the transport distance is $300 \mathrm{~km}$ (less than the $400 \mathrm{~km}$ ), it is assumed that recompression will not be necessary.

\section{$\underline{\text { Heat, Electricity and Water }}$}

A number of unit operations in the mineral carbonation process require heat to be supplied to them. Industrial operations typically use fossil fuels (coal, natural gas, oil) and biomass (bagasse, vegetable oil, wood chips) to generate heat (Thekdi, 2007). Gaseous fuels such as natural gas are commonly preferred for use in industrial heating since they burn cleanly and completely without producing soot particles (Baukal, 2000; Liang et al., 2012). Natural gas is also less costly per unit of energy (Liang et al., 2012) in comparison to oil and coal. The natural gas pipeline from Mozambique carries 240 million gigajoules per annum of which about half is used by Sasol in the GTL process, with the balance being available for commercial and industrial customers (PWC, 2012). For the purposes of providing heat, for all selected mineral carbonation process routes, natural gas was selected as the supply due to the reasons outlined. Some other unit operations in the mineral carbonation process, such as compression, require electricity. The proposed mineral carbonation plant will be located in South Africa, and the electricity mix selected consistent with the South African scenario. For all cases where water was required, process water from surface water was assumed as the source.

\section{Process Specific Inputs}

\section{Ammonium Bisulphate}

The ammonium salts based $\mathrm{pH}$ swing process proposed by Wang and Maroto-Valer (2011) makes use of ammonium bisulphate for the extraction of cations from the silicate mineral. This compound is formed when ammonium sulphate salt is heated in an open system to temperatures above $100{ }^{\circ} \mathrm{C}$, which causes it to decompose into ammonium bisulphate and ammonia (KirkOthmer, 2007). The authors of the ammonium salts mineral carbonation process patent also suggest the use of this mechanism to regenerate ammonium bisulphate (Wang and MarotoValer, 2013). The production of ammonium bisulphate for use in the mineral carbonation process will be assumed to be from the use of ammonium sulphate as a feedstock. 


\section{Ammonium Sulphate}

This chemical compound is used to extract the magnesium from the silicate mineral through the process proposed at Åbo Akademi Univesity (Fagerlund et al., 2012; Nduagu, 2012). According to Kirk-Othmer (2007), ammonium sulphate is produced through direct neutralisation of aqueous sulphuric acid with gaseous ammonia. This process will be assumed to be the means of production of ammonium sulphate for use in the Åbo Akademi process.

\section{Ammonium Hydroxide}

Ammonium hydroxide, which is a solution of ammonia in water, is used to adjust the $\mathrm{pH}$ in the ammonium salts process (Wang and Maroto-Valer, 2011). The production of ammonia occurs through the reaction of nitrogen gas with hydrogen gas, in a fairly simple reaction originally developed by Fritz Haber and Carl Bosch (Appl, 2005). This method provides for $90 \%$ of the world's production of ammonia according to Appl (2005). The challenge is typically the production of hydrogen to be used for this process (Kirk-Othmer, 2007) with the majority of the ammonia plant dedicated to hydrogen production. In most global operations the production of hydrogen is done through steam reforming using natural gas as a feedstock. The ammonium hydroxide to be used for mineral carbonation according to the ammonium salts process will be assumed to have been provided through ammonia produced via the steam reforming route as described above.

\section{Hydrochloric Acid}

Also known as muriatic acid, this is a solution of hydrogen chloride in water. Hydrochloric acid is used to extract magnesium from the silicate mineral in the multi-stage extraction process (Lackner et al., 1995) and the indirect pH swing method used by Meyer et al. (2014). The production of hydrogen chloride occurs through synthesis from hydrogen and chlorine gases. This process occurs in an industrial burner and is considered the simplest approach (Austin and Glowacki, 2005). According to Kirk-Othmer (2007) a high purity ( $>99 \%)$ hydrogen chloride gas stream is produced from the burner, which can be used to manufacture pure hydrochloric acid. The direct synthesis from elements was selected as the method of production for the hydrochloric acid used in the mineral carbonation processes discussed here.

\section{$\underline{\text { Sodium Hydroxide }}$}

This chemical compound is used to adjust the $\mathrm{pH}$ to alkaline conditions in the indirect $\mathrm{HCl}$ leach process (Meyer et al., 2014). The production of sodium hydroxide primarily occurs through the electrolysis of sodium chloride (Kirk-Othmer, 2007). Three different cell 
configurations are used for this process: the mercury cell, the diaphragm cell and the membrane cell. The application of either of these technologies produces sodium concentration with the same final level of purity (Kurt and Bittner, 2005). The approach taken for the sodium hydroxide used in the mineral carbonation is to use an average of the three processes as this is allowable by the software tool used for carbon dioxide emissions accounting.

\section{$\underline{\text { Sodium Chloride }}$}

Commonly known as salt, this compound is produced from extracting the salt by using water to dissolve it from rock salt deposits underground. The resulting brine solution is then evaporated to produce sodium chloride crystals (Kirk-Othmer, 2007). This process is known as solution mining. It is considered the modern, economical method for extracting sodium chloride from underground deposits (Westphal et al., 2005). Sodium chloride is used as an additive to the National Energy Technology Laboratory (NETL) direct aqueous carbonation process to enhance process performance (O'Connor et al., 2002; Gerdemann et al., 2007). The source of sodium chloride for this mineral carbonation process route will thus be through the solution mining process discussed.

\section{$\underline{\text { Sodium Bicarbonate }}$}

The second additive to the direct aqueous carbonation process developed at the NETL is sodium bicarbonate. This compound is produced primarily through the use of a process implemented in 1880 called the Solvay process (Thieme, 2005). This process involves the reaction of sodium chloride with ammonia and carbon dioxide to produce sodium bicarbonate and ammonium chloride in the presence of water. Typically, this process includes a calcination step that produces sodium carbonate from sodium bicarbonate (Kirk-Othmer, 2007). The production of sodium bicarbonate for use in the direct aqueous mineral carbonation process is assumed to be through the Solvay process as has been discussed.

\subsubsection{Calculation of Emissions}

Impact assessment is the phase where the results of the inventory analysis are processed and interpreted in terms of environmental impacts. The impact assessment attempts to establish a link between a process and its potential environmental impact (EPA, 2006). The identification of relevant impact categories is the foremost step in the impact assessment, and is typically conducted as part of the goal and scope definition. Accordingly, the climate change impact category was considered in the analysis as has been stated in Section 3.1. This impact is defined as the impact of human emissions on radioactive forcing of the atmosphere (Guinée et al., 
2002). These emissions are measured through the emissions of greenhouse gases $\left(\mathrm{CO}_{2}, \mathrm{CH}_{4}\right.$, CFCs, $\mathrm{CO}, \mathrm{HFCs}, \mathrm{O}_{3}$ ) into the atmosphere. The accumulation of these gases in the atmosphere causes climate change. Science-based characterisation factors are used to convert and combine results into representative indicators of impacts. In the case of climate change greenhouse gas emissions associated with material and energy inputs are expressed in terms of $\mathrm{CO}_{2}$ equivalents. This is a measure used to define greenhouse gas emissions in terms of a common unit, which is related to the heat the greenhouse gas traps relative to carbon dioxide (IPCC, 2007). This is referred to as the Global Warming Potential (GWP), and Table 3-1 shows the potentials for a 100-yr time horizon as provided by the IPCC (2007).

Table 3-1: Global Warming Potentials (GWP100) for greenhouse gases (IPCC, 2007)

\begin{tabular}{|l|c|}
\hline \multicolumn{1}{|c|}{ Greenhouse Gas } & $\begin{array}{c}\text { Global Warming Potential } \\
(\mathbf{1 0 0}-\mathbf{y r} \text { horizon) }\end{array}$ \\
\hline Carbon Dioxide $\left(\mathrm{CO}_{2}\right)$ & 1 \\
\hline Methane $\left(\mathrm{CH}_{4}\right)$ & 25 \\
\hline Nitrous oxide $\left(\mathrm{N}_{2} \mathrm{O}\right)$ & 298 \\
\hline Hydrofluorocarbons $(\mathrm{HFCs})$ & $124-14800$ \\
\hline Chlorofluorocarbons $(\mathrm{CFCs})$ & $4750-14400$ \\
\hline Sulphur hecafluoride $\left(\mathrm{SF}_{6}\right)$ & 22800 \\
\hline Nitrogen trifluoride $\left(\mathrm{NF}_{3}\right)$ & 17200 \\
\hline
\end{tabular}

The mass and energy balance results obtained on Aspen serve as a basis for analysis in terms of the $\mathrm{CO}_{2}$ footprint that results from the material and energy requirements of the process. This analysis is conducted using SimaPro software v7.7.3. SimaPro is a product system modelling and assessment software that is widely used as a life cycle assessment tool to give an indication of the sustainability of a product or process (Herrmann and Moltesen, 2015). The software program comes integrated with a number of databases including Ecoinvent v2.2, which is the world's leading database. This database provides consistent and transparent up-to-date Life Cycle Inventory (LCI) data, and was used for assessing the impacts of heat and chemical reagents. For electricity, the South African electricity mix database developed by Dick (2012) at SASOL was used, and the ELCD database, which is integrated on SimaPro, for process water.

The approach taken for defining category indicators is the problem-oriented approach. According to Guinée et al. (2002) this approach is driven by environmental problems (midpoint) rather than by damage (endpoint). The uncertainty of results using this approach is low in comparison to the endpoint level, since category indicators are defined at midpoints 
along the environmental mechanism. Hence, this approach is considered the "best available practice" (Guinée et al., 2002). A representation of the midpoint and endpoint approaches on climate change is presented in Figure 3-5.

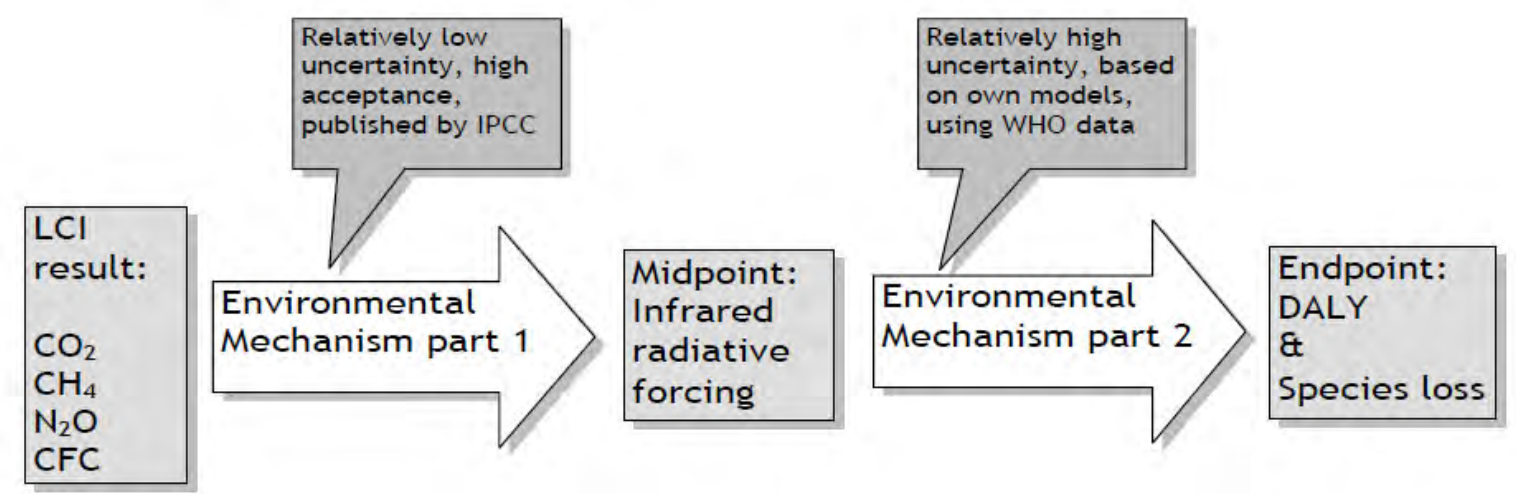

Figure 3-5: A representation of midpoint and endpoint approach to climate change (Goedkoop et al., 2013)

The ReCiPe Midpoint $(\mathrm{H})$ method, which is the most frequently used, was used for impact assessment. This method uses the global warming potential as the characterisation factor for the climate change category (PRé, 2015). The carbon dioxide equivalency factors are calculated using the following equation (Goedkoop et al., 2013):

$$
\operatorname{GWP}_{\mathrm{x}, \mathrm{T}}=\frac{\int_{0}^{\mathrm{T}} \mathrm{a}_{\mathrm{x}} \times[\mathrm{x}(\mathrm{t})] \mathrm{dt}}{\int_{0}^{\mathrm{T}} \mathrm{a}_{\mathrm{r}} \times[\mathrm{r}(\mathrm{t})] \mathrm{dt}}
$$

Where $G W P_{x, T}$ is the global warming potential of substance $x$ over time horizon $T, a_{x}$ is the radioactive efficiency due to a unit increase in atmospheric abundance of the substance in question, $[x(t)]$ is the time-dependent abundance of substance $x$ and the corresponding quantities in the denominator being the reference gas. The factor for this method is for a 100yr time horizon (GWP100) for each greenhouse gas emission to the atmosphere. The equivalence factors presented in the IPCC (2007) report are used as midpoint characterisation factors (Goedkoop et al., 2013) to estimate global warming potential.

The materials and energy requirements are used as input data in developed SimaPro process blocks. These blocks are developed on the basis of the considerations of the source of the particular requirement, as discussed in Section 3.3.1. The detailed outlines of SimaPro process inputs and resulting emissions are presented in Appendix D. 


\section{Chapter 4 \\ Case Study Results}

The primary aim of this study is to investigate the viability of using PGM tailings to sequester $\mathrm{CO}_{2}$ from the perspective of an overall $\mathrm{CO}_{2}$ balance across the entire process. The results obtained from the evaluation of selected mineral carbonation process routes are presented in this Chapter. Mass and energy balance calculations were conducted on selected carbonation processes, through which the carbon dioxide footprints of these processes were then evaluated. As discussed in Chapter 3, pyroxene was used as a proxy for PGM tailings and, in the absence of available experimental data, conversions derived from stoichiometric equations and assumed efficiencies. On this basis, material requirements for processes are presented, on which energy calculations were based. The energy requirements of individual units are then presented, which identify the major energy consuming units. To conduct the mass and energy balances, AspenPlus simulation software was used as detailed in Section 3.2. It is important to note that the reactor conversions referred to in this Chapter are single pass conversion of materials as they pass the reactor. The overall conversions for the process have been attached in Appendix F. Finally, results on the $\mathrm{CO}_{2}$ footprint of the process are presented. These results were generated using material and energy balance data processed through SimaPro software, to give carbon dioxide footprints as described in Section 3.3.

\subsection{Ammonium Salts Process}

\subsubsection{Flowsheet Description and Mass Balance}

The ammonium salts process involves the use of ammonium salts based reagents to extract and carbonate metal cations and also to capture $\mathrm{CO}_{2}$. The key chemical reactions occurring are presented in Table 4-1, and a simplified version of the flowsheet and material balance for the ammonium salts process is presented in Figure 4-1. This diagram indicates the key flows within the process, as well as the key unit operations that make up the process, for the sake of clarity and simplicity. It does not represent all the flows through the process. This is the case for all processes discussed in this Chapter. Detailed material balances and the Aspen Plus simulation diagram for this process are attached in Appendix B and E, respectively. 
Table 4-1: Chemical reactions occurring in the ammonium salts process

\begin{tabular}{|l|c|c|c|}
\hline Reactor Unit & Chemical Reaction & $\begin{array}{c}\text { T } \\
\left({ }^{\circ} \mathbf{C}\right)\end{array}$ & $\begin{array}{c}\text { P } \\
\text { (atm) }\end{array}$ \\
\hline $\begin{array}{l}\text { Mineral } \\
\text { Dissolution }\end{array}$ & $\mathrm{MgSiO}_{3}+2 \mathrm{NH}_{4} \mathrm{HSO}_{4} \rightarrow \mathrm{MgSO}_{4}+\mathrm{SiO}_{2}+\left(\mathrm{NH}_{4}\right)_{2} \mathrm{SO}_{4}+\mathrm{H}_{2} \mathrm{O}$ & 90 & 1 \\
\hline pH Adjustment & $\mathrm{NH}_{4} \mathrm{HSO}_{4}+\mathrm{NH}_{4} \mathrm{OH} \rightarrow\left(\mathrm{NH}_{4}\right)_{2} \mathrm{SO}_{4}+\mathrm{H}_{2} \mathrm{O}$ & 25 & 1 \\
\hline $\begin{array}{l}\text { Mineral } \\
\text { Carbonation }\end{array}$ & $\mathrm{MgSO}_{4}+\mathrm{NH}_{4} \mathrm{HCO}_{3}+\mathrm{H}_{2} \mathrm{O} \rightarrow \mathrm{MgCO}_{3} \cdot 6 \mathrm{H}_{2} \mathrm{O}+\left(\mathrm{NH}_{4}\right)_{2} \mathrm{SO}_{4}+\mathrm{CO}_{2}$ & 80 & 1 \\
\hline $\mathrm{CO}_{2}$ Capture & $\mathrm{CO}_{2}+\mathrm{NH}_{3}+\mathrm{H}_{2} \mathrm{O} \rightarrow \mathrm{NH}_{4} \mathrm{HCO}_{3}$ & 10 & 1 \\
\hline Regeneration & $\left(\mathrm{NH}_{4}\right)_{2} \mathrm{SO}_{4} \rightarrow \mathrm{NH}_{3}+\mathrm{NH}_{4} \mathrm{HSO}_{4}$ & 300 & 1 \\
\hline
\end{tabular}

Some aspects of this discussion will refer to streams that have been presented only on the simulation diagram attached in the appendices. Simulations for this process were run for extraction efficiencies of $30 \%, 50 \%$ and $90 \%$, which represent the literature extraction efficiency, a gradual improvement in this efficiency as well as an optimistic substantial improvement, respectively. The carbonation efficiency was held at $90 \%$, as suggested in literature (Wang and Maroto-Valer, 2013). The mass balance presented in Figure 4-1 is for a process that sequesters $1000 \mathrm{~kg} / \mathrm{hr}$ of carbon dioxide at $30 \%$ extraction efficiency and $90 \%$ carbonation efficiency, per pass.

Ammonium bisulphate and the silicate mineral are first preheated to the reaction temperature, $90{ }^{\circ} \mathrm{C}$, prior to being fed into the dissolution reactor (MIN-DIS). In this reactor, 30\% conversion of the pyroxene fed in to the reactor, through reaction with ammonium bisulphate, produces $2025 \mathrm{~kg} / \mathrm{hr}$ of magnesium sulphate. The resultant slurry of leach solution, unreacted mineral and silica is then separated in a solid-liquid separation unit (SIO2-SEP). This splits the slurry stream into two streams, a solids stream of which $80 \%(6620 \mathrm{~kg} / \mathrm{hr})$ is recycled $(\mathrm{SIO} 2-$ PYR) and a solution containing magnesium ions (LEACH-1), to improve the overall conversion of pyroxene.

The mineral dissolution unit produces $2025 \mathrm{~kg} / \mathrm{hr}$ of magnesium sulphate in a solution containing excess ammonium bisulphate $(1461 \mathrm{~kg} / \mathrm{hr})$ that needs to be neutralised and $\mathrm{pH}$ adjusted using ammonium hydroxide prior to carbonation. Upon $\mathrm{pH}$ adjustment, which occurs at $25{ }^{\circ} \mathrm{C}$, the solution, now referred to as LEACH-3, is preheated to the carbonation reaction conditions $\left(80^{\circ} \mathrm{C}, 1 \mathrm{~atm}\right)$. Another stream, the ammonium bicarbonate stream (NH4-HCO3) is also fed (after preheating) into the carbonation reactor (MIN-CARB). This stream carries 2993 $\mathrm{kg} / \mathrm{hr}$ of ammonium bicarbonate produced during $\mathrm{CO}_{2}$ capture. It is the carbon dioxide carrier, providing the essential ingredient required for carbonation. 


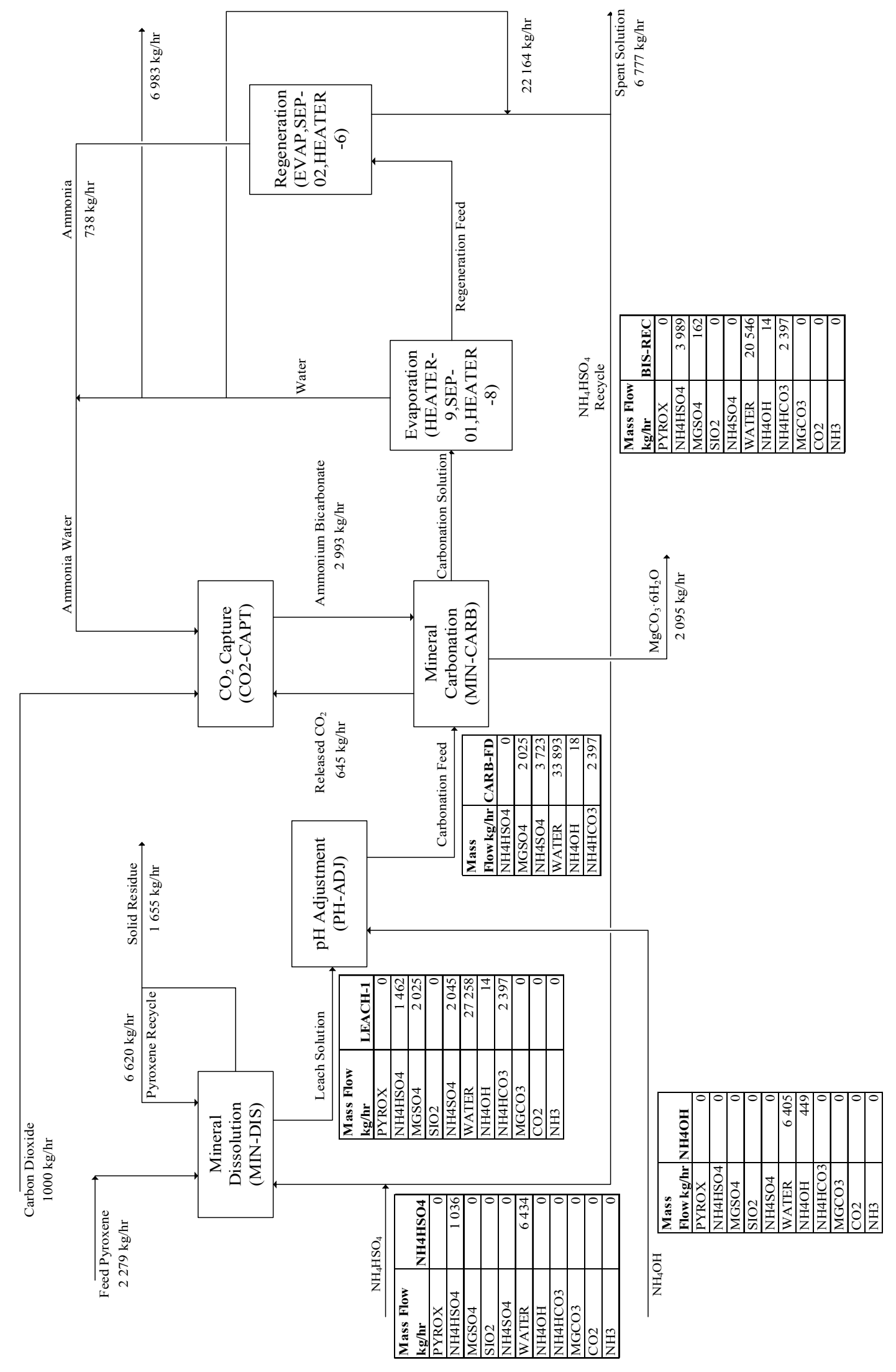


The carbonation reaction produces solid hydromagnesite $(2095 \mathrm{~kg} / \mathrm{hr})$ from the reaction of magnesium sulphate and ammonium bicarbonate. This reaction also produces $\mathrm{CO}_{2}$ as a biproduct, which is undesired since the process is designed to sequester carbon dioxide. To resolve this a closed $\mathrm{CO}_{2}$-loop was implemented by sending this carbon dioxide $(\mathrm{CO} 2, \mathrm{CO} 2-$ streams) to the capture unit.

The second stream (referred to as the "Carbonation Solution" in Figure 4-1) carries a solution rich in ammonium sulphate which is produced during dissolution, $\mathrm{pH}$ adjustment and carbonation. This stream also carries large quantities of water that have to be evaporated prior to thermal decomposition to regenerate reagents. During evaporation the stream is heated up to $120^{\circ} \mathrm{C}$ (HEATER-9) to drive-off water as steam, leaving solid ammonium sulphate. Most of the water is condensed (HEATER-8) and used in the process mixing it with ammonia for $\mathrm{CO}_{2}$ capture and using it to dissolve regenerated ammonium bisulphate. About $7000 \mathrm{~kg} / \mathrm{hr}$ is removed from the system to reduce the need to evaporate water that is not essential to the process.

The stream ("Regeneration Feed") carries solid crystals of ammonium sulphate that are to be decomposed, thermally, to regenerate ammonia and ammonium bisulphate. This is achieved in the thermal decomposition reactor (EVAP) that operates at $300{ }^{\circ} \mathrm{C}$. This results in the production of $738 \mathrm{~kg} / \mathrm{hr}$ ammonia and $4986 \mathrm{~kg} / \mathrm{hr}$ ammonium bisulphate from $5724 \mathrm{~kg} / \mathrm{hr}$ ammonium sulphate. Prior to being separated (SEP-02), the product stream (DEC-PRD) is cooled to $90{ }^{\circ} \mathrm{C}$. The separator removes ammonia which is fed to the capture unit (CO2-CAPT), and the RECYCLE stream, which consists of regenerated ammonium bisulphate, is sent for mixing with water.

The ammonia is used in carbon dioxide capture whereas $80 \%$ of the ammonium bisulphate carrying stream (BIS-REC) is recycled and 20\% becoming spent solution. This removes excess water and reagents (like ammonium bicarbonate) as well as carrying small amounts of unreacted magnesium sulphate. A trade-off is made in the purging this quantity of reagent since increasing the recycle may reduce external material requirements, whilst potentially increasing recycle sizes and unit energy requirement.

The $\mathrm{CO}_{2}$ capture unit, operating at $10{ }^{\circ} \mathrm{C}$ and 1 atm, uses ammonia and water to capture $\mathrm{CO}_{2}$. Condensed water from the evaporation circuit is fed alongside regenerated ammonia to produce an aqueous stream that captures $1000 \mathrm{~kg} / \mathrm{hr}$ of feed carbon dioxide, plus the carbon dioxide 
produced by the carbonation reaction. This produces the ammonium bicarbonate stream that is used as the $\mathrm{CO}_{2}$-carrier in the carbonation reaction.

The overall material requirements of the ammonium salts process are presented in Table 4-2. These indicate the feed input or make-up for regenerated components that would need to be supplied to the process. Since the process was developed with multiple recycle streams for key reagents and pyroxene, the material requirements are expected to be less than the stoichiometric requirements. For example, from a stoichiometric point of view the feed requirements of ammonium bisulphate required to extract magnesium from the silicate mineral is twice the amount of pyroxene input. This could imply that at least $5225 \mathrm{~kg} / \mathrm{hr}$ of ammonium bisulphate fresh feed would be required instead of about $1100 \mathrm{~kg} / \mathrm{hr}$ fresh feed this process requires. This is a significant reduction in material requirements. It highlights the importance of recycle streams and regeneration in mineral carbonation operations like the ammonium salts process. The benefit from this would be a reduced environmental footprint associated with reagent procurement, and could additionally lower the raw material cost of operating the ammonium salts process.

Table 4-2: Material requirements for ammonium salts carbonation

\begin{tabular}{|l|r|r|r|}
\hline & \multicolumn{3}{|c|}{ Mass Flow (kg/hr) } \\
\hline Component & 30\% Extraction & 50\% Extraction & $90 \%$ Extraction \\
\hline Carbon Dioxide & 1000 & 1000 & 1000 \\
\hline Pyroxene & 2279 & 2279 & 2279 \\
\hline Ammonium Bisulphate & 1036 & 1036 & 1151 \\
\hline Ammonium Hydroxide & 526 & 280 & 210 \\
\hline Water & 13940 & 10437 & 10151 \\
\hline
\end{tabular}

\subsubsection{Process Energy Requirements}

Figure 4-2 indicates the energy requirements of major process units in the ammonium salts carbonation process. This figure shows exothermic unit operations (negative energy requirements) and endothermic unit operations (positive energy requirements). The sensitivity of energy requirements of process units to changes in extraction efficiency is also demonstrated. A majority of the unit operations in the ammonium salts process require or generate less than $10000 \mathrm{MJ} / \mathrm{hr}$. The mineral dissolution (MIN-DIS), $\mathrm{pH}$ adjustment (PH-ADJ) and $\mathrm{CO}_{2}$ capture (CO2-CAPT) units are the only non-heater units that release energy, whereas the carbonation (MIN-CARB) and thermal decomposition (EVAP) units require energy. However, two process units stand out as major energy consuming or generating (HEATER-8, 
HEATER-9). These are the condenser that cools steam released during evaporation and the evaporator that drives-off water to facilitate the crystallization before thermal decomposition of ammonium sulphate, respectively. The evaporator in particular requires at least ten times more energy than the second highest energy consuming unit. This is because of the large quantities of water that need to be evaporated, as indicated in Table 4-2 and the energy balance around this unit presented in Appendix C.

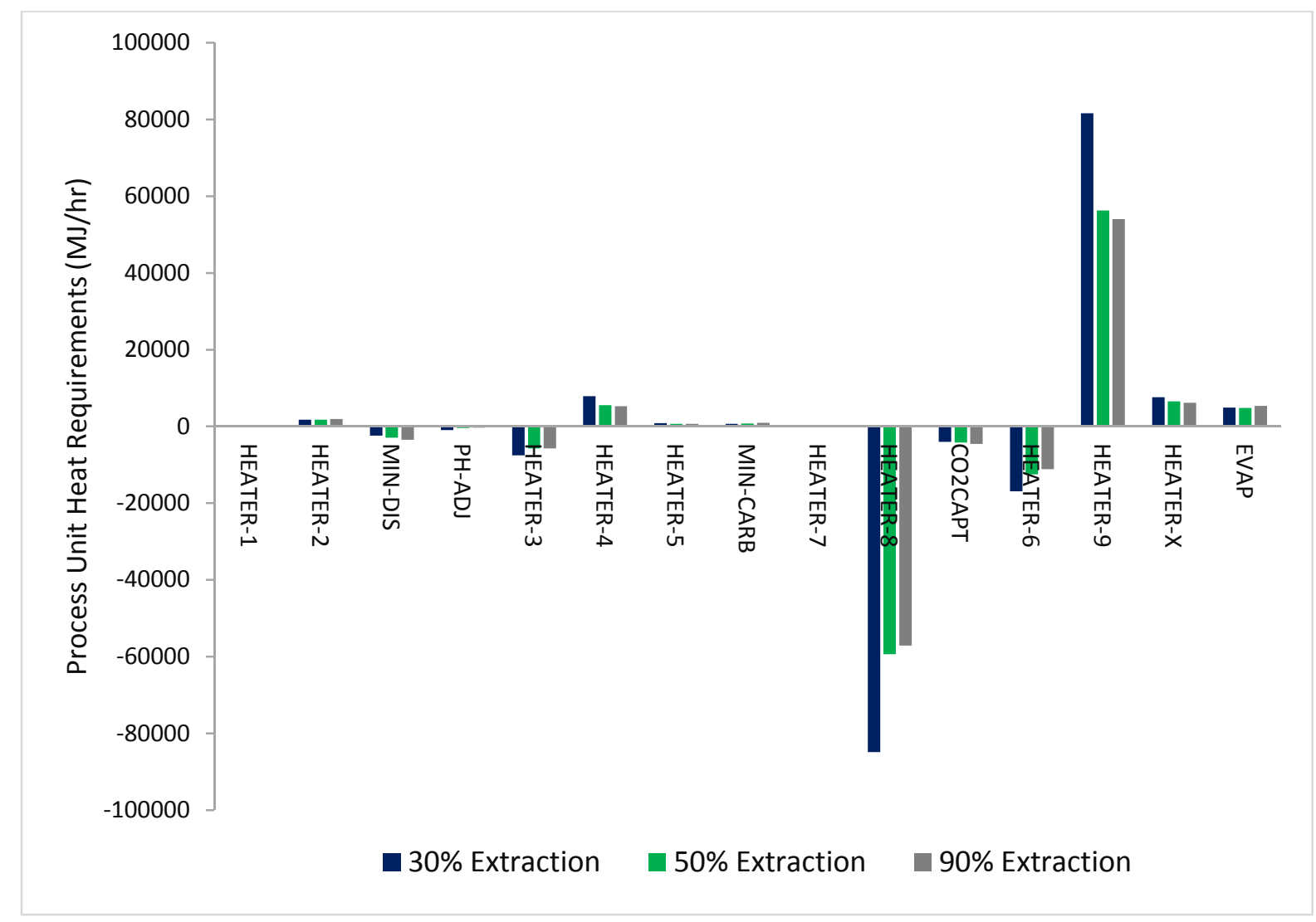

Figure 4-2: Energy requirements for major process units in the ammonium salts process

This observation is consistent with work carried out by Dri (2014) on the feasibility of the sequestration of steel plant $\mathrm{CO}_{2}$ emissions through the use of the ammonium salts process and steel slag. Though this study used a calcium-based feedstock, and HSC Chemistry Software (which does not have the ELECNRTL electrolyte property method for energy balances), the findings are qualitatively comparable. The authors found that the biggest energy demand was the evaporation of water prior to decomposition and that most key unit operations (mineral dissolution, $\mathrm{CO}_{2}$ capture, $\mathrm{pH}$ adjustment) were exothermic. However, they found carbonation to be slightly exothermic in comparison to slightly endothermic as indicated in Figure 4-2. This discrepancy could be a result of different carbon dioxide carriers used, since ammonium carbonate is used in the process studied by the authors instead of ammonium bicarbonate. The 
study by Dri (2014) is the only study known that evaluates the energy requirements of a process that implements the ammonium salts approach.

The total amount of energy required by the process ranges from about $75000 \mathrm{MJ} / \mathrm{hr}$ to 105000 $\mathrm{MJ} / \mathrm{hr}$ from the highest to lowest extraction efficiency. This is a significant amount of energy that could potentially result in substantial carbon dioxide penalties, as will be shown in Section 4.1.3. Identifying opportunities for heat integration appears to be a potential solution to reduce heat requirements, since the ammonium salts process also has a notable number of exothermic process units, for example HEATER-8, that release significant quantities of energy. Additionally some authors have suggested the use of a mechanical vapour recompression (MVR) evaporator that uses less energy than conventional evaporation (Dri, 2014).

It can also be noted that the amount of energy required for the process units generally decreases with increasing extraction efficiency. This is to be expected since an increase in extraction efficiency reduces the quantities of materials flowing through the units due to reduced sizes of recycle streams (Appendix B). This suggests that increasing the extraction efficiency could be beneficial towards reducing the overall heat requirements, as well as potentially reducing the size of process units due to the smaller recycle streams.

\subsubsection{Carbon Dioxide Footprint}

The material and energy requirements can be converted to process $\mathrm{CO}_{2}$ footprint through the use of SimaPro inventory analysis software. The inputs to this software are the material and energy requirements calculated from simulation through Aspen Plus. Table 4-3 indicates the individual contributions of process requirements to the overall carbon footprint of the ammonium salts process.

Table 4-3: Process contributions to carbon dioxide footprint $\left(\mathrm{kg}-\mathrm{CO}_{2} \mathrm{e}\right)$

\begin{tabular}{|l|r|r|r|}
\hline Description & 30\% Extraction & 50\% Extraction & 90\% Extraction \\
\hline Compression & 60 & 60 & 60 \\
\hline Heat Requirements & 7541 & 5484 & 5333 \\
\hline Ammonium Bisulphate & 695 & 695 & 772 \\
\hline Ammonium Hydroxide & 418 & 183 & 121 \\
\hline Process Water & 84 & 60 & 59 \\
\hline Total Footprint & 8798 & 6482 & 6346 \\
\hline
\end{tabular}

Figure 4-3 presents the carbon dioxide footprint of the ammonium salts process system. The results presented indicate a breakdown of the footprint, outlining the contributions of individual 
process inputs to the overall process footprint. Additionally, the effect of extraction efficiency in the dissolution stage on the carbon dioxide footprint is also presented.

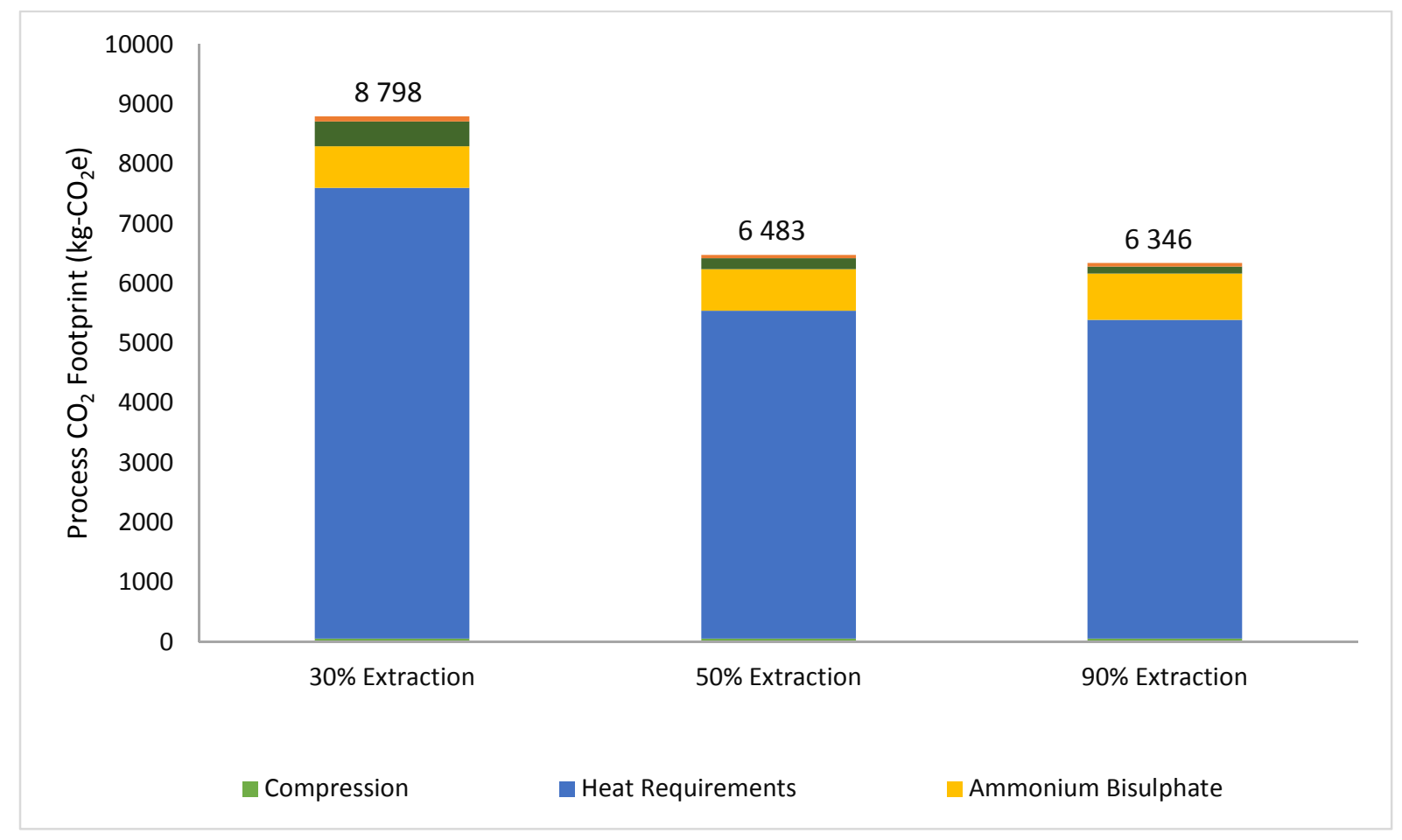

Figure 4-3: $\mathrm{CO}_{2}$ footprint of material and energy requirements of ammonium salts process $\left(\mathrm{kg}-\mathrm{CO}_{2} \mathrm{e}\right)$

It can be noted from this figure that the overall footprint well exceeds the $1000 \mathrm{~kg}$ of $\mathrm{CO}_{2}$ the process is designed to sequester, regardless of the extraction efficiency. The carbon dioxide footprint is more than 8 times greater than the carbon dioxide the process can sequester.

The most emissions intensive component of this mineral carbonation system is process heat generation. This accounts for about $85 \%$ of the total carbon dioxide emissions of the ammonium salts process. This observation has also been made by other authors (Dri, 2014, Sanna et al., 2014b) who have even suggested the implementation of a sodium salts-based process (Sanna et al., 2014c), of which the regeneration step is suggested to use a third of the energy in comparison to ammonium salts regeneration. However, the technical feasibility of the regeneration step is yet to be established, for the sodium salts-based process.

An option to consider could be exploring heat integration opportunities. Considering that the heat requirements will probably have to be reduced by at least $85 \%$, this implies that a similar percentage saving will be required from the heat exchanger network design. The heat exchanger network is unlikely to achieve this target due to thermodynamic limitations as well as heat losses. This suggests that heat integration in isolation is not enough to bring the process towards at the least carbon neutrality. The more reasonable approach would be to focus efforts 
on developing an alternate low-temperature regeneration process for this mineral carbonation system. (Dri, 2014) has also suggested that reducing the amount of water, thereby increasing the $\mathrm{S} / \mathrm{L}$ ratio, may reduce the energy requirements without affecting reaction performance. However, findings from another study have indicated that reducing the $\mathrm{S} / \mathrm{L}$ ratio may negatively affect extraction efficiency (Wang, 2011).

However, it must also be noted that the sum of the other contributions, excluding heat, also exceed $1000 \mathrm{~kg}-\mathrm{CO}_{2} \mathrm{e}$. This is primarily due to ammonium bisulphate and ammonium hydroxide production, to supply fresh feed to the process, which represent $695 \mathrm{~kg}-\mathrm{CO}_{2} \mathrm{e}$ and $418 \mathrm{~kg}-\mathrm{CO}_{2} \mathrm{e}$, respectively. The amount of fresh reagent feed can be lowered by increasing the recycle ratio, however this could potentially increase heat requirements and also present challenges with regards to process control since this can result in much greater "snowball effects" when disturbances occur (Svrcek et al., 2006).

The results in Figure 4-3 also indicate that the carbon dioxide footprint decreases with an increase in extraction efficiency. This decrease, though sizable, is not sufficient to reduce the carbon dioxide footprint below the $\mathrm{CO}_{2}$ threshold of $1000 \mathrm{~kg} / \mathrm{hr}$. This demonstrates that increasing extraction efficiency (which may require increasing reaction temperatures) does not yield enough of a benefit worth pursuing, in the current process configuration. Another potential carbon impact could be the ammonium bicarbonate leaving the process through the spent solution stream in Figure 4-1. As noted earlier (Section 4.1.1) and demonstrated in Table 4-1, this component is the carbon dioxide carrier in this process for carbonation, and thus would be expected to be carrying away some of the carbon dioxide that should have been sequestered. However, since post-processing of products is outside the scope of this study, this impact was not included.

\subsection{Lackner's HCl Multistage Process}

\subsubsection{Flowsheet Description and Mass Balance}

This process involves the extraction and conversion of magnesium in the silicate mineral to magnesium hydroxide through using hydrochloric acid as leaching agent, and multiple subsequent conversion steps. This process is based on a concept for a carbonation process proposed by Lackner et al. (1995). Experimental work related to this process was not conducted by the authors.

Similar to the ammonium salts process, simulations for this process were conducted for $30 \%$, $50 \%$ and $90 \%$ dissolution. A material balance for this process is presented in Figure $4-4$ for 
$30 \%$ extraction of magnesium ions from pyroxene and the chemical reactions occurring are presented in Table 4-4. Detailed mass balances and Aspen Plus simulations are presented in Appendix B and E, respectively.

Table 4-4: Chemical reactions occurring in Lackner's HCl multi-stage process

\begin{tabular}{|l|c|c|c|}
\hline Reactor Unit & Chemical Reaction & $\begin{array}{c}\text { T } \\
\left({ }^{\circ} \mathbf{C}\right)\end{array}$ & $\begin{array}{c}\text { P } \\
\text { (atm) }\end{array}$ \\
\hline $\begin{array}{l}\text { Mineral } \\
\text { Dissolution }\end{array}$ & $\mathrm{MgSiO}_{3}+\mathrm{HCl}+5 \mathrm{H}_{2} \mathrm{O} \rightarrow \mathrm{MgCl}_{2} \cdot 6 \mathrm{H}_{2} \mathrm{O}+\mathrm{SiO}_{2}$ & 70 & 1 \\
\hline Conversion & $\mathrm{MgCl}_{2} \cdot 6 \mathrm{H}_{2} \mathrm{O} \rightarrow \mathrm{MgOHCl}+\mathrm{HCl}+5 \mathrm{H}_{2} \mathrm{O}$ & 150 & 1 \\
\hline Repartition & $2 \mathrm{MgOHCl} \rightarrow \mathrm{Mg}(\mathrm{OH})_{2}+\mathrm{MgCl}_{2}$ & 25 & 1 \\
\hline $\begin{array}{l}\text { Mineral } \\
\text { Carbonation }\end{array}$ & $\mathrm{Mg}(\mathrm{OH})_{2}+\mathrm{CO}_{2} \rightarrow \mathrm{MgCO}_{3}+\mathrm{H}_{2} \mathrm{O}$ & 407 & 1 \\
\hline
\end{tabular}

The silicate mineral (pyroxene) and $\mathrm{HCl}$ solution are fed into preheaters where they are heated to $70{ }^{\circ} \mathrm{C}$, the dissolution reactor operating temperature. These are then fed into the reactor where magnesium is extracted from the silicate mineral matrix to produce $9247 \mathrm{~kg} / \mathrm{hr}$ of hydrated magnesium chloride $\left(\mathrm{MgCl}_{2} \cdot 6 \mathrm{H}_{2} \mathrm{O}\right)$.

The reaction products and unreacted reagents form a slurry that is then separated through solidliquid separations with unreacted solids ( $90 \%$ of solids stream) recycled back to the dissolution reactor (MIN-DIS), to improve the overall conversion of the silicate mineral, pyroxene. On the other hand, the separated solution is fed into the conversion process where $\mathrm{MgCl}_{2} \cdot 6 \mathrm{H}_{2} \mathrm{O}$ is converted to $3492 \mathrm{~kg} / \mathrm{hr} \mathrm{Mg}(\mathrm{OH}) \mathrm{Cl}$ at $150{ }^{\circ} \mathrm{C}$. This conversion process also regenerates acid when $\mathrm{MgCl}_{2} \cdot 6 \mathrm{H}_{2} \mathrm{O}$ initially loses the chemically bound water, with $\mathrm{HCl}$ eventually separating out instead of additional water release. A majority $(1514 \mathrm{~kg} / \mathrm{hr})$ of the regenerated acid is sent back to the mineral dissolution reactor as recycle. It is assumed that $10 \%$ removed accounts for losses and spent solution.

The $\mathrm{Mg}(\mathrm{OH}) \mathrm{Cl}$ produced is then fed into the repartitioning unit where, in the presence of water, the chemical is converted to produce $1326 \mathrm{~kg} / \mathrm{hr} \mathrm{Mg}(\mathrm{OH})_{2}$ and $2166 \mathrm{~kg} / \mathrm{hr} \mathrm{MgCl} 2$. The exiting magnesium chloride stream also serves as an outlet for water, which accumulates in the system. This avoids circulating large quantities of water which have no role in the process and could potentially increase unit capacities and heat requirements. The $\mathrm{Mg}(\mathrm{OH})_{2}$ produced is then carbonated with gaseous carbon dioxide to produce $1916 \mathrm{~kg} / \mathrm{hr}$ magnesium carbonate, at 407 ${ }^{\circ} \mathrm{C}$. 


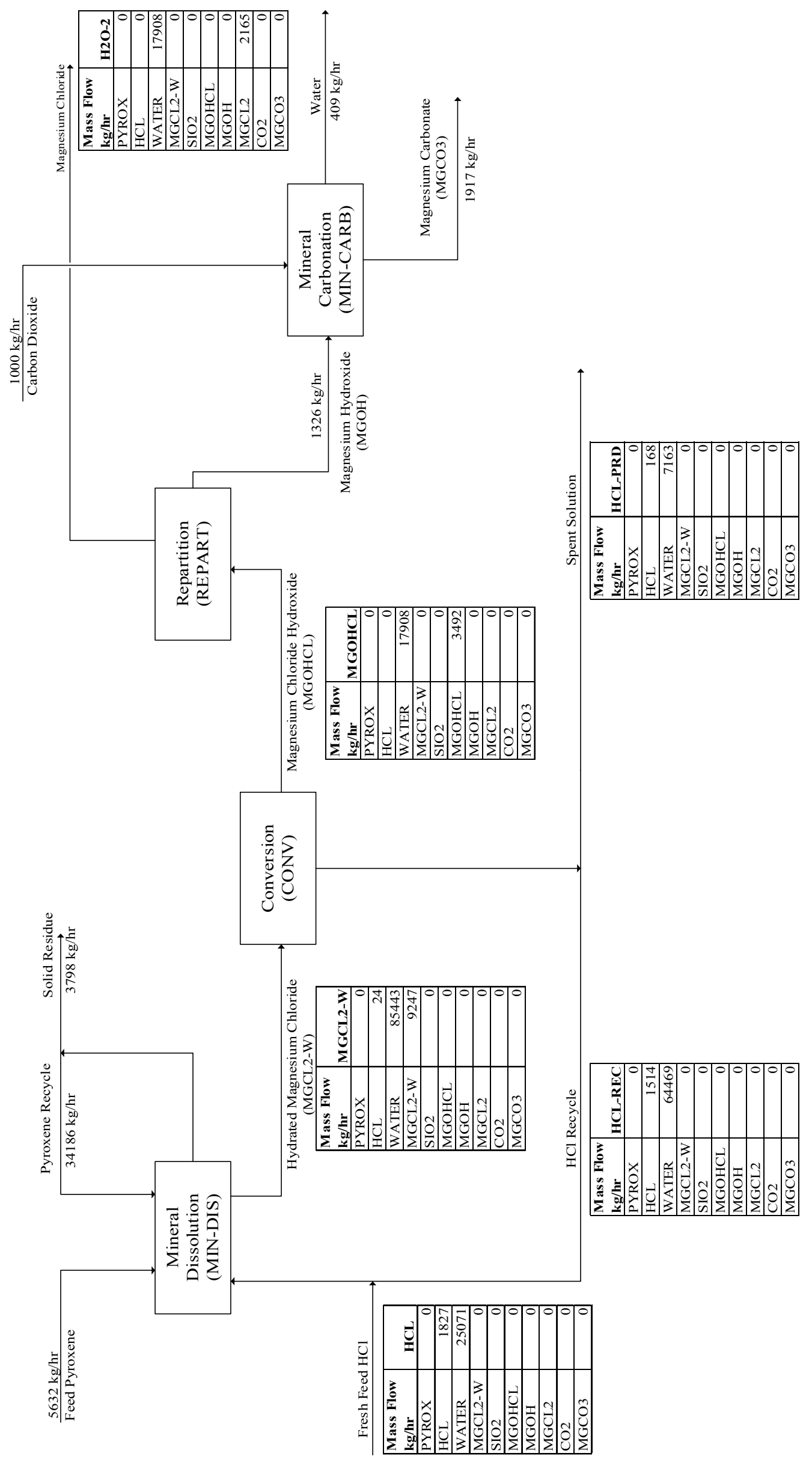


The overall material requirements for this process are presented in Table 4-5, and indicate that a substantial amount of pyroxene is required for the sequestration in this process, at about 5.6 times the carbon dioxide sequestered, by mass. This implies that larger size vessels would have to be designed to handle this material throughout the process. Similarly, recycle streams were implemented into this process which explain the lower fresh hydrochloric acid input, from a stoichiometry point of view. Since the design of the process is such that the feed into the reactor is sufficient from a stoichiometric perspective, through the use of recycle streams, the consequence is that as the extraction efficiency increases, the amount of fresh hydrochloric acid increases. Since the recycle ratio is kept constant in all simulations, the additional reagent for the increased conversion will have to come from increasing the fresh hydrochloric acid input.

Table 4-5: Material requirements for Lackner's HCl multi-stage process

\begin{tabular}{|l|r|r|r|}
\hline & \multicolumn{3}{|c|}{ Mass Flow (kg/hr) } \\
\hline Component & 30\% Extraction & 50\% Extraction & $90 \%$ Extraction \\
\hline Carbon Dioxide & 1000 & 1000 & 1000 \\
\hline Pyroxene & 5632 & 5632 & 5632 \\
\hline Hydrochloric Acid & 1827 & 2049 & 2228 \\
\hline Water & 25071 & 28124 & 30576 \\
\hline
\end{tabular}

\subsubsection{Process Energy Requirements}

The energy requirements of individual process units are presented in Figure 4-2. A majority of the unit operations (including mineral dissolution and carbonation) in this process require or release comparatively modest amounts of energy, with the exception of the conversion (CONV) and cooling (HEATER-4) process units. The conversion unit is the operation whereby high temperatures are used to drive off water and regenerate acid, in the process converting hydrated magnesium chloride to $\mathrm{Mg}(\mathrm{OH}) \mathrm{Cl}$. This process, carried out at $150{ }^{\circ} \mathrm{C}$, requires substantial amounts of energy to drive off large quantities of water. On the other hand, a notable amount of heat is released during cooling in the unit (HEATER-4). This unit condenses evaporated water and acid produced during the conversion process. The energy carried by the evaporated products could potentially be used to pre-heat prior to conversion, though it is anticipated that not all this energy will be recovered. The results obtained are not unexpected since findings made by Newall et al. (2000) pointed to evaporation as a major energy consumer. In fact, the authors established that the process energy requirements were four times that which is produced by the plant producing the emissions that the process was intended to sequester. 
The total amount of energy required rises from $230 \times 10^{3} \mathrm{MJ} / \mathrm{hr}$ to $280 \times 10^{3} \mathrm{MJ} / \mathrm{hr}$, whereas the energy released rises from $-183 \times 10^{3} \mathrm{MJ} / \mathrm{hr}$ to $-223 \times 10^{3} \mathrm{MJ} / \mathrm{hr}$ with increasing extraction efficiency.

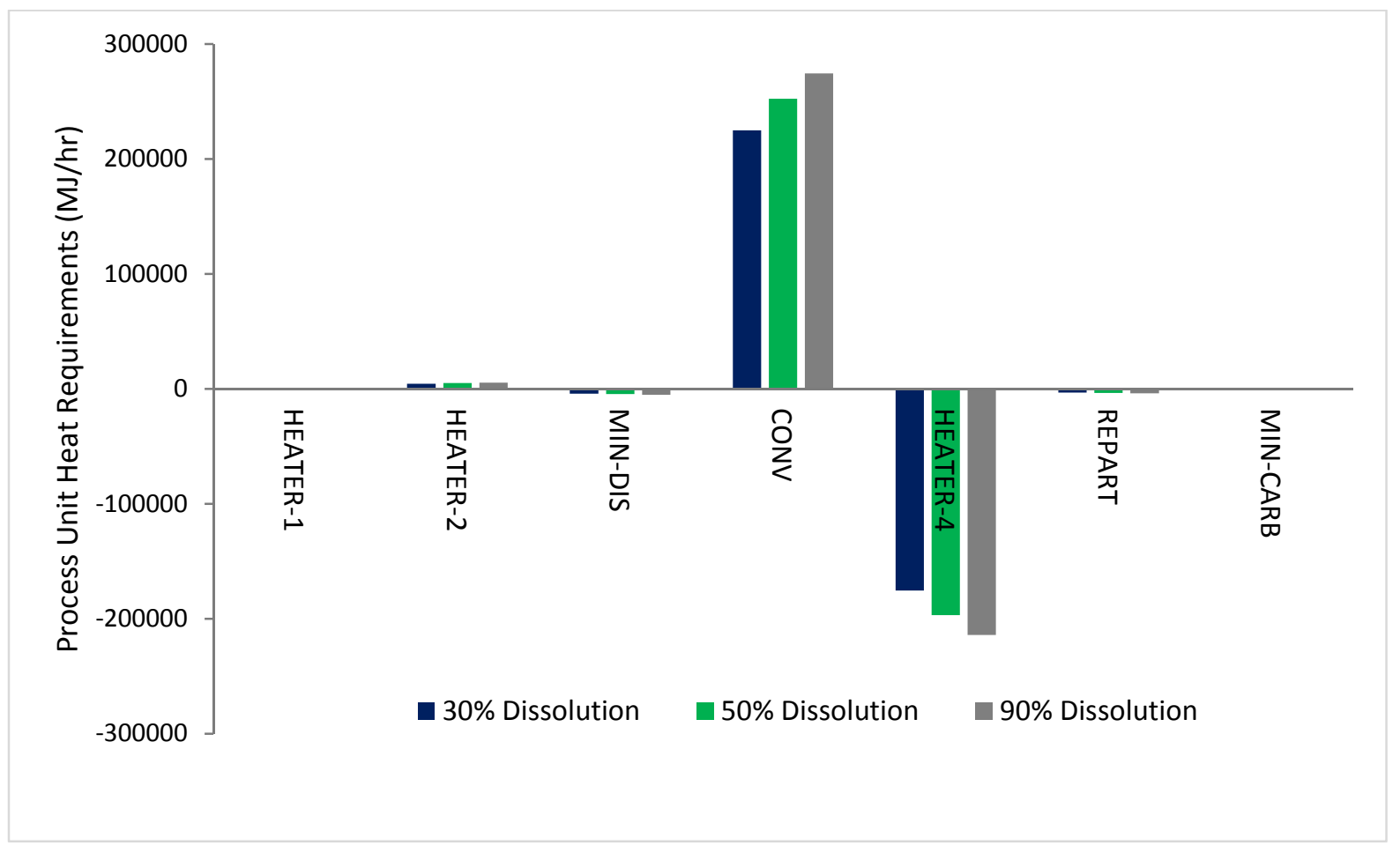

Figure 4-5: Energy requirements for major process units in the Lackner's HCl multi-stage process

An interesting observation is the increase in the energy requirements with increasing extraction efficiency. This can be attributed to the increase in fresh hydrochloric acid required with an increase in conversion. To illustrate this, we see a $12 \%$ increase in total energy requirements (from $230 \times 10^{3} \mathrm{MJ} / \mathrm{hr}$ to $258 \times 10^{3} \mathrm{MJ} / \mathrm{hr}$ ) with a $12 \%$ increase in hydrochloric acid requirement indicated in Table 4-5. The increase in fresh hydrochloric acid can be expected because the recycle ratio in all simulations is held constant, thus additional reagent to account for the increase in conversion will have to come from increasing the fresh reagent feed to meet stoichiometric requirements.

\subsubsection{Carbon Dioxide Footprint}

The energy and material requirements of the process translate to a carbon dioxide footprint associated with attaining these resources. The results presented in Table 4-6 and Figure 4-6 indicate a breakdown of contributions of different process inputs to the overall carbon footprint of the process. It also demonstrates the impact of increasing extraction efficiency on the carbon dioxide emissions attributed to the process. It is evident from the total footprint $(18.3-22.3$ 
ton- $\mathrm{CO}_{2} \mathrm{e}$ ) that the emissions released by the process are way above the emissions the process has potential to sequester ( 1 ton of $\mathrm{CO}_{2}$ ).

Table 4-6: Process contributions to carbon dioxide footprint ( $\left.\mathrm{kg}-\mathrm{CO}_{2} \mathrm{e}\right)$

\begin{tabular}{|l|r|r|r|}
\hline Description & 30\% Extraction & 50\% Extraction & 90\% Extraction \\
\hline Compression Electricity & 60 & 60 & 60 \\
\hline Heat Requirements & 15677 & 17587 & 19123 \\
\hline Hydrochloric Acid & 2394 & 2686 & 2920 \\
\hline Water & 163 & 183 & 199 \\
\hline Total Footprint & 18295 & 20516 & 22303 \\
\hline
\end{tabular}

About $98 \%$ of the total $\mathrm{CO}_{2}$ footprint for the process can be attributed to heat requirements and production of fresh hydrochloric acid feed. A significant portion of this can be ascribed to heat requirements, which account for $85.7 \%\left(15.7\right.$ ton- $\left.\mathrm{CO}_{2} \mathrm{e}\right)$ of the total carbon footprint when considering the base case. This footprint is linked to the large amounts of energy required in the conversion and regeneration stage, where water is evaporated, as discussed in Section 4.2.2. It is clear that without significant reduction of this component of the footprint the sustainability of this process, from a carbon balance perspective, in its current configuration is untenable.

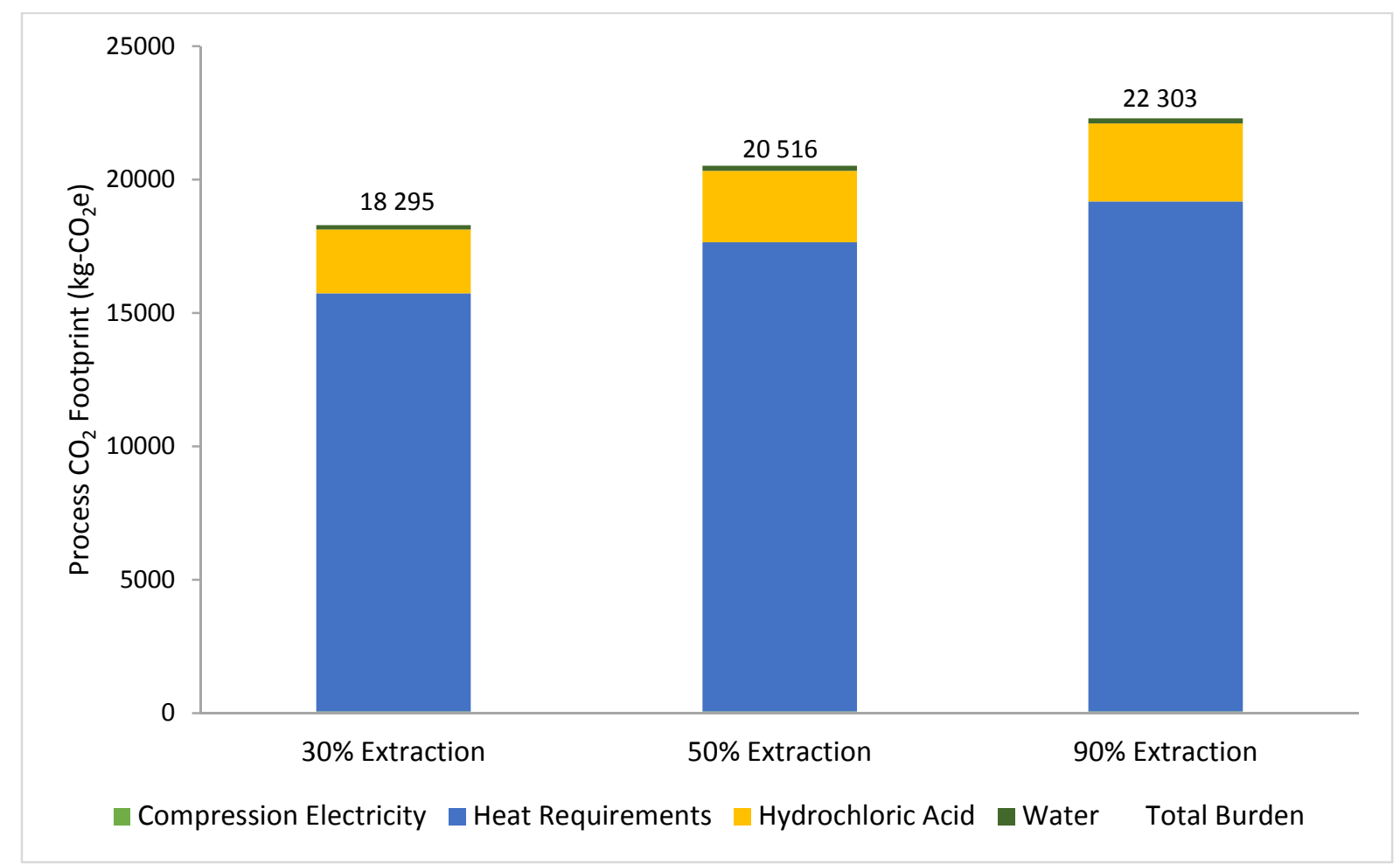

Figure 4-6: $\mathrm{CO}_{2}$ footprint of material and energy requirements of Lackner's HCl multi-stage process (kg-CO2e) 
Additionally, the second largest carbon dioxide emissions contribution, the production of fresh hydrochloric acid feed, is at least 2.4 ton- $\mathrm{CO}_{2}$ e for every 1 ton of carbon dioxide sequestered. This indicates that, in addition to heat requirements, the process also incurs prohibitive material requirements. The primary reason for this is the energy intensive operations that are used in the production of hydrochloric acid from hydrogen and chlorine. This means that an alternative source of hydrochloric acid may have to be considered, or a reduction in the amount of fresh feed required through increasing the recycle ratio, though this may impact plant sizing and process stability (Svrcek et al., 2006).

Similar to observations made in the discussion on process energy requirements (Section 4.2.2), the carbon dioxide footprint increases with increasing extraction efficiency. This can also be accounted for by the increase in fresh hydrochloric acid feed with increasing extraction efficiency because an equivalent percentage increase in the footprint is observed.

In order to bring the process towards carbon neutrality, a reduction in emissions of at least $95 \%$ would be required. Perhaps one would consider reducing these emissions through heat integration and mass balance optimisation (for example, using highly concentrated hydrochloric acid and/or increasing recycle ratios). This required reduction in emissions is quite considerable. It is greatly optimistic to expect that such emissions reduction targets may be achieved solely by this approach without significantly affecting the operability of the process.

\section{3 Åbo Akademi University (AAU) Process}

\subsubsection{Flowsheet Description and Mass Balance}

This is a multi-stage process that makes use of an ammonium salt (ammonium sulphate) to convert magnesium in the silicate mineral to the more reactive $\mathrm{Mg}(\mathrm{OH})_{2}$ compound through a series of unit operations. It is based on work conducted at the Åbo Akademi University (Fagerlund et al., 2010; Nduagu et al., 2012a/b; Nduagu, 2012). These authors have conducted experiments as well as evaluated the process from a life cycle perspective in a comparative study (Nduagu et al., 2012b).

The simulations for the $\AA A U$ process were developed for two different extraction and carbonation efficiencies that will be described as Scenario 1 and Scenario 2. These represent cases where, for Scenario 1, extraction efficiency is $66 \%$ and carbonation efficiency is $55 \%$ whereas for Scenario 2, the extraction efficiency is $100 \%$ and carbonation efficiency is $80 \%$. These scenarios are based on literature, where the maximum extraction and carbonation 
efficiencies currently obtainable are described by Scenario 1, and Scenario 2 describes conditions for a simulation developed at $\AA$ AU (Nduagu et al., 2012a).

The key chemical reactions occurring are presented in Table 4-7, and a material balance for this process presented in Figure 4-7 for Scenario 1. Detailed mass balances and Aspen Plus simulations are presented in Appendix B and E, respectively.

Table 4-7: Key reactions occurring in the Abo Akademi University process

\begin{tabular}{|l|c|c|c|}
\hline Reactor Unit & Chemical Reaction & $\begin{array}{c}\text { T } \\
\left({ }^{\circ} \mathbf{C}\right)\end{array}$ & $\begin{array}{c}\text { P } \\
\text { (atm) }\end{array}$ \\
\hline Mg-Extraction & $\mathrm{MgSiO}_{3}+\left(\mathrm{NH}_{4}\right)_{2} \mathrm{SO}_{4} \rightarrow \mathrm{MgSO}_{4}+\mathrm{SiO}_{2}+2 \mathrm{NH}_{3}+\mathrm{H}_{2} \mathrm{O}$ & 400 & 1 \\
\hline Precipitation & $\mathrm{MgSO}_{4}+2 \mathrm{NH}_{3}+2 \mathrm{H}_{2} \mathrm{O} \rightarrow \mathrm{Mg}(\mathrm{OH})_{2}+\left(\mathrm{NH}_{4}\right)_{2} \mathrm{SO}_{4}$ & 40 & 1 \\
\hline $\begin{array}{l}\text { Mineral } \\
\text { Carbonation }\end{array}$ & $\mathrm{Mg}(\mathrm{OH})_{2}+\mathrm{CO}_{2} \rightarrow \mathrm{MgCO}_{3}+\mathrm{H}_{2} \mathrm{O}$ & 450 & 19.74 \\
\hline
\end{tabular}

The silicate mineral feedstock, pyroxene, flowing at $2560 \mathrm{~kg} / \mathrm{hr}$ is preheated to reaction conditions $\left(400{ }^{\circ} \mathrm{C}, 1 \mathrm{~atm}\right)$ before being fed into the reactor (AS-REAC) where it reacts with ammonium sulphate. This reaction produces $2919 \mathrm{~kg} / \mathrm{hr}$ of magnesium sulphate $\left(\mathrm{MgSO}_{4}\right)$ alongside ammonia $\left(\mathrm{NH}_{3}\right)$, water $\left(\mathrm{H}_{2} \mathrm{O}\right)$ and silicon dioxide $\left(\mathrm{SiO}_{2}\right)$. The ammonia and water, produced in gaseous form, are separated from the solid product and cooled to $25{ }^{\circ} \mathrm{C}$. On the other hand the solids stream (SOLIDS) is also cooled.

The solids stream is subsequently mixed with $848 \mathrm{~kg} / \mathrm{hr}$ of water that dissolves $\mathrm{MgSO}_{4}$ produced in the extraction reactor (AS-REAC). This operation also allows for the solid-liquid separation of unreacted solids, that can then be recycled back to the extraction unit. The magnesium sulphate solution is then fed into a precipitation unit (PRECIP) that uses the cooled and condensed $\mathrm{NH}_{3}-\mathrm{H}_{2} \mathrm{O}$ stream to precipitate $1414 \mathrm{~kg} / \mathrm{hr}$ of $\mathrm{Mg}(\mathrm{OH})_{2}$, and at the same time regenerating ammonium sulphate (AS), at $40{ }^{\circ} \mathrm{C}$ and $1 \mathrm{~atm}$. This magnesium hydroxide is to be used in the carbonation unit whereas the regenerated sulphate salt (AS) will be used in the extraction unit. 


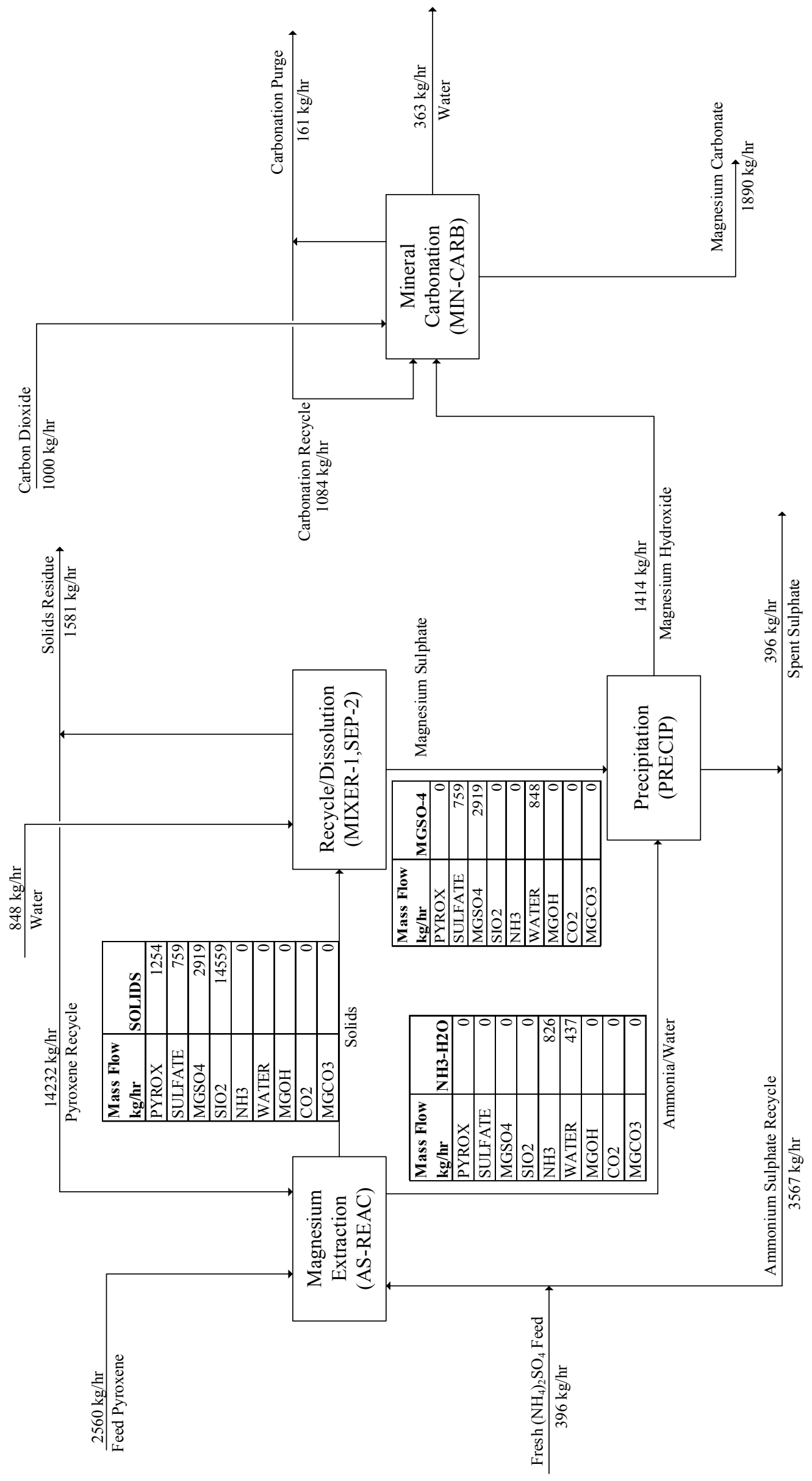


The precipitated $\operatorname{Mg}(\mathrm{OH})_{2}$ is then separated from the sulphate solution and preheated (HEATER-5) to carbonation conditions. It then enters the mineral carbonation unit (MINCARB) where it reacts with a feed of $1000 \mathrm{~kg} / \mathrm{hr} \mathrm{CO}$ at $450{ }^{\circ} \mathrm{C}$ and $20 \mathrm{bar}$. This unit produces a stable magnesium carbonate $\left(\mathrm{MgCO}_{3}\right)$ compound flowing at $1890 \mathrm{~kg} / \mathrm{hr}$, alongside water. These products are then separated to produce a pure $\mathrm{MgCO}_{3}$ stream that is cooled to $30{ }^{\circ} \mathrm{C}$ by the unit HEATER-2. After a series of separation steps, $90 \%$ of unreacted components are fed back into the mineral carbonation unit.

The AS solution produced by the precipitation unit is sent through a series of unit operations that remove water, such that the AS crystallises, making it suitable for recycle to the AS reactor. $90 \%$ of the stream $(3567 \mathrm{~kg} / \mathrm{hr}$ ) is fed back into the extraction unit whereas $10 \%$ is assumed to account for spent sulphate and losses. The use of recycle streams in this process increases the overall conversion from single pass, and optimises material use, in the process lowering fresh feed requirements, that cost money and carbon dioxide emissions associated with their production.

The process requires about 2.3 tons of pyroxene to sequester a ton of carbon dioxide, as indicated in Table 4-8. This process also requires relatively small amounts of ammonium sulphate fresh input. This is because of the use of recycle streams to feed the AS-REAC (see Appendix D) reactor with regenerated ammonium sulphate, as a result significantly reducing external reagent requirements. This process requires notably smaller quantities of water in comparison to the aqueous-based extraction processes. This is because the extraction reaction using ammonium sulphate occurs in a gas-solid reaction rather than in aqueous medium.

Table 4-8: Material requirements for Åbo Akademi University process

\begin{tabular}{|l|r|r|}
\hline & \multicolumn{2}{|c|}{ Mass Flow (kg/hr) } \\
\hline Component & Scenario 1 & Scenario 2 \\
\hline Carbon Dioxide & 1000 & 1000 \\
\hline Ammonium Sulphate & 396 & 396 \\
\hline Pyroxene & 2560 & 2281 \\
\hline Water & 848 & 848 \\
\hline
\end{tabular}

\subsubsection{Process Energy Requirements}

The energy requirements of major unit operations involved in the $\AA$ AU process are presented in Figure 4-8. These results also show the impact of an improvement in efficiencies of the extraction and carbonation stages. The process has an even spread of energy consuming and 
releasing units, that generally are below $2000 \mathrm{MJ} / \mathrm{hr}$. Most of the unit operations are heaters which function in cooling or heating streams as they enter or leave reactors.

Despite most of the process units being relatively low energy consumers, the extraction unit (AS-REAC) stands out and requires significantly more energy supplied. This is a key unit, where the extraction of magnesium using ammonium sulphate is conducted at high temperatures $\left(400{ }^{\circ} \mathrm{C}\right)$ in a gas-solid reaction. This is expected since the reaction to extract magnesium from the silicate mineral in this manner, is endothermic. Authors who have conducted energy analyses of this process have also identified this unit operation as the most intensive from an energy perspective (Nduagu et al., 2012a). The total amount of energy required for this unit for Scenario 1 is about $11500 \mathrm{MJ} / \mathrm{hr}$. The total required by the process in Scenario 2 is about $6300 \mathrm{MJ} / \mathrm{hr}$, meaning more than $80 \%$ of the process energy requirement is attributed to the extraction unit.

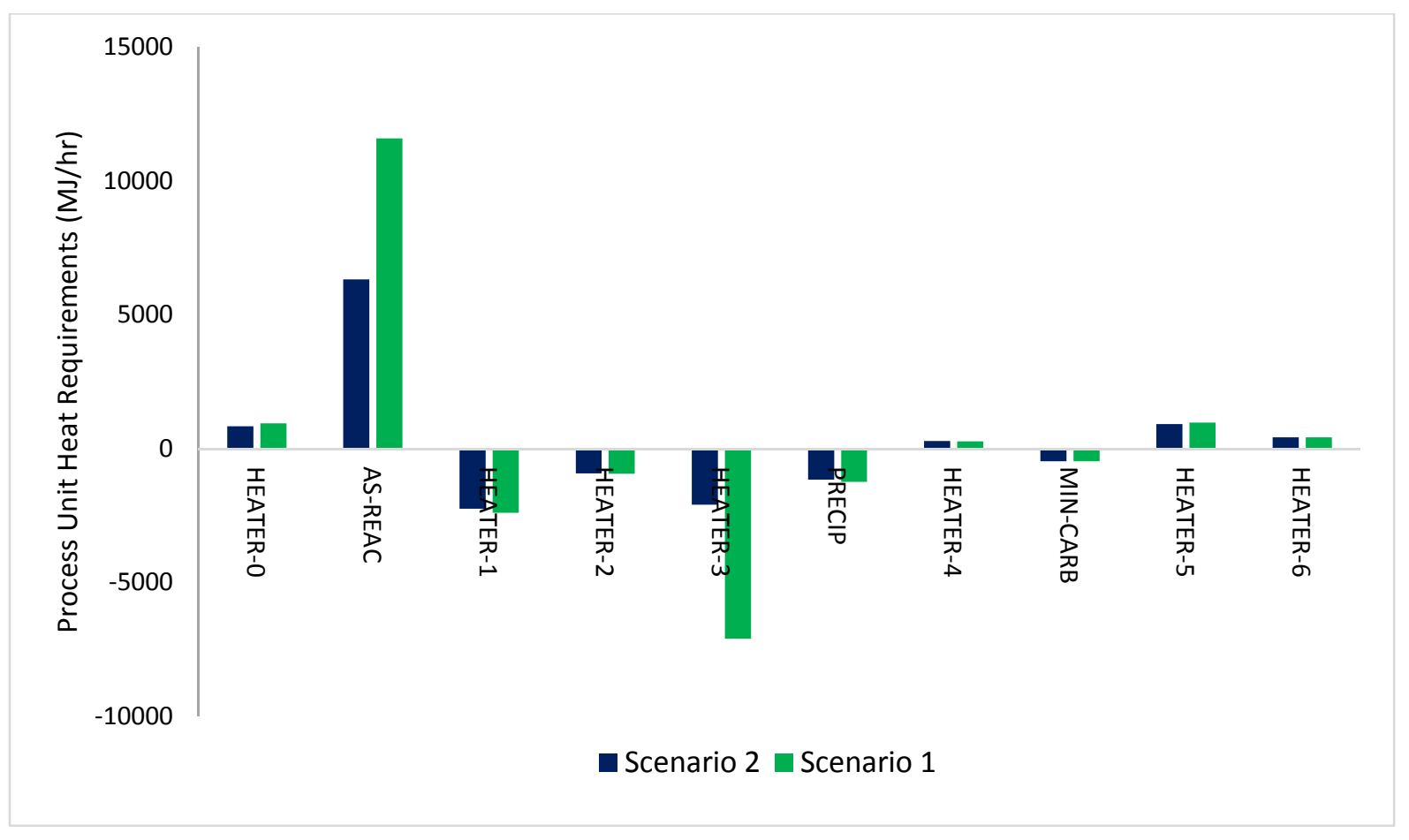

Figure 4-8: Energy requirements for major process units in the Åbo Akademi University process

It can be noted that the energy requirements of the majority of the process units are fairly unchanged with an increase in extraction and carbonation, aside from the extraction unit (ASREAC) and the solids stream cooler (HEATER-3). This is due to the absence of the solids recycle stream (PYR-REC) in Scenario 2, where 100\% of the pyroxene is converted to magnesium sulphate. As a result the solids product is just silicon dioxide, which is removed from the process. On the other hand at $66 \%$ extraction efficiency some unreacted pyroxene 
$(90 \%)$ is recycled. This recycle stream enters the reactor at lower temperatures than the reaction temperature thus requires additional heat to be brought up to the temperature at which the reaction occurs. This also explains the similarly marked difference in the energy released by the HEATER-3 process unit, which is the solids cooling unit.

\subsubsection{Carbon Dioxide Footprint}

Upon establishing material and energy demands of the process, carbon dioxide emissions associated with these demands can then be calculated. The contributions to the carbon dioxide footprint of process requirements are presented in Table 4-9 and Figure 4-9. The total footprints for the two scenarios (discussed in Section 4.3.1) are also presented.

Table 4-9: Process contributions to carbon dioxide footprint $\left(\mathrm{kg}-\mathrm{CO}_{2} \mathrm{e}\right)$

\begin{tabular}{|l|c|c|}
\hline Description & Scenario 1 & Scenario 2 \\
\hline Ammonium Sulphate & 232 & 232 \\
\hline Heat Requirements & 1019 & 634 \\
\hline Electricity Requirements & 84 & 84 \\
\hline Water & 6 & 6 \\
\hline $\mathrm{CO}_{2}$ Released & 13 & 24 \\
\hline Total Footprint & 1354 & 980 \\
\hline
\end{tabular}

It can be noted that the total footprint in Scenario 1 is slightly above $\left(1354 \mathrm{~kg}-\mathrm{CO}_{2} \mathrm{e}\right)$ the threshold of $1000 \mathrm{~kg}$ of carbon dioxide sequestered by the process whereas the footprint for Scenario 2 is slightly less than the threshold, in total $\left(980 \mathrm{~kg}-\mathrm{CO}_{2} \mathrm{e}\right)$. This is indicates a relatively promising start for this process, from a carbon dioxide emissions view point. This is because it is envisioned that additional optimisation and heat integration could reduce the footprint further, as has been indicated in work conducted by Nduagu et al. (2012b). Nonetheless, in its current configuration the process is not sustainable for Scenario 1 (which is based on experimental work) and marginally sustainable for Scenario 2 (based on, unlikely, complete extraction) from a carbon dioxide emissions perspective.

Further analysis into individual carbon footprint contributions indicates that heat requirements are the most significant, accounting for at least $65 \%$ of the emissions resulting from this process. This finding is consistent with observations made by Nduagu et al. (2012b), who also found heat input to be the largest emissions contribution. In fact, the largest two contributors (heat and fresh ammonium sulphate) account for almost $90 \%$ of the footprint. This intimates efforts to reduce the carbon footprint should be primarily focused on these two areas. 


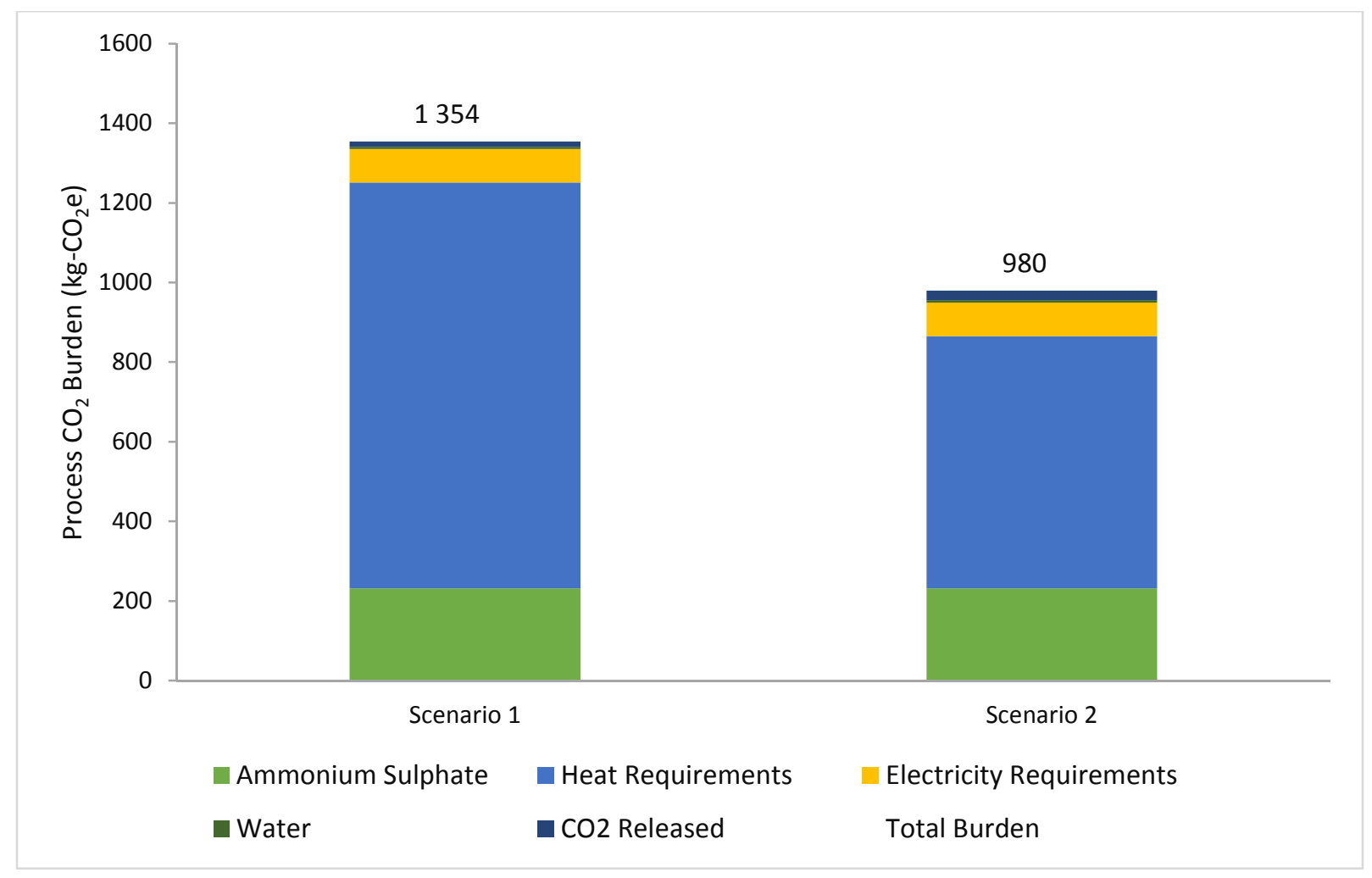

Figure 4-9: $\mathrm{CO}_{2}$ footprint of material and energy requirements of Abo Akademi University process (kg-CO2e)

\subsection{Mineral Acid pH-Swing Process}

\subsubsection{Flowsheet Description and Mass Balance}

The mineral acid $\mathrm{pH}$-swing process involves the extraction of magnesium from the silicate mineral (pyroxene) at low $\mathrm{pH}$ conditions, and the subsequent carbonation of the extracted cations at high $\mathrm{pH}$ or alkaline conditions. This process is based on the approach proposed by (Park et al., 2003) and experimental work conducted by (Vogeli, 2012; Meyer, 2014).

The simulation was run for three cases; based upon literature results (20\% dissolution, $65 \%$ carbonation), a theoretical increase to $30 \%$ dissolution and $90 \%$ carbonation, and a theoretical $50 \%$ dissolution and $90 \%$ carbonation. These will be referred to as base case, Scenario 1 and Scenario 2, respectively, in subsequent discussions.

A material balance for the process for the base case is presented in Figure 4-10 indicating key flows within the process. A more detailed mass balance and flowsheet is provided in Appendix $\mathrm{B}$ and $\mathrm{E}$ respectively. Additionally, the key chemical reactions occurring in this process are presented in Table 4-10. It must be noted that in the case of this particular process these chemical reactions have been developed for simplicity (since none have been proposed) and may not represent the exact path and reactions occurring in reality. 
Table 4-10: Key reactions occurring in the mineral acid pH-Swing process

\begin{tabular}{|l|c|c|c|}
\hline Reactor Unit & Chemical Reaction & $\begin{array}{c}\text { T } \\
\text { ( }\end{array}$ & $\begin{array}{c}\text { P } \\
\text { (atm) }\end{array}$ \\
\hline Mg-Extraction & $\mathrm{MgSiO}_{3}+2 \mathrm{HCl} \rightarrow \mathrm{MgCl}_{2}+\mathrm{SiO}_{2}+\mathrm{H}_{2} \mathrm{O}$ & 70 & 1 \\
\hline pH Adjustment & $\mathrm{NaOH}+\mathrm{HCl} \rightarrow \mathrm{NaCl}+\mathrm{H}_{2} \mathrm{O}$ & 25 & 1 \\
\hline $\begin{array}{l}\text { Mineral } \\
\text { Carbonation }\end{array}$ & $\mathrm{MgCl}_{2}+\mathrm{H}_{2} \mathrm{O}+\mathrm{CO}_{2} \rightarrow \mathrm{MgCO}_{3}+2 \mathrm{HCl}$ & 20 & 1 \\
\hline
\end{tabular}

This process uses hydrochloric acid $(\mathrm{HCl})$ to extract magnesium from pyroxene to produce $2222 \mathrm{~kg} / \mathrm{hr}$ of magnesium chloride in the dissolution reactor (MIN-DIS). These streams are both preheated to reaction conditions prior to being fed into the dissolution unit which operates at $70{ }^{\circ} \mathrm{C}$. Alongside magnesium chloride, the product also carries unreacted pyroxene in a slurry. These streams are separated through a solid-liquid separation, and $80 \%$ of the solids stream is recycled back into the dissolution reactor, to improve the overall conversion of pyroxene from the $20 \%$ single pass conversion.

The leach solution is then sent through a $\mathrm{pH}$ adjustment (PH-ADJ) process. Here unreacted hydrochloric acid is reacted with excess $(800 \mathrm{~kg} / \mathrm{hr})$ sodium hydroxide, to neutralize and adjust solution $\mathrm{pH}$ towards alkaline conditions. This is the step upon which the process is named, which provides the alkaline conditions that favour the carbonation reaction.

The $\mathrm{pH}$ adjusted leach solution (LEACH-2) is then fed to the mineral carbonation reactor (MIN-CARB) to react with $1000 \mathrm{~kg} / \mathrm{hr}$ of gaseous $\mathrm{CO}_{2}$. The product of this reaction is 1773 $\mathrm{kg} / \mathrm{hr}$ of solid magnesium carbonate. This reaction has been assumed to also regenerate hydrochloric acid, which can then be used in the dissolution circuit. About $90 \%$ of the regenerated acid is recycled back to the dissolution circuit, with the balance considered to be spent acid and losses. 


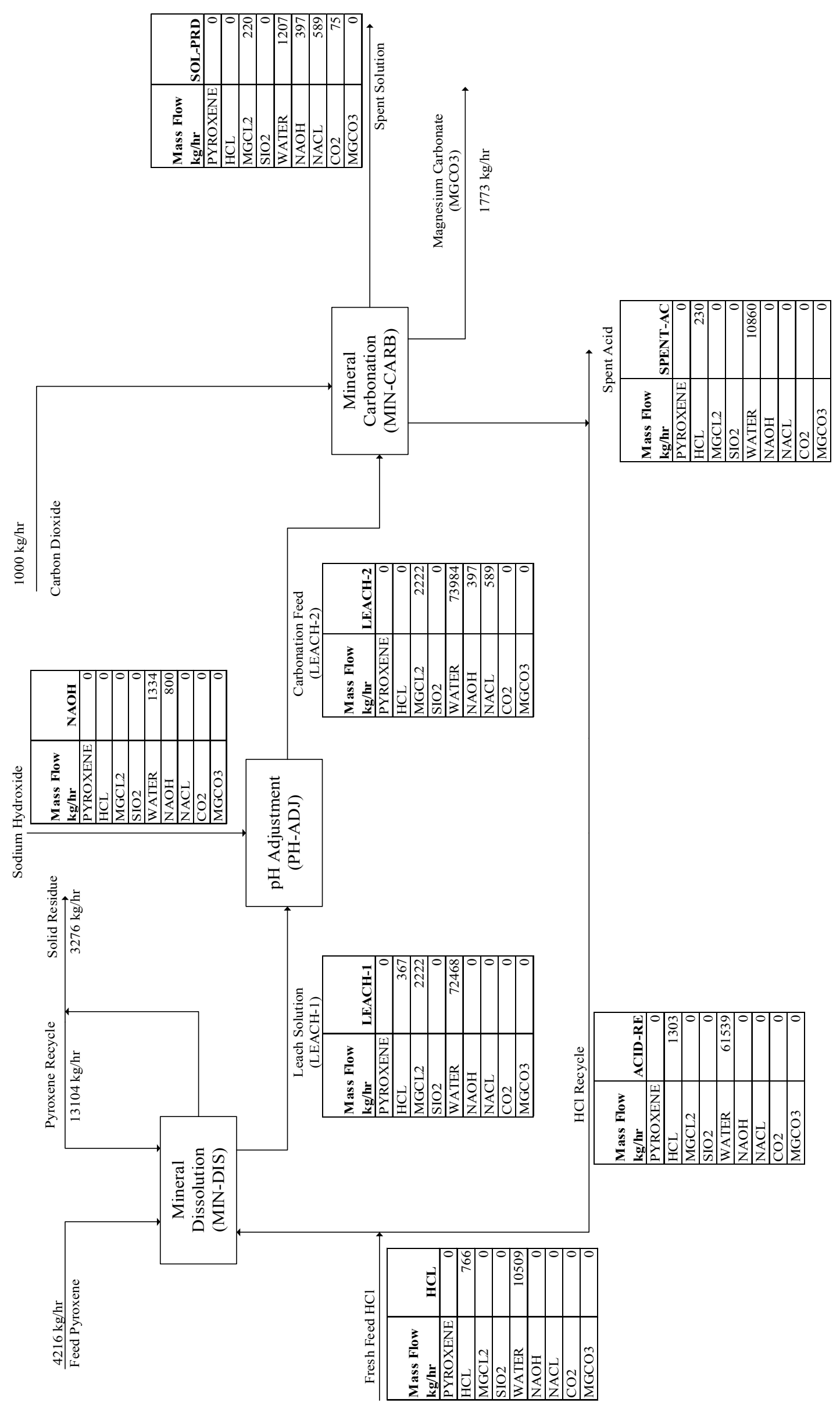

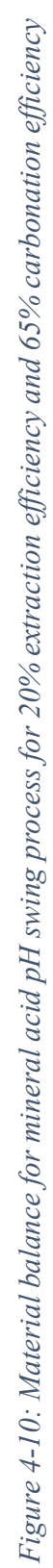


The overall input streams to this process are presented in Table 4-11 as well as the quantities that are required for each case. The tons of feed pyroxene required to sequester a ton of carbon dioxide can deduced from Table 4-11 to be about 4.2, which is relatively high. This implies that larger size vessels would have to be designed to handle this material throughout the process. It can also be seen that the amount of hydrochloric acid and water required increasing from the base case to through to Scenario 2. This can be attributed to the increase in extraction efficiency, which at constant pyroxene input rate would require more acid to react with the silicate mineral. The use of an acid recycle stream substantially reduces external $\mathrm{HCl}$ requirement, which would be significantly higher since at least twice the moles of $\mathrm{HCl}$ are required to react with $1 \mathrm{~mol}$ of pyroxene, from a stoichiometry standpoint.

Table 4-11: Material requirements for mineral acid pH swing process

\begin{tabular}{|l|c|c|c|}
\hline & \multicolumn{3}{|c|}{ Mass Flow (kg/hr) } \\
\hline Component & Base Case & Scenario 1 & Scenario 2 \\
\hline Carbon Dioxide & 1000 & 1000 & 1000 \\
\hline Pyroxene & 4216 & 4216 & 4216 \\
\hline Hydrochloric Acid & 766 & 1203 & 1677 \\
\hline Sodium Hydroxide & 800 & 800 & 800 \\
\hline Water & 5071 & 9825 & 16331 \\
\hline
\end{tabular}

\subsubsection{Process Energy Requirements}

The energy requirements of major unit operations are presented in Figure 4-11. This figure indicates that the units HEATER-1, HEATER-2 and HEATER-3 require heat to be supplied. These units are, respectively, the preheaters for feed pyroxene, fresh feed hydrochloric acid and recycled acid being fed into the dissolution process. The acid recycle heater is the most demanding in terms of energy requirements, requiring up to $26200 \mathrm{MJ} / \mathrm{hr}$ (Scenario 2). This constitutes more than $80 \%$ of the total energy requirements since up to $30000 \mathrm{MJ} / \mathrm{hr}$ (Scenario 2 ) is required by the overall process.

It is worth noting that these requirements increase from the base case up to Scenario 2 . This is a consequence of the increase in extraction efficiency which, as stated in Section 4.4.1, requires increased quantities of reagent. This is because across all simulations the recycle ratios were kept constant, thus any additional reagent would have to be sourced from an increased fresh feed input. This can be demonstrated by the observation that the percentage increase in fresh hydrochloric acid feed is comparable to the percentage increase in total energy requirements. For example, the percentage increase in hydrochloric acid from the base case to scenario 1 is 
about $57 \%$ (from $766 \mathrm{~kg} / \mathrm{hr}$ to $1203 \mathrm{~kg} / \mathrm{hr}$ ) which is comparable to the corresponding increase in energy, which is about 52\% (from $14760 \mathrm{MJ} / \mathrm{hr}$ to $22380 \mathrm{MJ} / \mathrm{hr}$ ).

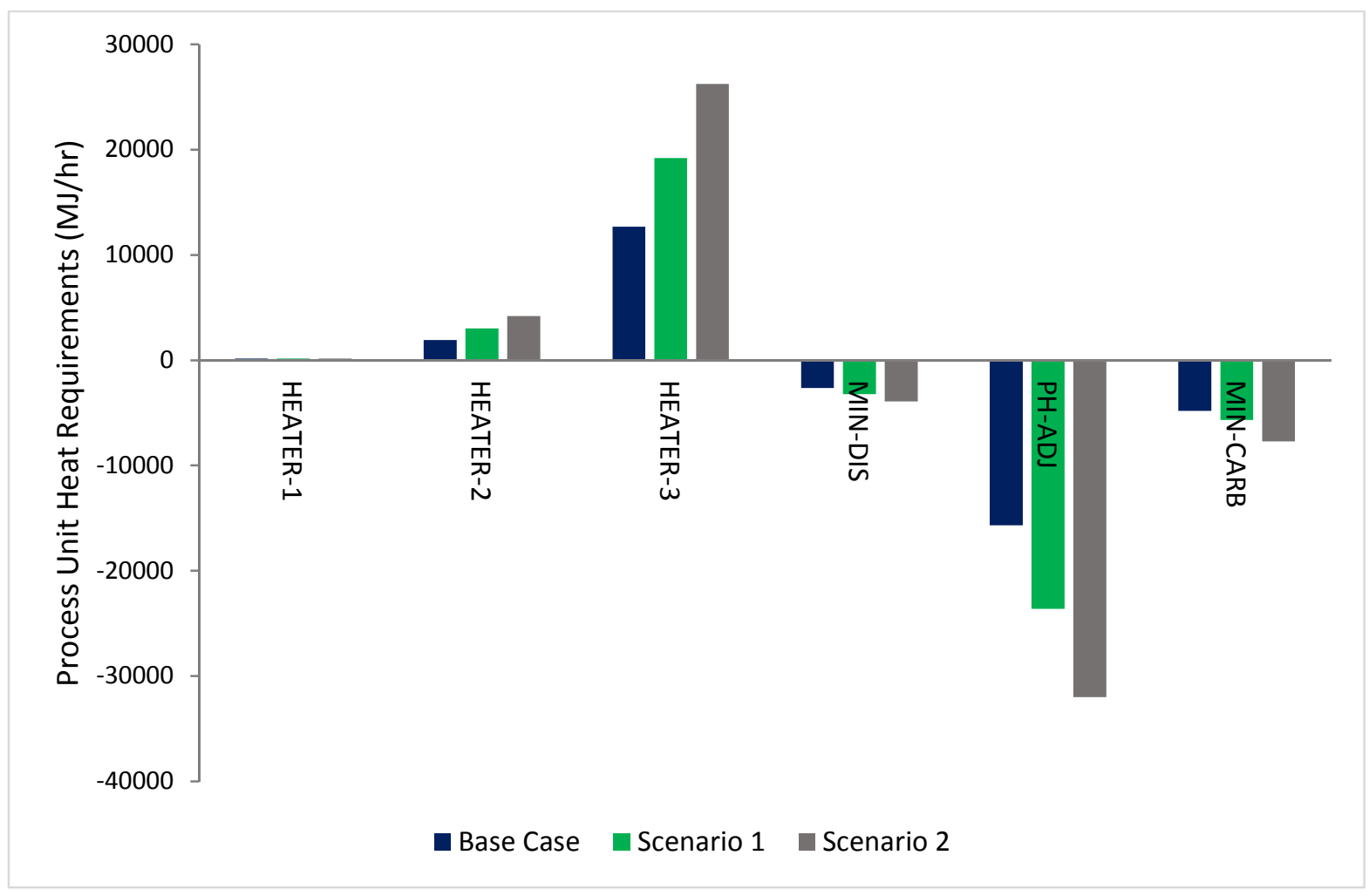

Figure 4-11: Energy requirements for major process units in the mineral acid pH swing process

It is also worth noting that there is an even split between energy releasing unit operations and those that require energy to be supplied. This could give an indication that heat integration may be worth exploring. In particular, the energy released during $\mathrm{pH}$ adjustment is comparable to the energy required by the acid recycle heater. However, to determine this additional analysis is required to establish the heat exchanger network that can be set up to achieve energy savings.

\subsubsection{Carbon Dioxide Footprint}

The material and energy requirements of the process ultimately translate to an environmental impact, in this case carbon dioxide equivalents, which is tabulated in Table 4-12 and presented in Figure 4-12. The contributions of process elements to the total carbon dioxide footprint of the process are indicated. Additionally, the impact of improvements in reaction efficiency on the carbon footprint is also demonstrated. 
Table 4-12: Process contributions to carbon dioxide footprint $\left(\mathrm{kg}-\mathrm{CO}_{2} \mathrm{e}\right)$

\begin{tabular}{|l|r|r|r|}
\hline Description & \multicolumn{1}{|c|}{ Base Case } & \multicolumn{1}{|c|}{ Scenario 1 } & Scenario 2 \\
\hline Compression & 60 & 60 & 60 \\
\hline Heat & 1033 & 1566 & 2143 \\
\hline Hydrochloric Acid & 1004 & 1577 & 2198 \\
\hline Sodium Hydroxide & 877 & 877 & 877 \\
\hline Water & 77 & 116 & 159 \\
\hline CO2 Released & 75 & 16 & 16 \\
\hline Total & 3126 & 4214 & 5454 \\
\hline
\end{tabular}

The carbon footprint of all three cases considered for this process is above the threshold of 1 $000 \mathrm{~kg}-\mathrm{CO}_{2} \mathrm{e}$. The total footprint for this process varies from $3126 \mathrm{~kg}-\mathrm{CO}_{2} \mathrm{e}$ to $5454 \mathrm{~kg}-\mathrm{CO}_{2} \mathrm{e}$ from the base case to Scenario 2. This implies that this process appears unsustainable from a carbon dioxide emissions point of view in its current configuration.

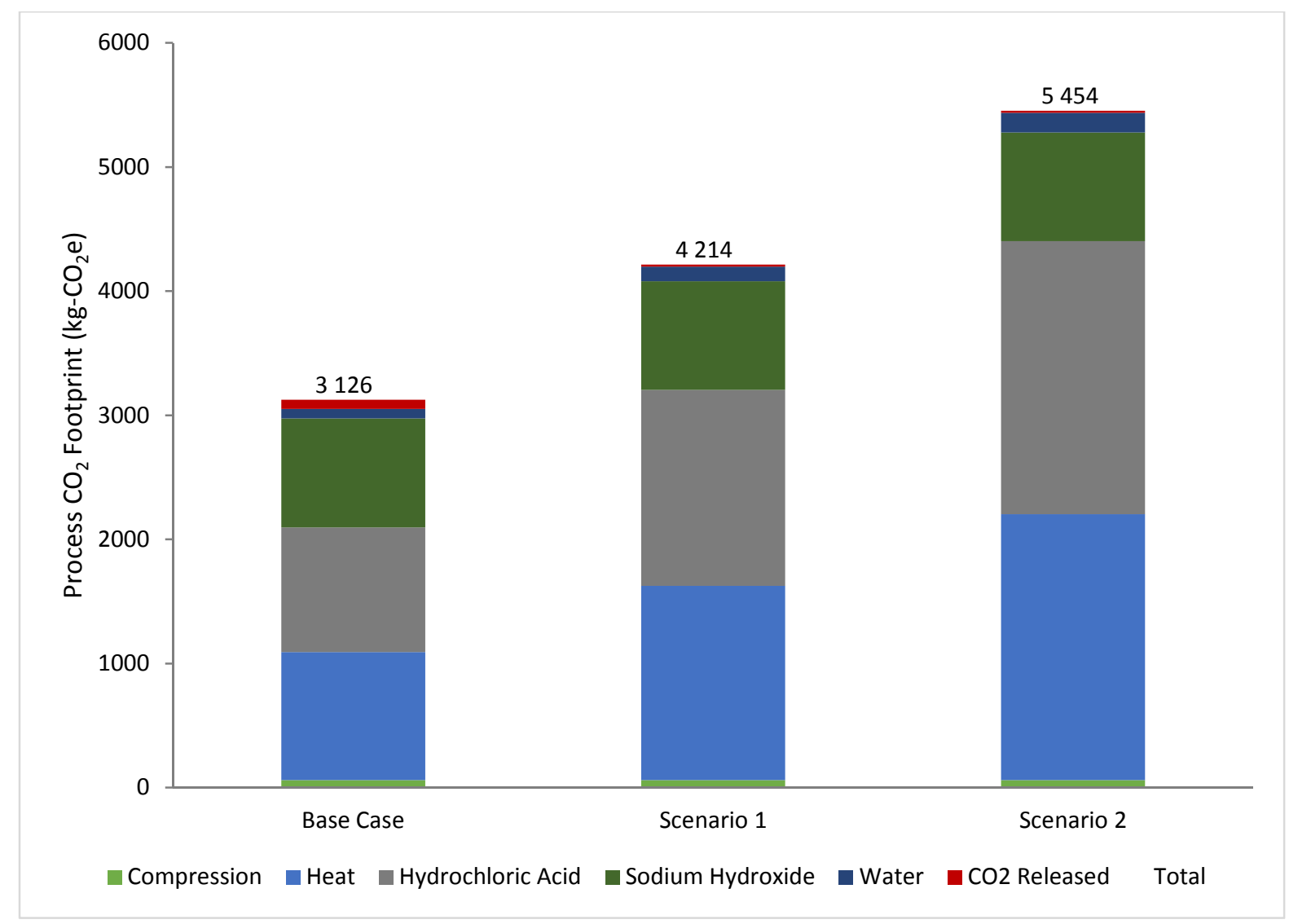

Figure 4-12: $\mathrm{CO}_{2}$ footprint of material and energy requirements of mineral acid pH swing process $\left(\mathrm{kg}-\mathrm{CO}_{2} \mathrm{e}\right)$

The major contributors to the footprint can be seen to be heat and fresh hydrochloric acid reagent requirements, with sodium hydroxide material requirements also contributing a significant portion to the carbon footprint. These account for between $77-89 \%$ of the footprint from the base case to Scenario 2. It is evident that these will have to be a focus for exploring 
reductions in order to significantly lower the carbon footprint of the process, regardless of extent of conversion of the silicate mineral.

The high $\mathrm{CO}_{2}$ footprint contribution for heat can be linked to the amount of heat required and the source through which this heat is obtained. Though the source, natural gas, has been identified as a cleaner fuel than coal or fuel oil, nonetheless the quantities required are substantial. Additionally, opportunities for energy integration appear to be limited. This represents a significant impediment to reduction of the contribution associated with heat generation, without exploring alternatives that are cleaner or using waste heat generated from other operations.

The carbon dioxide footprint associated with fresh hydrochloric acid is linked to the amount required as well as the means of production of the reagent. Incorporating recycle streams significantly reduces the amount of fresh acid required, from at least twice the amount of pyroxene to just above a third of the amount of pyroxene as discussed in Section 4.4.1. This may not be sufficient since the synthesis of hydrochloric acid results in about $1.3 \mathrm{~kg}-\mathrm{CO}_{2} \mathrm{e}$ for every kilogram of hydrochloric acid produced. Considering cleaner alternatives to the direct synthesis method may be useful.

Consistent with findings in the material and energy requirement results, the carbon dioxide footprint of the process increases from the base case through to Scenario 2, due to the explanation provided in Section 4.4.1. This is because for a constant emissions impact per unit requirement, the amount of material or energy required is directly proportional to the footprint accrued as a result of that requirement.

\subsection{Direct Aqueous Carbonation Process}

\subsubsection{Flowsheet Description and Mass Balance}

This is an aqueous carbonation process where the dissolution and carbonation steps occur in the same process step. It is considered the simplest approach, and makes use of carbonic acid produced through carbon dioxide dissolved in water as the extractive reagent. This process is based on work done at the National Energy Technology Laboratory (O'Connor et al., 2002; Gerdemann et al., 2007), though no work has been conducted with pyroxene as the silicate mineral feedstock.

The simulations for this process were run for the case where conversion was a modest $5 \%$, as well as a relatively higher $20 \%$ conversion. These conversions were selected as pyroxene is relatively less reactive, and lower conversions would be expected for the carbonation of this 
mineral than those provided in literature for serpentine and olivine, under industrial timescales. The chemical reactions occurring in the process are given in Table 4-13. It is assumed that the first reaction proceeds to completion, thus the conversions referred to are for the conversion of the silicate mineral to magnesium carbonate.

Table 4-13: Chemical reactions occurring in the direct aqueous carbonation process

\begin{tabular}{|l|c|c|c|}
\hline \multirow{2}{*}{ Reactor Unit } & Chemical Reaction & $\begin{array}{c}\mathbf{T} \\
\left({ }^{\circ} \mathbf{C}\right)\end{array}$ & $\begin{array}{c}\text { P } \\
(\mathbf{a t m})\end{array}$ \\
\hline \multirow{2}{*}{ Carbonation } & $\mathrm{H}_{2} \mathrm{O}+\mathrm{CO}_{2} \rightarrow \mathrm{H}_{2} \mathrm{CO}_{3}$ & 185 & 150 \\
\cline { 2 - 4 } & $\mathrm{MgSiO}_{3}+\mathrm{H}_{2} \mathrm{CO}_{3} \rightarrow \mathrm{MgCO}_{3}+\mathrm{SiO}_{2}+\mathrm{H}_{2} \mathrm{O}$ & 185 & 150 \\
\hline
\end{tabular}

The material balance for the direct aqueous carbonation process (for the case where $5 \%$ carbonation is achieved) is presented in Figure 4-13. This shows the key flows within the process that sequesters $1000 \mathrm{~kg} / \mathrm{hr}$ of carbon dioxide. A more detailed Aspen Plus simulation diagram is presented in Appendix E, with the corresponding material balance tables provided in Appendix B.

The direct aqueous process has been found to be enhanced by the addition of sodium bicarbonate and sodium chloride (O'Connor et al., 2000), thus the water feed stream carries about $2016 \mathrm{~kg} / \mathrm{hr}$ of ions from these components. This component flowrate corresponds to 966 $\mathrm{kg} / \mathrm{hr}$ sodium bicarbonate and $1050 \mathrm{~kg} / \mathrm{hr}$ sodium chloride mixed with the feed water. These amounts were determined based on the optimum compositions provided by Gerdemann et al. (2007), given in Table 2-3.

The water in this stream dissolves carbon dioxide producing carbonic acid, which facilitates the extraction of magnesium from pyroxene through the $\mathrm{H}+$ proton. It is assumed that the carbon dioxide completely dissolves in water, since the large amounts of water resulting in a $15 \%$ solids slurry (O'Connor et al., 2005) used are assumed to result in a solution below the saturation point at the reaction conditions. The bicarbonate then carbonates the magnesium precipitating $1883 \mathrm{~kg} / \mathrm{hr}$ solid magnesium carbonate.

Due to the low single pass conversion in the process, a large $43821 \mathrm{~kg} / \mathrm{hr}$ recycle stream is used to feed back into the carbonation reactor, unreacted pyroxene. This improves the process' overall conversion. This stream represents $85 \%$ of the solids leaving the reactor. To increase reagent usage, a large amount of the solution is also recycled ( $95 \%$ of liquids) back into the carbonation reactor. 


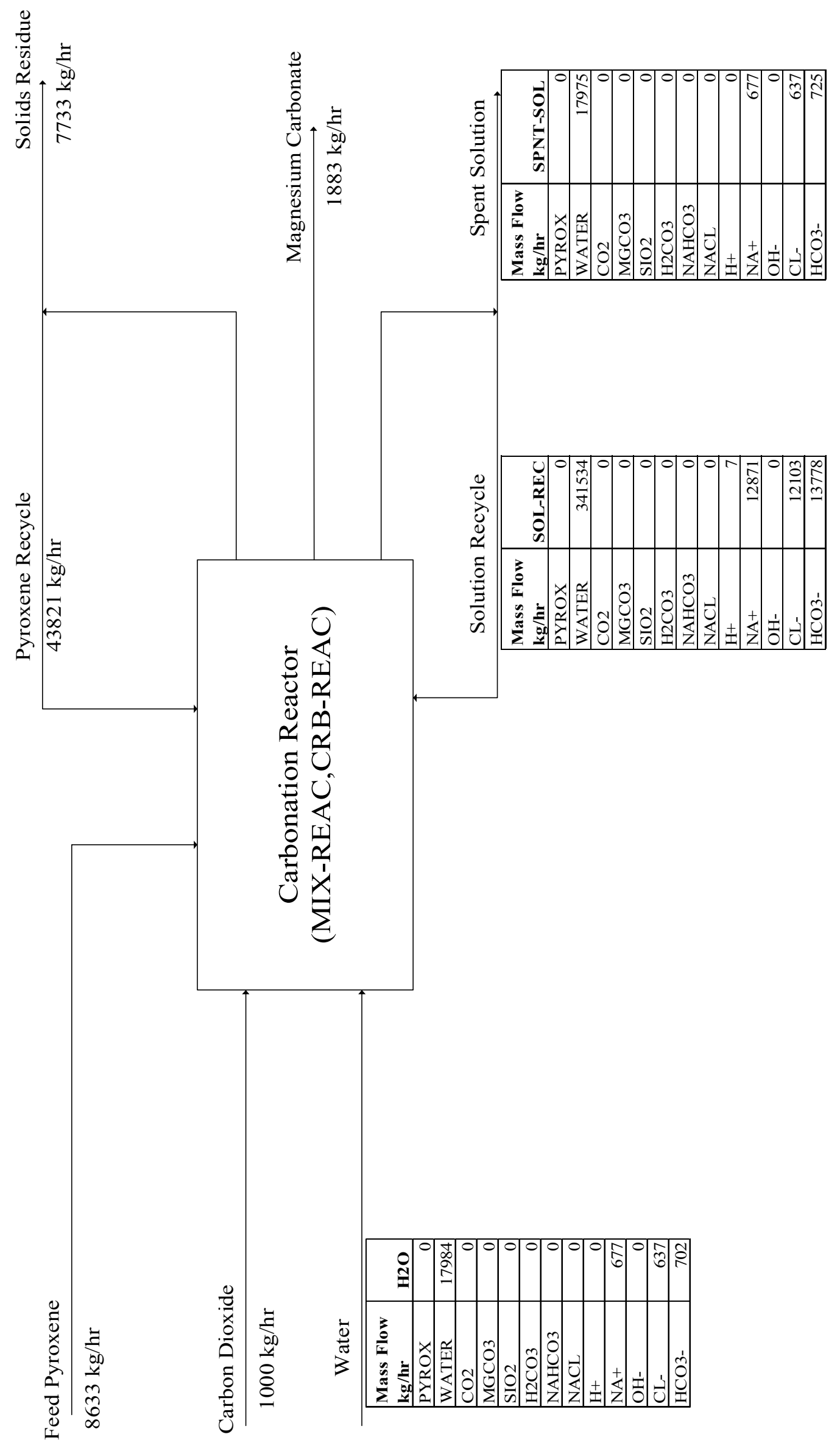


The input material requirements of the process are presented in Table 4-14. These show that significant quantities of pyroxene would be required to sequester a ton of carbon dioxide using this process. The quantity of feed pyroxene required per ton of carbon dioxide sequestered varies considerably for the two cases for which simulations were conducted, from about 8.6 in the case of $5 \%$ conversion to 3.5 when conversion increases to $20 \%$. The high value has implications that extend to influencing the size of units that will be required to process this material, as well as larger recycle streams.

Table 4-14: Material requirements for direct aqueous carbonation process

\begin{tabular}{|l|c|c|}
\hline & \multicolumn{2}{|c|}{ Mass Flow (kg/hr) } \\
\hline Component & 5\% Conversion & 20\% Conversion \\
\hline Carbon Dioxide & 1000 & 1000 \\
\hline Pyroxene & 8633 & 3514 \\
\hline Water & 17984 & 7819 \\
\hline Sodium Bicarbonate & 966 & 420 \\
\hline Sodium Chloride & 1050 & 468 \\
\hline
\end{tabular}

\subsubsection{Process Energy Requirements}

The energy requirements for most unit operations are relatively lower than the most intensive unit (HEATER-3) as shown in Figure 4-14. These units release or consume less than 2000 $\mathrm{MJ} / \mathrm{hr}$ in comparison to about $12500 \mathrm{MJ} / \mathrm{hr}$ required by HEATER-3. This unit is the feed water stream preheater that heats up water to $185^{\circ} \mathrm{C}$, the reaction conditions, prior to carbonation. This implies that even with $100 \%$ of the heat released by process units being used (which is improbable) it would be insufficient to supply the energy requirements for this unit. However, the use of the reactor exit stream to pre-heat the feed to the reactor could potentially significantly decrease this requirement, and has been suggested by some (Nduagu et al., 2012b; Giannoulakis et al., 2014).

The carbonation reactor was split into two reactor blocks (MIX-REAC, CRB-REAC) to separate the modelling of the dissolution of carbon dioxide in water and the carbonation reaction. This was done for computational simplicity during simulation development, since in reality these reactions occur in the same reactor. The formation of carbonic acid (MIX-REAC) and the carbonation reaction (CRB-REAC) are exothermic, however, release less than 2000 $\mathrm{MJ} / \mathrm{hr}$ combined. The production of carbonic acid was assumed to proceed to completion. 


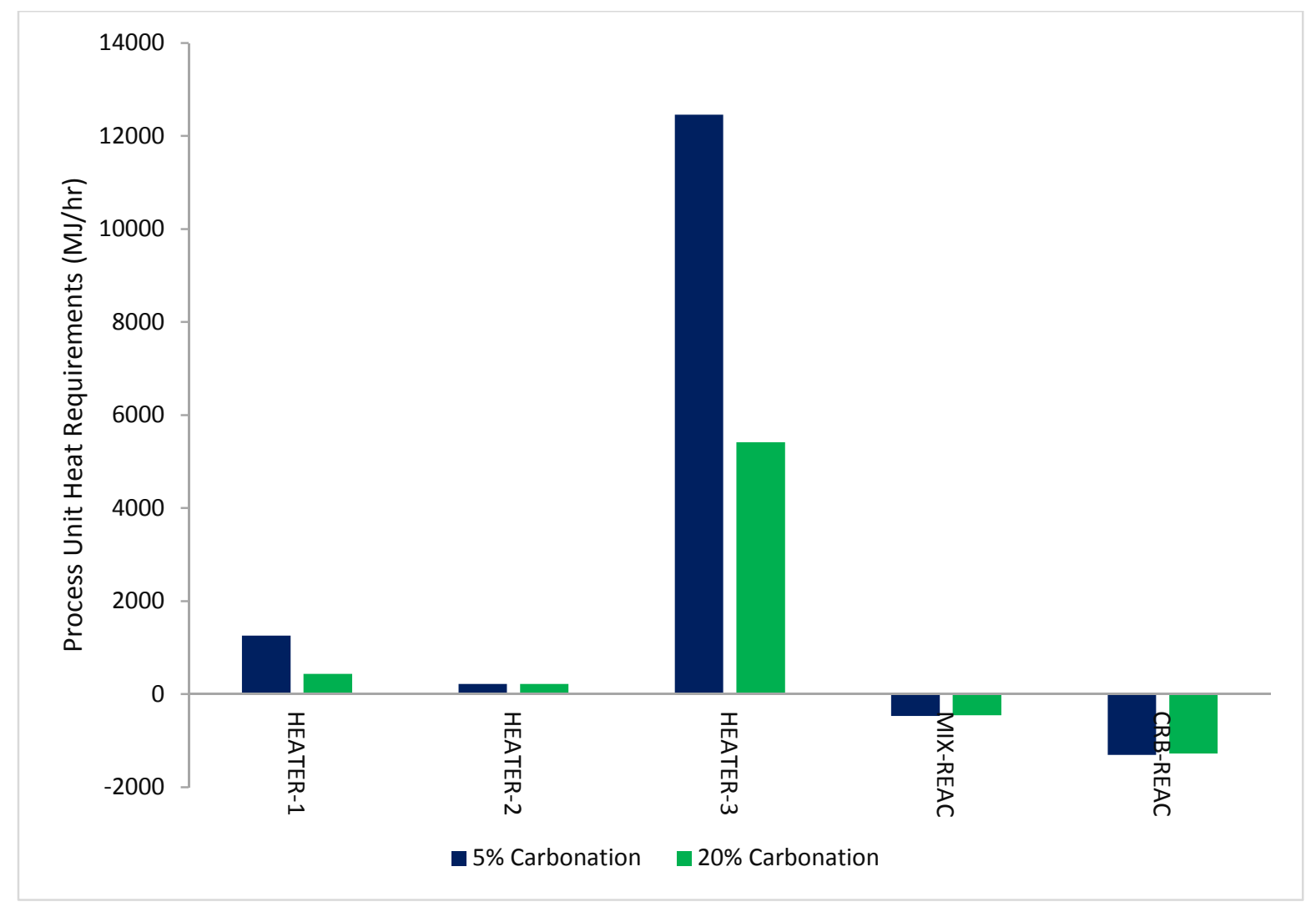

Figure 4-14: Energy requirements for major process units in the direct aqueous carbonation process

Figure 4-14 also shows that the energy requirement is fairly constant with an increase in conversion from $5 \%$ to $20 \%$, with the exception of HEATER-1. The decrease in requirement in HEATER-1 is linked to the decrease in pyroxene feedstock that requires preheating, whereas the decrease in requirement for HEATER-3 is linked to the decrease in make-up aqueous reagent. The amount of carbon dioxide fed into the process does not change, hence HEATER2 remains constant. This process also requires the compression of carbon dioxide to the reaction pressure of $150 \mathrm{~atm}$. This compression requires $50 \mathrm{MJ} / \mathrm{hr}$ of electrical energy, which is significantly lower in absolute terms in comparison to heat requirements.

\subsubsection{Carbon Dioxide Footprint}

The carbon dioxide emissions impacts of various process requirements are presented in Figure 4-15 and Table 4-15. The total emissions resulting from summing up the individual contributions of process requirements are also provided. The carbon dioxide emissions for both carbonation efficiencies simulated are above $1000 \mathrm{~kg}$ of $\mathrm{CO}_{2}$ sequestered, at $2364 \mathrm{~kg}-\mathrm{CO}_{2} \mathrm{e}$ for $5 \%$ carbonation and $1095 \mathrm{~kg}-\mathrm{CO}_{2}$ e for $20 \%$ carbonation. This suggests that the process cannot be considered sustainable from a carbon dioxide emissions perspective. 
Table 4-15: Process contributions to carbon dioxide footprint $\left(\mathrm{kg}-\mathrm{CO}_{2} \mathrm{e}\right)$

\begin{tabular}{|l|c|c|}
\hline Description & 5\% Carbonation & $\mathbf{2 0 \%}$ Carbonation \\
\hline Water & 117 & 51 \\
\hline Heat Requirements & 998 & 435 \\
\hline Electricity & 115 & 115 \\
\hline Sodium Bicarbonate & 1016 & 442 \\
\hline Sodium Chloride & 119 & 53 \\
\hline Total & 2364 & 1095 \\
\hline
\end{tabular}

These emissions are higher than those reported by Kichorfer et al. (2012) discussed in Section 2.2, albeit using different silicate minerals. However, it must be noted that the findings of those authors were based on general first-order level approximations of mass and energy requirements which could result in underestimation of the emissions contribution. In fact, the authors recommended the use of more rigorous and comprehensive modelling on simulation software like Aspen to better estimate reaction rates and energy requirements (Kirchofer et al., 2012). Additionally, the footprint associated with chemical reagent production was not included in accounting for emissions contributions.

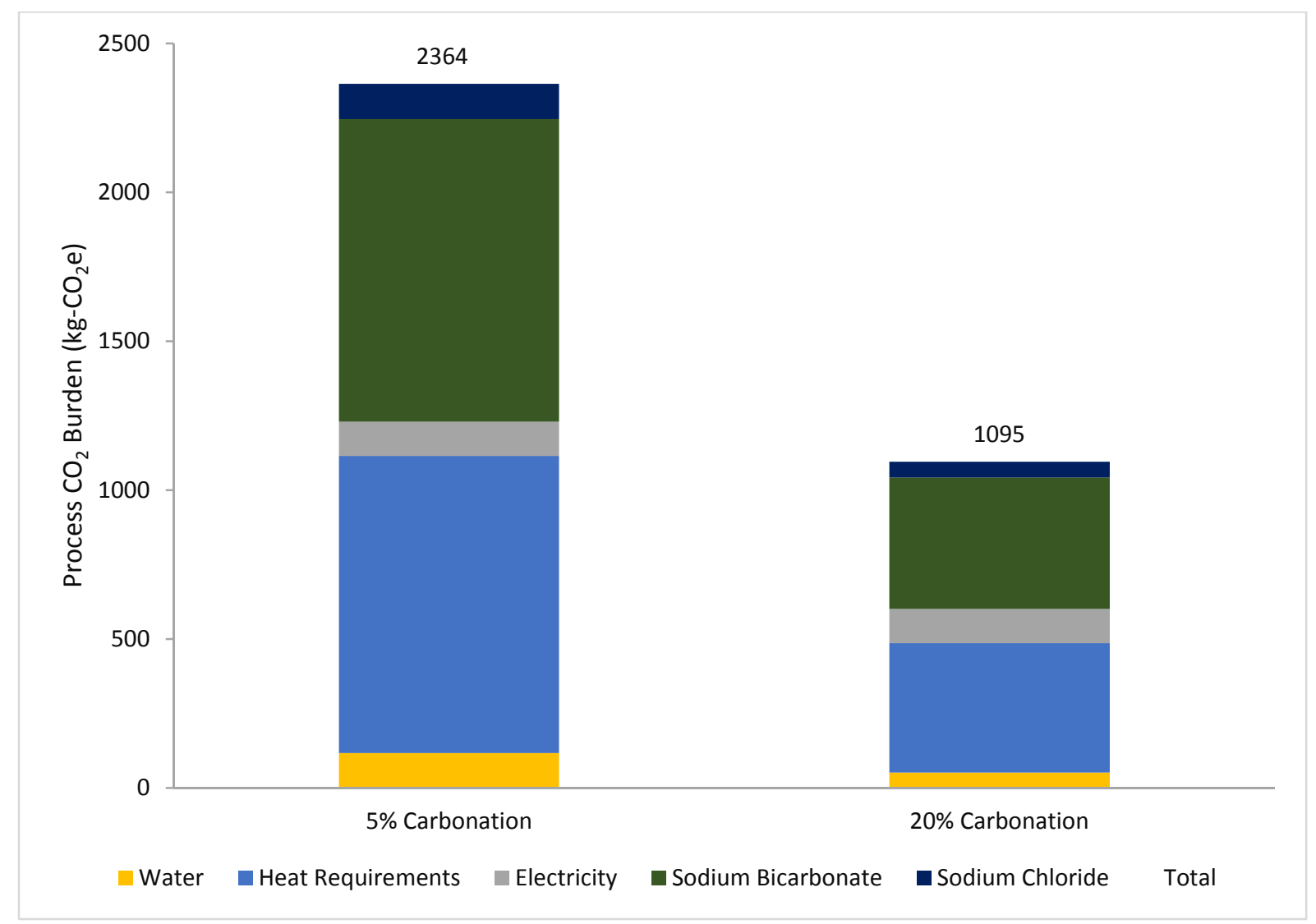

Figure 4-15: $\mathrm{CO}_{2}$ footprint of material and energy requirements of direct aqueous carbonation process (kg-CO $\mathrm{C}_{2}$ ) 
The results indicate that an increase in the carbonation efficiency from $5 \%$ to $20 \%$ could potentially result in the halving of the carbon footprint of the direct aqueous process. This brings the process closer to carbon neutrality, without the consideration of process improvements such as heat integration.

The primary contributors to the carbon dioxide footprint are heat requirements and the production of fresh sodium bicarbonate feed. These two account for $90 \%$ of the total emissions impact of the direct aqueous carbonation process. In this regard, focus to reduce overall emissions of the process should be focused on reducing these contributions. It is also worth noting that individually, the production of heat contributes about $1000 \mathrm{~kg}-\mathrm{CO}_{2} \mathrm{e}$, as well as the production of sodium bicarbonate. These, individually, push the process to the verge or just over carbon neutrality. This means that it is not enough to reduce one of these contributions in isolation, without achieving comparative reductions to the other. 


\section{Chapter 5}

\section{Discussion of Results}

The selected mineral carbonation processes result in varied material and energy requirements, and consequently varied carbon dioxide footprints. This section analyses and compares the processes from an energy and carbon dioxide footprint point of view, on the basis of the study results (Chapter 4). Also discussed are the potential effects of factors and variables associated with the key assumptions of the study. These effects pertain to the performance and viability of the proposed processes for the industrial-scale mineral carbonation of pyroxene-based feedstock, from both a technical and carbon neutrality perspective.

\subsection{Mineral Carbonation Process Energy Comparisons}

\subsubsection{General Comparisons}

Figure 5-1 indicates the total heat required by the individual processes to sequester a ton of carbon dioxide. This discussion focusses on process heat since it accounts for the majority of process energy requirements, which include electricity and heat.

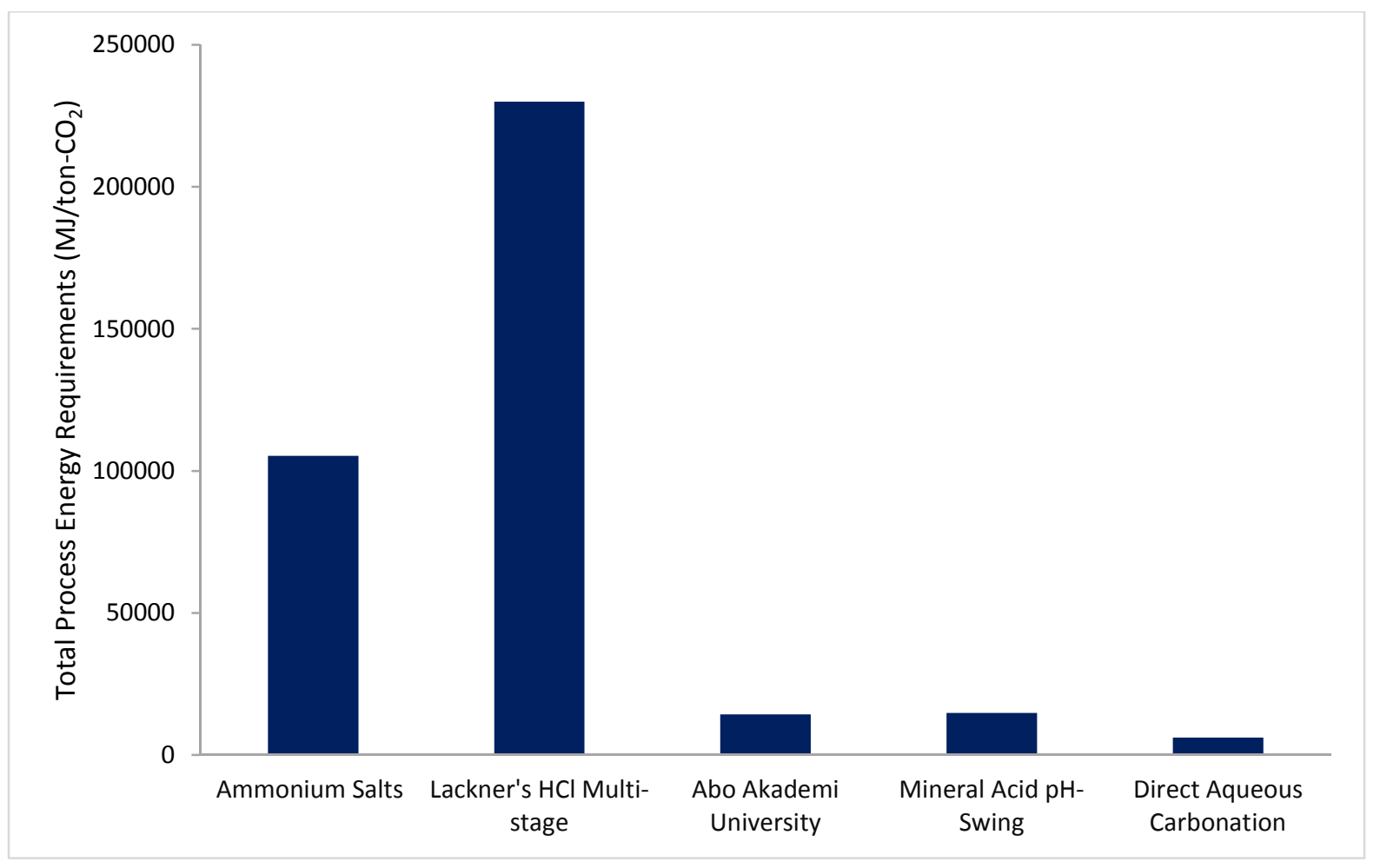

Figure 5-1: Comparison of process heat requirements of selected carbonation processes 
The results presented are based on the base case scenarios for all the processes, that is, based on the literature conversions or the minimum estimate made in absence of literature data. Lackner's $\mathrm{HCl}$ multi-stage process has the highest heat requirements of all the proposed processes with total heat requirements of $230 \times 10^{3} \mathrm{MJ} /$ ton- $\mathrm{CO}_{2}$ sequestered. This is followed by the ammonium salts process which requires just under half the heat at around $100 \times 10^{3}$ $\mathrm{MJ} /$ ton- $\mathrm{CO}_{2}$ sequestered. Comparatively, the last three processes require less than $30 \times 10^{3}$ $\mathrm{MJ} /$ ton- $\mathrm{CO}_{2}$ sequestered in total heat requirements. The process that requires the least heat is the direct aqueous process.

In general, it can be noted (Figure 5-1) that, with the exception of the direct aqueous process the aqueous processes have higher heat requirements when compared to the gas-solid Åbo Akademi University process. This is despite the high temperatures used in this process, particularly in the extraction and carbonation stages which use temperatures as high as $450{ }^{\circ} \mathrm{C}$ and pressures up to 20 bar. This temperature is higher than even the highest temperatures used in the aqueous processes $\left(300{ }^{\circ} \mathrm{C}\right.$ for thermal decomposition in the ammonium salts process). This appears somewhat counterintuitive considering that the expectation would be that higher temperatures and pressures result in higher heat requirements. This is not the case for the mineral carbonation processes considered.

This trend can be attributed to the strong direct link between heat consumption and the quantity of water that is used in the process. The Åbo Akademi University process requires just 848 $\mathrm{kg} / \mathrm{hr}$ of fresh feed water, whereas the aqueous processes generally require more than 10000 $\mathrm{kg} / \mathrm{hr}$ fresh feed water. Taking into account the recycle streams used in the selected processes, the water flows in the unit operations in the process are even larger than this feed input. With these large quantities of water requiring heating, the amount of heat required is increased substantially. Additionally, the evaporation of water in aqueous mineral carbonation processes is also a significant contributor to heat requirements. This involves a phase change which is accompanied by a latent heat of vaporisation that needs to be supplied, in addition to temperature-increasing heat, to convert the water into evaporated steam. It is thus not a coincidence that the two most intensive processes from a heat requirements perspective require the two largest water inputs (Table 5-1) and involve evaporation.

This observation is corroborated by work conducted by Dri et al. (2014), and noted by Sanna et al. (2014b), on the ammonium salts process, who noted that the heat associated with water evaporation in the ammonium salts process is excessive. In fact, in this process, the evaporation 
of water has higher energy requirements than the thermal decomposition of ammonium sulphate to regenerate ammonium bisulphate and ammonia (see Figure 4-2 in Section 4.1.2 in Chapter 4) which occurs at a much higher temperature of $300^{\circ} \mathrm{C}$. Similar concerns have been raised regarding Lackner's multi-stage $\mathrm{HCl}$ approach (Newall et al., 2000; Olajire, 2013). These findings do not imply that temperature is not an important variable in mineral carbonation operations, only that they draw attention to possibility that the presence and quantity of water in the process can influence the energy requirements much more than operating temperatures. This highlights an important shortcoming of the aqueous carbonation processes, with the large quantities of water used also having a negative effect on the footprint associated with heating requirements, and hence, sustainability of these processes.

\subsubsection{Comparisons between Aqueous Processes}

Amongst the aqueous carbonation processes the most energy intensive process is Lackner's multistage $\mathrm{HCl}$ process, whereas the direct aqueous process is the least intensive (Table 5-1).

Table 5-1: Comparison between feed water and process energy requirements of indirect aqueous processes

\begin{tabular}{|l|c|c|}
\hline \multicolumn{1}{|c|}{ Aqueous Carbonation Process } & $\begin{array}{c}\text { Fresh Water Feed } \\
(\mathbf{k g} / \mathbf{h r})\end{array}$ & $\begin{array}{c}\text { Process Energy Requirements } \\
\text { (MJ/hr) }\end{array}$ \\
\hline Direct Aqueous & 17984 & 6073 \\
\hline Lackner's Multi-Stage HCl & 25071 & 229962 \\
\hline Ammonium Salts & 12839 & 105349 \\
\hline Mineral Acid pH-Swing & 11843 & 14760 \\
\hline
\end{tabular}

Amongst the indirect aqueous processes, the link between the quantity of water and energy requirements is evident, with the process energy requirements increasing as the consumption of fresh water increases. Furthermore, the impact of phase change is also apparent in the much larger energy requirements of Lackner's multi-stage $\mathrm{HCl}$ process and the ammonium salts in comparison to the mineral acid $\mathrm{pH}$ swing process, which does not involve phase change. The However, the direct aqueous process, though using more water (18 $000 \mathrm{~kg} / \mathrm{hr})$, uses less energy than the ammonium salts $(13000 \mathrm{~kg} / \mathrm{hr})$ and the mineral acid pH swing (12 $000 \mathrm{~kg} / \mathrm{hr})$ indirect aqueous processes, as shown in Table 5-1. The comparison between direct aqueous and indirect aqueous processes appears to indicate another factor influencing the energy intensity of the aqueous carbonation routes, process simplicity. The direct aqueous process is a relatively simple process with one major reactor unit operating at a single temperature, whereas the indirect processes have multiple major reactor units operating at different temperatures, requiring intermediate heating which raises heat requirements. 
These observations suggest that for aqueous mineral carbonation processes to reduce some of their energy requirements, it may be advisable to avoid energy intensive unit operations that involve phase changes such as evaporation, in addition to minimising water usage.

\subsection{Mineral Carbonation Carbon Dioxide Footprint Comparisons}

\subsubsection{Comparisons of Overall $\mathrm{CO}_{2}$ Footprints}

A comparison of the carbon dioxide footprint of the selected mineral carbonation processes is presented in Figure 5-2, for the base case of all processes (as explained in Section 5.1.1). A tabulated version of this figure, providing more detail is presented in Appendix F:. These provide information on the total emissions impact of the process, as well as individual contributions of process elements to the footprint.

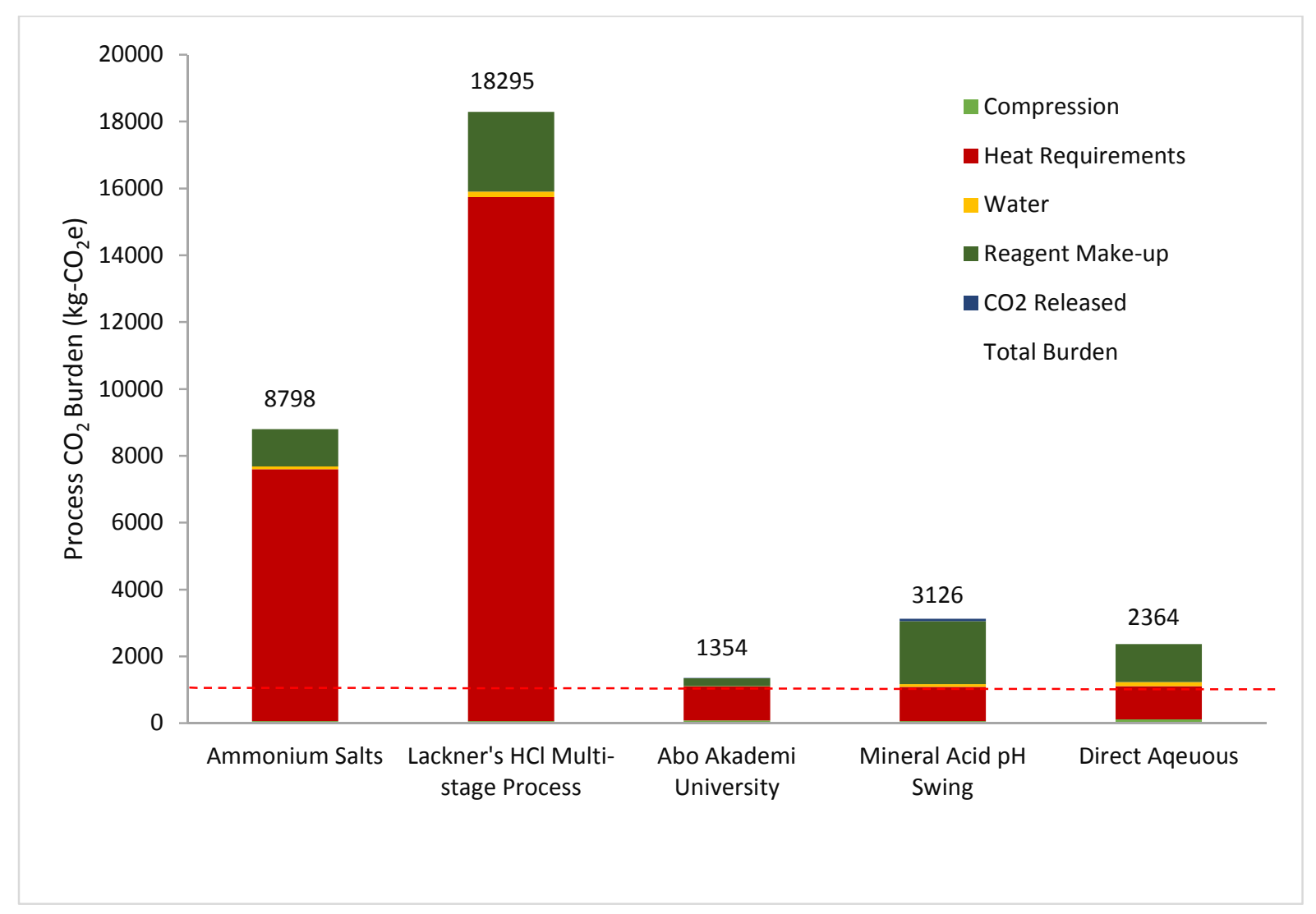

Figure 5-2: Comparison of carbon dioxide footprints of selected carbonation process system

The results in Figure 5-2 indicate that the mineral carbonation process having the highest carbon dioxide footprint is Lackner's multi-stage $\mathrm{HCl}$ process, followed by the ammonium salts process. This finding is consistent with the high heating requirements for these processes. The least emissions intensive process is the $\AA A U$ process, followed by the direct aqueous process and the mineral acid $\mathrm{pH}$ swing process. 
However, in terms of carbon neutrality, none of the process resulted in a net reduction in carbon dioxide emissions. This means that the amount of carbon dioxide emitted exceeds the amount sequestered, particularly in the cases of Lackner's multi-stage $\mathrm{HCl}$ process and the ammonium salts process. The processes were designed for the sequestration of $1000 \mathrm{~kg} / \mathrm{hr}$ of carbon dioxide, and the closest to achieving this target is the ÅU process, with net emissions of 354 $\mathrm{kg}-\mathrm{CO}_{2} \mathrm{e}$.

\subsubsection{Process Contributions}

Table 5-2 summarises the percentage contributions of the various processes to total carbon dioxide emissions.

Table 5-2: Percentage contributions of process requirements to total carbon dioxide emissions

\begin{tabular}{|l|r|r|r|r|r|}
\hline \multicolumn{1}{|c|}{ Description } & $\begin{array}{l}\text { Ammonium } \\
\text { Salts }\end{array}$ & $\begin{array}{l}\text { Lackner's } \\
\text { HCl Multi- } \\
\text { stage Process }\end{array}$ & $\begin{array}{l}\text { Abo Akademi } \\
\text { University }\end{array}$ & $\begin{array}{l}\text { Mineral } \\
\text { Acid pH } \\
\text { Swing }\end{array}$ & $\begin{array}{l}\text { Direct } \\
\text { Aqueous }\end{array}$ \\
\hline Compression & $1 \%$ & $0 \%$ & $6 \%$ & $2 \%$ & $5 \%$ \\
\hline Heat Requirements & $86 \%$ & $86 \%$ & $75 \%$ & $33 \%$ & $42 \%$ \\
\hline Water & $1 \%$ & $1 \%$ & $0 \%$ & $2 \%$ & $5 \%$ \\
\hline Reagent Make-up & $13 \%$ & $13 \%$ & $17 \%$ & $60 \%$ & $48 \%$ \\
\hline $\mathrm{CO}_{2}$ Released & $0 \%$ & $0 \%$ & $1 \%$ & $2 \%$ & $0 \%$ \\
\hline
\end{tabular}

The results in Table 5-2 demonstrate that heat requirements and chemical reasgent make-up are the most emissions intensive processes involved in the mineral carbonation systems investigated. This appears to suggest that heat and chemical reagent requirements are a good predictor of process performance from a carbon emissions perspective, at least for the processes investigated in this study. Other process contributing to a minor extent are water supply and carbon dioxide compression. The effect of these processes on the overall carbon dioxide footprint are discussed in more detail in the sub-sections below.

\section{Process Heat Requirements}

For three of the five processes considered heat requirements are by far the largest emissions impact, accounting for up to $86 \%$ for Lackner's multi-stage $\mathrm{HCl}$ and the ammonium salts process as shown in Table 5-2. This percentage corresponds to a mammoth $15677 \mathrm{~kg}-\mathrm{CO}_{2} \mathrm{e}$ in emissions. Even for the case where heat requirements are not the largest contributor (mineral acid $\mathrm{pH}$ swing and direct aqueous processes) they still account for a considerable share of the carbon footprint. This means that supply of heat requirements are an important consideration for the mineral carbonation process. This finding is consistent with observations made by a number of other authors and has been identified as one of the issues to resolved for mineral 
carbonation to be successful in several state of the art reviews (Huijgen and Comans, 2003; Olajire, 2013; Sanna et al., 2014b).

\section{Chemical Reagent Requirements}

As can be seen in Figure 5-2, the second most (when not the first) carbon intensive process element for most of the mineral carbonation systems is reagent make-up. The reagents vary across the selected mineral carbonation processes, and include inorganic acids, ammonium salts, and inorganic salts such as sodium bicarbonate. For most processes (ammonium salts, Lackner's $\mathrm{HCl}$ multi-stage, direct aqueous, mineral acid $\mathrm{pH}$ swing) reagent production alone resulted in carbon dioxide emissions that exceed the amount sequestered, that is, resulted in the process being carbon positive (Table 5-3).

Table 5-3: Carbon dioxide emissions for chemical reagent make-up

\begin{tabular}{|l|c|}
\hline \multicolumn{1}{|c|}{ Mineral Carbonation Process } & $\begin{array}{c}\text { Reagent Make-up Carbon Dioxide } \\
\text { Footprint (kg-CO2e) }\end{array}$ \\
\hline Ammonium Salts & 1113 \\
\hline Lackner's HCl Multi-stage Process & 2394 \\
\hline Abo Akademi University & 232 \\
\hline Mineral Acid pH Swing & 1881 \\
\hline Direct Aqueous & 2557 \\
\hline
\end{tabular}

Studies in the literature have largely ignored the contribution of reagent make-up, or have been based on the assumption that $100 \%$ reagent recovery can be achieved (Nduagu et al., 2012b; Dri, 2014). This is unlikely to be the case in practice due to inevitable material losses in the process, as well as the need to purge in order to remove accumulating components. The potential impact of reagent make-up on the carbon dioxide emissions attributed to the mineral carbonation process highlights the importance of taking these contributions into account.

\section{Water, Compression and $\mathrm{CO}_{2}$ Released}

Whilst the impact of water on process heat requirements was substantial (as discussed in Section 5.1), the carbon dioxide emissions associated with the supply of fresh water on carbon dioxide emissions is comparatively insignificant, particularly in the Åbo Akademi University process which uses relatively low quantities of feed water. In general, the emissions associated with the supply of water are less than $5 \%$ for all the selected mineral carbonation process systems (Table 5-2). The impact of compression of carbon dioxide on the total carbon dioxide footprint is also largely minimal, with the contribution not being more than $6 \%$ of the total emissions impact. As discussed earlier (Section 3.2.1), this included the compression for 
transport by pipeline as well as any additional compression required to raise the pressure from the pipeline pressure for process purposes.

Another source of emissions that comes with some of the selected processes is the release of unreacted carbon dioxide. This is a factor for the Åbo Akademi University process as well as the mineral acid $\mathrm{pH}$ swing process, with the amount of carbon dioxide released accounting for $1 \%$ and $2 \%$ of the total emissions, respectively.

\subsection{The Potential Viability of Selected Mineral Carbonation Processes}

This section discusses general factors influencing the potential viability from both a technical and carbon neutrality perspective.

\subsubsection{Process Technical Feasibility}

It has been noted that pyroxene is stable, and sparingly reactive in comparison to other silicate minerals such as serpentine and olivine (Meyer et al., 2014; Sanna et al., 2014a) in terms of the extraction of magnesium cations from the silicate mineral matrix. This is a limiting step in the mineral carbonation of PGM tailings. Though all of the processes considered have been shown to be carbon positive, it important to consider the technical viability of the processes in the event that progress is made in future studies to make them sustainable in terms of resulting in a net reduction of carbon dioxide emissions.

The ammonium salts process is an integrated process based on the use of ammonium-based reagents for extraction, capture and carbonation. The extraction of magnesium from pyroxene has been demonstrated by Sanna et al. (2014a). The maximum extraction efficiencies achieved were a modest $30 \%$ in comparison to more than $90 \%$ for serpentine. Despite the low extraction efficiencies, it is foreseeable that this process could be implementable from a technical standpoint should improvements be made to its carbon neutrality. Numerous chemical processes operate at low single pass conversions, for example the production of ammonia via the HaberBosch process, but through the use of recycles are not only technically feasible, but economically profitable too. This leads to an assessment that from a technical perspective, the ammonium salts process could be feasible for the extraction of magnesium from pyroxene-rich PGM tailings and subsequent carbonation.

It has been mentioned that the mineral carbonation process proposed by Lackner et al. (1995) was based on theory only (Section 4.2). However, the extraction of magnesium from PGM tailings using hydrochloric acid has been demonstrated by Vogeli (2012). The author reported magnesium extraction efficiencies of up to $20 \%$ for the various samples considered. Through 
a similar rationale made for the ammonium salts process, the process could be considered technically feasible and viable should improvements be implemented that move it towards carbon neutrality.

The Åbo Akademi University process is a gas-solid mineral carbonation that includes regeneration of chemical reagents through precipitation and evaporation. This process, though carbon positive, is one of the more promising from a carbon dioxide emissions point of view. However, the experiments conducted at the Åbo Akademi University have been focused on serpentine as the silicate mineral feedstock. Studies using pyroxene-based feedstock have not been conducted. Considering that the extraction efficiencies of the more reactive serpentine have been around $60-70 \%$, without experimental work it is impossible to predict the extent, and even the possibility of reactivity of pyroxene-rich PGM tailings. Thus the technical feasibility of implementing this process is subject to experimental studies conducted to evaluate the possibility of extraction of magnesium from PGM tailings using this approach.

The mineral acid $\mathrm{pH}$ swing process has been developed from isolated experimental results obtained by Meyer (2014) and Vogeli (2012). It has not been developed into a complete carbonation process, and the chemistry has not been well defined. The authors reported an extraction efficiency of up to $20 \%$ using hydrochloric acid on PGM tailings. As mentioned earlier, with the use of recycle streams the overall conversion can be significantly improved in comparison to the relatively modest single pass conversion. However, the assumption that it is possible to recover and regenerate hydrochloric acid during the carbonation stage has not been validated experimentally, particularly at the low temperatures associated with the carbonation step. The technical feasibility of this process hinges on the feasibility of low temperature regeneration of the acid reagent, which has typically been achieved at higher temperatures (Lackner et al., 1995; Teir, 2008).

The direct aqueous process proposed and developed at the National Energy Technology Laboratory is a water-based extraction and carbonation single-step process. This process has been developed on the basis of experimental data for studies conducted on serpentine, olivine and wollastonite by the reseachers (O'Connor et al., 2005). The authors reported good conversions of more than $80 \%$ in some cases. Extraction and carbonation experiments using pyroxene or pyroxene-rich feedstock were not conducted by the authors. It is expected that pyroxene-rich PGM tailings, due to their stable nature, will be sparingly reactive under these conditions as Koukouzas et al. (2009) reported poor carbonation of harzburgite and pyroxenite 
using this approach, under similar conditions. Additionally, under the mildly acidic conditions used by Meyer et al. (2014) the reported extraction efficiencies of magnesium were less than $5 \%$, albeit at more moderate conditions compared to those used in the direct aqueous carbonation process. These low single pass conversions would require prohibitively large recycle loops to be implemented. Further empirical studies would thus need to be conducted in order to establish whether higher conversions can be achieved.

\subsubsection{Heat Integration Potential}

Heat integration has the potential to reduce external process heat demands, through the heat exchange of available heat between process streams. However, even with heat integration, not all available heat in process streams can be recovered due to thermodynamic constraints, as well as safety and process control considerations (Papoulias and Grossmann, 1983). The technique used for heat integration and heat exchanger network design is primarily pinch analysis. This has been reported to typically achieve energy savings of 20-30\% (Linnhoff and Hindmarsh, 1983), although higher savings have been reported in some chemical processes (Kemp, 2007). Although limited studies have been conducted to rigorously evaluate the potential for heat integration in mineral carbonation processes, the benefit of heat integration was demonstrated through the work conducted by Nduagu et al. (2012b). These researchers were able to achieve a $35 \%$ reduction in the carbon dioxide footprint through heat integration, alongside systems expansion and mass allocation. A number of researchers have also suggested the use of reaction and exit stream heat for the pre-heating of the inlet stream in the direct aqueous process developed at the NETL (O'Connor et al., 2005; Nduagu et al., 2012b; Giannoulakis et al., 2014).

It should, however, be noted that in the case of Lackner's multi-stage $\mathrm{HCl}$ and ammonium salts processes heating requirements have to be reduced by more than $90 \%$. This suggests that even with heat integration, the benefits in terms of making these processes sustainable from a carbon dioxide balance perspective are unlikely to be sufficient. On the other hand, the heat requirements for the direct aqueous process could be reduced through the use of the reactor exit stream to pre-heat the aqueous reagent feed stream, which is a major heat consumer, resulting in a potential reduction in external heat of about $70 \%$. The reduction of the contribution to the footprint of heat requirements in the AAU process is also potentially feasible. The footprint associated with heat requirements for this process is just over $1000 \mathrm{~kg}$ $\mathrm{CO}_{2} \mathrm{e}$ which, with a $30-50 \%$ reduction, could render this process a net carbon sink. 


\subsubsection{Additional Process Impacts and Plant Construction}

The study did not include the energy consumption associated with pumping, separations and mixing, as it was assumed that this would to be much less than that associated with typically intensive unit operations such as heating and compression. Additionally, the impact of materials of construction, as well as energy associated with the actual construction of the mineral carbonation plant have not been considered. Inclusion of these factors can be expected to increase both the energy consumption and the total carbon dioxide emissions for the mineral carbonation process systems. The quantification of these emissions would need to be included in more detailed analyses of promising processes.

\subsubsection{Chemical Reagent Recovery, Recycles and Losses}

The recovery of chemical reagents, through the implementation of recycles streams in the flowsheet results in significantly less reagent make-up. However, due to the presence of some reaction products (such as silicon dioxide, that is recycled alongside reagent pyroxene), and excessive water in the recycle stream, that accumulate with recirculation, as well as requirements for excess feed into reactors, some reagent is lost as part of purges that remove these components, in order to avoid a build-up of these components, or excessively large recycle streams. The size of these purge streams varied generally between $10-15 \%$ of the splitter input stream, and was assumed to account for these losses, as well as losses associated with spent reagent and typical plant losses through for example leaks and evaporation. This loss is accounted for by the make-up reagent. In turn, the make-up reagent incurs a carbon footprint associated with its production. In this regard, there is an incentive to minimise the make-up reagent in order to reduce its impact on the carbon footprint. Reagent make-up accounts for a significant fraction of the carbon footprint, particularly in the case of the direct aqueous and mineral acid $\mathrm{pH}$ swing process $(48 \%$ and $60 \%$ of the total carbon footprint, respectively). Furthermore, in four of the five selected processes (Lackner's multi-stage $\mathrm{HCl}$, direct aqueous, ammonium salts, mineral acid $\mathrm{pH}$ swing) the carbon dioxide footprint contributions from the make-up of reagent exceeds the carbon dioxide sequestered by the process. It can be argued that the size of the recycle streams for the processes could be increased by adjusting the recycle ratio to reduce dependence on external reagent supply. However, increasing the recycle ratio may affect plant sizing, increase heat requirements, and also impact process stability as discussed in Chapter 4. In this regard, exploring optimal recycle ratios that balance these trade-offs to minimize the carbon footprint could be explored. 


\section{Chapter 6}

\section{Conclusions and Recommendations}

\subsection{Background and Motivation}

Mineral carbonation has been identified as a potential carbon dioxide emissions reduction strategy. This process uses minerals, rich in magnesium or calcium, to permanently store gaseous carbon dioxide emissions in metal carbonate form. Several mineral carbonation process routes have been proposed ranging from direct gas-solid to indirect aqueous processes. The mineral carbonation process, though thermodynamically favourable, has been noted to be slow. As a result, a large body of work has been conducted to accelerate the carbonation process so that it may be justified for industrial implementation. These studies have been primarily focused on the more reactive and abundant silicate minerals such as serpentine and olivine. Consequently, proposed mineral carbonation processes have been developed on the basis of these minerals as feedstock. The acceleration of the carbonation process has also involved the use of chemical reagents, high temperatures and high pressures to enhance reaction kinetics. This has the potential to significantly increase the material and energy requirements of processes, and thus the carbon footprint. With limited comprehensive studies to evaluate the environmental implications of mineral carbonation, from a carbon neutrality perspective, a knowledge gap exists, particularly with regards to less reactive silicate mineral feedstock such as pyroxene-rich PGM tailings.

\subsection{Research Objectives}

South Africa has a large PGM industry that produces huge quantities of tailings that have been identified to have a potential for $\mathrm{CO}_{2}$ sequestration. The over-arching objective of the study was to investigate the viability of using PGM tailings to sequester carbon dioxide on the basis of carbon neutrality. This study set out to achieve this by defining the following aims:

I. Identify potentially feasible flowsheets for the mineral carbonation of PGM tailings on the basis of literature.

II. Develop mass and energy balances for the identified processes using process simulation software. 
III. Establish the carbon footprint of the process using Life Cycle Assessment (LCA) software.

\subsection{Key Research Findings}

This section summarises the key findings of the study presented in Chapters 2, 4 and 5.

\subsubsection{Identification of Potentially Feasible Mineral Carbonation Processes}

The first component of this study involved the identification of potentially feasible processes for the mineral carbonation of pyroxene-rich PGM tailings feedstock. This was achieved by conducting a detailed review and assessment of the available literature, in order to establish existing technologies and techniques that have been developed for mineral carbonation. The literature review demonstrated that several process routes exist for the mineral carbonation of silicate minerals, and that within these routes a considerable amount of research that has been done to understand and improve the performance of the processes. A general trend towards multi-stage processes such as in the gas-solid and $\mathrm{pH}$ swing processes was observed, whilst some researchers have continued developing single step process such as the direct aqueous process. The focus on accelerating process kinetics through temperature and pressure increases, as well as chemical reagent use by researchers was noted, as well as the development of processes with a focus on reagent regeneration. This was in contrast to the limited and underdeveloped studies that evaluate the effectiveness of the processes from a carbon balance or lifecycle assessment (LCA) perspective. Additionally, few of the processes have been developed beyond the laboratory scale. The brine solutions based SkyMine process is the only known commercial application to date.

On the basis of this survey, five processes were selected for further study: One multi-stage gassolid process and four aqueous-based carbonation processes. These were:

1. Ammonium Salts Process - Indirect Aqueous pH Swing

2. Lackner's Multi-stage $\mathrm{HCl}$ Process - Indirect Aqueous Multi-stage

3. Åbo Akademi University ( $\AA \mathrm{AU})$ Process - Indirect Gas-Solid Multi-stage

4. Mineral Acid pH Swing Process - Indirect Aqueous pH Swing

5. Direct Aqueous Process - Direct Aqueous

\subsubsection{Material and Energy Balance Simulations}

The simulations of these processes were then developed, through the use of AspenPlus simulation software v8.0, for the purpose of establishing the flows of materials and energy within the selected mineral carbonation process systems. These were based on the sequestration 
of 1 ton of carbon dioxide by pyroxene (as a proxy for PGM tailings). This served as a basis for carbon dioxide emissions accounting. The external material requirements of selected processes are vary since these processes make use of different chemical reagents, and operate at different reaction efficiencies.

Heat requirements were noted to account for the majority of energy requirements, hence were selected as the focus for further analysis. The process that required the most heat was Lackner's multi-stage process $\left(230 \times 10^{3} \mathrm{MJ} /\right.$ ton- $\mathrm{CO}_{2}$ sequestered $)$, whereas the direct aqueous process required the least $\left(6 \times 10^{3} \mathrm{MJ} /\right.$ ton- $\mathrm{CO}_{2}$ sequestered). An analysis of heat requirements showed that aqueous processes generally required much more energy than the gas-solid ÅU process, with the exception of the direct aqueous process. This was linked to the quantity of water used in the aqueous processes, which resulted in substantially higher energy requirements. This highlighted a short coming in the aqueous processes, that the benefits of process acceleration in aqueous media result in significant increases in heat requirements.

\subsubsection{Mineral Carbonation Process Sustainability - Effectiveness to Sequester $\mathrm{CO}_{2}$}

The fundamental proposal of mineral carbonation is to be an effective carbon sink, that is, to reduce the overall carbon dioxide emissions released into the atmosphere. Consequently, the carbon dioxide balance is a good measure of the viability of a particular carbonation process. In this regard, the global warming potential $\left(\mathrm{kg}-\mathrm{CO}_{2} \mathrm{e}\right)$ of the process, which is a measure used to define greenhouse gas emissions in terms of a common unit related to the heat the greenhouse gas traps relative to carbon dioxide, was compared against the amount of carbon dioxide the process sequesters. The accounting of carbon dioxide emissions was conducted using SimaPro v7.7.3. background process modelling software. The carbon dioxide footprints for all selected processes were calculated and the carbon dioxide balance established. It was found that all selected processes were carbon positive, that is, ineffective as net carbon sinks.

The largest contributors to the overall emissions were primarily heat requirements and chemical reagent make-up. The two contributions were so large that they generally accounted for more than $85 \%$ of the total emissions of the selected processes, when combined. In the cases of the ammonium salts and Lackner's Multi-stage $\mathrm{HCl}$ processes this amounted to $98 \%$ and $99 \%$, respectively. These were thus identified as key areas to focus efforts to reduce the overall emissions impact of mineral carbonation processes. 
Lackner's multi-stage $\mathrm{HCl}$ process was found to be the have the highest carbon footprint, resulting in 18.2 tons of carbon dioxide emissions to sequester 1 ton of carbon dioxide. The second largest emitter was the ammonium salts process emitting eight times more $\mathrm{CO}_{2}$ than it is intended to sequester. The mineral acid $\mathrm{pH}$ swing process resulted in three times more emissions than sequestered. The process with the least carbon dioxide footprint was the Åbo Akademi University ( $\AA \mathrm{AU})$ process, with just $354 \mathrm{~kg}-\mathrm{CO}_{2} \mathrm{e}$ of additional emissions. The direct aqueous process resulted in about $2400 \mathrm{~kg}-\mathrm{CO}_{2} \mathrm{e}$ in emissions.

Generally, the aqueous mineral carbonation processes were considerably more emissions intensive than the gas-solid multi-stage $\AA A U$ process. This was linked to the higher quantity of water used by the aqueous processes in comparison to the $\AA A U$ process. It was observed that the large quantities of water used by these processes and changes of phase through evaporation substantially increase the energy requirements and thus the carbon dioxide footprint.

\subsection{Recommendations}

Although the selected processes were demonstrated to result in more carbon dioxide emissions than they sequester, the development of effective processes for the sequestration of carbon dioxide remains of key importance in terms of managing climate change. It is paramount that work in this strategic area is continued. However, it is evident that in some cases a lot of work still needs to be done to ensure that implemented mineral carbonation processes are reducing overall carbon dioxide emissions. In the case of mineral carbonation, it may be possible to reduce the carbon intensity of some of these processes sufficiently to render them net carbon negative. This is particularly the case for the three processes with the lowest carbon footprint: the mineral acid pH swing, the direct aqueous process, and more especially the Åbo Akademi University ( $\AA \mathrm{AU})$ process. It should, however, be noted that apart from limited preliminary experimental work on the mineral acid $\mathrm{pH}$ swing process, the technical feasibility of using pyroxene (the major component of PGM tailings) as feedstock for the processes has not been established to date. Preliminary testwork is thus recommended in the first instance to investigate the technical feasibility of using pyroxene as a feedstock for the direct aqueous and the $\AA \mathrm{AU}$ mineral carbonation processes.

In the second instance, further work needs to be conducted to reduce the carbon footprint on processes that are potentially feasible. This pertains in particular to the reduction of carbon 
emissions associated with heating and the production of chemical reagents as feed to the process.

\subsubsection{Reducing heating related carbon emissions}

Process heat requirements have been identified as one of the two largest contributors to the emissions footprint of mineral carbonation processes. The two components of the emissions impact related to process heat requirements are the source of the heat, and the quantity of heat required. In this regard, the options for reducing emissions related to heat generation would be to either use an alternative heat source or to reduce the quantity of heat required, though the former would benefit all process cases.

\section{The use of cleaner fuel sources for heat supply}

The current source used to generate heat for the selected mineral carbonation processes is natural gas. This is considered the cleanest fossil fuel, and also the cheapest. However, it is still a fossil fuel and thus releases considerable amounts of carbon dioxide when burned. An alternative to consider may be the use of biomass as a source, which has been suggested to achieve near zero net emissions since the source (plants and other organic matter) absorbs carbon dioxide during its lifetime (European Climate Foundation, 2010). The European Climate Foundation (2010) suggests a reduction in carbon dioxide emissions of up to $98 \%$ by using biomass instead of fossil fuels. However, biomass has a low energy density at $8 \mathrm{GJ} / \mathrm{t}$ in comparison to natural gas at $56 \mathrm{GJ} / \mathrm{t}$ (McKendry, 2002) and is more expensive per MWh (European Climate Foundation, 2010).

\section{The reduction of external heat supply}

The reduction of the overall quantity of heat required is an equally, if not more, important lever in the reduction of emissions associated with heat generation. This can be accomplished through exploring heat integration opportunities, and using low temperature process operations.

Heat integration involves the recovery or reuse of process heat by matching process streams that require heat (cold streams) to those that carry heat (hot streams). This minimizes the external utility requirements of the process, and thus the emissions impact associated with the generation of utilities. The design of the heat exchanger system is typically conducted using techniques like Pinch Technology to match cold and hot streams effectively. The mineral carbonation processes could benefit from the exploration of heat integration and effective heat 
exchanger network design. Hence, it is recommended that this be included in future work, so that the extent to which it can result to energy savings can be established.

The use of high temperatures to regenerate reagent, particularly in aqueous processes, results in a considerable footprint, from a carbon dioxide emissions perspective. Although processes that have integrated chemical reagent regeneration capabilities are attractive from a material use standpoint, it has been shown in this study that they can also incur huge energy penalties, primarily associated with regeneration. This highlights the importance of developing low temperature regeneration processes. Ideally, this would not just be limited to regeneration, but be the case for the entire process. The idea of using low temperature options has also been proposed by Sanna et al. (2014b), although few practical examples exist, particularly for reagents like ammonium bisulphate and hydrochloric acid. This suggests that additional work should be done in the mineral carbonation field, in general, to develop regeneration operations for promising processes that use more moderate temperatures. Otherwise, a re-evaluation of the balance between the costs and benefits of regeneration will have to be considered.

\subsubsection{Reducing material and chemical related carbon emissions}

The mineral carbonation process in its simplest form would be a direct gas-solid reaction between the silicate mineral and carbon dioxide. However, the kinetics of this reaction are too slow to be justifiable for industrial operation. This necessitates the use of chemical reagents to accelerate the carbonation process. This typically involves the use of acids, such as hydrochloric acid and ammonium salts for extraction, as well as bases such as ammonium and sodium hydroxide, and salts such as sodium chloride and sodium bicarbonate.

The use of chemical reagents has been identified as one of the major contributors in terms of carbon footprint for selected mineral carbonation processes. This emissions contribution is a function of the reagent production processes as well as the required quantities of reagent. This implies that these are the two levers of control for carbon dioxide emissions associated with chemical reagent use in the carbonation process.

\section{The use of cleaner input materials}

In most cases, the production of chemical reagents used in the mineral carbonation process involves energy intensive operations. For example, the production of hydrochloric acid using the direct synthesis approach is an energy intensive operation since a $2000{ }^{\circ} \mathrm{C}$ burner is used to facilitate the burning of chlorine in hydrogen (Austin and Glowacki, 2005). Considering other less energy intensive production processes such as the Mannheim process, or sourcing it 
as a by-product of other chemical processes such as the chlorination process could potentially reduce this emissions contribution. In this regard, using chemical reagent that are produced by cleaner processes in terms of carbon emissions is important for the reduction of this contribution. Additionally, exploring alternative reagents to achieve the same function in the process could also be useful in reducing the emissions contribution of chemical reagent makeup.

\section{The reduction of make-up reagent consumption}

The quantities of chemical reagent used in the process also affects the carbon emissions impact associated with external reagent production. The chemical reagents required vary from about $2.0 \mathrm{~kg} / \mathrm{kg}-\mathrm{CO}_{2}$ for Lackner's multi-stage $\mathrm{HCl}$ process to as low as $0.4 \mathrm{~kg} / \mathrm{kg}-\mathrm{CO}_{2}$ for the $\AA \mathrm{AU}$ process. A reduction in the amount of make-up reagent could be effected by either increasing recycle ratios or improving reaction efficiencies by altering process conditions. However, it must be noted this may affect process performance from a technical perspective, and also from an energy point of view.

\subsection{Concluding Remarks}

This thesis has highlighted the importance of adopting a holistic life cycle based approach to the evaluation of the performance of mineral carbonation processes. It has shown that enhancing process kinetics in isolation could result in processes that, whilst technically justifiable, may result in a net release of carbon dioxide emissions, further exacerbating the problem of global warming. However, mineral carbonation is an important carbon dioxide emissions reduction technology that should not be abandoned, considering the concerns with geological and ocean storage. It promises a permanent and safe option for the storage of carbon dioxide emissions, which could also make use of mineral industry waste streams. This has the potential to both reduce environmental impacts and enhance natural resource efficiency, through the improved utilisation of mined mineral material. However, it is clear that the technology is far from being a fully developed carbon dioxide emissions mitigation strategy. In particular, this study has identified a reduction in emissions associated with heating and production of chemical reagents as two key areas requiring attention. These emissions will need to be reduced substantially in most of the processes investigated, including the Lackner's multi-stage, ammonium salts, direct aqueous, and mineral acid $\mathrm{pH}$ swing processes. Developing low-temperature process operations, particularly for reagent regeneration, and optimising materials and energy use, whilst maintaining process kinetics performance, is the key to unlocking the potential of this technology. This is particularly important in the context 
of PGM tailings, which is a less reactive feedstock, but offers the potential for recovery of additional PGM mineral value, which would improve the economics of potential processes. This will require not just a modification of existing processes, but also a shift in the underlying process design philosophy towards a holistic approach, that takes into account kinetic performance and environmental performance, with the next frontier being the improvement of the economics of the technology. 


\section{References}

Alexander, G., Mercedes Maroto-Valer, M. \& Gafarova-Aksoy, P. 2007. Evaluation of reaction variables in the dissolution of serpentine for mineral carbonation. Fuel. 86(1):273-281.

Anglo American Platinum 2013. Anglo American Platinum Annual Report. South Africa: Anglo American Platinum.

Appl, M.,. 2007. "Ammonia", Ullmann's Encyclopedia of Industrial Chemistry. 7th ed. Germany: Wiley-VCH.

Aspen Plus. 2012. Getting Started Building and Running a Process Model. v8. AspenTech.

AspenTech. 2013. Aspen Physical Property System - Physical Property Methods. AspenTech.

Austin, S., \& Glowacki, A.,. 2005. "Hydrochloric Acid", Ullmann's Encyclopedia of Industrial Chemistry. 7th ed. Germany: Wiley-VCH.

Azdarpour, A., Asadullah, M., Mohammadian, E., Hamidi, H., Junin, R. \& Karaei, M.A. 2015. A review on carbon dioxide mineral carbonation through $\mathrm{pH}$-swing process. Chemical Engineering Journal. 279:615-630. DOI:http://dx.doi.org/10.1016/j.cej.2015.05.064.

Baukal, C.E.,. 2000. Heat Transfer in Industrial Combustion. United States: CRC Press.

Bodénan, F., Bourgeois, F., Petiot, C., Augé, T., Bonfils, B., Julcour-Lebigue, C., Guyot, F., Boukary, A. et al. 2014. Ex situ mineral carbonation for $\mathrm{CO}_{2}$ mitigation: Evaluation of mining waste resources, aqueous carbonation processability and life cycle assessment (Carmex project). Minerals Engineering. 59:52-63.

Bonfils, B., Julcour-Lebigue, C., Guyot, F., Bodénan, F., Chiquet, P. \& Bourgeois, F. 2012. Comprehensive analysis of direct aqueous mineral carbonation using dissolution enhancing organic additives. International Journal of Greenhouse Gas Control. 9:334346.

Brantley, S.L. 2008. Kinetics of mineral dissolution. In Kinetics of water-rock interaction. Springer. 151-210.

British Petroleum 2013. BP Statistical Review of World Energy. United Kingdom: British Petroleum.

Butt, D., Lackner, K., Wendt, C., Conzone, S., Kung, H., Lu, Y., \& Bremser, J., 1996. Kinetics of Thermal Dehydroxylation and Carbonation of Magnesium Hydroxide. Journal of the American Ceramic Society. 79(7):1892-1898.

Cloete, M., 2006. Draft Framework for Geological and Mineralogical Storage of $\mathrm{CO}_{2}$ in South Africa. South Africa: Council for Geoscience. 
Cloete, M., 2010. Atlas on Geological Storage of Carbon Dioxide in South Africa. South Africa: Council for Geoscience.

Department of Environmental Affairs 2011. National Climate Change Response White Paper. South Africa: Department of Environmental Affairs.

Doucet, F., 2011. Scoping Study on $\mathrm{CO}_{2}$ Mineralization Technologies. Pretoria: Council for Geoscience.

Dri, M., 2014. Utilization of industrial waste for value-added permanent sequestration of $\mathrm{CO}_{2}$. PhD. Heriot-Watt University.

Dri, M., Sanna, A., \& Maroto-Valer, M., 2014. Mass and energy balance of $\mathrm{NH}_{4}$-salts $\mathrm{pH}$ swing mineral carbonation process using steel slag. Energy Procedia. 63:6544-6547.

Eloneva, S., Teir, S., Savolahti, J., Fogelholm, C., \& Zevenhoven, R., 2007. Co-utilization of $\mathrm{CO}_{2}$ and calcium silicate-rich slags for precipitated calcium carbonate production (part II). Proceedings of ECOS 2007. 25-28 June. Italy: 1389-1396.

Environmental Information Administration 2014. EIA Energy - South Africa. United States of America: Environmental Information Administration.

Environmental Protection Agency (EPA) 2006. Life Cycle Assessment: Principles and Practice. (EPA/600/R-06/060). United States: Environmental Protection Agency.

European Climate Foundation 2010. Biomass for heat and power-opportunity and economics. European Climate Foundation.

Fabian, M., Shopska, M., Paneva, D., Kadinov, G., Kostova, N., Turianicová, E., Briančin, J., Mitov, I. et al. 2010. The influence of attrition milling on carbon dioxide sequestration on magnesium-iron silicate. Minerals Engineering. 23(8):616-620.

Fagerlund, J., Nduagu, E., Romão, I. \& Zevenhoven, R. 2010. A stepwise process for carbon dioxide sequestration using magnesium silicates. Frontiers of Chemical Engineering in China. 4(2):133-141.

Fagerlund, J., Teir, S., Nduagu, E. \& Zevenhoven, R. 2009. Carbonation of magnesium silicate mineral using a pressurised gas/solid process. Energy Procedia. 1(1):4907-4914.

Finnveden, G., Hauschild, M., Ekvall, T., Guinee, J., Heijungs, R., Hellweg, S., Koehler, A., Pennington, D., et al. 2009. Recent developments in Life Cycle Assessment. Journal of Environmental Management. 91:1-21.

Forbes, S.M., Verma, P., Curry, T.E., Friedmann, J.S., \& Wade, S.M., 2008. Guidelines for Carbon Dioxide Capture, Transport, and Storage. United States: World Resources Institute (WRI). 
Gerdemann, S., Dahlin, D., O'Connor, W., \& Penner, L., 2002. Carbon Dioxide Sequestration by Aqueous Mineral Carbonation of Magnesium Silicate Minerals. United States: Albany Research Center.

Gerdemann, S.J., O'Connor, W.K., Dahlin, D.C., Penner, L.R. \& Rush, H. 2007. Ex situ aqueous mineral carbonation. Environmental Science \& Technology. 41(7):2587-2593.

Ghoorah, M., Dlugogorski, B.Z., Oskierski, H.C. \& Kennedy, E.M. 2014. Study of thermally conditioned and weak acid-treated serpentinites for mineralisation of carbon dioxide. Minerals Engineering. 59:17-30.

Ghoorah, M., Dlugogorski, B.Z., Oskierski, H.C. \& Kennedy, E.M. 2014. Study of thermally conditioned and weak acid-treated serpentinites for mineralisation of carbon dioxide. Minerals Engineering. 59:17-30.

Giannoulakis, S., Volkart, K., \& Bauer, C., 2014. Life cycle and cost assessment of mineral carbonation for carbon capture and storage in European power generation. International Journal of Greenhouse Gas Control. 21:140-157.

Global Carbon Project 2015. The top 20 carbon dioxide emitting countries. Available: http://www.globalcarbonatlas.org/?q=en/emissions [19 June 2015].

Goedkoop, M., Heijungs, R., Huijbregts, M., Schryver, A., Struijs, J., \& van Zelm, R., 2013. ReCiPe 2008 - A life cycle impact assessment method which comprises harmonised category indicators at the midpoint and the endpoint level. (I). Netherlands: .

Guinée, J., Gorree, M., Heijungs, R., Huppes, G., Kleijn, R., de Koning, A., van Oers, L., Sleeswijk, W., et al. 2002. Handbook on Life Cycle Assessment: Operational Guide to the ISO Standards. Dordrecht: Kluwer Academic Publishers.

Hänchen, M., Prigiobbe, V., Storti, G., Seward, T. \& Mazzotti, M. 2006. Dissolution kinetics of fosteritic olivine at $90-150^{\circ} \mathrm{C}$ including effects of the presence of $\mathrm{CO}_{2}$. Geochimica Et Cosmochimica Acta. 70(17):4403-4416.

Haug, T., Munz, I. \& Kleiv, R. 2011. Importance of dissolution and precipitation kinetics for mineral carbonation. Energy Procedia. 4:5029-5036.

Herrmann, I.T. \& Moltesen, A. 2015. Does it matter which Life Cycle Assessment (LCA) tool you choose? - a comparative assessment of SimaPro and GaBi. Journal of Cleaner Production. 86:163-169. DOI:http://dx.doi.org/10.1016/j.jclepro.2014.08.004.

Huijgen, W.J. \& Comans, R. 2007. Carbon dioxide sequestration by mineral carbonation. Wageningen Universiteit.

Huijgen, W.J.J. \& Comans, R.N.J. 2003. Carbon dioxide sequestration by mineral carbonation: Literature review. Energy research Centre of the Netherlands ECN. 
Huisingh, D., Zhang, Z., Moore, J., Qiao, Q., \& Li, Q., 2015. Recent advances in carbon emissions reduction: policies, technologies, monitoring, assessment and modeling. Journal of Cleaner Production. 103:1-12.

IEA Statistics 2013. $\mathrm{CO}_{2}$ emissions from fossil fuel combustion: Highlights. France: IEA Statistics.

Intergovernmental Panel on Climate Change 2005. Carbon Capture and Storage. United Kingdom: Cambridge University Press.

Intergovernmental Panel on Climate Change 2013. Climate Change 2013: The Physical Science Basis. Cambridge, United Kingdom: Cambridge University Press.

IPCC 2007. Climate Change 2007: The Physical Science Basis. United Kingdom: Intergovernmental Panel on Climate Change.

ISO. 2006. Environmental Management - Life Cycle Assessment - Principles and Framework. Switzerland: International Organization for Standardization.

Kakizawa, M., Yamasaki, A., \& Yanagisawa, Y., 2001. A new $\mathrm{CO}_{2}$ disposal process via artificial weathering of calcium silicate accelerated by acetic acid. Energy. 26:341-354.

Kelly, K., Silcox, G., Sarofim, A., \& Pershing, D., 2011. An evaluation of ex situ, industrial scale, aqueous $\mathrm{CO}_{2}$ mineralization. International Journal of Greenhouse Gas Control. 5(6):1587-1595.

Kemp, I.,. 2007. Pinch Analysis and Process Integration. 2nd ed. United Kingdom: Elsevier.

Khoo, H., \& Tan, R., 2006. Life Cycle Evaluation of $\mathrm{CO}_{2}$ recovery and Mineral Sequestration Alternatives. Environmental Progress. 25(3):208-217.

Kirchofer, A., Brandt, A., Krevor, S., Prigiobbe, V., \& Wilcox, J., 2012. Impact of alkalinity sources on the life-cycle energy efficiency of mineral carbonation technologies. Energy \& Environmental Science. 5:8631.

Kirk-Othmer. 2007. Kirk-Othmer Encyclopedia of Chemical Technology . 5th ed. United States: John Wiley \& Sons.

Klein, C., \& Dutrow, B.,. 2007. Manual of Mineral Science. 23rd ed. United States: Wiley.

Koornneef, J., Spruijt, M., Molag, M., Ramirez, A., Turkenburg, W., \& Faaij, A., 2010. Quantitative risk assessment of $\mathrm{CO}_{2}$ transport by pipelines - A review of uncertainties and their impacts. Journal of Hazardous Materials. 177:12-27.

Koukouzas, N., Gemeni, V. \& Ziock, H. 2009. Sequestration of $\mathrm{CO}_{2}$ in magnesium silicates, in Western Macedonia, Greece. International Journal of Mineral Processing. 93(2):179186. 
Krevor, S.C. \& Lackner, K.S. 2009. Enhancing process kinetics for mineral carbon sequestration. Energy Procedia. 1(1):4867-4871.

Kurt, C., \& Bittner, J.,. 2005. "Sodium Hydroxide", Ullmann's Encyclopedia of Industrial Chemistry. 7th ed. Germany: Wiley-VCH.

Lackner, K.S., Butt, D.P. \& Wendt, C.H. 1997. Progress on binding $\mathrm{CO}_{2}$ in mineral substrates. Energy Conversion and Management. 38, Supplement:S259-S264. DOI:http://dx.doi.org/10.1016/S0196-8904(96)00279-8.

Lackner, K.S., Wendt, C.H., Butt, D.P., Joyce Jr., E.L. \& Sharp, D.H. 1995. Carbon dioxide disposal in carbonate minerals. Energy. 20(11):1153-1170. DOI:http://dx.doi.org/10.1016/0360-5442(95)00071-N.

Leung, D.Y.C., Caramanna, G., \& Maroto-Valer, M.M. 2014. An Overview of current status of carbon dioxide capture and storage technologies. Renewable and Sustainable Energy Reviews. 39:426-443.

Li, W., Li, W., Li, B. \& Bai, Z. 2009. Electrolysis and heat pretreatment methods to promote $\mathrm{CO}_{2}$ sequestration by mineral carbonation. Chemical Engineering Research and Design. 87(2):210-215.

Liang, F., Ryvak, M., Sayeed, S., \& Zhao, N., 2012. The role of natural gas as a primary fuel in the near future, including comparisons of acquisition, transmission and waste handling costs of as with competitive alternatives. Chemistry Central Journal. 6:1-24.

Linnhoff, B. \& Hindmarsh, E. 1983. The pinch design method for heat exchanger networks. Chemical Engineering Science. 38(5):745-763. DOI:http://dx.doi.org/10.1016/00092509(83)80185-7.

Maroto-Valer, M., Fauth, D., Kuchta, M., Zhang, Y. \& Andresen, J. 2005. Activation of magnesium rich minerals as carbonation feedstock materials for $\mathrm{CO}_{2}$ sequestration. Fuel Processing Technology. 86(14):1627-1645.

McKendry, P. 2002. Energy production from biomass (part 2): conversion technologies. Bioresource Technology. 83(1):47-54. DOI:http://dx.doi.org/10.1016/S09608524(01)00119-5.

Meyer, N., Vögeli, J., Becker, M., Broadhurst, J., Reid, D. \& Franzidis, J. 2014. Mineral carbonation of PGM mine tailings for $\mathrm{CO}_{2}$ storage in South Africa: A case study. Minerals Engineering.

Meyer, N. 2014. An investigation into the dissolution of pyroxene: A precursor to mineral carbonation of PGM tailings in South Africa. Masters. University of Cape Town.

Moazzem, S., Rasul, M. \& Khan, M.M.K. 2013. Energy recovery opportunities from mineral carbonation process in coal fired power plant. Applied Thermal Engineering. 51(1):281291. 
Nduagu, E., Bergerson, J., \& Zevenhoven, R., 2012b. Life cycle assessment of $\mathrm{CO}_{2}$ sequestration in magnesium silicate rock - A comparative study. Energy Conversion and Management. 55:116-126.

Nduagu, E., Björklöf, T., Fagerlund, J., Wärn, J., Geerlings, H., \& Zevenhoven, R., 2012a. Production of magnesium hydroxide from magnesium silicate for the purpose of $\mathrm{CO}_{2}$ mineralization - Part 2: $\mathrm{Mg}$ extraction modeling and application to different $\mathrm{Mg}$ silicate rocks. Minerals Engineering. 30:87-94.

Nduagu, E., Fagerlund, J. \& Zevenhoven, R. 2012. Contribution of iron to the energetics of $\mathrm{CO}_{2}$ sequestration in $\mathrm{Mg}$-silicates-based rock. Energy Conversion and Management. 55:178-186. DOI:http://dx.doi.org/10.1016/j.enconman.2011.10.023.

Newall, P., Clarke, S., Haywood, H., Scholes, H., Clarke, N., King, P., \& Barkley, R., 2000. $\mathrm{CO}_{2}$ storage as carbonate minerals. (PH3/17). United Kingdom: International Energy Agency.

O'Connor, W.K., Dahlin, D.C., Nilsen, D.N., Rush, G., Walters, R.P. \& Turner, P.C. 2001. Carbon Dioxide Sequestration by Direct Mineral Carbonation: Results from Recent Studies and Current Status.

O'Connor, W., Dahlin, D., Nilsen, D., Gerdemann, S., Rush, G., Penner, L., Walters, R., \& Turner, P., 2002. Continuing Studies on Direct Aqueous Mineral Carbonation for $\mathrm{CO}_{2}$ Sequestration. 27th International Technical Conf. on Coal Utilization \& Fuel Systems. March 4-7.

O'Connor, W., Dahlin, D., Nilsen, D., Walters, R., \& Turner, P., 2000. Carbon dioxide sequestration by direct mineral carbonation with carbonic acid. 25th International Technical Conference on Coal Utilization \& Fuel Systems. March 6-9.

O'Connor, W., Dahlin, D., Rush, G., Gerdemann, S., Penner, L., \& Nilsen, D., 2005. Mineral Availability, Pretreatment, Reaction Paramaetrics, and Process Studies. (Final Report). United States: Office of Process Development: National Energy Technology Laboratory.

Olajire, A.A. 2013. A review of mineral carbonation technology in sequestration of $\mathrm{CO}_{2}$. Journal of Petroleum Science and Engineering. 109:364-392.

Papoulias, S.A. \& Grossmann, I.E. 1983. A structural optimization approach in process synthesis-II. Computers \& Chemical Engineering. 7(6):707-721.

Park, A.A. \& Fan, L. 2004. $\mathrm{CO}_{2}$ mineral sequestration: physically activated dissolution of serpentine and pH swing process. Chemical Engineering Science. 59(22):5241-5247.

Park, A.A., Jadhav, R. \& Fan, L. 2003. $\mathrm{CO}_{2}$ mineral sequestration: chemically enhanced aqueous carbonation of serpentine. The Canadian Journal of Chemical Engineering. 81(3-4):885-890. 
Picot, J., Cassard, D., Maldan, F., Greffie, C., \& Bodenan, F., 2010. Worldwide potential for ex-situ mineral carbonation (Carmex project). International Conference on Greenhouse Gas Control Technologies. 19-23 September.

PRé. 2015. SimaPro Database Manual Methods Library. PRé.

Prigiobbe, V. \& Mazzotti, M. 2011. Dissolution of olivine in the presence of oxalate, citrate, and $\mathrm{CO}_{2}$ at $90^{\circ} \mathrm{C}$ and $120^{\circ} \mathrm{C}$. Chemical Engineering Science. 66(24):6544-6554.

PWC 2012. The gas equation - An analysis of the natural gas industry potential in South Africa. South Africa: PWC.

Rackley, S. 2010. Carbon capture and storage. Gulf Professional Publishing.

Rebitser, G., Ekvall, T., Frischknecht, R., Hunkeler, D., Norris, G., Rydberg, T., Schmidt, W., Suh, S., et al. 2004. Life cycle assessment Part 1: Framework, goal and scope definition, inventory analysis, and applications. Environment International. 30:701-720.

Romão, I., Nduagu, E., Fagerlund, J., Gando-Ferreira, L., \& Zevenhoven, R., 2012. $\mathrm{CO}_{2}$ fixation using magnesium silicate minerals. Part 2: Energy efficiency and integration with iron-and steelmaking. Energy. 41:203-211.

Sanna, A., Uibu, M., Caramanna, G., Kuusik, R., \& Maroto-Valer, M., 2014b. A review of mineral carbonation technologies to sequester $\mathrm{CO}_{2}$. Chemical Society Reviews. 43(23):8049-8080.

Sanna, A., Hall, M.R. \& Maroto-Valer, M. 2012. Post-processing pathways in carbon capture and storage by mineral carbonation (CCSM) towards the introduction of carbon neutral materials. Energy \& Environmental Science. 5(7):7781-7796.

Sanna, A., Lacinska, A., Styles, M. \& Maroto-Valer, M.M. 2014a. Silicate rock dissolution by ammonium bisulphate for $\mathrm{pH}$ swing mineral $\mathrm{CO}_{2}$ sequestration. Fuel Processing Technology. 120:128-135.

Sanna, A. \& Maroto-Valer, M.M. 2014c. $\mathrm{CO}_{2}$ Sequestration Using a Novel Na-salts pH Swing Mineral Carbonation Process. Energy Procedia. 63:5897-5903.

DOI:http://dx.doi.org/10.1016/j.egypro.2014.11.624.

Sinnott, R.K. \& Towler, G. 2008. Chemical Engineering Design: Principles, Practice and Economics of Plant and Process Design. Elsevier.

Slotte, M., Romão, I. \& Zevenhoven, R. 2013. Integration of a pilot-scale serpentinite carbonation process with an industrial lime kiln. Energy. 62:142-149.

Smith, J.M., Van Ness, H.C. \& Abbott, M.M. 2005. Introduction to chemical engineering thermodynamics. Boston: McGraw-Hill; 7th ed.

South African Carbon Capture \& Storage 2013. SACCS Charter Purpose and Objectives. South Africa: SACCS. 
Svensson, R., Odenberger, M., Johnsson, F., \& Strömberg, L., 2004. Transport systems for $\mathrm{CO}_{2}$ - application to carbon capture and storage. Energy Conversion and Management. 45:2343-2353.

Svrcek, W., Mahoney, D., \& Young, B.,. 2006. A Real-Time Approach to Process Control. 2nd ed. England: John Wiley \& Sons.

Teir, S., Eloneva, S., \& Zevenhoven, R., 2005. Production of precipitated calcium carbonate from calcium silicates and carbon dioxide. Energy Conversion and Management. 46(1819):2954-2979.

Teir, S., Kuusik, R., Fogelholm, C., \& Zevenhoven, R., 2007b. Production of magnesium carbonates from serpentine for long-term storage of $\mathrm{CO}_{2}$. International Journal of Mineral Processing. 85(1-3):1-15.

Teir, S., Revitzer, H., Eloneva, S., Fogelholm, C., \& Zevenhoven, R., 2007a. Dissolution of natural serpentinite in mineral and organic acids. International Journal of Mineral Processing. 83(1-2):36-46.

Teir, S., Zevenhoven, R., Eloneva, S., Lepistö, T., Savolahti, J., Aatos, S., Kontinen, A., Sorjonen-Ward, P., et al. 2006. $\mathrm{CO}_{2}$ emissions: mineral carbonation and Finnish pulp and paper industry $\left(\mathrm{CO}_{2}\right.$ Nordic Plus) and Use of serpentinites in energy and metal industry (ECOSERP).

Teir, S., Eloneva, S., Fogelholm, C. \& Zevenhoven, R. 2009. Fixation of carbon dioxide by producing hydromagnesite from serpentinite. Applied Energy. 86(2):214-218.

DOI:http://dx.doi.org/10.1016/j.apenergy.2008.03.013.

Thekdi, A.,. 2007. Improving Process Heating System Performance: A Sourcebook for Industry. 2nd ed. United States: US Department of Energy.

Thieme, C.,. 2005. "Sodium Carbonates", Ullmann's Encyclopedia of Industrial Chemistry. 7th ed. 2005: Wiley-VCH.

Viljoen, J.H.A., Stapelberg, F.D.J., \& Cloete, M., 2010. Technical Report on the Geological Storage of Carbon Dioxide in South Africa. South Africa: Council for Geoscience.

Vogeli, J., Reid, D., Becker, M., Broadhurst, J. \& Franzidis, J. 2011. Investigation of the potential for mineral carbonation of PGM tailings in South Africa. Minerals Engineering. 24(12):1348-1356.

Wang, X. \& Maroto-Valer, M.M. 2011. Dissolution of serpentine using recyclable ammonium salts for $\mathrm{CO}_{2}$ mineral carbonation. Fuel. 90(3):1229-1237.

Wendt, C., Butt, D., Lackner, K., \& Ziock, H., 1998. Thermodynamic considerations of using chlorides to accelerate the carbonate formation from magnesium silicates. International Conference on Greenhouse Gas Control Technologies. Aug 30 - Sept 2. Switzerland: . 
Westphal, G., Kristen, G., Wegener, W., Ambatiello, P., Helmut, G., Epron, B., Bonal, C., \& Steinhauser, G.,. 2005. "Sodium Chloride", Ullmann's Encyclopedia of Industrial Chemistry. 7th ed. Germany: Wiley-VCH.

World Resources Institute (WRI) 2008. Guideline for Carbon Dioxide Capture, Transport, and Storage. United States: World Resources Institute.

Zevenhoven, R., \& Kavaliauskaite, I., 2004. Mineral Carbonation for Long-term CO2 Storage: an Exergy Analysis. International Journal of Thermodynamics. 7(1):23-31.

Zevenhoven, R., \& Kohlmann, J., 2002. Direct Dry Mineral Carbonation for $\mathrm{CO}_{2}$ Emissions Reduction in Finland. International Technical Conference on Coal Utilization \& Fuel Systems. March 4-7.

Zevenhoven, R., \& Teir, S., 2004. Long-Term Storage of $\mathrm{CO}_{2}$ as Magnesium Carbonate in Finland. 3rd Annual Conference on Carbon Capture and Sequestration. May 3-6.

Zevenhoven, R., Fagerlund, J. \& Songok, J.K. 2011. $\mathrm{CO}_{2}$ mineral sequestration: developments toward large-scale application. Greenhouse Gases: Science and Technology. 1(1):48-57. 


\section{Appendix A: Initialization Data for AspenPlus Simulations}

\section{A.1 Ammonium Salts Process}

Table A-1: Initialization data for Aspen modelling of ammonium salts process

\begin{tabular}{|c|c|c|c|c|}
\hline Block ID & $\begin{array}{l}\text { Aspen } \\
\text { Model }\end{array}$ & $\begin{array}{l}\text { Property } \\
\text { Method }\end{array}$ & Purpose & Input Specifications \\
\hline HEATER-1 & Heater & ELECNRTL & $\begin{array}{l}\text { Preheats the } \\
\text { feed stream of } \\
\text { mineral prior to } \\
\text { leaching. }\end{array}$ & $\begin{array}{l}\text { Flash Type: Temperature-Pressure } \\
\text { Temperature: } 90^{\circ} \mathrm{C} \\
\text { Pressure: } 1 \mathrm{~atm}\end{array}$ \\
\hline HEATER-2 & Heater & ELECNRTL & $\begin{array}{l}\text { Preheats the } \\
\text { feed stream of } \\
\text { ammonium } \\
\text { bisulphate prior } \\
\text { to leaching. }\end{array}$ & $\begin{array}{l}\text { Flash Type: Temperature-Pressure } \\
\text { Temperature: } 90^{\circ} \mathrm{C} \\
\text { Pressure: } 1 \text { atm }\end{array}$ \\
\hline SIO2-SEP & Sep & GLOBAL & $\begin{array}{l}\text { Separates } \\
\text { aqueous } \\
\text { solution from } \\
\text { silica and } \\
\text { tailings residue. }\end{array}$ & $\begin{array}{l}\text { Outlet Stream: SIO2-PYR } \\
\text { Split Fraction: } \\
\text { PYROXENE }=1 \\
\text { SIO2 }=1\end{array}$ \\
\hline MIN-DIS & RStoich & ELECNRTL & $\begin{array}{l}\text { The ammonium } \\
\text { salts-based } \\
\text { extraction of } \\
\text { magnesium } \\
\text { from pyroxene } \\
\text { to produce } \\
\text { magnesium } \\
\text { chloride. }\end{array}$ & $\begin{array}{l}\text { Flash Type: Temperature-Pressure } \\
\text { Temperature: } 90{ }^{\circ} \mathrm{C} \\
\text { Pressure: } 1 \mathrm{~atm} \\
\text { Reactions: Conversion }=30 \%, 50 \%, 90 \% \\
\mathrm{MgSiO}_{3}+2 \mathrm{NH}_{4}^{+}+2 \mathrm{HSO}_{4}^{-} \rightarrow \mathrm{MgSO}_{4}+\mathrm{SiO}_{2}+\left(\mathrm{NH}_{4}\right)_{2} \mathrm{SO}_{4}+\mathrm{H}_{2} \mathrm{O}\end{array}$ \\
\hline HEATER-3 & Heater & ELECNRTL & $\begin{array}{l}\text { Cools LEACH- } \\
1 \text { stream to PH- } \\
\text { ADJ reaction } \\
\text { temperature. }\end{array}$ & $\begin{array}{l}\text { Flash Type: Temperature-Pressure } \\
\text { Temperature: } 25^{\circ} \mathrm{C} \\
\text { Pressure: } 1 \mathrm{~atm}\end{array}$ \\
\hline PH-ADJ & RStoich & ELECNRTL & $\begin{array}{l}\text { Adjust solution } \\
\mathrm{pH} \text { from acidic } \\
\text { to alkaline } \\
\text { conditions. }\end{array}$ & 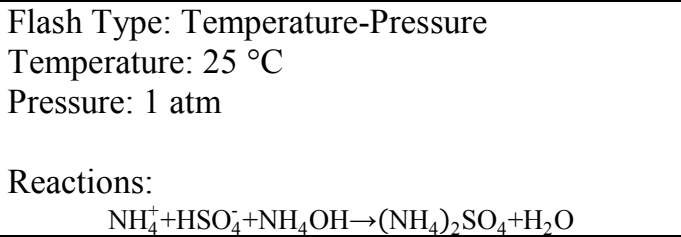 \\
\hline HEATER-4 & Heater & ELECNRTL & $\begin{array}{l}\text { Preheats } \mathrm{pH} \\
\text { adjusted leach } \\
\text { solution to } \\
\text { carbonation } \\
\text { reaction } \\
\text { conditions. } \\
\end{array}$ & $\begin{array}{l}\text { Flash Type: Temperature-Pressure } \\
\text { Temperature: } 80^{\circ} \mathrm{C} \\
\text { Pressure: } 1 \mathrm{~atm}\end{array}$ \\
\hline HEATER-5 & Heater & ELECNRTL & $\begin{array}{l}\text { Preheats } \\
\text { ammonium } \\
\text { bicarbonate } \\
\text { stream to } \\
\text { carbonation } \\
\text { reaction } \\
\text { conditions. }\end{array}$ & $\begin{array}{l}\text { Flash Type: Temperature-Pressure } \\
\text { Temperature: } 80^{\circ} \mathrm{C} \\
\text { Pressure: } 1 \text { atm }\end{array}$ \\
\hline
\end{tabular}




\begin{tabular}{|c|c|c|c|c|}
\hline MIN-CARB & RStoich & ELECNRTL & $\begin{array}{l}\text { The stable } \\
\text { carbonate } \\
\text { compound is } \\
\text { produced from } \\
\text { magnesium } \\
\text { sulphate. }\end{array}$ & $\begin{array}{l}\text { Flash Type: Temperature-Pressure } \\
\text { Temperature: } 80{ }^{\circ} \mathrm{C} \\
\text { Pressure: } 1 \mathrm{~atm} \\
\text { Reactions: Conversion }=90 \%(\text { All Cases) } \\
\mathrm{Mg}^{2+}+\mathrm{SO}_{4}^{2-}+2 \mathrm{NH}_{4}^{+}+2 \mathrm{HCO}_{3}^{-}+2 \mathrm{H}_{2} \mathrm{O} \rightarrow \mathrm{MgCO}_{3}+\left(\mathrm{NH}_{4}\right)_{2} \mathrm{SO}_{4}+\mathrm{CO}_{2}\end{array}$ \\
\hline CARB-SEP & Sep & GLOBAL & $\begin{array}{l}\text { Separates out } \\
\text { stable carbonate } \\
\text { compound from } \\
\text { sulphate-rich } \\
\text { solution }\end{array}$ & $\begin{array}{l}\text { Outlet Stream: MGCO3 } \\
\text { Split Fraction: } \\
\text { MGCO3 = } 1\end{array}$ \\
\hline CO2-VENT & Sep & GLOBAL & $\begin{array}{l}\text { Removes } \mathrm{CO}_{2} \\
\text { from sulphate- } \\
\text { rich solution. }\end{array}$ & $\begin{array}{l}\text { Outlet Stream: CO2 } \\
\text { Split Fraction: } \\
\mathrm{CO} 2=1\end{array}$ \\
\hline HEATER-7 & Heater & ELECNRTL & $\begin{array}{l}\text { Cools } \mathrm{CO}_{2} \\
\text { stream to } \\
\text { capture } \\
\text { conditions. } \\
\end{array}$ & $\begin{array}{l}\text { Flash Type: Temperature-Pressure } \\
\text { Temperature: } 10^{\circ} \mathrm{C} \\
\text { Pressure: } 1 \mathrm{~atm}\end{array}$ \\
\hline HEATER-9 & Heater & ELECNRTL & $\begin{array}{l}\text { Heats up } \\
\text { sulphate-rich } \\
\text { solution to } \\
\text { evaporate water. }\end{array}$ & $\begin{array}{l}\text { Flash Type: Temperature-Pressure } \\
\text { Temperature: } 120^{\circ} \mathrm{C} \\
\text { Pressure: } 1 \mathrm{~atm}\end{array}$ \\
\hline SEP-01 & Sep & GLOBAL & $\begin{array}{l}\text { Removes } \\
\text { evaporated } \\
\text { water from } \\
\text { sulphate-rich } \\
\text { stream. }\end{array}$ & $\begin{array}{l}\text { Outlet Stream: EVAP-PRD } \\
\text { Split Fraction: } \\
\text { WATER }=0.99\end{array}$ \\
\hline HEATER-X & Heater & ELECNRTL & $\begin{array}{l}\text { Preheats } \\
\text { sulphate-rich } \\
\text { stream to } \\
\text { thermal } \\
\text { decomposition } \\
\text { reaction } \\
\text { conditions. }\end{array}$ & $\begin{array}{l}\text { Flash Type: Temperature-Pressure } \\
\text { Temperature: } 300{ }^{\circ} \mathrm{C} \\
\text { Pressure: } 1 \text { atm }\end{array}$ \\
\hline EVAP & RStoich & ELECNRTL & $\begin{array}{l}\text { Converts } \\
\text { ammonium } \\
\text { sulphate to } \\
\text { ammonium } \\
\text { bisulphate and } \\
\text { ammonia via } \\
\text { thermal } \\
\text { decomposition. }\end{array}$ & $\begin{array}{l}\text { Flash Type: Temperature-Pressure } \\
\text { Temperature: } 300{ }^{\circ} \mathrm{C} \\
\text { Pressure: } 1 \text { atm } \\
\text { Reactions: } \\
\left(\mathrm{NH}_{4}\right)_{2} \mathrm{SO}_{4} \rightarrow \mathrm{NH}_{4} \mathrm{HSO}_{4}+\mathrm{NH}_{3}\end{array}$ \\
\hline HEATER-8 & Heater & ELECNRTL & $\begin{array}{l}\text { Cools } \\
\text { evaporated } \\
\text { steam prior to } \\
\text { carbon capture. }\end{array}$ & $\begin{array}{l}\text { Flash Type: Temperature-Pressure } \\
\text { Temperature: } 10^{\circ} \mathrm{C} \\
\text { Pressure: } 1 \mathrm{~atm}\end{array}$ \\
\hline HEATER-6 & Heater & ELECNRTL & $\begin{array}{l}\text { Cools } \\
\text { decomposition } \\
\text { product stream } \\
\text { DEC-PRD. }\end{array}$ & $\begin{array}{l}\text { Flash Type: Temperature-Pressure } \\
\text { Temperature: } 90^{\circ} \mathrm{C} \\
\text { Pressure: } 1 \text { atm }\end{array}$ \\
\hline SEP-02 & Sep & GLOBAL & $\begin{array}{l}\text { Separates } \\
\text { ammonia from } \\
\text { ammonium } \\
\text { bisulphate. }\end{array}$ & $\begin{array}{l}\text { Outlet Stream: CAP-NH3 } \\
\text { Split Fraction: } \\
\text { NH3 = } 1\end{array}$ \\
\hline CO2-CAPT & RStoich & ELECNRTL & $\begin{array}{l}\text { Captures } \mathrm{CO}_{2} \\
\text { from producing } \\
\text { ammonium } \\
\text { bicarbonate. }\end{array}$ & $\begin{array}{l}\text { Flash Type: Temperature-Pressure } \\
\text { Temperature: } 10^{\circ} \mathrm{C} \\
\text { Pressure: } 1 \mathrm{~atm}\end{array}$ \\
\hline
\end{tabular}




\begin{tabular}{|c|c|c|l|l|}
\hline & & Geactions: \\
\hline H2O-SEP & Sep & Global & $\begin{array}{l}\text { Separates } \\
\text { ammonium } \\
\text { bicarbonate } \\
\text { used in } \\
\text { carbonation. }\end{array}$ & $\begin{array}{l}\text { Outlet Stream: } \mathrm{NH} 3 \\
\text { Split Fraction: } \\
\text { WATER }=0.792 \\
\text { NH4HCO3 } 0\end{array}$ \\
\hline SPLIT-01 & Fsplit & GLOBAL & $\begin{array}{l}\text { Provides water } \\
\text { for use in } \mathrm{CO}_{2} \\
\text { capture. }\end{array}$ & $\begin{array}{l}\text { Stream: CAP-H2O } \\
\text { Split Fraction: } 0.3\end{array}$ \\
\hline SPLIT-2 & Fsplit & GLOBAL & $\begin{array}{l}\text { Recycles } \\
\text { unreacted } \\
\text { pyroxene to } \\
\text { dissolution } \\
\text { reactor. }\end{array}$ & $\begin{array}{l}\text { Stream: SIO2-PRD } \\
\text { Split Fraction: } 0.2\end{array}$ \\
\hline REC-SPLT & Fsplit & GLOBAL & $\begin{array}{l}\text { Recycles } \\
\text { regenerated acid } \\
\text { to dissolution } \\
\text { reactor. }\end{array}$ & $\begin{array}{l}\text { Stream: BIS-PRD } \\
\text { Split Fraction: } 0.2\end{array}$ \\
\hline MIX-1 & Mixer & ELECNRTL & $\begin{array}{l}\text { Produces } \\
\text { solution. }\end{array}$ & $\begin{array}{l}\text { Pressure: } 1 \text { atm } \\
\text { Valid Phases: Vapor-Liquid }\end{array}$ \\
\hline
\end{tabular}

\section{A.2 Lackner's Multi-stage $\mathrm{HCl}$ (AAU) Process}

Table A-2: Initialization data for Aspen Modelling of Lackner's multi-stage process

\begin{tabular}{|c|c|c|c|c|}
\hline Block ID & $\begin{array}{l}\text { Aspen } \\
\text { Model }\end{array}$ & $\begin{array}{l}\text { Property } \\
\text { Method }\end{array}$ & Purpose & Input Specifications \\
\hline HEATER-1 & Heater & ELECNRTL & $\begin{array}{l}\text { Preheats the } \\
\text { feed stream of } \\
\text { mineral prior to } \\
\text { leaching. }\end{array}$ & $\begin{array}{l}\text { Flash Type: Temperature-Pressure } \\
\text { Temperature: } 70^{\circ} \mathrm{C} \\
\text { Pressure: } 1 \mathrm{~atm}\end{array}$ \\
\hline HEATER-2 & Heater & ELECNRTL & $\begin{array}{l}\text { Preheats the } \\
\text { feed stream of } \\
\text { hydrochloric } \\
\text { acid prior to } \\
\text { leaching. }\end{array}$ & $\begin{array}{l}\text { Flash Type: Temperature-Pressure } \\
\text { Temperature: } 70^{\circ} \mathrm{C} \\
\text { Pressure: } 1 \mathrm{~atm}\end{array}$ \\
\hline MIN-DIS & RStoich & ELECNRTL & $\begin{array}{l}\text { The acid-based } \\
\text { extraction of } \\
\text { magnesium } \\
\text { from pyroxene } \\
\text { to produce } \\
\text { magnesium } \\
\text { chloride. }\end{array}$ & $\begin{array}{l}\text { Flash Type: Temperature-Pressure } \\
\text { Temperature: } 70^{\circ} \mathrm{C} \\
\text { Pressure: } 1 \mathrm{~atm} \\
\text { Reactions: } \\
\quad \mathrm{MgSiO}_{3}+2 \mathrm{H}^{+}+2 \mathrm{Cl}^{-}+5 \mathrm{H}_{2} \mathrm{O} \rightarrow \mathrm{MgCl}_{2} \cdot 6 \mathrm{H}_{2} \mathrm{O}+\mathrm{SiO}_{2}\end{array}$ \\
\hline SPLIT-1 & Fsplit & GLOBAL & $\begin{array}{l}\text { Recycles } \\
\text { unreacted } \\
\text { pyroxene to } \\
\text { dissolution } \\
\text { reactor. }\end{array}$ & $\begin{array}{l}\text { Stream: PYR-REC } \\
\text { Split Fraction: } 0.9\end{array}$ \\
\hline
\end{tabular}




\begin{tabular}{|c|c|c|c|c|}
\hline SEP-1 & Sep & GLOBAL & $\begin{array}{l}\text { Separates } \\
\text { aqueous } \\
\text { magnesium } \\
\text { chloride from } \\
\text { silica and } \\
\text { tailings residue. }\end{array}$ & $\begin{array}{l}\text { Outlet Stream: PYR-SIO2 } \\
\text { Split Fraction: } \\
\text { PYROXENE }=1 \\
\text { SIO2 }=1\end{array}$ \\
\hline CONV & RStoich & ELECNRTL & $\begin{array}{l}\text { Magnesium } \\
\text { chloride } \\
\text { conversion and } \\
\text { regeneration of } \\
\text { hydrochloric } \\
\text { acid. }\end{array}$ & $\begin{array}{l}\text { Flash Type: Temperature-Pressure } \\
\text { Temperature: } 150^{\circ} \mathrm{C} \\
\text { Pressure: } 1 \mathrm{~atm} \\
\text { Reactions: } \\
\quad \mathrm{MgCl}_{2} \cdot 6 \mathrm{H}_{2} \mathrm{O} \rightarrow \mathrm{MgOHCl}+\mathrm{HCl}+5 \mathrm{H}_{2} \mathrm{O}\end{array}$ \\
\hline SEP-5 & Sep & GLOBAL & $\begin{array}{l}\text { Removes } \\
\text { regenerated acid } \\
\text { from product } \\
\text { stream. }\end{array}$ & $\begin{array}{l}\text { Outlet Stream: ACID } \\
\text { Split Fraction: } \\
\text { HCL }=1 \\
\text { WATER }=0.8\end{array}$ \\
\hline HEATER-4 & Heater & ELECNRTL & $\begin{array}{l}\text { Cools and } \\
\text { condenses } \\
\text { regenerated acid } \\
\text { stream. }\end{array}$ & $\begin{array}{l}\text { Flash Type: Temperature-Pressure } \\
\text { Temperature: } 70^{\circ} \mathrm{C} \\
\text { Pressure: } 1 \mathrm{~atm}\end{array}$ \\
\hline REPART & RStoich & ELECNRTL & $\begin{array}{l}\text { Reforms } \\
\text { magnesium } \\
\text { chloride } \\
\text { hydroxide, } \\
\text { producing } \\
\text { magnesium } \\
\text { hydroxide. }\end{array}$ & $\begin{array}{l}\text { Flash Type: Temperature-Pressure } \\
\text { Temperature: } 25^{\circ} \mathrm{C} \\
\text { Pressure: } 1 \mathrm{~atm} \\
\text { Reactions: } \\
\quad 2 \mathrm{MgOHCl} \rightarrow \mathrm{Mg}(\mathrm{OH})_{2}+\mathrm{MgCl}_{2}\end{array}$ \\
\hline SPLIT-2 & Fsplit & GLOBAL & $\begin{array}{l}\text { Recycles } \\
\text { hydrochloric } \\
\text { acid to the } \\
\text { dissolution unit. }\end{array}$ & $\begin{array}{l}\text { Stream: HCL-REC } \\
\text { Split Fraction: } 0.9\end{array}$ \\
\hline SEP-2 & Sep & GLOBAL & $\begin{array}{l}\text { Removes solid } \\
\text { magnesium } \\
\text { hydroxide from } \\
\text { magnesium } \\
\text { chloride } \\
\text { solution }\end{array}$ & $\begin{array}{l}\text { Outlet Stream: H2O-2 } \\
\text { Split Fraction: } \\
\text { MGCL2 }=1 \\
\text { WATER }=1\end{array}$ \\
\hline MIN-CARB & RStoich & UNIQUAC & $\begin{array}{l}\text { The stable } \\
\text { carbonate } \\
\text { compound is } \\
\text { produced from } \\
\text { magnesium } \\
\text { hydroxide. }\end{array}$ & $\begin{array}{l}\text { Flash Type: Temperature-Pressure } \\
\text { Temperature: } 680 \mathrm{~K} \\
\text { Pressure: } 1 \mathrm{~atm} \\
\text { Reactions: } \\
\mathrm{Mg}(\mathrm{OH})_{2}+\mathrm{CO}_{2} \rightarrow \mathrm{MgCO}_{3}+\mathrm{H}_{2} \mathrm{O}\end{array}$ \\
\hline SEP-3 & Sep & GLOBAL & $\begin{array}{l}\text { Removes water } \\
\text { from solid } \\
\text { magnesium } \\
\text { carbonate } \\
\text { product. }\end{array}$ & $\begin{array}{l}\text { Outlet Stream: } \mathrm{H} 2 \mathrm{O} \\
\text { Split Fraction: } \\
\text { WATER }=1\end{array}$ \\
\hline
\end{tabular}




\section{A.3 Åbo Akademi University (AAU) Process}

Table A-3: Initialization data for Aspen Modelling of Ảbo Akademi University process

\begin{tabular}{|c|c|c|c|c|}
\hline Block ID & $\begin{array}{l}\text { Aspen } \\
\text { Model }\end{array}$ & $\begin{array}{l}\text { Property } \\
\text { Method }\end{array}$ & Purpose & Input Specifications \\
\hline HEATER-0 & Heater & UNIQUAC & $\begin{array}{l}\text { Preheats } \\
\text { pyroxene feed } \\
\text { stream of } \\
\text { mineral prior to } \\
\text { leaching. }\end{array}$ & $\begin{array}{l}\text { Flash Type: Temperature-Pressure } \\
\text { Temperature: } 400^{\circ} \mathrm{C} \\
\text { Pressure: } 1 \text { atm }\end{array}$ \\
\hline AS-REAC & RStoich & UNIQUAC & $\begin{array}{l}\text { The ammonium } \\
\text { sulphate-based } \\
\text { extraction of } \\
\text { magnesium } \\
\text { from pyroxene } \\
\text { to produce } \\
\text { magnesium } \\
\text { sulphate. }\end{array}$ & $\begin{array}{l}\text { Flash Type: Temperature-Pressure } \\
\text { Temperature: } 400{ }^{\circ} \mathrm{C} \\
\text { Pressure: } 1 \text { atm } \\
\text { Reactions: } \\
\mathrm{MgSiO}_{3}+\left(\mathrm{NH}_{4}\right)_{2} \mathrm{SO}_{4} \rightarrow \mathrm{MgSO}_{4}+\mathrm{SiO}_{2}+2 \mathrm{NH}_{3}+\mathrm{H}_{2} \mathrm{O}\end{array}$ \\
\hline SEP-1 & Sep & GLOBAL & $\begin{array}{l}\text { Separates } \\
\text { gaseous } \\
\text { ammonia and } \\
\text { steam from } \\
\text { solid products. }\end{array}$ & $\begin{array}{l}\text { Outlet Stream: NH3-H2O } \\
\text { Split Fraction: } \\
\text { NH3 }=1 \\
\text { WATER }=1\end{array}$ \\
\hline HEATER-1 & Heater & ELECNRTL & $\begin{array}{l}\text { Condenses } \\
\text { ammonia and } \\
\text { water prior to } \\
\text { precipitation. }\end{array}$ & $\begin{array}{l}\text { Flash Type: Temperature-Pressure } \\
\text { Temperature: } 25^{\circ} \mathrm{C} \\
\text { Pressure: } 1 \mathrm{~atm}\end{array}$ \\
\hline HEATER-3 & Heater & UNIQUAC & $\begin{array}{l}\text { Cools solids } \\
\text { stream prior to } \\
\text { mixing with } \\
\text { water. }\end{array}$ & $\begin{array}{l}\text { Flash Type: Temperature-Pressure } \\
\text { Temperature: } 40^{\circ} \mathrm{C} \\
\text { Pressure: } 1 \text { atm }\end{array}$ \\
\hline MIXER-1 & MIXER & ELECNRTL & $\begin{array}{l}\text { Mixes solids } \\
\text { stream with } \\
\text { water, } \\
\text { dissolving } \\
\text { magnesium } \\
\text { sulphate. } \\
\end{array}$ & $\begin{array}{l}\text { Pressure: } 1 \text { atm } \\
\text { Valid-Phases: Vapor-Liquid }\end{array}$ \\
\hline SEP-2 & Sep & GLOBAL & $\begin{array}{l}\text { Removes } \\
\text { dissolved } \\
\text { magnesium } \\
\text { sulphate } \\
\text { solution. }\end{array}$ & $\begin{array}{l}\text { Outlet Stream: MGSO-4 } \\
\text { Split Fraction: } \\
\text { SULPHATE }=1 \\
\text { MGSO4 }=1 \\
\text { NH3 }=1 \\
\text { WATER }=1 \\
\end{array}$ \\
\hline PRECIP & RStoich & ELECNRTL & $\begin{array}{l}\text { Precipitates } \\
\text { magnesium } \\
\text { hydroxide } \\
\text { through reaction } \\
\text { of ammonia, } \\
\text { water and } \\
\text { magnesium } \\
\text { sulphate. }\end{array}$ & $\begin{array}{l}\text { Flash Type: Temperature-Pressure } \\
\text { Temperature: } 40{ }^{\circ} \mathrm{C} \\
\text { Pressure: } 1 \text { atm } \\
\text { Reactions: } \\
\mathrm{Mg}^{2+}+2 \mathrm{NH}_{3}+2 \mathrm{H}_{2} \mathrm{O}+\mathrm{SO}_{4}^{2-} \rightarrow \mathrm{Mg}(\mathrm{OH})_{2}+\left(\mathrm{NH}_{4}\right)_{2} \mathrm{SO}_{4}\end{array}$ \\
\hline
\end{tabular}




\begin{tabular}{|c|c|c|c|c|}
\hline SPLIT-3 & FSplit & GLOBAL & $\begin{array}{l}\text { Recycles } \\
\text { pyroxene into } \\
\text { the extraction } \\
\text { unit. }\end{array}$ & $\begin{array}{l}\text { Stream: PYR-REC } \\
\text { Split Fraction: } 0.9\end{array}$ \\
\hline SEP-3 & Sep & GLOBAL & $\begin{array}{l}\text { Separates } \\
\text { magnesium } \\
\text { hydroxide from } \\
\text { sulphate stream. }\end{array}$ & $\begin{array}{l}\text { Outlet Stream: SULF-REC } \\
\text { Split Fraction: } \\
\text { SULFATE }=1 \\
\text { WATER }=1 \\
\end{array}$ \\
\hline HEATER-4 & Heater & ELECNRTL & $\begin{array}{l}\text { Evaporates } \\
\text { water from } \\
\text { ammonium } \\
\text { sulphate stream. }\end{array}$ & $\begin{array}{l}\text { Flash Type: Temperature-Pressure } \\
\text { Temperature: } 110^{\circ} \mathrm{C} \\
\text { Pressure: } 1 \mathrm{~atm}\end{array}$ \\
\hline HEATER-5 & Heater & UNIQUAC & $\begin{array}{l}\text { Preheats } \\
\text { magnesium } \\
\text { hydroxide } \\
\text { stream prior to } \\
\text { carbonation. } \\
\end{array}$ & $\begin{array}{l}\text { Flash Type: Temperature-Pressure } \\
\text { Temperature: } 450^{\circ} \mathrm{C} \\
\text { Pressure: } 1 \mathrm{~atm}\end{array}$ \\
\hline HEATER-6 & Heater & UNIQUAC & $\begin{array}{l}\text { Preheats carbon } \\
\text { dioxide stream } \\
\text { prior to } \\
\text { carbonation. }\end{array}$ & $\begin{array}{l}\text { Flash Type: Temperature-Pressure } \\
\text { Temperature: } 450^{\circ} \mathrm{C} \\
\text { Pressure: } 20 \text { bar }\end{array}$ \\
\hline MIN-CARB & RStoich & UNIQUAC & $\begin{array}{l}\text { Production of } \\
\text { stable carbonate } \\
\text { compound from } \\
\text { magnesium } \\
\text { hydroxide and } \\
\text { carbon dioxide. }\end{array}$ & $\begin{array}{l}\text { Flash Type: Temperature-Pressure } \\
\text { Temperature: } 450^{\circ} \mathrm{C} \\
\text { Pressure: } 20 \text { bar } \\
\text { Reactions: } \\
\quad \mathrm{Mg}(\mathrm{OH})_{2}+\mathrm{CO}_{2} \rightarrow \mathrm{MgCO}_{3}+\mathrm{H}_{2} \mathrm{O}\end{array}$ \\
\hline SEP-4 & Sep & GLOBAL & $\begin{array}{l}\text { Removes stable } \\
\text { carbonate } \\
\text { stream. }\end{array}$ & $\begin{array}{l}\text { Outlet Stream: CARB-STR } \\
\text { Split Fraction: } \\
\text { MGOH }=1 \\
\text { WATER }=1 \\
\text { CO2 }=1\end{array}$ \\
\hline SPLIT-1 & FSplit & GLOBAL & $\begin{array}{l}\text { Recycles } \\
\text { unreacted } \\
\text { components to } \\
\text { carbonation } \\
\text { reactor. }\end{array}$ & $\begin{array}{l}\text { Outlet Stream: CARB-H2O } \\
\text { Split Fraction: } 0.9\end{array}$ \\
\hline HEATER-2 & Heater & UNIQUAC & $\begin{array}{l}\text { Cools product } \\
\text { carbonate } \\
\text { stream. }\end{array}$ & $\begin{array}{l}\text { Flash Type: Temperature-Pressure } \\
\text { Temperature: } 30^{\circ} \mathrm{C} \\
\text { Pressure: } 20 \mathrm{bar}\end{array}$ \\
\hline SEP-6 & Sep & GLOBAL & $\begin{array}{l}\text { Recycles } \\
\text { unreacted } \\
\text { components to } \\
\text { carbonation } \\
\text { reactor. }\end{array}$ & $\begin{array}{l}\text { Outlet Stream: CARB-REC } \\
\text { Split Fraction: } \\
\text { MGOH }=1 \\
\mathrm{CO} 2=1\end{array}$ \\
\hline SEP-5 & Sep & GLOBAL & $\begin{array}{l}\text { Separates steam } \\
\text { from solid } \\
\text { ammonium } \\
\text { sulphate stream. }\end{array}$ & $\begin{array}{l}\text { Outlet Stream: STEAM } \\
\text { Split Fraction: } \\
\text { WATER = } 1\end{array}$ \\
\hline SPLIT-2 & FSplit & GLOBAL & $\begin{array}{l}\text { Recycles } \\
\text { regenerated } \\
\text { ammonium } \\
\text { sulphate to } \\
\text { extraction unit. }\end{array}$ & $\begin{array}{l}\text { Outlet Stream: AS-REC } \\
\text { Split Fraction: } 0.9\end{array}$ \\
\hline
\end{tabular}




\section{A.4 Mineral Acid pH-Swing Process}

Table A-4: Initialisation data for Aspen model of mineral acid pH swing process

\begin{tabular}{|c|c|c|c|c|}
\hline Block ID & $\begin{array}{l}\text { Aspen } \\
\text { Model }\end{array}$ & $\begin{array}{l}\text { Property } \\
\text { Method }\end{array}$ & Purpose & Input Specifications \\
\hline HEATER-1 & Heater & ELECNRTL & $\begin{array}{l}\text { Preheats the } \\
\text { feed stream of } \\
\text { mineral prior to } \\
\text { leaching. }\end{array}$ & $\begin{array}{l}\text { Flash Type: Temperature-Pressure } \\
\text { Temperature: } 70^{\circ} \mathrm{C} \\
\text { Pressure: } 1 \text { atm }\end{array}$ \\
\hline HEATER-2 & Heater & ELECNRTL & $\begin{array}{l}\text { Preheats the } \\
\text { feed stream of } \\
\text { hydrochloric } \\
\text { acid prior to } \\
\text { leaching. }\end{array}$ & $\begin{array}{l}\text { Flash Type: Temperature-Pressure } \\
\text { Temperature: } 70{ }^{\circ} \mathrm{C} \\
\text { Pressure: } 1 \mathrm{~atm}\end{array}$ \\
\hline HEATER-3 & Heater & ELECNRTL & $\begin{array}{l}\text { Heats acid } \\
\text { recycle stream } \\
\text { to reaction } \\
\text { temperature. }\end{array}$ & $\begin{array}{l}\text { Flash Type: Temperature-Pressure } \\
\text { Temperature: } 70^{\circ} \mathrm{C} \\
\text { Pressure: } 1 \mathrm{~atm}\end{array}$ \\
\hline MIN-DIS & RStoich & ELECNRTL & $\begin{array}{l}\text { The acid-based } \\
\text { extraction of } \\
\text { magnesium } \\
\text { from pyroxene } \\
\text { to produce } \\
\text { magnesium } \\
\text { chloride. }\end{array}$ & $\begin{array}{l}\text { Flash Type: Temperature-Pressure } \\
\text { Temperature: } 70{ }^{\circ} \mathrm{C} \\
\text { Pressure: } 1 \text { atm } \\
\text { Reactions: } \\
\mathrm{MgSiO}_{3}+2 \mathrm{H}^{+}+2 \mathrm{Cl}^{-} \rightarrow \mathrm{MgCl}_{2}+\mathrm{SiO}_{2}+\mathrm{H}_{2} \mathrm{O}\end{array}$ \\
\hline SEP-1 & Sep & GLOBAL & $\begin{array}{l}\text { Separates } \\
\text { aqueous } \\
\text { magnesium } \\
\text { chloride from } \\
\text { silica and } \\
\text { tailings residue. }\end{array}$ & $\begin{array}{l}\text { Outlet Stream: PYR-SIO2 } \\
\text { Split Fraction: } \\
\text { PYROXENE }=1 \\
\text { SIO2 }=1\end{array}$ \\
\hline SEP-2 & Sep & GLOBAL & $\begin{array}{l}\text { Removes } \\
\text { regenerated acid } \\
\text { from product } \\
\text { stream. }\end{array}$ & $\begin{array}{l}\text { Outlet Stream: ACID } \\
\text { Split Fraction: } \\
\text { HCL }=1 \\
\text { WATER }=1\end{array}$ \\
\hline SEP-3 & Sep & GLOBAL & $\begin{array}{l}\text { Removes } \\
\text { magnesium } \\
\text { carbonate } \\
\text { product from } \\
\text { process. }\end{array}$ & $\begin{array}{l}\text { Outlet Stream: MGCO3 } \\
\text { Split Fraction: } \\
\text { MGCO3 = } 1\end{array}$ \\
\hline PH-ADJ & RStoich & ELECNRTL & $\begin{array}{l}\text { Uses sodium } \\
\text { hydroxide to } \\
\text { adjust pH to } \\
\text { alkaline } \\
\text { conditions. }\end{array}$ & $\begin{array}{l}\text { Flash Type: Temperature-Pressure } \\
\text { Temperature: } 25^{\circ} \mathrm{C} \\
\text { Pressure: } 1 \text { atm } \\
\text { Reactions: } \\
\mathrm{NaOH}+\mathrm{HCl} \rightarrow \mathrm{NaCl}+\mathrm{H}_{2} \mathrm{O}\end{array}$ \\
\hline MIN-CARB & RStoich & ELECNRTL & $\begin{array}{l}\text { The stable } \\
\text { carbonate } \\
\text { compound is } \\
\text { produced from } \\
\text { magnesium } \\
\text { chloride. }\end{array}$ & $\begin{array}{l}\text { Flash Type: Temperature-Pressure } \\
\text { Temperature: } 20^{\circ} \mathrm{C} \\
\text { Pressure: } 1 \text { atm } \\
\text { Reactions: } \\
\mathrm{MgCl}_{2}+\mathrm{H}_{2} \mathrm{O}+\mathrm{CO}_{2} \rightarrow \mathrm{MgCO}_{3}+2 \mathrm{HCl} \\
\end{array}$ \\
\hline SPLIT-1 & Fsplit & GLOBAL & $\begin{array}{l}\text { Recycles } \\
\text { unreacted } \\
\text { pyroxene to }\end{array}$ & $\begin{array}{l}\text { Stream: PYR-REC } \\
\text { Split Fraction: } 0.8\end{array}$ \\
\hline
\end{tabular}




\begin{tabular}{|c|l|l|l|l|}
\hline & & & $\begin{array}{l}\text { dissolution } \\
\text { reactor. }\end{array}$ & \\
\hline SPLIT-2 & Fsplit & GLOBAL & $\begin{array}{l}\text { Recycles } \\
\text { regenerated acid } \\
\text { to dissolution } \\
\text { reactor. }\end{array}$ & $\begin{array}{l}\text { Stream: ACID-REC } \\
\text { Split Fraction: } 0.85\end{array}$ \\
\hline SPLIT-3 & Fsplit & GLOBAL & $\begin{array}{l}\text { Recycles } \\
\text { unreacted } \\
\text { magnesium } \\
\text { chloride } \\
\text { solution to } \\
\text { carbonation } \\
\text { reactor. }\end{array}$ & $\begin{array}{l}\text { Stream: SOL-REC } \\
\text { Split Fraction: } 0.85\end{array}$ \\
\hline
\end{tabular}

\section{A.5 Direct Aqueous Carbonation Process}

Table A-5: Initialisation data for Aspen model of direct aqueous process

\begin{tabular}{|c|c|c|c|c|}
\hline Block ID & $\begin{array}{l}\text { Aspen } \\
\text { Model }\end{array}$ & $\begin{array}{l}\text { Property } \\
\text { Method }\end{array}$ & Purpose & Input Specifications \\
\hline HEATER-1 & Heater & ELECNRTL & $\begin{array}{l}\text { Preheats the } \\
\text { feed stream of } \\
\text { mineral prior to } \\
\text { carbonation. }\end{array}$ & $\begin{array}{l}\text { Flash Type: Temperature-Pressure } \\
\text { Temperature: } 185^{\circ} \mathrm{C} \\
\text { Pressure: } 1 \mathrm{~atm}\end{array}$ \\
\hline HEATER-2 & Heater & UNIQUAC & $\begin{array}{l}\text { Preheats the } \\
\text { feed stream of } \\
\text { carbon dioxide } \\
\text { prior to } \\
\text { carbonation. }\end{array}$ & $\begin{array}{l}\text { Flash Type: Temperature-Pressure } \\
\text { Temperature: } 185^{\circ} \mathrm{C} \\
\text { Pressure: } 150 \mathrm{~atm}\end{array}$ \\
\hline HEATER-3 & Heater & ELECNRTL & $\begin{array}{l}\text { Heats feed water } \\
\text { stream prior to } \\
\text { carbonation. }\end{array}$ & $\begin{array}{l}\text { Flash Type: Temperature-Pressure } \\
\text { Temperature: } 185^{\circ} \mathrm{C} \\
\text { Pressure: } 150 \mathrm{~atm}\end{array}$ \\
\hline MIX-REAC & RStoich & ELECNRTL & $\begin{array}{l}\text { The production } \\
\text { of carbonic acid } \\
\text { from water and } \\
\text { carbon dioxide. }\end{array}$ & $\begin{array}{l}\text { Flash Type: Temperature-Pressure } \\
\text { Temperature: } 185^{\circ} \mathrm{C} \\
\text { Pressure: } 150 \mathrm{~atm} \\
\text { Reactions: } \mathrm{H}_{2} \mathrm{O}+\mathrm{CO}_{2} \rightarrow \mathrm{H}_{2} \mathrm{CO}_{3}\end{array}$ \\
\hline CRB-REAC & RStoich & ELECNRTL & $\begin{array}{l}\text { The production } \\
\text { of stable } \\
\text { carbonate from } \\
\text { reaction of } \\
\text { mineral with } \\
\text { carbonic acid }\end{array}$ & $\begin{array}{l}\text { Flash Type: Temperature-Pressure } \\
\text { Temperature: } 185^{\circ} \mathrm{C} \\
\text { Pressure: } 150 \mathrm{~atm} \\
\text { Reactions: } \\
\mathrm{MgSiO}_{3}+\mathrm{H}_{2} \mathrm{CO}_{3} \rightarrow \mathrm{MgCO}_{3}+\mathrm{SiO}_{3}+\mathrm{H}_{2} \mathrm{O}\end{array}$ \\
\hline SEP-1 & Sep & GLOBAL & $\begin{array}{l}\text { Separates solid } \\
\text { product chloride } \\
\text { from aqueous } \\
\text { solution. }\end{array}$ & $\begin{array}{l}\text { Outlet Stream: SOLIDS } \\
\text { Split Fraction: } \\
\text { PYROXENE }=1 \\
\text { SIO2 }=1 \\
\text { MGCO3 }=1\end{array}$ \\
\hline
\end{tabular}




\begin{tabular}{|c|l|l|l|l|}
\hline SEP-2 & Sep & GLOBAL & $\begin{array}{l}\text { Separates stable } \\
\text { carbonate } \\
\text { product }\end{array}$ & $\begin{array}{l}\text { Outlet Stream: SIO-PYR } \\
\text { Split Fraction: } \\
\text { PROX=1 } \\
\text { SIO2 }=1\end{array}$ \\
\hline SPLIT-1 & Fsplit & GLOBAL & $\begin{array}{l}\text { Recycles } \\
\text { unreacted } \\
\text { pyroxene to } \\
\text { dissolution } \\
\text { reactor. }\end{array}$ & $\begin{array}{l}\text { Stream: PYR-REC } \\
\text { Split Fraction: } 0.9\end{array}$ \\
\hline SPLIT-2 & Fsplit & GLOBAL & $\begin{array}{l}\text { Recycles } \\
\text { regenerated acid } \\
\text { to dissolution } \\
\text { reactor. }\end{array}$ & $\begin{array}{l}\text { Stream: ACID-REC } \\
\text { Split Fraction: } 0.95\end{array}$ \\
\hline
\end{tabular}




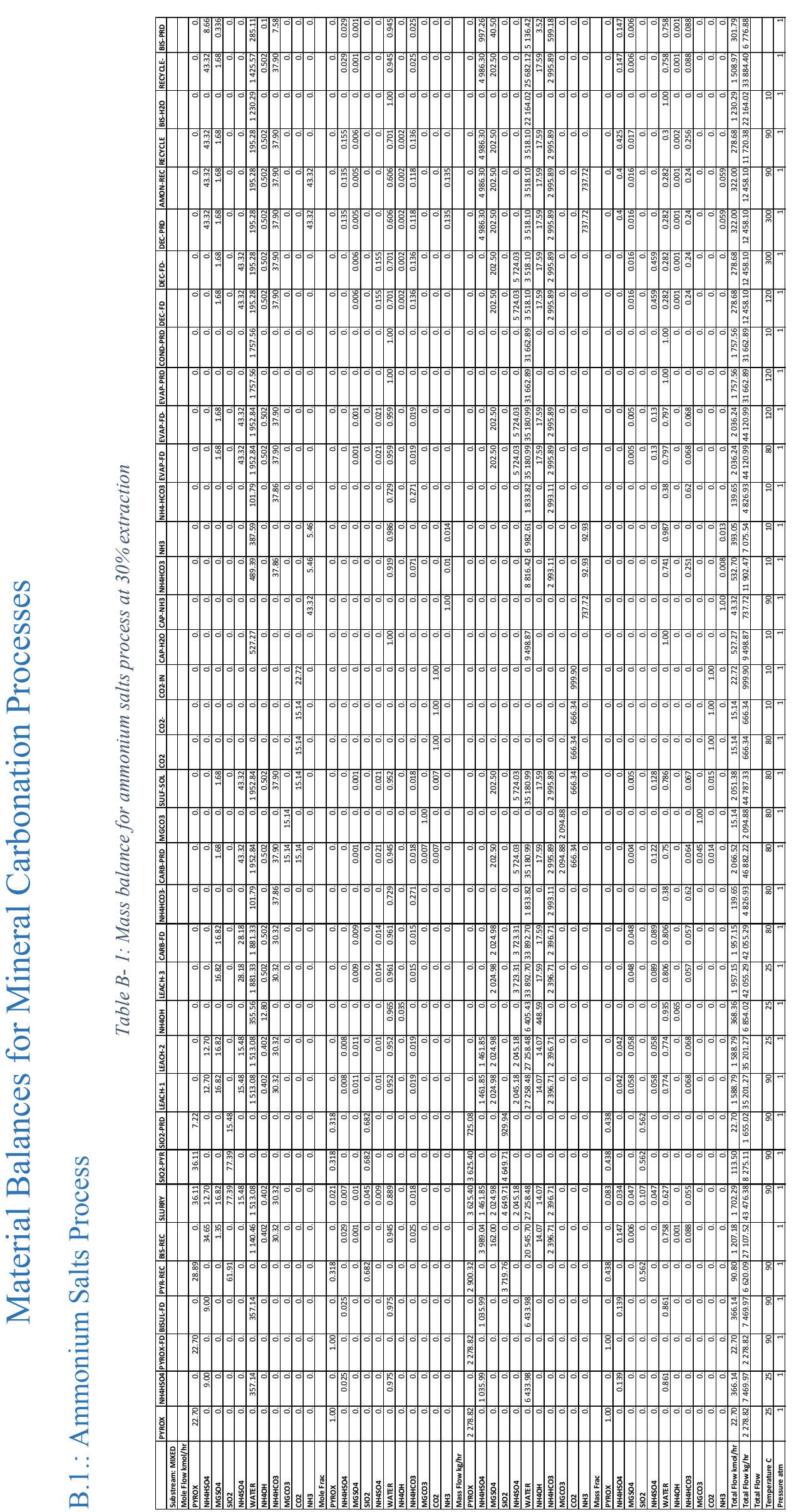




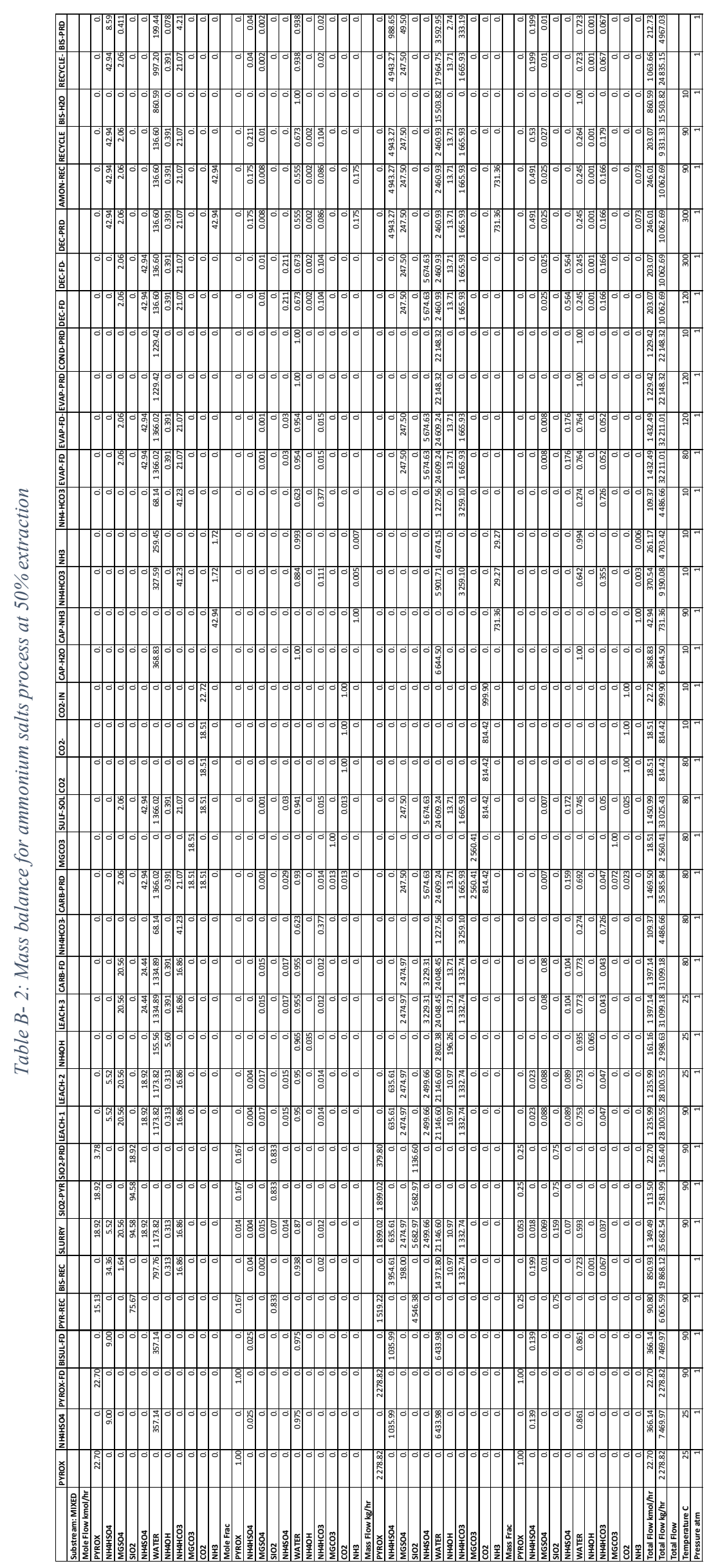




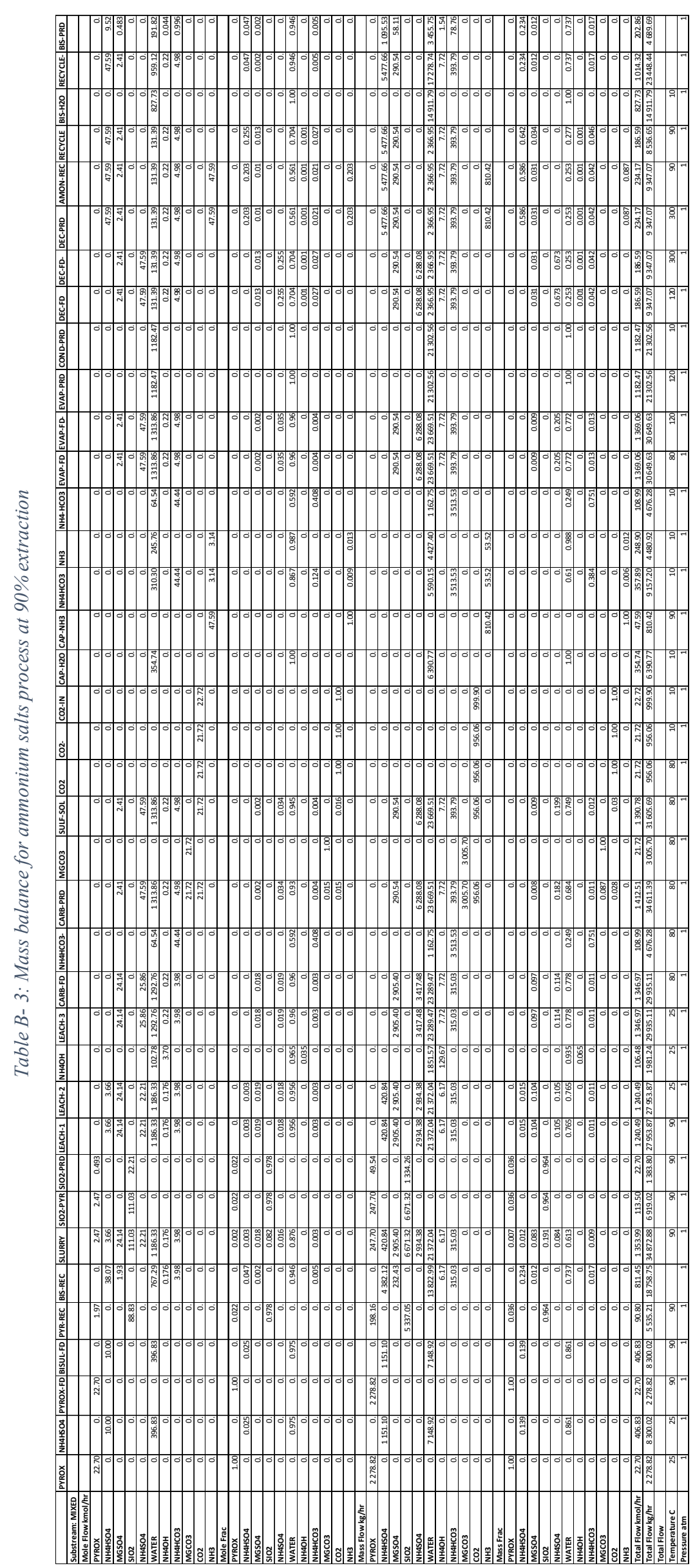




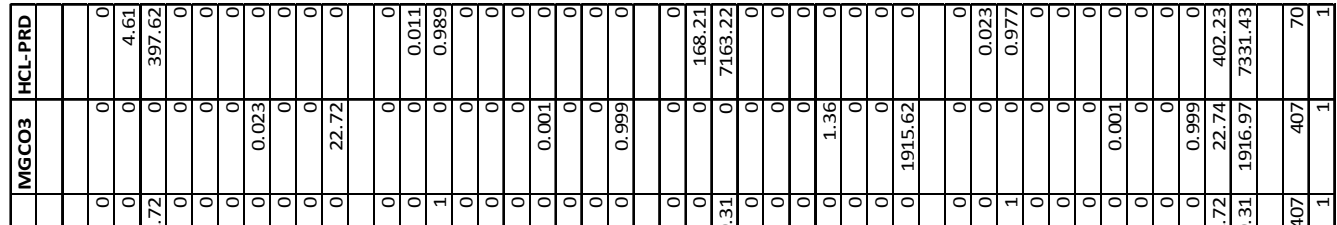

ㅇำ

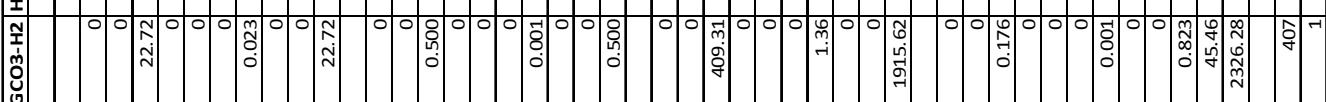

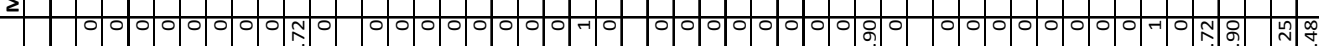
ฮิ

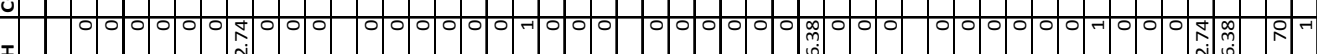

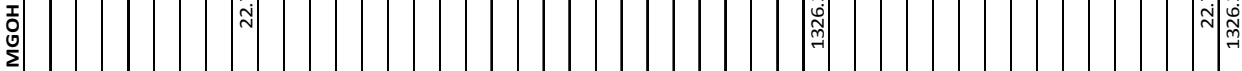

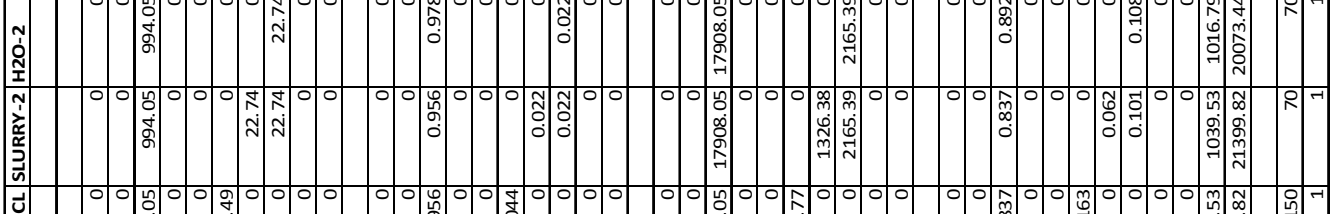

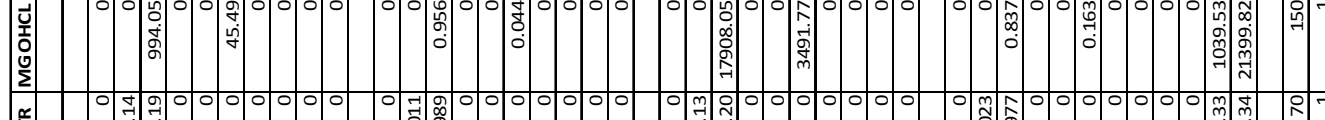

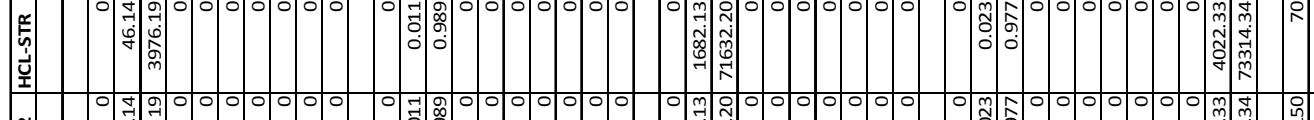

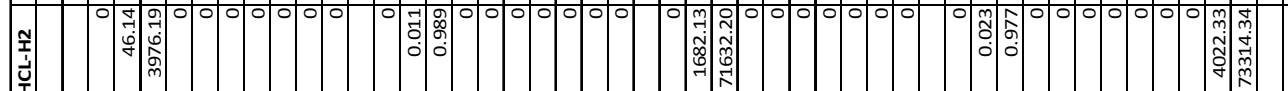

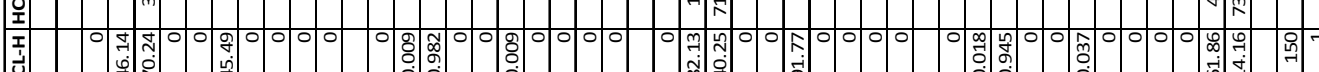

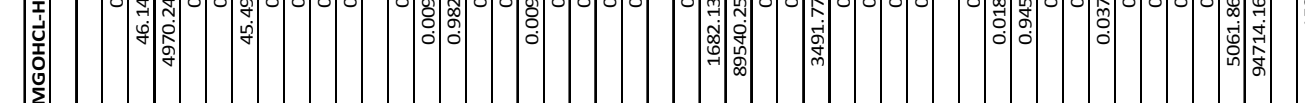

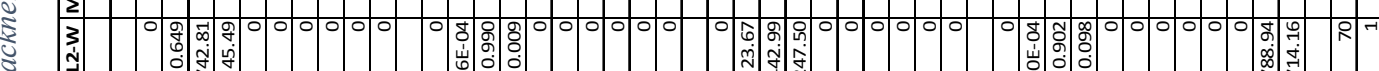

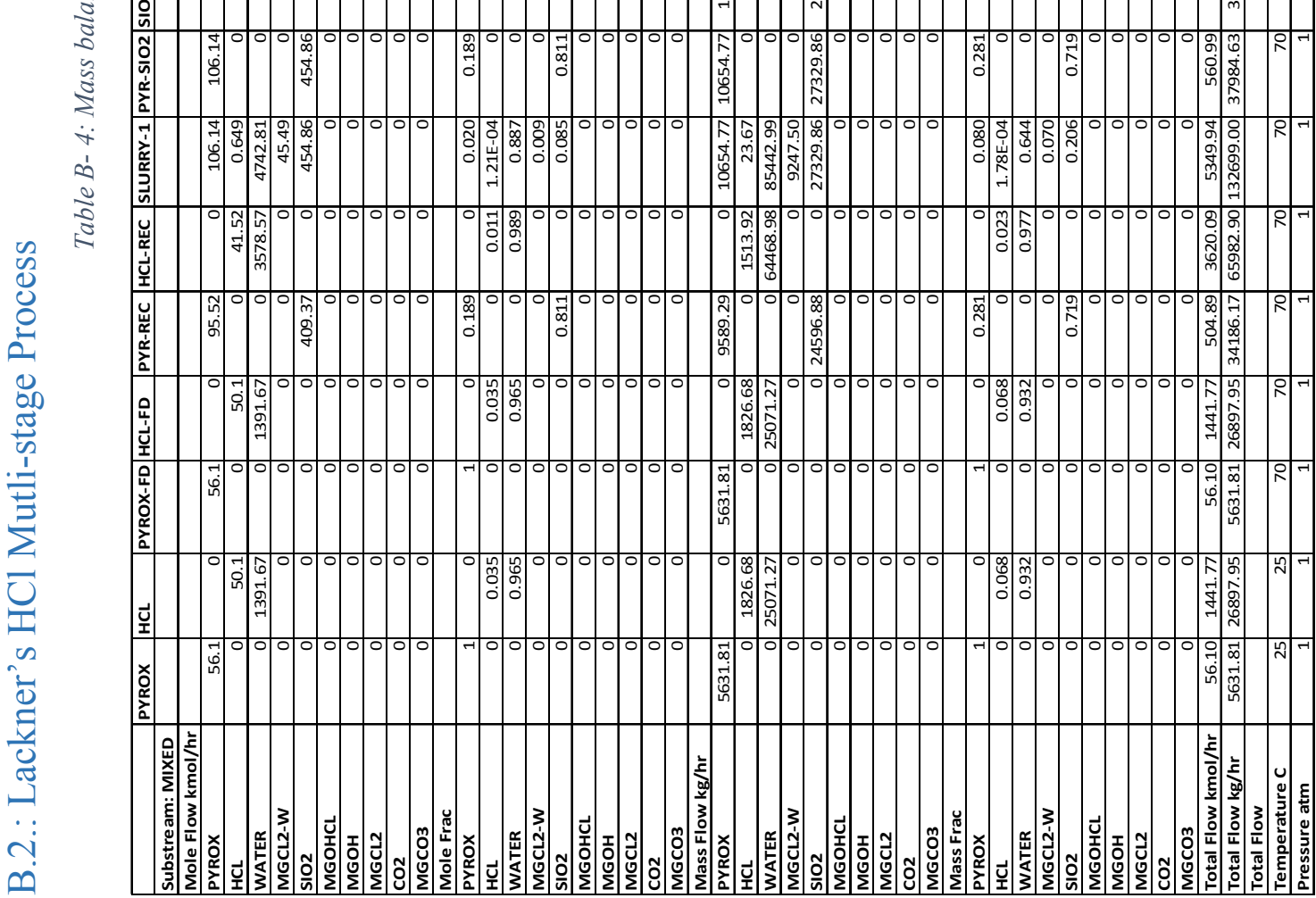




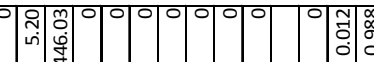

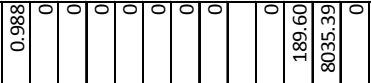

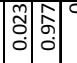

$\stackrel{\infty}{m}$

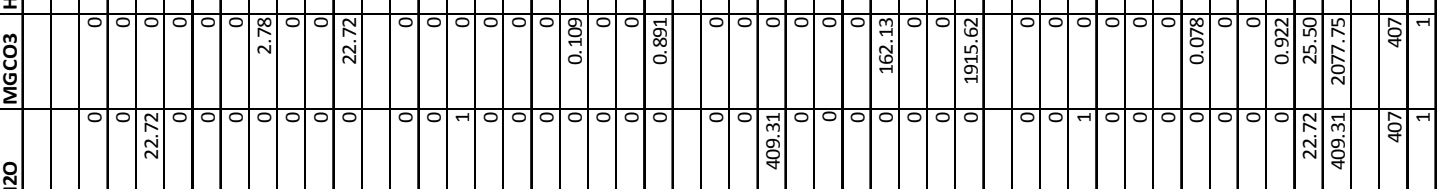

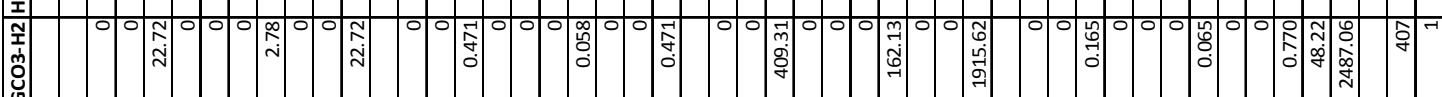

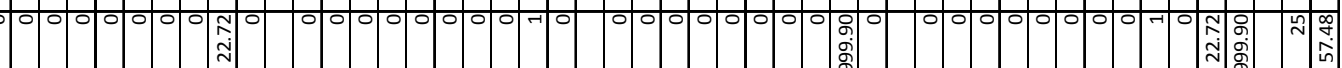

ธิ

ब

ปे

\begin{tabular}{l|l}
\hline \\
$\vdots$ \\
$\vdots$ \\
$\Sigma$
\end{tabular}

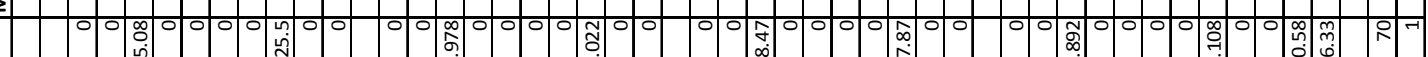

กิ่

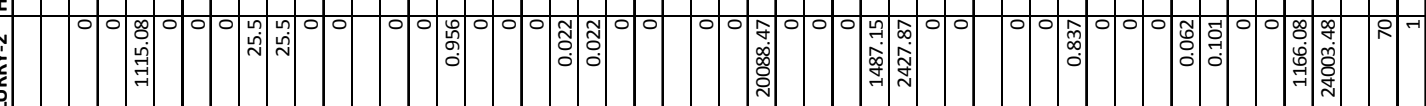

紊

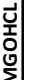

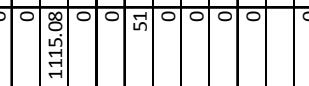

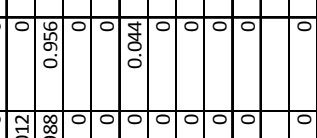



爱

产

薢

:영

萬

$\int_{0} \int_{0}$

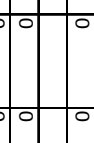

ง 0 일

ก่:

공 क्षㅇㅇ

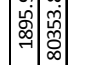

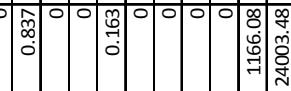

离

$\circ \circ$

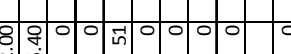

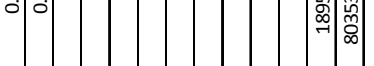

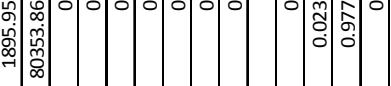

กิด

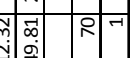

o.

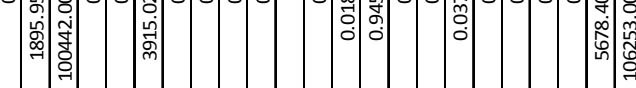

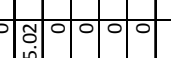

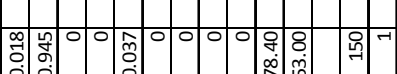

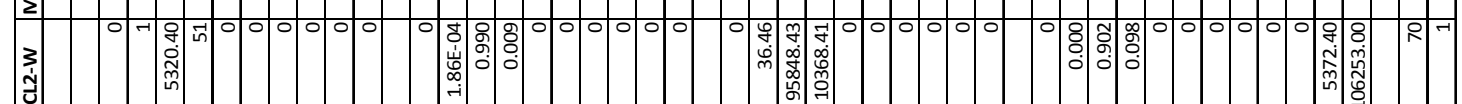

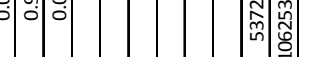

oิ

¿

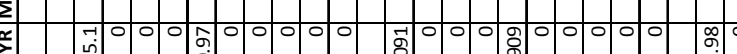

0 mo 0000

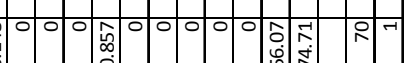

通

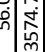

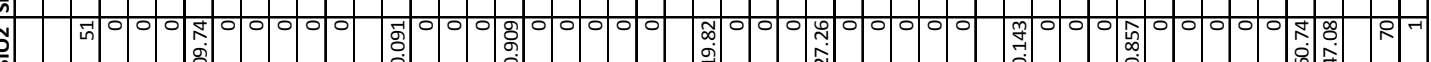

药

$\vdots$
$\dot{n}$
$\dot{a}$

$\frac{\sqrt{3}}{\frac{\pi}{3}}$

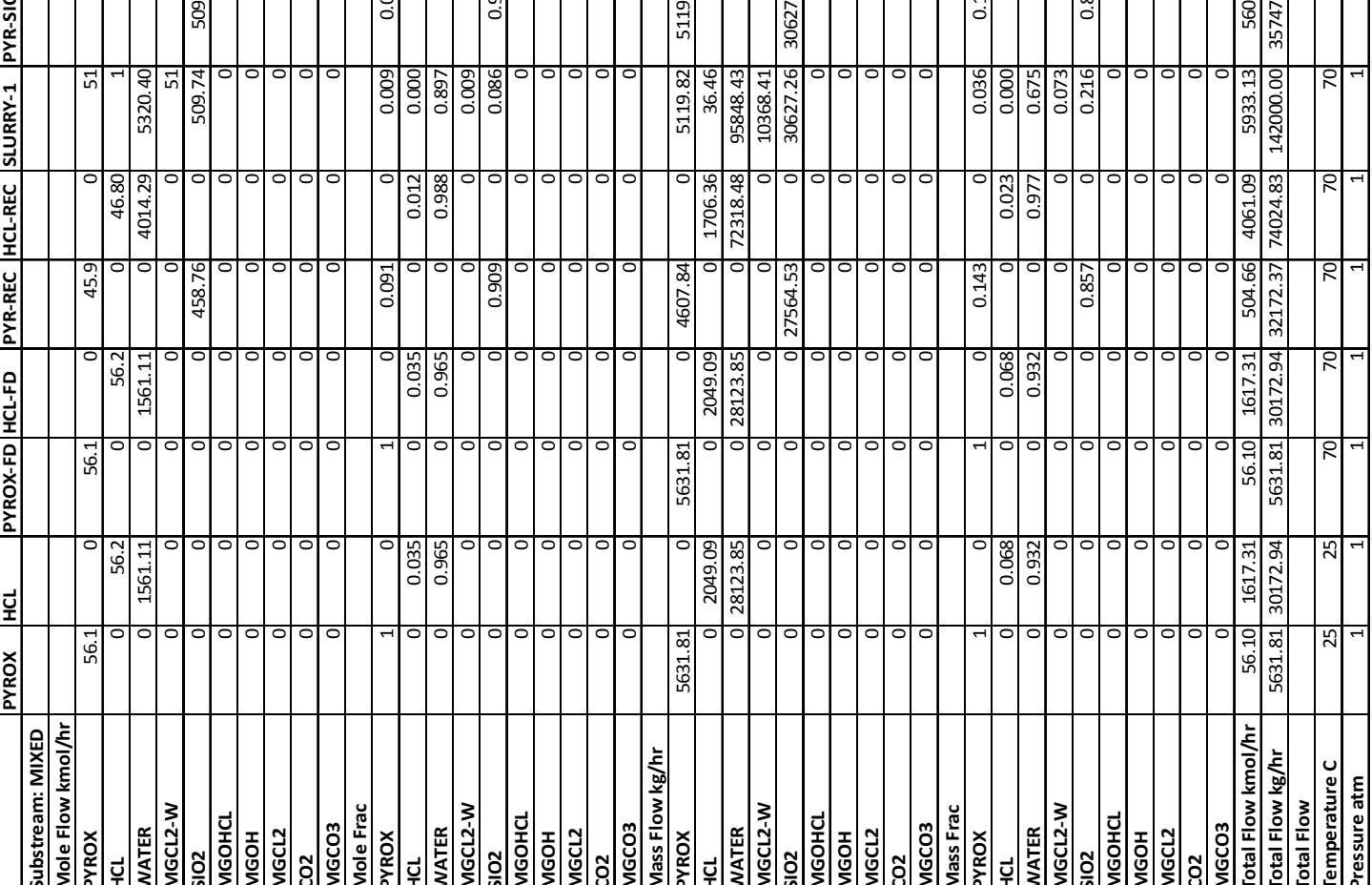




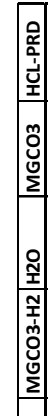

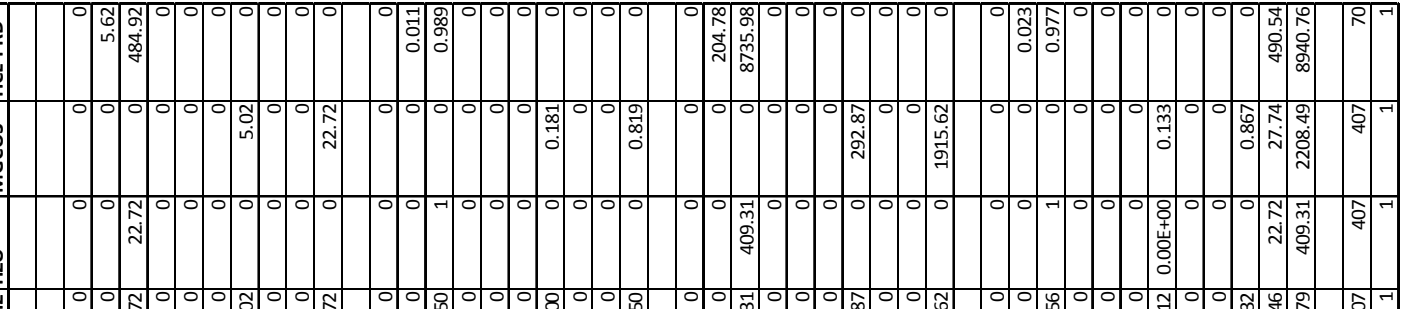

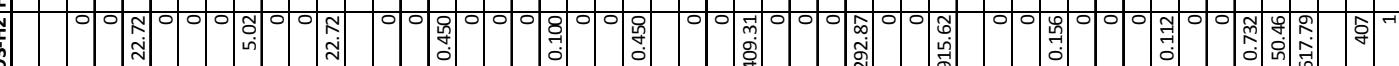

ชู

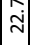
命

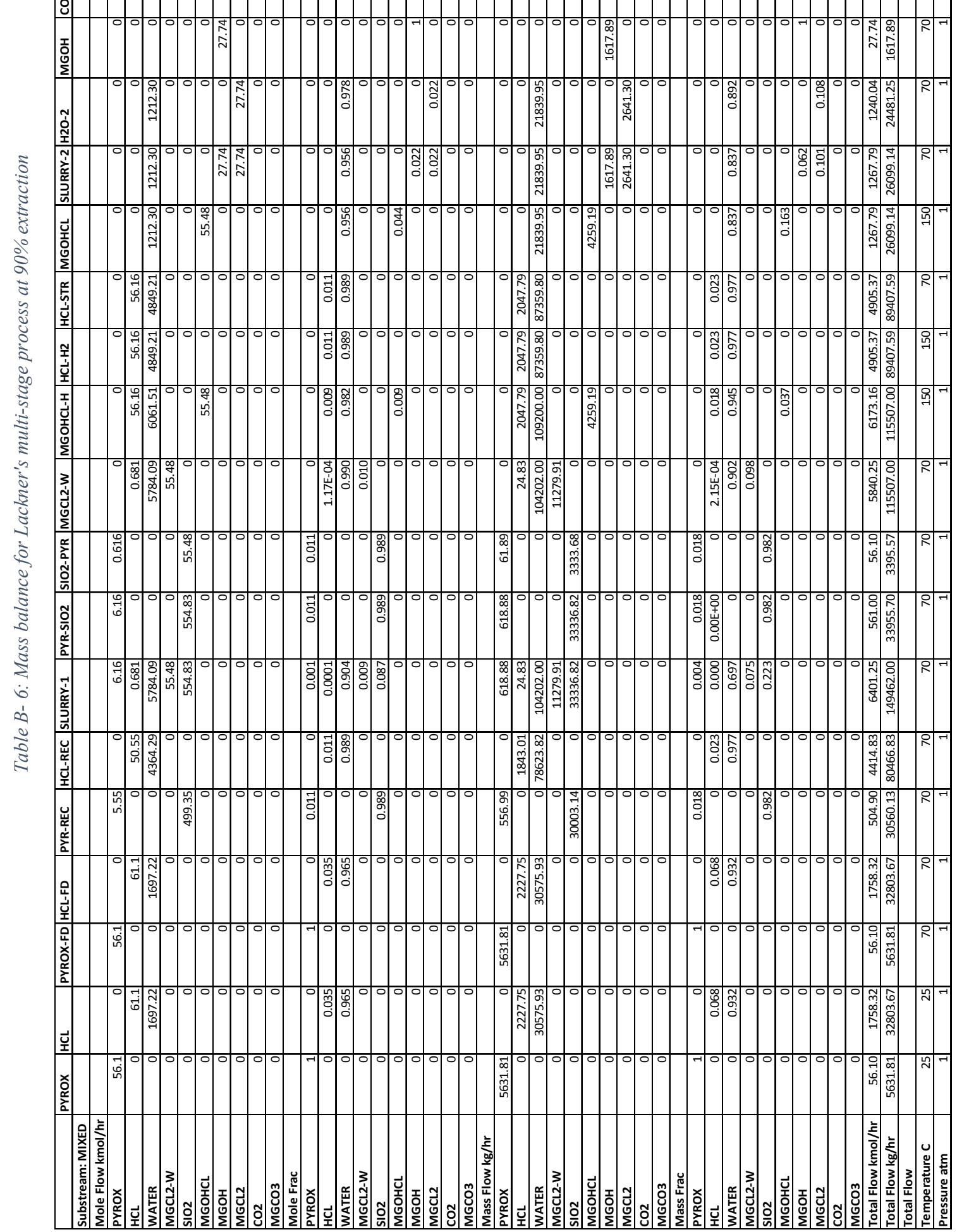



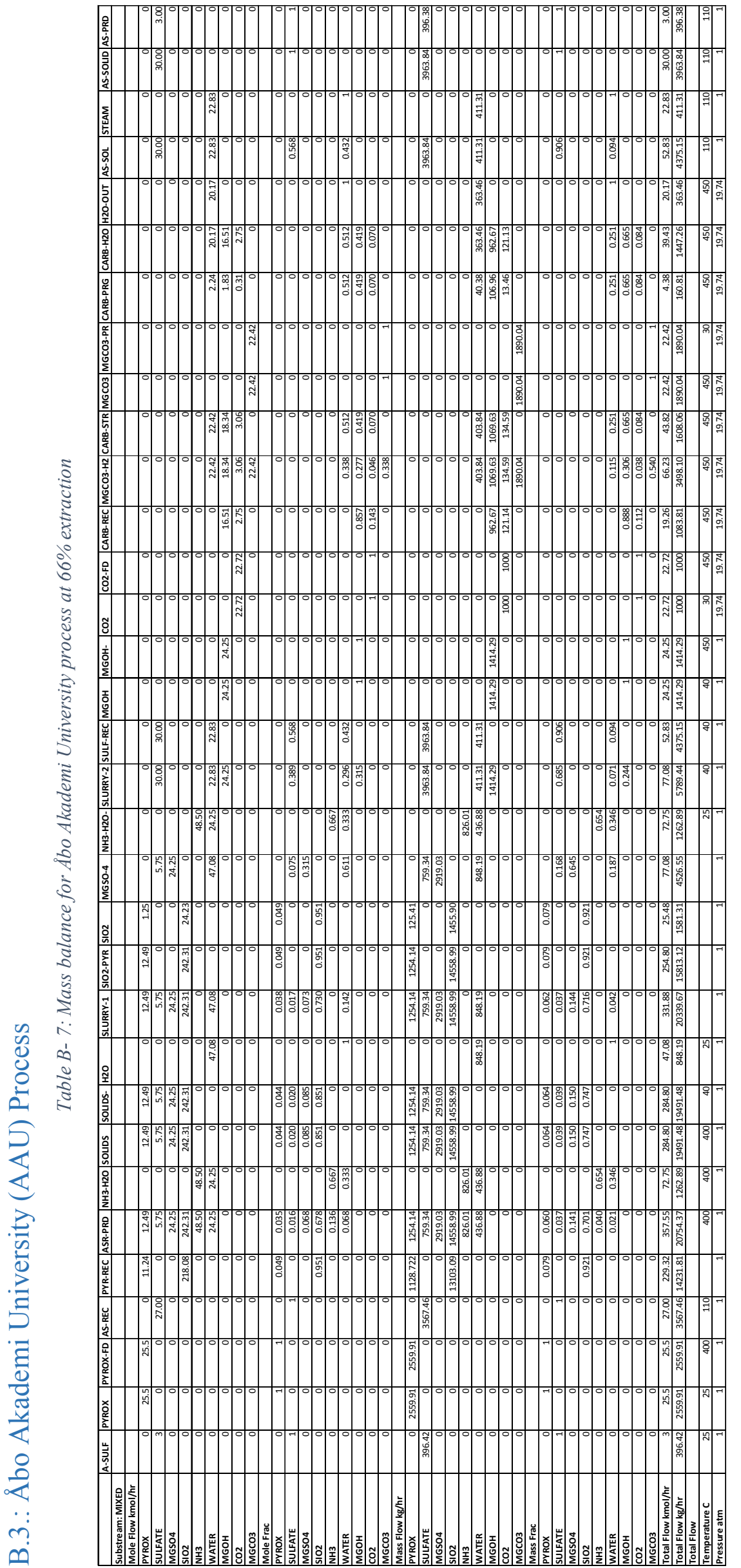


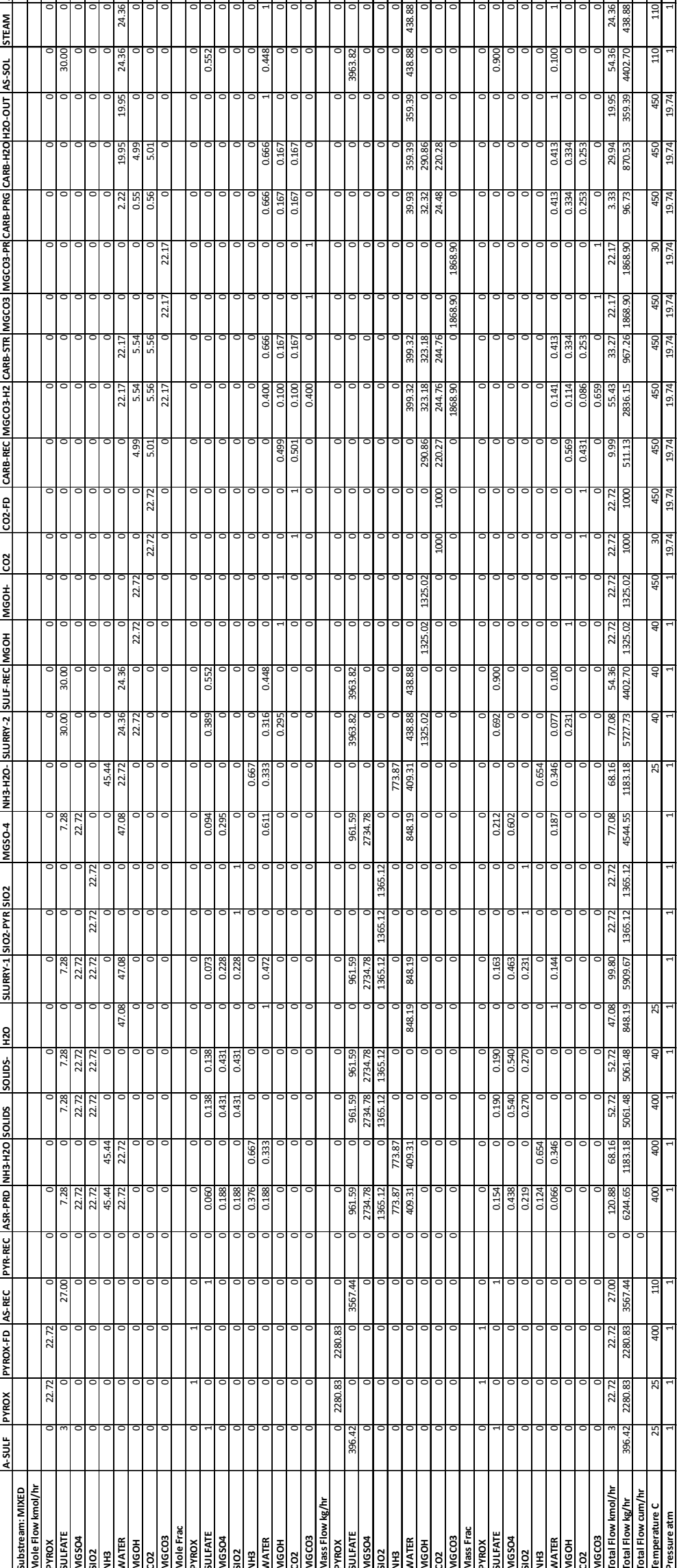



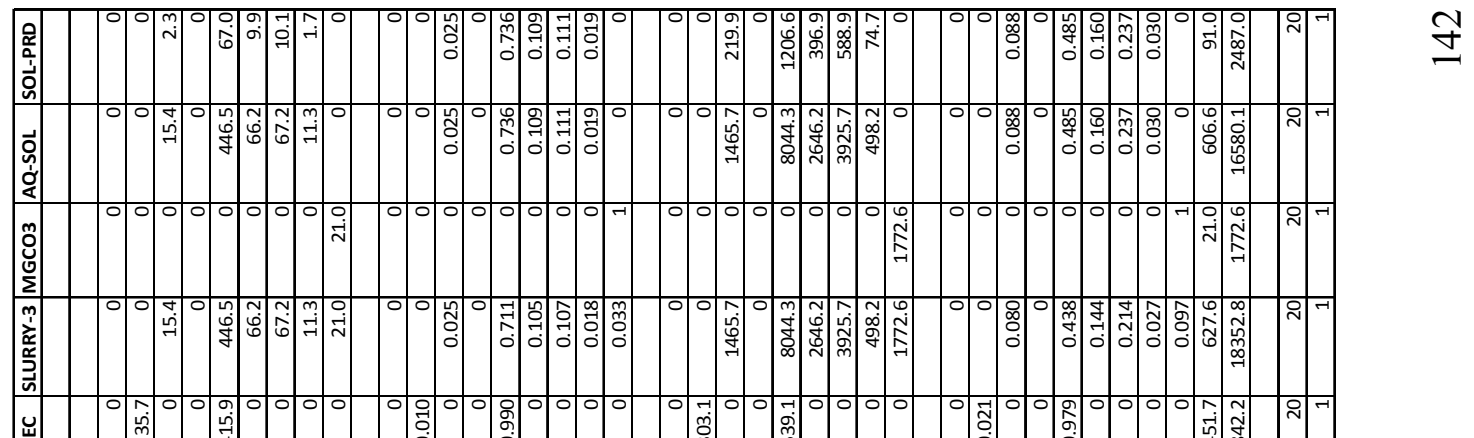

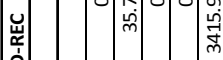

$\frac{8}{4}$

:

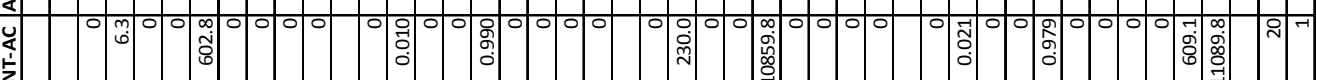

:

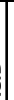

원

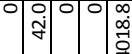

0.0000

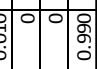

00010

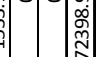

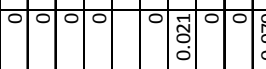

:

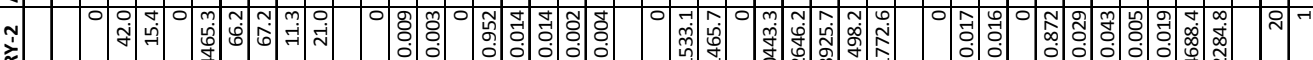

䇋

享

它

ธิ

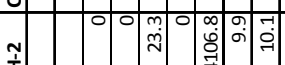

0000000

: ป

$: \sqrt{2}$

$\underline{⿶}$

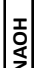

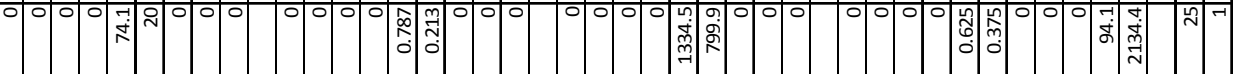

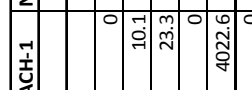

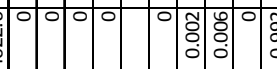

10

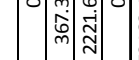

00000.

000000

辤

号

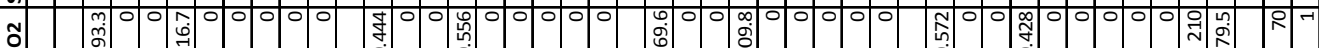

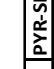

离

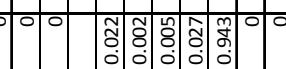

잉

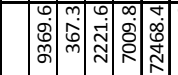

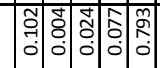

0.000 .0

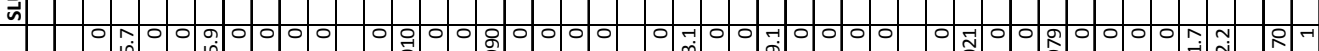

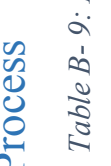

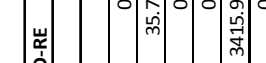

4

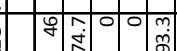

$\circ 0$

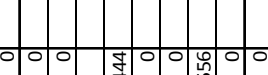

กั

0.0

-

年

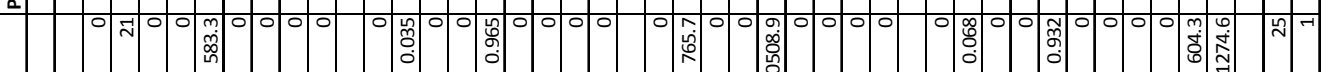

$\overrightarrow{\underline{x}}$

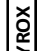




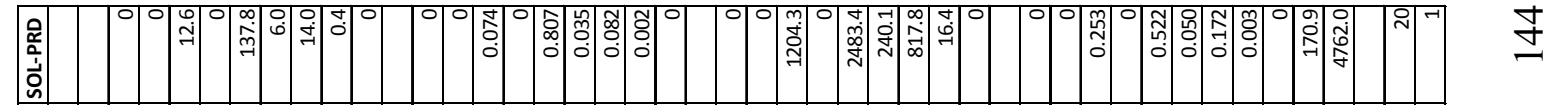

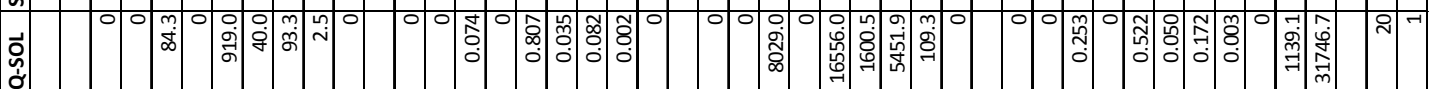

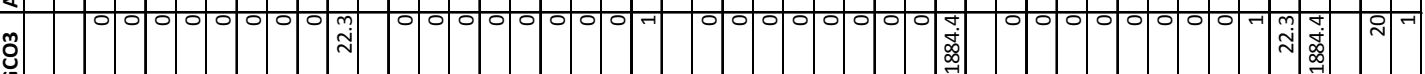

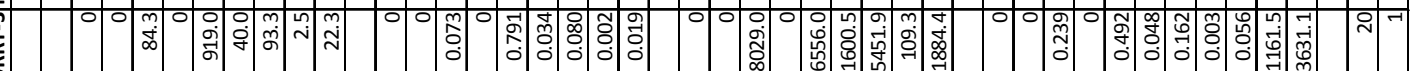

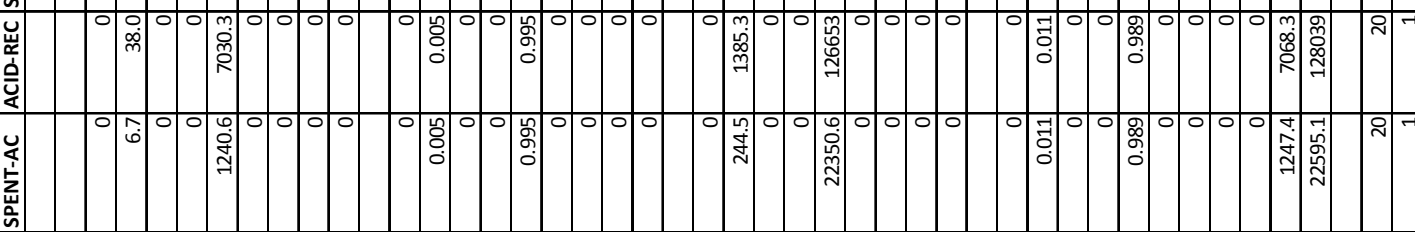
$\frac{2}{2}$

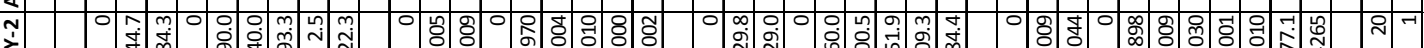
竧

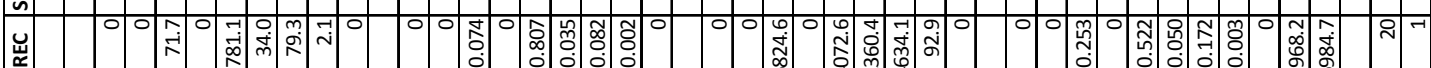
它 ปิ

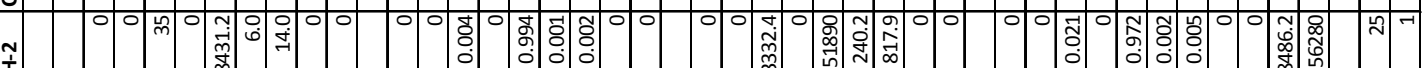
妾

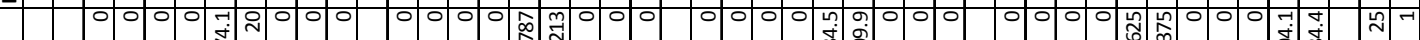

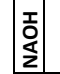

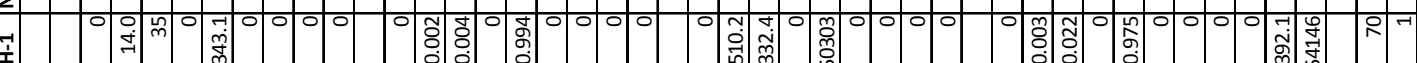



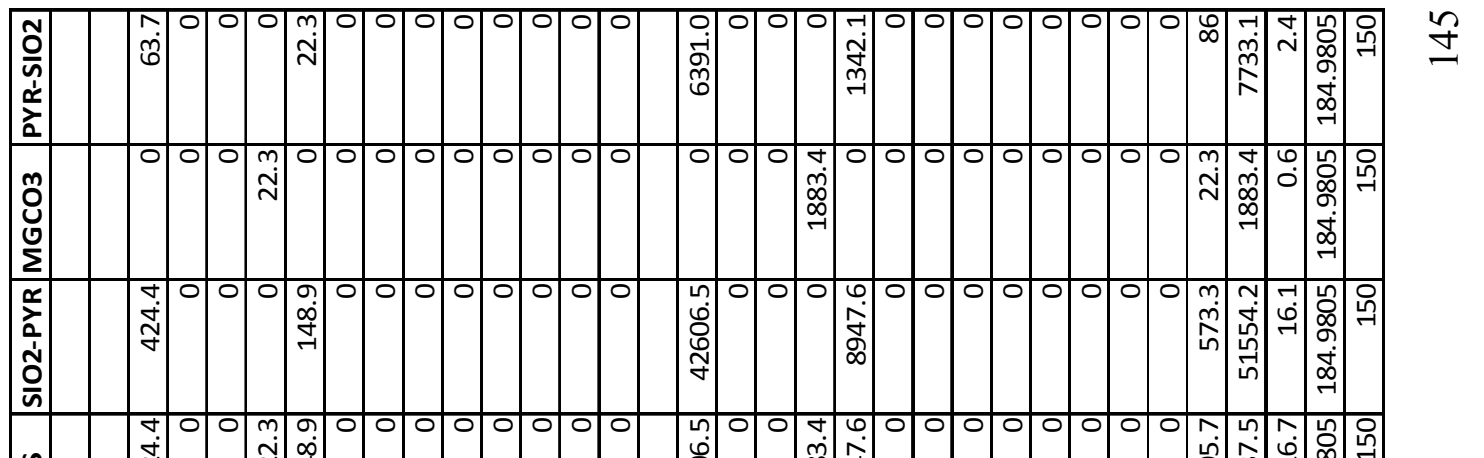

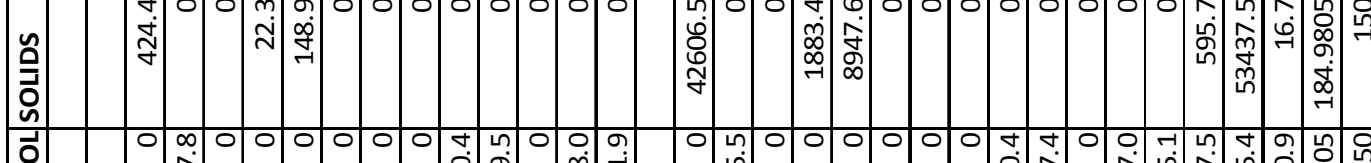

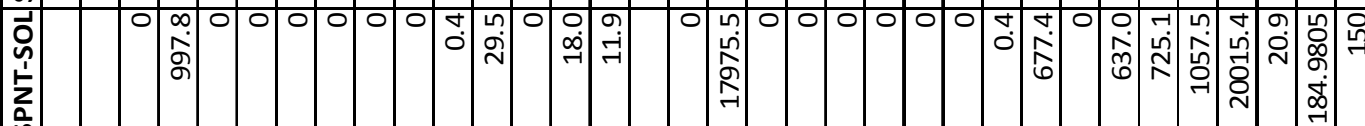

?

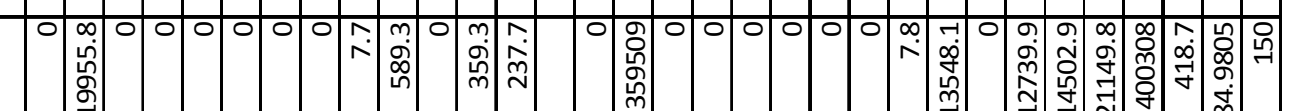
政

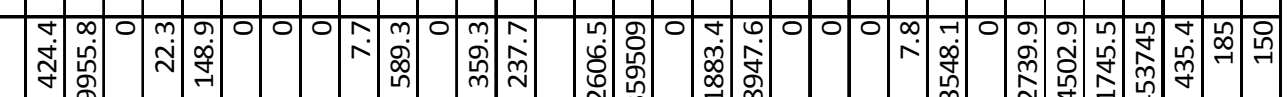

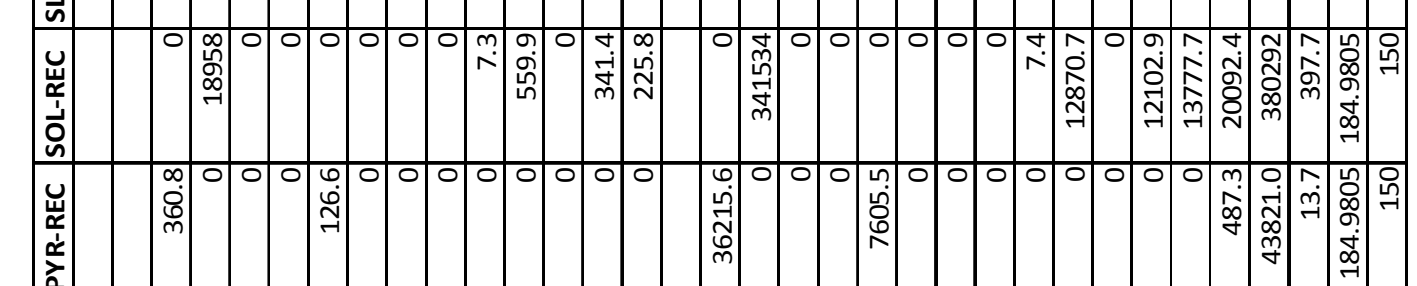
ญ

色:

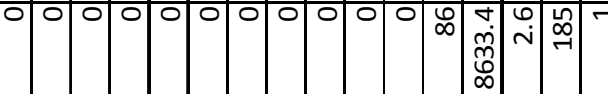

$\infty 000000000000$

I

ป்

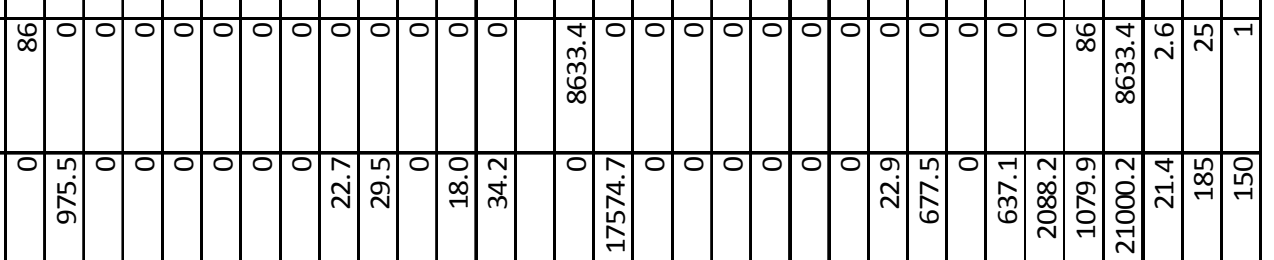

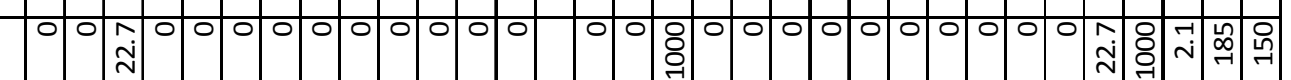

8

定

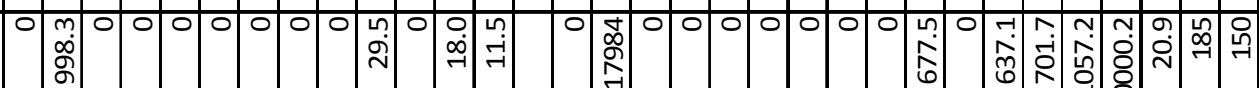

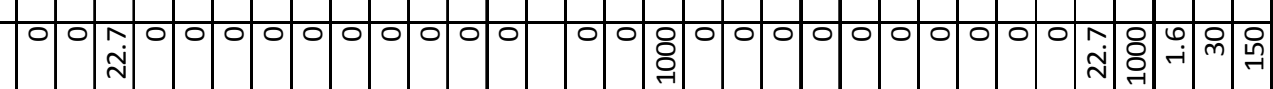

ปู่

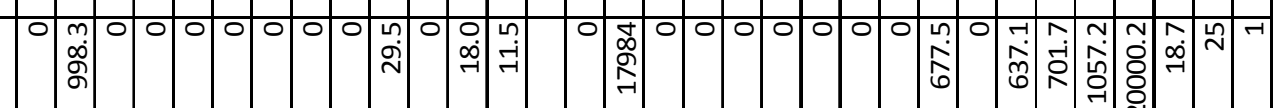

ำ

일

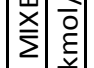

离新

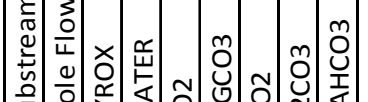

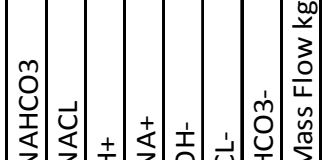

音 


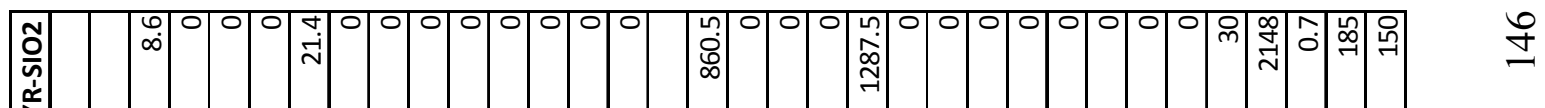

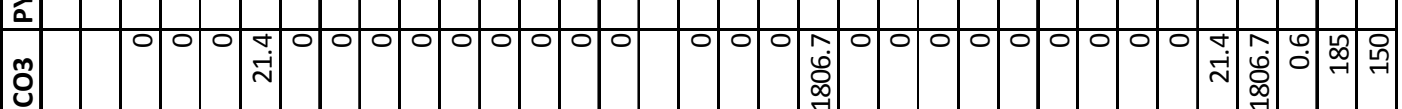

간

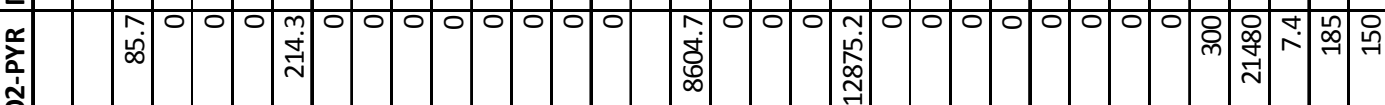

은

气.

음

它

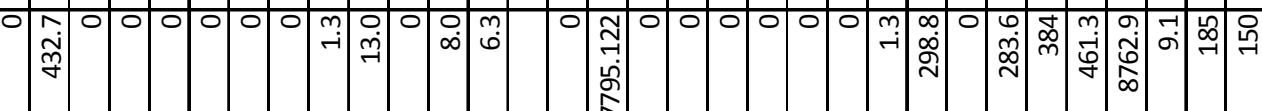

它

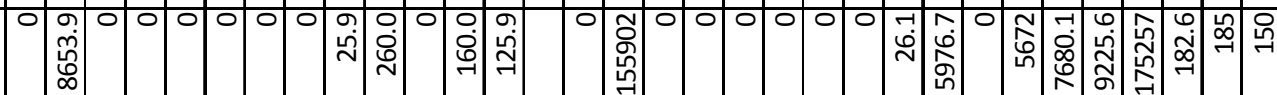

름

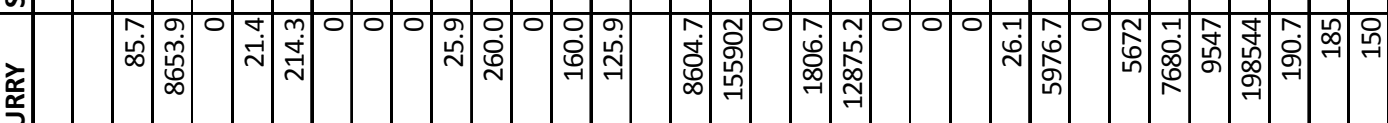

光

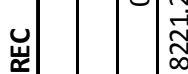



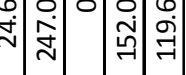

总

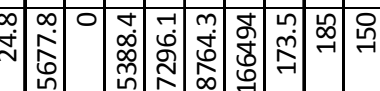

离

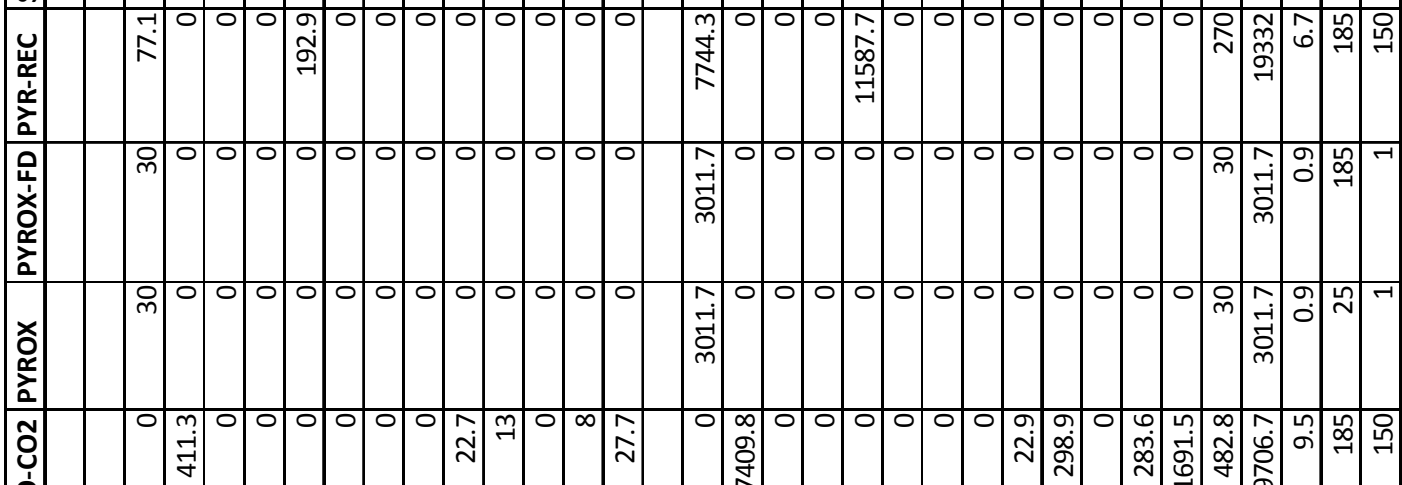

宅

ปิ่

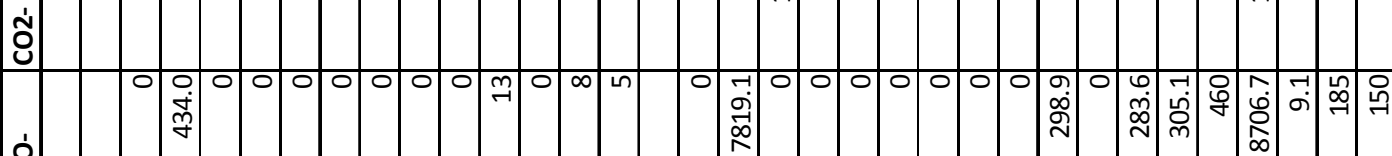

존

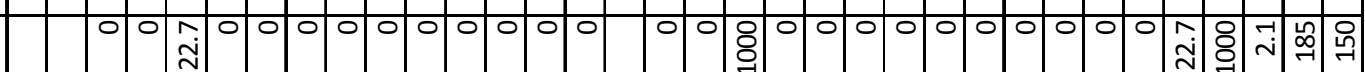

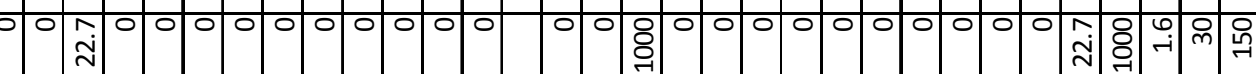

ธี

온
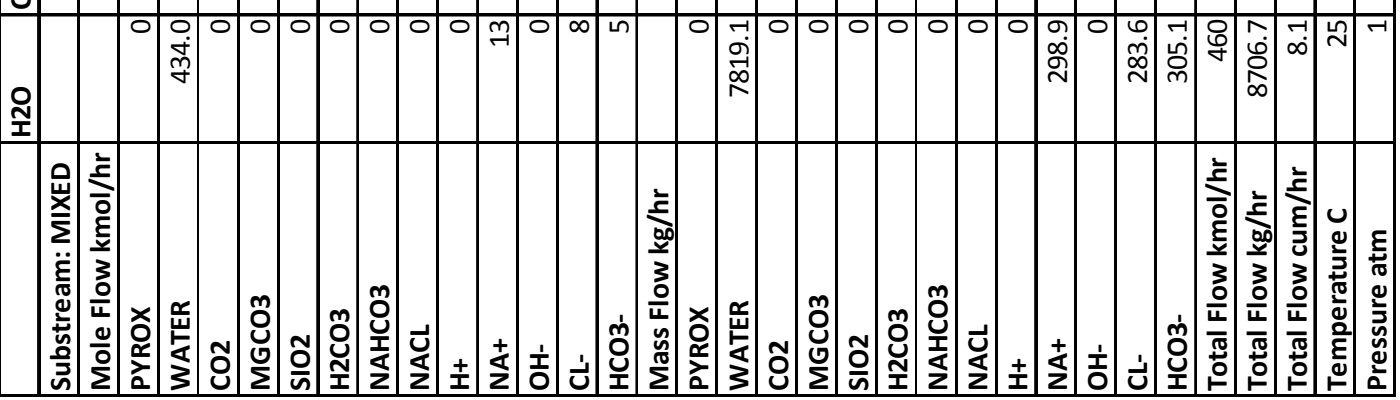


\section{Unit Energy Balances for Carbonation Processes}

\section{C.1: Ammonium Salts Process}

Table C-1: Mineral dissolution unit energy balance for 30\% extraction

\begin{tabular}{|c|c|c|c|c|c|c|c|}
\hline & PYROX & NH4HSO4 & PYROX-FD & BISUL-FD & PYR-REC & BIS-REC & SLURRY \\
\hline \multicolumn{8}{|l|}{ Substream: MIXED } \\
\hline \multicolumn{8}{|l|}{ Mole Flow kmol/hr } \\
\hline PYROX & 22.70 & 0 & 22.70 & 0 & 28.89 & 0 & 36.11 \\
\hline NH4HSO4 & 0 & 0 & 0 & 0 & 0 & 0 & 0 \\
\hline MGSO4 & 0 & 0 & 0 & 0 & 0 & 0 & 0 \\
\hline SIO2 & 0 & 0 & 0 & 0 & 61.91 & 0 & 77.39 \\
\hline NH4SO4 & 0 & 0 & 0 & 0 & 0 & 0 & 0 \\
\hline WATER & 0 & 357.14 & 0 & 357.14 & 0 & 1143.07 & 1515.69 \\
\hline $\mathrm{NH} 4 \mathrm{OH}$ & 0 & 0 & 0 & 0 & 0 & 0 & 0 \\
\hline $\mathrm{NH} 4 \mathrm{HCO} 3$ & 0 & 0 & 0 & 0 & 0 & 0 & 0 \\
\hline MGCO3 & 0 & 0 & 0 & 0 & 0 & 0 & 0 \\
\hline $\mathrm{CO2}$ & 0 & 0 & 0 & 0 & 0 & 0 & 0 \\
\hline NH3 & 0 & 0 & 0 & 0 & 0 & 0 & 0 \\
\hline MG++ & 0 & 0 & 0 & 0 & 0 & 1.35 & 16.83 \\
\hline $\mathrm{H} 3 \mathrm{O}+$ & 0 & $1.982 \mathrm{E}-06$ & 0 & $1.05 \mathrm{E}-05$ & 0 & $2.5 \mathrm{E}-10$ & $2.63 \mathrm{E}-10$ \\
\hline NH4+ & 0 & 9.00 & 0 & 9.00 & 0 & 74.17 & 83.17 \\
\hline $\mathrm{H} 2 \mathrm{SO} 4$ & 0 & $3.04 \mathrm{E}-16$ & 0 & $5.71 \mathrm{E}-13$ & 0 & $7.13 \mathrm{E}-18$ & $2.99 \mathrm{E}-18$ \\
\hline $\mathrm{OH}-$ & 0 & $1.982 \mathrm{E}-06$ & 0 & $1.05 \mathrm{E}-05$ & 0 & 9.20 & 9.20 \\
\hline HSO4- & 0 & 9.00 & 0 & 9.00 & 0 & 34.65 & 12.70 \\
\hline HCO3- & 0 & 0 & 0 & 0 & 0 & 30.32 & 30.32 \\
\hline CO3-- & 0 & 0 & 0 & 0 & 0 & 0 & 0 \\
\hline SO4-- & 0 & 0 & 0 & 0 & 0 & 1.35 & 32.30 \\
\hline Total Flow kmol/hr & 22.70 & 375.14 & 22.70 & 375.14 & 90.80 & 1294.11 & 1813.70 \\
\hline Total Flow kg/hr & 2278.82 & 7469.97 & 2278.82 & 7469.97 & 6620.05 & 27463.18 & 43832.01 \\
\hline Total Flow cum/hr & 0.681 & 7.49 & 0.681 & 7.73 & 2.27 & \begin{tabular}{|l|}
27.08 \\
\end{tabular} & 35.24 \\
\hline Temperature C & 25.00 & 25.00 & 90.00 & 90.00 & 90.00 & 90.00 & 90.00 \\
\hline Pressure atm & 1.00 & 1.00 & 1.00 & 1.00 & 1.00 & 1.00 & 1.00 \\
\hline Vapor Frac & 0 & 0 & 0 & 0 & 0 & 0 & 0 \\
\hline Liquid Frac & 0 & 1.00 & 0 & 1.00 & 0 & 1.00 & 0.937421 \\
\hline Solid Frac & 1.00 & 0 & 1.00 & 0 & 1.00 & 0 & 0.062579 \\
\hline Enthalpy kJ/kmol & -1548900 & -296720 & -1543300 & -292020 & -1109900 & -298640 & -349700 \\
\hline Enthalpy kJ/kg & -15429.2 & \begin{tabular}{|l|}
-14901.32 \\
\end{tabular} & -15373.11 & -14665.2 & \begin{tabular}{|l|}
-15222.6 \\
\end{tabular} & \begin{tabular}{|l|}
-14072.5 \\
\end{tabular} & -14469.9 \\
\hline Enthalpy kW & -9766.78 & \begin{tabular}{|l|}
-30920.1 \\
\end{tabular} & -9731.282 & -30430 & \begin{tabular}{|l|}
-27992.9 \\
\end{tabular} & \begin{tabular}{|l|}
-107350 \\
\end{tabular} & -176180 \\
\hline Entropy J/kmol-K & -291450 & -169650 & -274390 & -155380 & -205320 & -161560 & -162890 \\
\hline Entropy J/kg-K & -2903.2 & \begin{tabular}{|l|}
-8519.738 \\
\end{tabular} & \begin{tabular}{|l|}
-2733.321 \\
\end{tabular} & -7803.29 & -2816.13 & -7613.02 & -6740.2 \\
\hline Density kmol/cum & 33.33 & 50.11 & 33.33 & 48.50 & 39.98 & 47.78 & 51.47 \\
\hline Density kg/cum & 3346.29 & 997.87 & 3346.29 & 965.81 & 2914.63 & 1014.00 & 1243.98 \\
\hline Average MW & 100.39 & 19.91 & 100.39 & 19.91 & 72.91 & 21.22 & 24.17 \\
\hline Liq Vol 60F & & & & & & & \\
\hline
\end{tabular}


Table C-2: pH adjustment unit energy balance for $30 \%$ extraction

\begin{tabular}{|l|r|r|r|r|}
\hline & LEACH-1 & LEACH-2 & NH4OH & LEACH-3 \\
\hline Substream: MIXED & & & & \\
\hline Mole Flow kmol/hr & & & & \\
\hline PYROX & 0 & 0 & 0 & 0 \\
\hline NH4HSO4 & 0 & 0 & 0 & 0 \\
\hline MGSO4 & 0 & 0 & 0 & 0 \\
\hline SIO2 & 0 & 0 & 0 & 0 \\
\hline NH4SO4 & 0 & 0 & 0 & 0 \\
\hline WATER & 1515.71 & 1515.71 & 416.67 & 1945.08 \\
\hline NH4OH & 0 & 0 & 0 & 0 \\
\hline NH4HCO3 & 0 & 0 & 0 & 0 \\
\hline MGCO3 & 0 & 0 & 0 & 0 \\
\hline CO2 & 0 & 0 & 0 & 0 \\
\hline NH3 & 0 & 0 & 0 & 0 \\
\hline MG++ & 16.82 & 16.82 & 0 & 16.82 \\
\hline H3O+ & $2.63 E-10$ & $5.97 E-12$ & $1.08 E-13$ & $6.06 \mathrm{E}-12$ \\
\hline NH4+ & 83.18 & 83.18 & 15.00 & 98.18 \\
\hline H2SO4 & $2.99 E-18$ & $2.99 E-22$ & 0 & 0 \\
\hline OH- & 9.20 & 9.20 & 15.00 & 11.50 \\
\hline HSO4- & 12.70 & 12.70 & 0 & 0 \\
\hline HCO3- & 30.32 & 30.32 & 0 & 30.32 \\
\hline CO3-- & 0 & 0 & 0 & 0 \\
\hline SO4-- & 32.30 & 32.30 & 0 & 45.00 \\
\hline Total Flow kmol/hr & 1700.23 & 1700.23 & 446.67 & 2146.90 \\
\hline Total Flow kg/hr & 35557.36 & 35557.36 & 8032.11 & 43589.48 \\
\hline Total Flow cum/hr & 32.40 & 31.36 & 7.79 & 38.48 \\
\hline Temperature C & 90.00 & 25.00 & 25.00 & 25.00 \\
\hline Pressure atm & 1.00 & 1.00 & 1.00 & 1.00 \\
\hline Vapor Frac & 0 & 0 & 0 & 0 \\
\hline Liquid Frac & 1.00 & 1.00 & 1.00 & 1.00 \\
\hline Solid Frac & 0 & 0 & 0 & 0 \\
\hline Enthalpy kJ/kmol & -298950 & -303390 & -278930 & -298750 \\
\hline Enthalpy kJ/kg & -14294.7 & -14507.1 & -15511.3 & -14714.4 \\
\hline Enthalpy kW & -141190 & -143290 & -34607.9 & -178160 \\
\hline Entropy J/kmol-K & -160060 & -173530 & -165800 & -171990 \\
\hline Entropy J/kg-K & 52.48 & 54.22 & 57.37 & 55.80 \\
\hline Density kmol/cum & -8297.56 & -9220.29 & -8471.19 \\
\hline Density kg/cum & 1097.54 & 1133.97 & 1031.56 & 1132.84 \\
\hline Average MW & 20.91 & 20.91 & 17.98 & 20.30 \\
\hline
\end{tabular}




\begin{tabular}{|l|r|r|r|r|r|}
\hline & LEACH-3 & NH4-HCO3 & CARB-FD & NH4HCO3- & CARB-PRD \\
\hline Substream: MIXED & & & & & \\
\hline Mole Flow kmol/hr & & & & & 0 \\
\hline PYROX & 0 & 0 & 0 & 0 & 0 \\
\hline NH4HSO4 & 0 & 0 & 0 & 0 & 0 \\
\hline MGSO4 & 0 & 0 & 0 & 0 & 0 \\
\hline SIO2 & 0 & 0 & 0 & 0 & 0 \\
\hline NH4SO4 & 0 & 0 & 0 & 0 & 0 \\
\hline WATER & 1945.07 & 117.69 & 1945.07 & 117.69 & 2032.48 \\
\hline NH4OH & 0 & 0 & 0 & 0 & 0 \\
\hline NH4HCO3 & 0 & 0 & 0 & 0 & 0 \\
\hline MGCO3 & 0 & 0 & 0 & 0 & 15.14 \\
\hline CO2 & 0 & 0 & 0 & 0 & 15.14 \\
\hline NH3 & 16.82 & 0 & 0 & 0 & 0 \\
\hline MG++ & $0.064 \mathrm{E}-12$ & $8.5411 \mathrm{E}-07$ & $1.849 \mathrm{E}-10$ & $9.0605 \mathrm{E}-06$ & $2.2962 \mathrm{E}-10$ \\
\hline H3O+ & 98.18 & 37.86 & 98.18 & 37.86 & 136.04 \\
\hline NH4+ & 11.50 & $8.5411 \mathrm{E}-07$ & 11.50 & $9.0605 \mathrm{E}-06$ & 11.50 \\
\hline OH- & 0 & 0 & 0 & 0 & 0 \\
\hline HSO4- & 30.32 & 37.86 & 30.32 & 37.86 & 37.90 \\
\hline HCO3- & 45.00 & 0 & 45.00 & 0 & 45.00 \\
\hline SO4-- & 2146.89 & 193.41 & 2146.89 & 193.41 & 2294.89 \\
\hline Total Flow kmol/hr & 1132.84 & 1303.71 & 1103.87 & 1267.03 & 59.87 \\
\hline Total Flow kg/hr & 4358.30 & 5113.27 & 43589.30 & 5113.27 & 48702.56 \\
\hline Total Flow cum/hr & 38.48 & 3.92 & 39.49 & 4.04 & 813.44 \\
\hline Temperature C & 25.00 & 10.00 & 80.00 & 80.00 & 80.00 \\
\hline Pressure atm & 1.00 & 1.00 & 1.00 & 1.00 & 1.00 \\
\hline Vapor Frac & 0 & 0 & 0 & 0 & 0.0116367 \\
\hline Liquid Frac & 0 & 1.00 & 1.00 & 1.00 & 0.9817669 \\
\hline Solid Frac & 0 & 0 & 0 & 0.00659641 \\
\hline Enthalpy kJ/kmol & -298750 & -336060 & -294980 & -331260 & -303580 \\
\hline Enthalpy kJ/kg & -14714.36 & -12711.35 & -14528.45 & -12529.79 & -14305.03 \\
\hline Enthalpy kW & -178160 & -18054.58 & -175910 & -17796.7 & -193530 \\
\hline Entropy J/kmol-K & -171990 & -205240 & -160380 & -190110 & -165010 \\
\hline Entropy J/kg-K & -8471.188 & -7763.247 & -7899.142 & -7190.825 & -7775.24 \\
\hline Density kmol/cum & 55.80 & 49.31 & 54.37 & 47.93 & 2.82 \\
\hline Density kg/cum & 0.44 & 20.30 & 26.44 & 21.22 \\
\hline Average MW & 0 & & & \\
\hline
\end{tabular}


Table C-4: $\mathrm{CO}_{2}$ capture unit energy balance for $30 \%$ extraction

\begin{tabular}{|c|c|c|c|c|c|}
\hline & CO2-IN & $\mathrm{CO} 2-$ & CAP-H2O & CAP-NH3 & $\mathrm{NH} 4 \mathrm{HCO} 3$ \\
\hline \multicolumn{6}{|l|}{ Substream: MIXED } \\
\hline \multicolumn{6}{|l|}{ Mole Flow kmol/hr } \\
\hline PYROX & 0 & 0 & 0 & 0 & 0 \\
\hline NH4HSO4 & 0 & 0 & 0 & 0 & 0 \\
\hline MGSO4 & 0 & 0 & 0 & 0 & 0 \\
\hline S1O2 & 0 & 0 & 0 & 0 & 0 \\
\hline NH4SO4 & 0 & 0 & 0 & 0 & 0 \\
\hline WATER & 0 & 0 & 603.65 & 0 & 565.79 \\
\hline $\mathrm{NH} 4 \mathrm{OH}$ & 0 & 0 & 0 & 0 & 0 \\
\hline NH4HCO3 & 0 & 0 & 0 & 0 & 0 \\
\hline MGCO3 & 0 & 0 & 0 & 0 & 0 \\
\hline $\mathrm{CO} 2$ & 22.72 & 15.14 & 0 & 0 & 0 \\
\hline NH3 & 0 & 0 & 0 & 43.32 & 5.46 \\
\hline MG++ & 0 & 0 & 0 & 0 & 0 \\
\hline $\mathrm{H} 3 \mathrm{O}+$ & 0 & 0 & 5.896E-07 & 0 & 0.000939472 \\
\hline NH4+ & 0 & 0 & $\begin{array}{r}0 \\
\end{array}$ & 0 & 37.86 \\
\hline $\mathrm{OH}-$ & 0 & 0 & 5.896E-07 & 0 & 5.2051E-09 \\
\hline HSO4- & 0 & 0 & 0 & 0 & 0 \\
\hline HCO3- & 0 & 0 & 0 & 0 & $\begin{array}{r}37.86 \\
\end{array}$ \\
\hline CO3-- & 0 & 0 & 0 & 0 & 0.000939467 \\
\hline SO4-- & 0 & 0 & 0 & 0 & $\begin{array}{r}0 \\
\end{array}$ \\
\hline Total Flow kmol/hr & 22.72 & 15.14 & 603.65 & 43.32 & 646.97 \\
\hline Total Flow kg/hr & 999.90 & 666.31 & 10874.92 & 737.76 & 13278.90 \\
\hline Total Flow cum/hr & 524.84 & 349.74 & 10.88 & 1285.45 & 12.05 \\
\hline Temperature C & 10.00 & 10.00 & 10.00 & 90.00 & 10.00 \\
\hline Pressure atm & 1.00 & 1.00 & 1.00 & 1.00 & 1.00 \\
\hline Vapor Frac & 1.00 & 1.00 & 0 & 1.00 & 0 \\
\hline Liquid Frac & 0 & 0 & 1.00 & 0 & 1.00 \\
\hline Solid Frac & 0 & 0 & 0 & 0 & 0 \\
\hline Enthalpy kJ/kmol & -394360 & -394360 & -287140 & -43374.32 & -300050 \\
\hline Enthalpy kJ/kg & -8960.72 & -8960.723 & \begin{tabular}{|l|}
-15938.58 \\
\end{tabular} & -2546.852 & -14618.82 \\
\hline Enthalpy kW & -2488.85 & \begin{tabular}{|l|}
-1658.501 \\
\end{tabular} & \begin{tabular}{|l|}
-48147.47 \\
\end{tabular} & -521.9376 & -53922.73 \\
\hline Entropy J/kmol-K & 959.37 & 959.37 & \begin{tabular}{|l|}
-167490 \\
\end{tabular} & -91985.67 & -178360 \\
\hline Entropy J/kg-K & 21.80 & 21.80 & \begin{tabular}{|l|}
-9296.992 \\
\end{tabular} & -5401.212 & -8689.797 \\
\hline Density kmol/cum & 0.04329 & 0.0432897 & 55.50 & 0.0337003 & 53.71 \\
\hline Density kg/cum & 1.91 & 1.91 & 999.80 & 0.5739354 & 1102.40 \\
\hline Average MW & 44.01 & 44.01 & 18.02 & 17.03 & 20.52 \\
\hline
\end{tabular}


Table C-5: Thermal decomposition (EVAP) unit energy balance for 30\% extraction

\begin{tabular}{|l|r|r|}
\hline & DEC-FD & DEC-PRD \\
\hline Substream: MIXED & & \\
\hline Mole Flow kmol/hr & & \\
\hline PYROX & 0 & 0 \\
\hline NH4HSO4 & 0 & 43.32 \\
\hline MGSO4 & 0 & 0 \\
\hline SIO2 & 0 & 0 \\
\hline NH4SO4 & 43.32 & 0 \\
\hline WATER & 20.32 & 20.32 \\
\hline NH4OH & 0 & 0 \\
\hline NH4HCO3 & 0 & 0 \\
\hline MGCO3 & 0 & 0 \\
\hline CO2 & 0 & 0 \\
\hline NH3 & 0 & 43.32 \\
\hline MG++ & 1.68 & 1.68 \\
\hline H3O+ & $8.38 E-15$ & $8.34 \mathrm{E}-16$ \\
\hline NH4+ & 49.40 & 49.40 \\
\hline H2SO4 & 0 & 0 \\
\hline HSO4- & $1.1 \mathrm{E}-11$ & $3.46 \mathrm{E}-12$ \\
\hline OH- & 11.50 & 11.50 \\
\hline HCO3- & 37.90 & 37.90 \\
\hline CO3-- & 0 & 0 \\
\hline SO4-- & 1.68 & 1.68 \\
\hline Total Flow kmol/hr & 165.80 & 209.12 \\
\hline Total Flow kg/hr & 9691.86 & 9691.86 \\
\hline Total Flow kcum/hr & 0.938905 & 2.99 \\
\hline Temperature C & 300.00 & 300.00 \\
\hline Pressure atm & 1.00 & 1.00 \\
\hline Vapor Frac & 0.119859 & 0.303341 \\
\hline Liquid Frac & 0.618862 & 0.489506 \\
\hline Solid Frac & 0.261279 & 0.207154 \\
\hline Enthalpy kJ/kmol & -519090 & -388130 \\
\hline Enthalpy kJ/kg & -8880.07 & -8374.59 \\
\hline Enthalpy kW & -23906.8 & -22545.9 \\
\hline Entropy J/kmol-K & -308780 & -781040 \\
\hline Entropy J/kg-K & -5282.35 & -16852.5 \\
\hline Density kmol/cum & 0.176589 & 0.070028 \\
\hline Density kg/cum & 10.32 & 3.25 \\
\hline Average MW & 58.46 & 46.35 \\
\hline & & \\
\hline
\end{tabular}


Table C-6: Mineral carbonation unit energy balance for $90 \%$ dissolution

\begin{tabular}{|c|c|c|c|c|c|c|}
\hline & LEACH-3 & $\mathrm{NH} 4-\mathrm{HCO} 3$ & CARB-FD & NH4HCO3- & WATER & CARB-PRD \\
\hline \multicolumn{7}{|l|}{ Substream: MIXED } \\
\hline \multicolumn{7}{|l|}{ Mole Flow kmol/hr } \\
\hline PYROX & 0 & 0 & 0 & 0 & 0 & 0 \\
\hline $\mathrm{NH} 4 \mathrm{HSO} 4$ & 0 & 0 & 0 & 0 & 0 & 0 \\
\hline MGSO4 & 0 & 0 & 0 & 0 & 0 & 0 \\
\hline $\mathrm{SIO} 2$ & 0 & 0 & 0 & 0 & 0 & 0 \\
\hline $\mathrm{NH} 4 \mathrm{SO} 4$ & 0 & 0 & 0 & 0 & 0 & 0 \\
\hline WATER & 27.80 & 2.07 & 27.80 & 2.07 & 67 & 54.80 \\
\hline $\mathrm{NH} 4 \mathrm{OH}$ & 3.16 & 0 & 3.16 & 0 & 0 & 3.16 \\
\hline $\mathrm{NH} 4 \mathrm{HCO} 3$ & 0 & 0 & 0 & 0 & 0 & 0 \\
\hline MGCO3 & 0 & 0 & 0 & 0 & 0 & 21.04 \\
\hline $\mathrm{CO} 2$ & 0 & 0 & 0 & 0 & 0 & 21.04 \\
\hline $\mathrm{NH3}$ & 0.0003 & $2.50 \mathrm{E}-06$ & 0.005 & 4.07E-05 & 0 & 0.013 \\
\hline MG++ & 23.38 & 0 & 23.38 & 0 & 0 & 2.34 \\
\hline $\mathrm{H} 3 \mathrm{O}+$ & $1.32 \mathrm{E}-06$ & $2.50 \mathrm{E}-06$ & 6.31E-06 & 4.07E-05 & $1.21 \mathrm{E}-07$ & $5.45 \mathrm{E}-05$ \\
\hline $\mathrm{NH} 4+$ & 54.93 & 43.76 & 54.93 & 43.76 & 0 & 98.68 \\
\hline $\mathrm{OH}-$ & $6.38 \mathrm{E}-10$ & 7.06E-11 & 4.49E-09 & $5.11 \mathrm{E}-10$ & $1.21 \mathrm{E}-07$ & 3.49E-09 \\
\hline HSO4- & 0.0003 & 0 & 0.005 & 0 & 0 & 0.013 \\
\hline $\mathrm{HCO} 3-$ & 1.68 & 43.76 & 1.68 & 43.76 & 0 & 3.36 \\
\hline CO3-- & 0 & 0 & 0 & 0 & 0 & 0 \\
\hline SO4-- & 50.00 & 0 & 49.99 & 0 & 0 & 49.99 \\
\hline Total Flow kmol/hr & 160.96 & 89.58 & 160.96 & 89.58 & 67 & 254.43 \\
\hline Total Flow kg/hr & 7076.61 & 3496.55 & 7076.61 & 3496.55 & 1207.02 & 11780.19 \\
\hline Total Flow cum $/ \mathrm{hr}$ & 2.82 & 2.26 & 2.97 & 2.33 & 1.21 & 686.34 \\
\hline Temperature $\mathrm{C}$ & 25 & 10 & 80 & 80 & 25 & 80 \\
\hline Pressure atm & 1 & 1 & 1 & 1 & 1 & 1 \\
\hline Vapor Frac & 0 & 0 & 0 & 0 & 0 & 0.093 \\
\hline Liquid Frac & 1 & 1 & 1 & 1 & 1 & 0.825 \\
\hline Solid Frac & 0 & 0 & 0 & 0 & 0 & 0.083 \\
\hline Enthalpy $\mathrm{kJ} / \mathrm{kmol}$ & -449930 & -409070 & -448600 & -404680 & -286010 & -499490 \\
\hline Enthalpy kJ/kg & -10233.5 & -10480.47 & -10203.43 & -10368.1 & -15875.82 & -10787.96 \\
\hline Enthalpy kW & -20116.27 & -10179.31 & -20057.15 & -10070.17 & \begin{tabular}{|l|}
-5322.916 \\
\end{tabular} & -35301.16 \\
\hline Entropy J/kmol-K & -234640 & -263730 & -230310 & -249930 & -163360 & -266060 \\
\hline Entropy J/kg-K & -5336.96 & -6756.95 & -5238.33 & -6403.27 & \begin{tabular}{|l|}
-9067.981 \\
\end{tabular} & -5746.29 \\
\hline Density kmol/cum & 57.16 & 39.60 & 54.15 & 38.48 & 55.35 & 0.37 \\
\hline Density kg/cum & 2513.08 & 1545.54 & 2380.79 & 1502.04 & 997.17 & 17.16 \\
\hline Average MW & 43.97 & 39.03 & 43.97 & 39.03 & 18.02 & 46.30 \\
\hline
\end{tabular}




\section{C.2. Lackner's HCl Multi-stage Process}

Table C-7: Mineral dissolution unit energy balance for $30 \%$ extraction

\begin{tabular}{|c|c|c|c|c|c|c|c|}
\hline & PYROX & $\mathrm{HCL}$ & PYROX-FD & HCL-FD & PYR-REC & HCL-REC & SLURRY-1 \\
\hline \multicolumn{8}{|l|}{ Substream: MIXED } \\
\hline \multicolumn{8}{|l|}{ Mole Flow kmol/hr } \\
\hline PYROX & 56.1 & 0 & 56.1 & 0 & 95.52 & 0 & 106.134 \\
\hline $\mathrm{HCL}$ & 0 & 0 & 0 & 0 & 0 & 0 & 0 \\
\hline WATER & 0 & 1391.67 & 0 & 1391.67 & 0 & 3578.57 & 4742.81 \\
\hline MGCL2-W & 0 & 0 & 0 & 0 & 0 & 0 & 45.49 \\
\hline SIO2 & 0 & 0 & 0 & 0 & 409.37 & 0 & 454.86 \\
\hline MGOHCL & 0 & 0 & 0 & 0 & 0 & 0 & 0 \\
\hline MGOH & 0 & 0 & 0 & 0 & 0 & 0 & 0 \\
\hline MGCL2 & 0 & 0 & 0 & 0 & 0 & 0 & 0 \\
\hline $\mathrm{CO2}$ & 0 & 0 & 0 & 0 & 0 & 0 & 0 \\
\hline MGCO3 & 0 & 0 & 0 & 0 & 0 & 0 & 0 \\
\hline MG++ & 0 & 0 & 0 & 0 & 0 & 0 & 0 \\
\hline $\mathrm{H}+$ & 0 & 50.1 & 0 & 50.1 & 0 & 41.52 & 0.648 \\
\hline OH- & 0 & $3.29 \mathrm{E}-13$ & 0 & $4.40 \mathrm{E}-12$ & 0 & $4.31 \mathrm{E}-11$ & 2.17E-09 \\
\hline HCO3- & 0 & 0 & 0 & 0 & 0 & 0 & 0 \\
\hline CL- & 0 & 50.1 & 0 & 50.1 & 0 & 41.52 & 0.648 \\
\hline CO3-- & 0 & 0 & 0 & 0 & 0 & 0 & 0 \\
\hline \multicolumn{8}{|l|}{ Mole Frac } \\
\hline PYROX & 1 & 0 & 1 & 0 & 0.189 & 0 & 0.020 \\
\hline $\mathrm{HCL}$ & 0 & 0 & 0 & 0 & 0 & 0 & 0 \\
\hline WATER & 0 & 0.933 & 0 & 0.933 & 0 & 0.977 & 0.886 \\
\hline MGCL2-W & 0 & 0 & 0 & 0 & 0 & 0 & 0.009 \\
\hline S1O2 & 0 & 0 & 0 & 0 & 0.811 & 0 & 0.085 \\
\hline MGOHCL & 0 & 0 & 0 & 0 & 0 & 0 & 0 \\
\hline MGOH & 0 & 0 & 0 & 0 & 0 & 0 & 0 \\
\hline MGCL2 & 0 & 0 & 0 & 0 & 0 & 0 & 0 \\
\hline $\mathrm{CO2}$ & 0 & 0 & 0 & 0 & 0 & 0 & 0 \\
\hline MGCO3 & 0 & 0 & 0 & 0 & 0 & 0 & 0 \\
\hline MG++ & 0 & 0 & 0 & 0 & 0 & 0 & 0 \\
\hline $\mathrm{H}+$ & 0 & 0.034 & 0 & 0.034 & 0 & 0.011 & 0.000 \\
\hline $\mathrm{OH}-$ & 0 & $2.20 \mathrm{E}-16$ & 0 & $2.95 \mathrm{E}-15$ & 0 & $1.18 \mathrm{E}-14$ & $4.06 \mathrm{E}-13$ \\
\hline HCO3- & 0 & 0 & 0 & 0 & 0 & 0 & 0 \\
\hline CL- & 0 & 0.034 & 0 & 0.034 & 0 & 0.011 & 0.000 \\
\hline CO3-- & 0 & 0 & 0 & 0 & 0 & 0 & 0 \\
\hline Total Flow kmol/hr & 56.1 & 1491.87 & 56.1 & 1491.87 & 504.89 & 3661.61 & 5350.58 \\
\hline Total Flow kg/hr & 5631.81 & 26898 & 5631.81 & 26898 & 34185.84 & 65982.79 & 132698 \\
\hline Total Flow cum/hr & 1.68 & 26.98 & 1.683 & 27.52 & 12.15 & 67.46 & 104.52 \\
\hline Temperature C & 25 & 25 & 70 & 70 & 70 & 70 & 70 \\
\hline Pressure atm & 1 & 1 & 1 & 1 & 1 & 1 & 1 \\
\hline Vapor Frac & 0 & 0 & 0 & 0 & 0 & 0 & 0 \\
\hline Liquid Frac & 0 & 1 & 0 & 1 & 0 & 1 & 0.887 \\
\hline Solid Frac & 1 & 0 & 1 & 0 & 1 & 0 & 0.113 \\
\hline Enthalpy kJ/kmol & -1548900 & -272500 & -1545100 & -269440 & -1029000 & -278170 & -379550 \\
\hline Enthalpy kJ/kg & -15429.2 & -15113.86 & -15390.95 & -14944.09 & -15197.37 & -15436.42 & -15303.84 \\
\hline Enthalpy kW & -24137.29 & -112930 & -24077.46 & -111660 & -144320 & -282930 & -564110 \\
\hline Entropy J/kmol-K & -291450 & -156090 & -279470 & -146530 & -195500 & -150330 & -166330 \\
\hline Entropy J/kg-K & -2903.20 & -8657.55 & -2783.83 & -8127.22 & -2887.37 & -8342.58 & -6706.67 \\
\hline Density kmol/cum & 33.33 & 55.30 & 33.33 & 54.22 & 41.54 & 54.28 & 51.19 \\
\hline Density kg/cum & 3346.29 & 997.01 & 3346.29 & 977.54 & 2812.87 & 978.14 & 1269.62 \\
\hline Average MW & 100.39 & 18.03 & 100.39 & 18.03 & 67.71 & 18.02 & 24.80 \\
\hline
\end{tabular}


Table C-8: Conversion and repartition unit energy balance for $30 \%$ extraction

\begin{tabular}{|c|c|c|c|c|}
\hline & \multicolumn{2}{|c|}{ Conversion } & \multicolumn{2}{|c|}{ Repartition } \\
\hline & MGCL2-W & MGOHCL-H & MGOHCL & SLURRY-2 \\
\hline \multicolumn{5}{|l|}{ Substream: MIXED } \\
\hline Mole Flow kmol/hr & & & 0 & 0 \\
\hline MGSIO3 & 0 & 0 & 0 & 0 \\
\hline $\mathrm{HCL}$ & 0 & 0 & 994.05 & 994.05 \\
\hline WATER & 4742.81 & 4970.26 & 0 & 0 \\
\hline MGCL2-W & 45.49 & 0 & 0 & $\underline{0}$ \\
\hline $\mathrm{SIO} 2$ & 0 & 0 & 45.49 & 0 \\
\hline $\mathrm{MGOHCL}$ & 0 & 45.49 & 0 & 22.75 \\
\hline $\mathrm{MGOH}$ & 0 & 0 & 0 & 0 \\
\hline MGCL2 & 0 & 0 & 0 & $\underline{0}$ \\
\hline $\mathrm{CO} 2$ & 0 & 0 & 0 & $\underline{0}$ \\
\hline MGCO3 & 0 & 0 & 0 & 22.75 \\
\hline MG++ & 0 & 0 & $7.12 \mathrm{E}-06$ & $4.78 \mathrm{E}-06$ \\
\hline $\mathrm{H}+$ & 0.649 & 46.139 & 7.12E-06 & 4.78E-06 \\
\hline $\mathrm{OH}-$ & 2.17E-09 & $6.70 \mathrm{E}-15$ & 0 & 45.49 \\
\hline $\mathrm{CL}-$ & 0.649 & 46.14 & 1039.54 & 1085.03 \\
\hline Total Flow kmol/hr & 4789.60 & 5108.03 & 21400.13 & 21400.13 \\
\hline Total Flow kg/hr & 94714.92 & 94714.92 & 19.68 & 19.27 \\
\hline Total Flow cum $/ \mathrm{hr}$ & 91.01 & 167803 & 70 & 70 \\
\hline Temperature $\mathrm{C}$ & 70 & 150 & 1 & 1 \\
\hline Pressure atm & 1 & 1 & 0 & 0 \\
\hline Vapor Frac & 0 & 0.951 & 0.956 & 0.979 \\
\hline Liquid Frac & 0.991 & 0.040 & 0.044 & 0.021 \\
\hline Solid Frac & 0.009 & 0.009 & -305090 & -295280 \\
\hline Enthalpy kJ/kmol & -303480 & -240540 & -14820.2 & -14971.27 \\
\hline Enthalpy kJ/kg & -15346.51 & -12972.19 & -88098.38 & -88996.45 \\
\hline Enthalpy kW & -403760 & -341290 & -155430 & -151770 \\
\hline Entropy J/kmol-K & -162910 & -37015.43 & -7550.30 & -7694.89 \\
\hline Entropy J/kg-K & -8238.35 & -1996.26 & 52.82 & 56.30 \\
\hline Density kmol/cum & 52.62 & 0.030 & 1087.34 & 1110.34 \\
\hline Density kg/cum & 1040.65 & 0.564 & 20.59 & 19.72 \\
\hline Average MW & 19.78 & 18.54 & & \\
\hline Liq Vol 60F & & & & \\
\hline
\end{tabular}


Table C-9: Mineral carbonation unit energy balance for $30 \%$ extraction

\begin{tabular}{|l|r|r|r|}
\hline & \multicolumn{1}{|l|}{ CO2 } & MGOH & MGOH-H2 \\
\hline Substream: MIXED & & & \\
\hline Mole Flow kmol/hr & & & 0 \\
\hline MGSIO3 & 0 & 0 & 0 \\
\hline HCL & 0 & 0 & 0 \\
\hline WATER & 0 & 0 & 22.72 \\
\hline MGCL2-W & 0 & 0 & 0 \\
\hline SIO2 & 0 & 0 & 0 \\
\hline MGOHCL & 0 & 0 & 0 \\
\hline MGOH & 0 & 22.74 & 0.02 \\
\hline MGCL2 & 0 & 0 & 0 \\
\hline CO2 & 22.72 & 0 & 0 \\
\hline MGCO3 & 0 & 0 & 22.72 \\
\hline Total Flow kmol/hr & 22.72 & 22.74 & 45.46 \\
\hline Total Flow kg/hr & 999.90 & 1326.19 & 2326.09 \\
\hline Total Flow cum/hr & 5.84 & 0.560 & 1266.65 \\
\hline Temperature C & 25 & 70 & 406.85 \\
\hline Pressure atm & 57.48 & 1 & 1 \\
\hline Vapor Frac & 1 & 0 & 0.500 \\
\hline Liquid Frac & 0 & 0 & 0 \\
\hline Solid Frac & 0 & 1 & 0.500 \\
\hline Enthalpy kJ/kmol & -396560 & -920870 & -652300 \\
\hline Enthalpy kJ/kg & -9010.61 & -15790.09 & -12748.2 \\
\hline Enthalpy kW & -2502.704 & -5816.85 & -8237.08 \\
\hline Entropy J/kmol-K & -38314.09 & -293890 & -109110 \\
\hline Entropy J/kg-K & -870.58 & -5039.27 & -2132.35 \\
\hline Density kmol/cum & 3.89 & 40.60 & 0.036 \\
\hline Density kg/cum & 171.07 & 2367.83 & 1.84 \\
\hline Average MW & 44.01 & 58.32 & 51.17 \\
\hline Liq Vol 60F cum/hr & 1.22 & & \\
\hline
\end{tabular}




\section{C.3. Åbo Akademi University (AAU) Process}

Table C-10: Mg-extraction (AS-REAC) unit energy balance for $66 \%$ extraction and 55\% carbonation

\begin{tabular}{|l|r|r|r|r|r|r|}
\hline & A-SULF & PYROX & PYROX-FD & PYR-REC & AS-REC & ASR-PRD \\
\hline Substream: MIXED & & & & & & \\
\hline Mole Flow kmol/hr & & & & & & \\
\hline PYROX & 0 & 25.5 & 25.5 & 11.24 & 0 & 12.49 \\
\hline SULFATE & 3 & 0 & 0 & 0 & 27.00 & 5.75 \\
\hline MGSO4 & 0 & 0 & 0 & 0 & 0 & 24.25 \\
\hline SIO2 & 0 & 0 & 0 & 218.08 & 0 & 242.33 \\
\hline NH3 & 0 & 0 & 0 & 0 & 0 & 48.4968 \\
\hline WATER & 0 & 0 & 0 & 0 & 0 & 24.25 \\
\hline MGOH & 0 & 0 & 0 & 0 & 0 & 0 \\
\hline CO2 & 0 & 0 & 0 & 0 & 0 & 0 \\
\hline MGCO3 & 0 & 0 & 0 & 0 & 0 & 0 \\
\hline Total Flow kmol/hr & 3 & 25.5 & 25.5 & 229.32 & 27.00 & 357.57 \\
\hline Total Flow kg/hr & 396.42 & 2559.91 & 2559.91 & 14231.55 & 3567.796 & 20755.68 \\
\hline Total Flow cum/hr & 0.224 & 0.765 & 0.765 & 5.29 & 2.03 & 4025.60 \\
\hline Temperature C & 25 & 25 & 400 & 40 & 110 & 400 \\
\hline Pressure atm & 1 & 1 & 1 & 1 & 1 & 1 \\
\hline Vapor Frac & 0 & 0 & 0 & 0 & 0 & 0.203 \\
\hline Liquid Frac & 0 & 0 & 0 & 0 & 0 & 0 \\
\hline Solid Frac & 1 & 1 & 1 & 1 & 1 & 0.797 \\
\hline Enthalpy kJ/kmol & -1180900 & -1548900 & -1511700 & -941270 & -1163900 & -776830 \\
\hline Enthalpy kJ/kg & -8936.315 & -15429.2 & -15058.18 & -15167.2 & -8807.85 & -13382.7 \\
\hline Enthalpy MJ/hr & -3542.55 & -39497.38 & -38547.61 & -215850 & -31424.6 & -277770 \\
\hline Entropy J/kmol-K & -936350 & -291450 & -212070 & -185520 & -886380 & -407610 \\
\hline Entropy J/kg-K & -7086.02 & -2903.204 & -2112.46 & -2989.32 & -6707.86 & -7021.98 \\
\hline Density kmol/cum & 13.41 & 33.33 & 33.33 & 43.37 & 13.29 & 0.089 \\
\hline Density kg/cum & 1772.402 & 3346.29 & 3346.29 & 2691.47 & 1755.94 & 5.16 \\
\hline Average MW & 132.14 & 100.39 & 100.39 & 62.06 & 132.14 & 58.05 \\
\hline Liq Vol 60F & & & & & & \\
\hline
\end{tabular}




\begin{tabular}{|l|r|r|r|r|}
\hline & NH3-H2O & NH3-H2O- & MGSO-4 & SLURRY-2 \\
\hline Substream: MIXED & & & & \\
\hline Mole Flow kmol/hr & & & & \\
\hline PYROX & 0 & 0 & 0 & 0 \\
\hline SULFATE & 0 & 0 & 0 & 0 \\
\hline MGSO4 & 0 & 0 & 0 & 0 \\
\hline SIO2 & 0 & 0 & 0 & 0 \\
\hline NH3 & 48.5 & 48.5 & 0 & 0 \\
\hline WATER & 24.25 & 24.25 & 47.08 & 22.83 \\
\hline MGOH & 0 & 0 & 0 & 24.25 \\
\hline CO2 & 0 & 0 & 0 & 0 \\
\hline MGCO3 & 0 & 0 & 0 & 0 \\
\hline MG++ & 0 & 0 & 24.25 & 0 \\
\hline H3O+ & 0 & $1.34 \mathrm{E}-09$ & $1.42 \mathrm{E}-07$ & $7.26 \mathrm{E}-08$ \\
\hline NH4+ & 0 & 0 & 11.50 & 60.00 \\
\hline HCO3- & 0 & 0 & 0 & 0 \\
\hline OH- & 0 & $1.34 \mathrm{E}-09$ & $1.42 \mathrm{E}-07$ & $7.26 \mathrm{E}-08$ \\
\hline CO3-- & 0 & 0 & 0 & 0 \\
\hline SO4-- & 0 & 0 & 30.00 & 30.00 \\
\hline Total Flow kmol/hr & 72.75 & 72.75 & 112.83 & 137.08 \\
\hline Total Flow kg/hr & 1262.85 & 1262.85 & 4526.906 & 5789.76 \\
\hline Total Flow cum/hr & 4014.98 & 957.35 & 1.11 & 3.19 \\
\hline Temperature C & 400 & 25 & 40 & 40 \\
\hline Pressure atm & 1 & 1 & 1 & 1 \\
\hline Vapor Frac & 1 & 0.541 & 0 & 0 \\
\hline Liquid Frac & 0 & 0.459 & 1 & 0.823 \\
\hline Solid Frac & 0 & 0 & 0 & 0.177 \\
\hline Enthalpy kJ/kmol & -96373.39 & -129260 & -471870 & -466030 \\
\hline Enthalpy kJ/kg & -5551.85 & -7446.08 & -11761.14 & -11033.88 \\
\hline Enthalpy MJ/hr & -7011.164 & -9403.30 & -53241.57 & -63883.49 \\
\hline Entropy J/kmol-K & -43992.3 & -130240 & -227600 & -277180 \\
\hline Entropy J/kg-K & -2534.29 & -7503.09 & -5672.70 & -6562.69 \\
\hline Density kmol/cum & 0.018 & 0.076 & 101.77 & 42.92 \\
\hline Density kg/cum & 0.314535 & 1.32 & 4083.27 & 1812.58 \\
\hline Average MW & 17.36 & 17.36 & 40.12 & 42.24 \\
\hline Liq Vol 60F cum/hr & 3.04 & & & \\
\hline
\end{tabular}


Table C-12: Mineral carbonation unit energy balance for 66\% extraction and 55\% carbonation

\begin{tabular}{|l|r|r|r|r|r|}
\hline & MGOH & \multicolumn{1}{l|}{ CO2 } & MGOH- & CARB-REC & MGCO-H2 \\
\hline Substream: MIXED & & & & & \\
\hline Mole Flow kmol/hr & & & & & \\
\hline PYROX & 0 & 0 & 0 & 0 & 0 \\
\hline SULFATE & 0 & 0 & 0 & 0 & 0 \\
\hline MGSO4 & 0 & 0 & 0 & 0 & 0 \\
\hline SIO2 & 0 & 0 & 0 & 0 & 0 \\
\hline NH3 & 0 & 0 & 0 & 0 & 0 \\
\hline WATER & 0 & 0 & 0 & 0 & 22.42 \\
\hline MGOH & 24.25 & 0 & 24.25 & 16.51 & 18.34 \\
\hline CO2 & 0 & 22.72 & 0 & 2.75 & 3.05 \\
\hline MGCO3 & 0 & 0 & 0 & 0 & 22.42 \\
\hline Total Flow kmol/hr & 24.25 & 22.72221 & 24.25 & 19.26 & 66.23 \\
\hline Total Flow kg/hr & 1414.25 & 1000.00 & 1414.25 & 1083.89 & 3498.14 \\
\hline Total Flow cum/hr & 0.597 & 555.90 & 0.597 & 8.67 & 77.66 \\
\hline Temperature C & 40 & 25 & 450 & 450 & 450 \\
\hline Pressure atm & 1 & 1 & 1 & 19.74 & 19.74 \\
\hline Vapor Frac & 0 & 1 & 0 & 0.143 & 0.385 \\
\hline Liquid Frac & 0 & 0 & 0 & 0 & 0 \\
\hline Solid Frac & 1 & 0 & 1 & 0.857 & 0.615 \\
\hline Enthalpy kJ/kmol & -923360 & -393510 & -882980 & -810390 & -701080 \\
\hline Enthalpy kJ/kg & -15832.7 & -8941.418 & -15140.29 & -14400.1 & -13274.01 \\
\hline Enthalpy MJ/hr & -22391.43 & -8941.417 & -21412.19 & -15608.05 & -46434.29 \\
\hline Entropy J/kmol-K & -301460 & 2884.454 & -220560 & -186680 & -138190 \\
\hline Entropy J/kg-K & -5169.128 & 65.54118 & -3781.892 & -3317.116 & -2616.453 \\
\hline Density kmol/cum & 40.60089 & 0.0408747 & 40.60089 & 2.22047 & 0.8528946 \\
\hline Density kg/cum & 2367.831 & 1.798887 & 2367.831 & 124.9602 & 45.04669 \\
\hline Average MW & 58.31968 & 44.0098 & 58.31968 & 56.27647 & 52.81625 \\
\hline Liq Vol 60F & & 1.216952 & & & \\
\hline & & & & & \\
\hline
\end{tabular}




\section{C.4. Mineral Acid pH-Swing Process}

Table C-13: Mineral dissolution unit energy balance for 20\% extraction and $65 \%$ carbonation

\begin{tabular}{|c|c|c|c|c|c|c|c|c|}
\hline & PYROX & $\mathrm{HCL}$ & ACID-REC & PYROX-FD & HCL-FD & ACID-RE & PYR-REC & SLURRY-1 \\
\hline \multicolumn{9}{|l|}{ Substream: MIXED } \\
\hline \multicolumn{9}{|l|}{ Mole Flow kmol/hr } \\
\hline PYROXENE & 42 & 0 & 0 & 42 & 0 & 0 & 74.67 & 93.34 \\
\hline $\mathrm{HCL}$ & 0 & 0 & 0 & 0 & 0 & 0 & 0 & 0 \\
\hline MGCL2 & 0 & 0 & 0 & 0 & 0 & 0 & 0 & 0 \\
\hline SIO2 & 0 & 0 & 0 & 0 & 0 & 0 & 93.33 & 116.66 \\
\hline WATER & 0 & 305.56 & 1589.38 & 0 & 305.56 & 1589.38 & 0 & 1918.27 \\
\hline $\mathrm{NAOH}$ & 0 & 0 & 0 & 0 & 0 & 0 & 0 & 0 \\
\hline NACL & 0 & 0 & 0 & 0 & 0 & 0 & 0 & 0 \\
\hline $\mathrm{CO} 2$ & 0 & 0 & 0 & 0 & 0 & 0 & 0 & 0 \\
\hline MGCO3 & 0 & 0 & 0 & 0 & 0 & 0 & 0 & 0 \\
\hline MG++ & 0 & 0 & 0 & 0 & 0 & 0 & 0 & 23.33 \\
\hline $\mathrm{H+}$ & 0 & 11 & 35.74 & 0 & 11 & 35.74 & 0 & 0.072 \\
\hline NA+ & 0 & 0 & 0 & 0 & 0 & 0 & 0 & 0 \\
\hline HCO3- & 0 & 0 & 0 & 0 & 0 & 0 & 0 & 0 \\
\hline $\mathrm{OH}-$ & 0 & $7.22 \mathrm{E}-14$ & $5.22 \mathrm{E}-13$ & 0 & $9.65 \mathrm{E}-13$ & $9.93 \mathrm{E}-12$ & 0 & 3.39E-09 \\
\hline CL- & 0 & 11 & 35.74 & 0 & 11 & 35.74 & 0 & 46.74 \\
\hline CO3-- & 0 & 0 & 0 & 0 & 0 & 0 & 0 & 0 \\
\hline Total Flow kmol/hr & 42 & 327.56 & 1660.86 & 42 & 327.56 & 1660.86 & 168 & 2198.42 \\
\hline Total Flow kg/hr & 4216.33 & 5905.74 & 29936.23 & 4216.33 & 5905.74 & 29936.23 & 13103.69 & 53161.98 \\
\hline Total Flow cum/hr & 1.26 & 5.92 & 29.98 & 1.26 & 6.04 & 30.61 & 4.36 & 41.19 \\
\hline Temperature C & 25 & 25 & 20 & 70 & 70 & 70 & 70 & 70 \\
\hline Pressure atm & 1 & 1 & 1 & 1 & 1 & 1 & 1 & 1 \\
\hline Vapor Frac & 0 & 0 & 0 & 0 & 0 & 0 & 0 & 0 \\
\hline Liquid Frac & 0 & 1 & 1 & 0 & 1 & 1 & 0 & 0.904 \\
\hline Solid Frac & 1 & 0 & 0 & 1 & 0 & 0 & 1 & 0.096 \\
\hline Enthalpy kJ/kmol & -1548900 & -272500 & -277690 & -1545100 & -269440 & -274180 & -1191500 & -369050 \\
\hline Enthalpy kJ/kg & -15429.2 & -15113.86 & -15406.39 & -15390.95 & -14944.1 & -15211.3 & -15275.8 & -15261.5 \\
\hline Enthalpy kW & -18070.7 & -24794.02 & -128110 & -18025.9 & -24515.5 & -126490 & -55602.6 & -225370 \\
\hline Entropy J/kmol-K & -291450 & -156090 & -159630 & -279470 & -146530 & -148550 & -221940 & -157130 \\
\hline Entropy J/kg-K & -2903.2 & -8657.548 & -8856.015 & \begin{tabular}{|l|}
-2783.832 \\
\end{tabular} & -8127.22 & -8241.52 & -2845.42 & -6497.67 \\
\hline Density kmol/cum & 33.33 & 55.30 & 55.41 & 33.33 & 54.22 & 54.26 & 38.55 & 53.37 \\
\hline Density kg/cum & 3346.29 & 997.01 & 998.67 & 3346.29 & 977.54 & 978.03 & 3007.11 & 1290.71 \\
\hline Average MW & 100.39 & 18.03 & 18.02 & 100.39 & 18.03 & 18.02 & 78.00 & 24.18 \\
\hline Liq Vol 60F & & & & & & & & \\
\hline
\end{tabular}


Table C-14: pH adjustment unit energy balance for 20\% extraction and 65\% carbonation

\begin{tabular}{|l|r|r|r|}
\hline & LEACH-1 & NAOH & LEACH-2 \\
\hline Substream: MIXED & & & \\
\hline Mole Flow kmol/hr & & & \\
\hline PYROXENE & 0 & 0 & 0 \\
\hline HCL & 0 & 0 & 0 \\
\hline MGCL2 & 0 & 0 & 0 \\
\hline SIO2 & 0 & 0 & 0 \\
\hline WATER & 1918.27 & 3.704 & 1922.05 \\
\hline NAOH & 0 & 0 & 0 \\
\hline NACL & 0 & 0 & 0 \\
\hline CO2 & 0 & 0 & 0 \\
\hline MGCO3 & 0 & 0 & 0 \\
\hline MG++ & 23.33 & 0 & 23.33 \\
\hline H+ & 0.074 & $2.91 \mathrm{E}-18$ & $1.27 \mathrm{E}-11$ \\
\hline NA+ & 0 & 1 & 1 \\
\hline HCO3- & 0 & 0 & 0 \\
\hline OH- & $3.30 \mathrm{E}-09$ & 1 & 0.926 \\
\hline CL- & 46.73 & 0 & 46.73 \\
\hline CO3-- & 0 & 0 & 0 \\
\hline Total Flow kmol/hr & 1988.41 & 5.704 & 1994.04 \\
\hline Total Flow kg/hr & 36782.13 & 106.7257 & 36888.85 \\
\hline Total Flow cum/hr & 35.74 & 0.0761 & 35.07 \\
\hline Temperature C & 70 & 25 & 20 \\
\hline Pressure atm & 1 & 1 & 1 \\
\hline Vapor Frac & 0 & 0 & 0 \\
\hline Liquid Frac & 1 & 1 & 1 \\
\hline Solid Frac & 0 & 0 & 0 \\
\hline Enthalpy kJ/kmol & -282190 & -266600 & -285650 \\
\hline Enthalpy kJ/kg & -15255.17 & -14248.65 & -15441.11 \\
\hline Enthalpy kW & -155870 & -422.416 & -158220 \\
\hline Entropy J/kmol-K & -150280 & -136210 & -161180 \\
\hline Entropy J/kg-K & -8124.094 & -7279.591 & -8712.732 \\
\hline Density kmol/cum & 55.63 & 74.92 & 56.86 \\
\hline Density kg/cum & 1029.12 & 1401.79 & 1051.98 \\
\hline Average MW & 18.50 & 18.71 & 18.50 \\
\hline Liq Vol 60F & & & \\
\hline
\end{tabular}


Table C-15: Mineral carbonation unit energy balance for 20\% extraction and 65\% carbonation

\begin{tabular}{|l|r|r|r|r|}
\hline & LEACH-2 & \multicolumn{1}{l|}{ CO2 } & \multicolumn{1}{l|}{ SOL-REC } & SLURRY-2 \\
\hline Substream: MIXED & & & & \\
\hline Mole Flow kmol/hr & & & & \\
\hline PYROXENE & 0 & 0 & 0 & 0 \\
\hline HCL & 0 & 0 & 0 & 0 \\
\hline MGCL2 & 0 & 0 & 0 & 0 \\
\hline SIO2 & 0 & 0 & 0 & 0 \\
\hline WATER & 1922.05 & 0 & 176.78 & 2087.10 \\
\hline NAOH & 0 & 0 & 0 & 0 \\
\hline NACL & 0 & 0 & 0 & 0 \\
\hline CO2 & 0 & 22.72 & 4.55 & 14.62 \\
\hline H2CO3 & 0 & 0 & 0 & 0 \\
\hline MGCO3 & 0 & 0 & 0 & 17.73 \\
\hline MG++ & 23.33 & 0 & 13.09 & 18.69 \\
\hline H+ & $3.99 E-11$ & 0 & $9.41 \mathrm{E}-09$ & 29.27 \\
\hline NA+ & 0.9997 & 0 & 5.67 & 6.67 \\
\hline HCO3- & 0 & 0 & 4.90 & $4.43 \mathrm{E}-07$ \\
\hline OH- & 0.9257 & 0 & $1.24 \mathrm{E}-06$ & $1.40 \mathrm{E}-12$ \\
\hline CL- & 46.734 & 0 & 26.60 & 73.34 \\
\hline CO3-- & 0 & 0 & 0.177 & $4.46 \mathrm{E}-16$ \\
\hline Total Flow kmol/hr & 1994.04 & 22.72 & 231.76 & 2247.42 \\
\hline Total Flow kg/hr & 36888.88 & 1000 & 5085.77 & 42974.64 \\
\hline Total Flow cum/hr & 35.11 & 6.68 & 97.95 & 40.50 \\
\hline Temperature C & 25 & 35 & 20 & 20 \\
\hline Pressure atm & 1 & 57.48 & 1 & 1 \\
\hline Vapor Frac & 0 & 1 & 0.017 & 0 \\
\hline Liquid Frac & 1 & 0 & 0.983 & 0.992 \\
\hline Solid Frac & 0 & 0 & 0 & 0.008 \\
\hline Enthalpy kJ/kmol & -285270 & -396040 & -291130 & -287400 \\
\hline Enthalpy kJ/kg & -15420.45 & -8998.79 & -13266.96 & -15029.73 \\
\hline Enthalpy kW & -158010 & -2499.67 & -18742.4 & -179420 \\
\hline Entropy J/kmol-K & -160150 & -35676.65 & -144930 & -158520 \\
\hline Entropy J/kg-K & -8657.06 & -810.65 & -6604.54 & -8290.18 \\
\hline Density kmol/cum & 56.80 & 3.40 & 2.37 & 55.50 \\
\hline Density kg/cum & 1050.75 & 149.71 & 51.92 & 1061.20 \\
\hline Average MW & 18.50 & 44.01 & 21.94 & 19.12 \\
\hline Liq Vol 60F & 1.22 & & \\
\hline
\end{tabular}




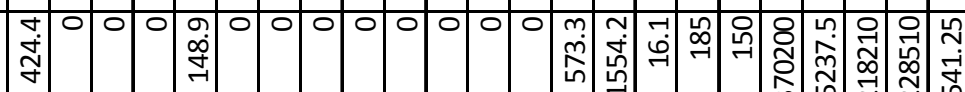

ᄁ)

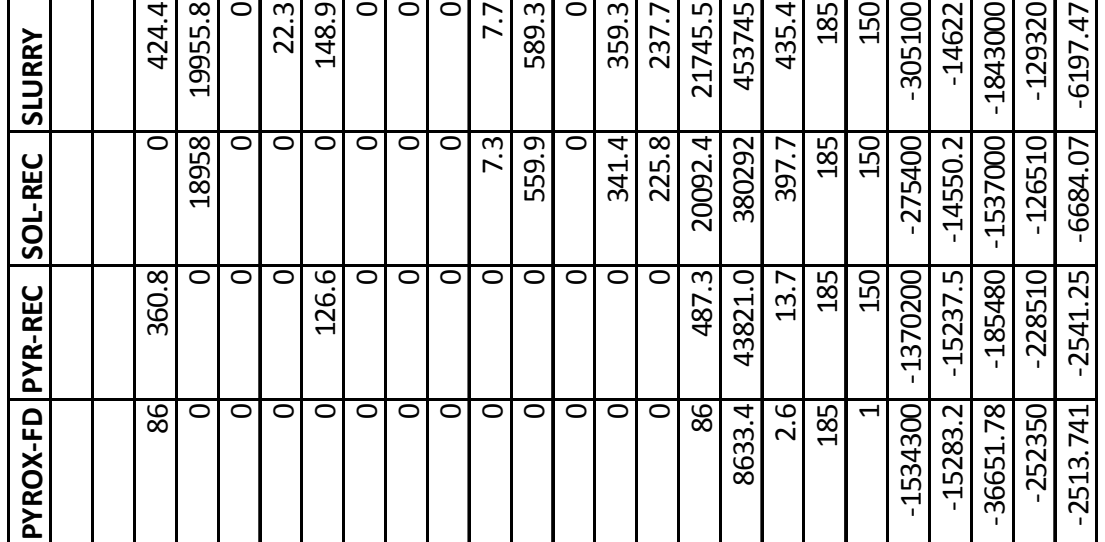

ชิ่

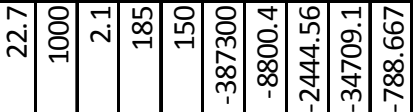
10

ํำ

今.

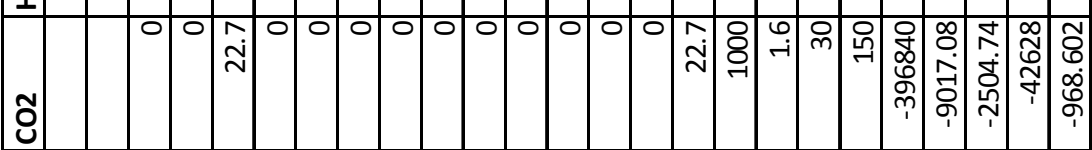

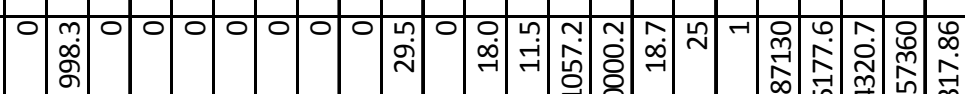
ำ

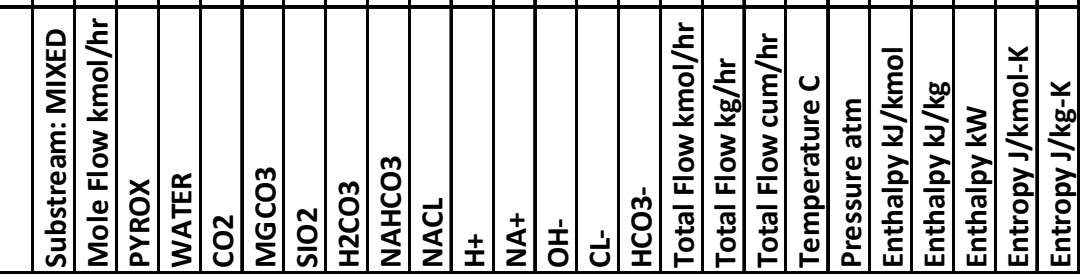




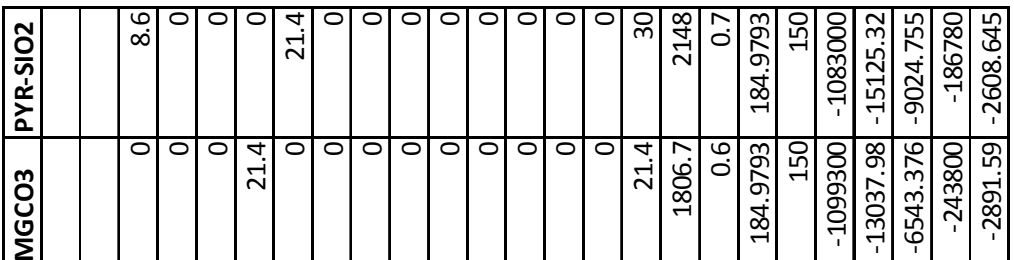

요

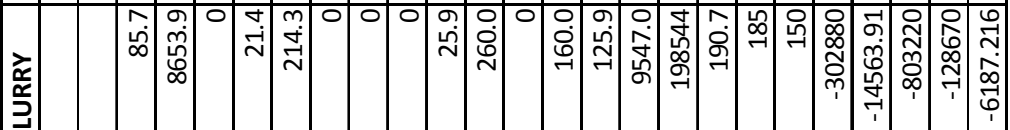

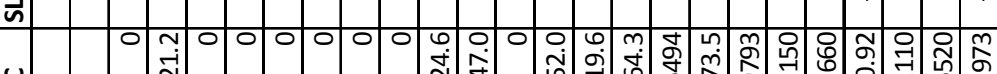

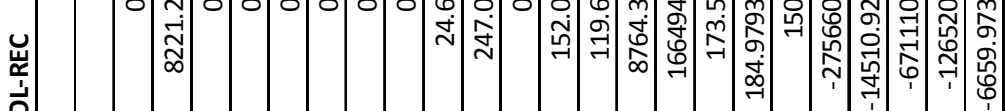

它

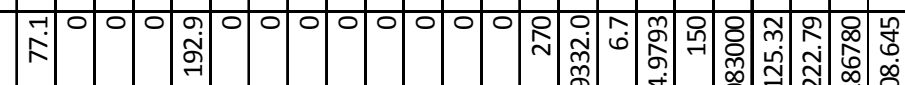

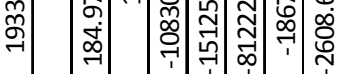

究
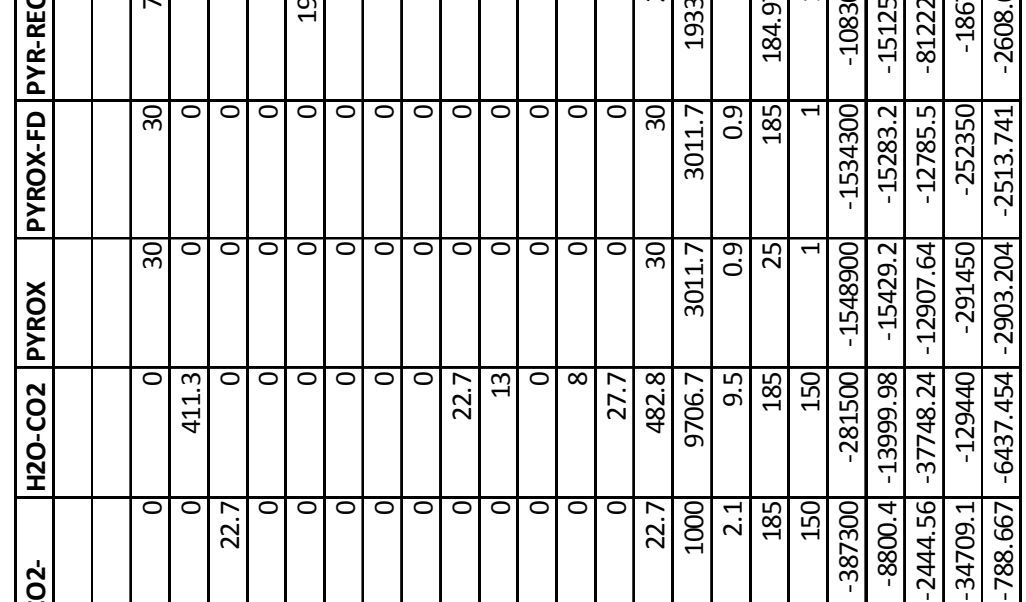

ธิ่

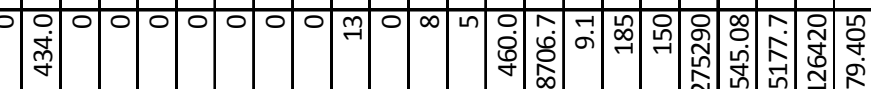

ํำ

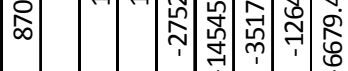

ชิ

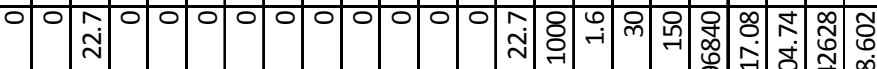

ำ
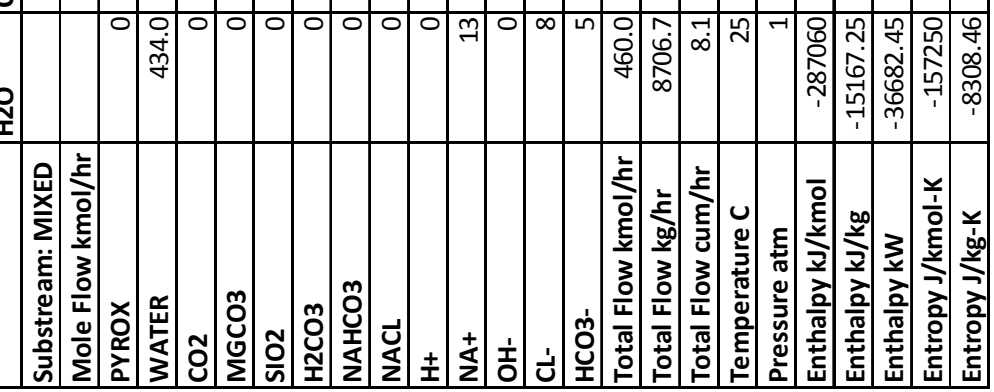


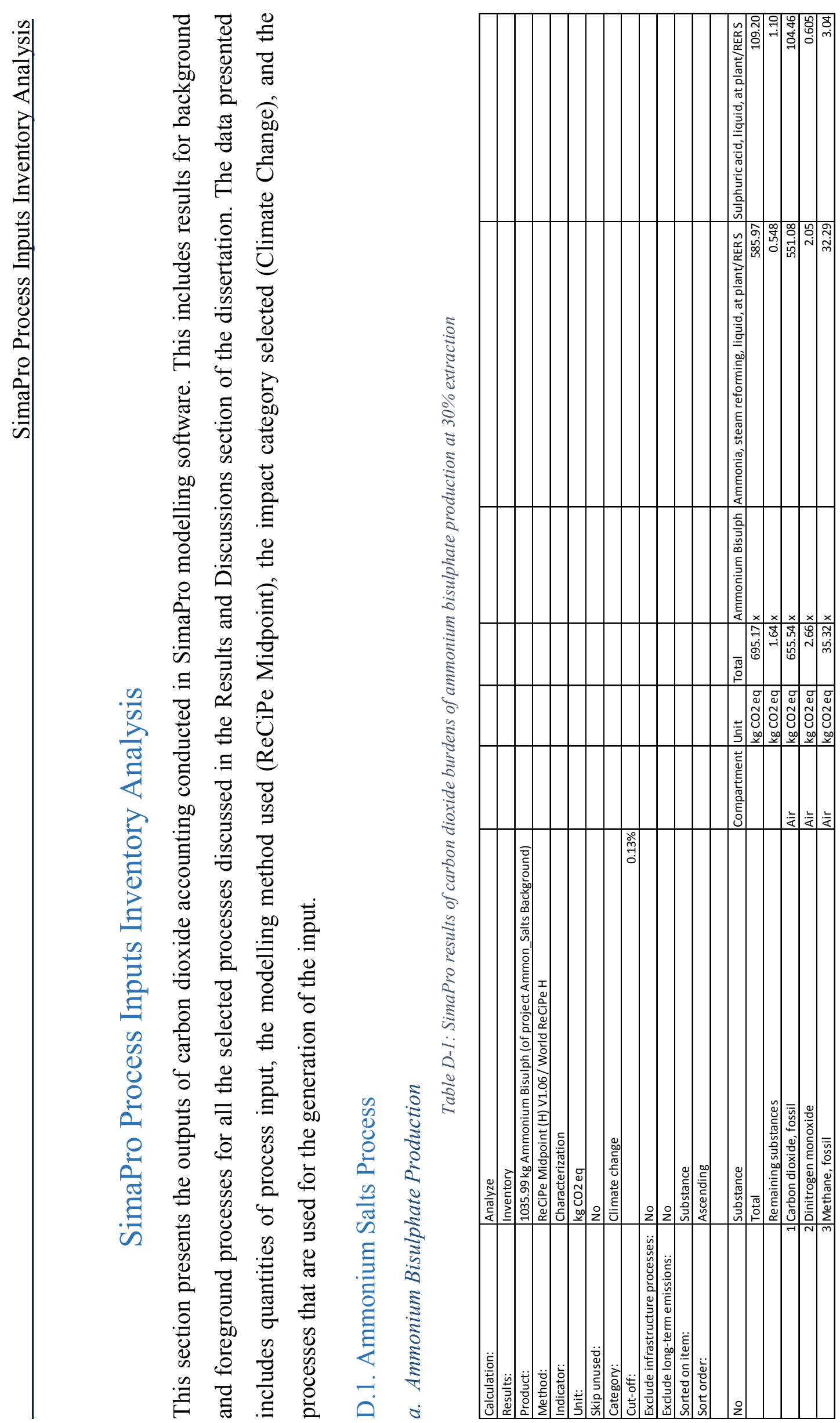



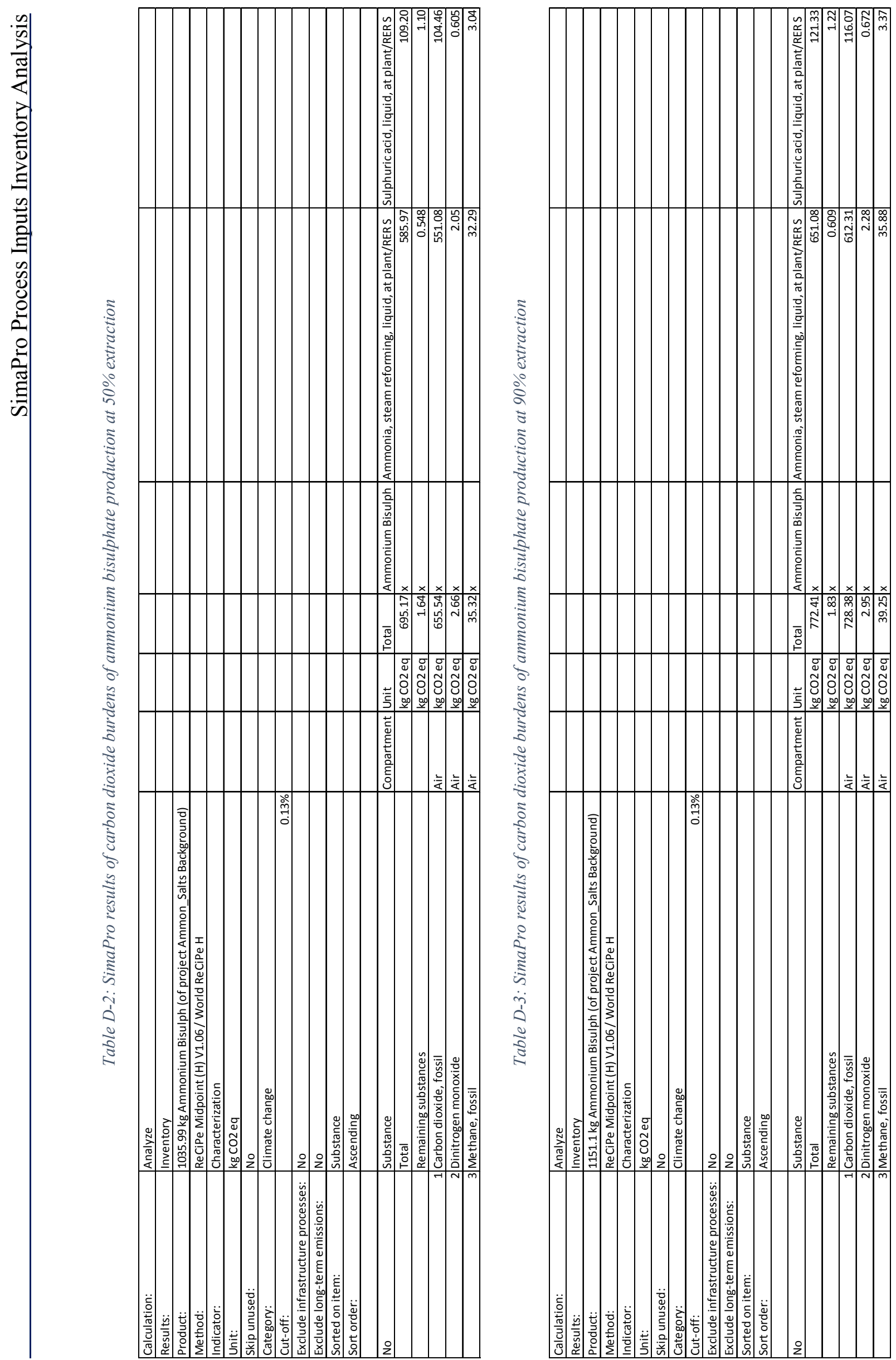
告
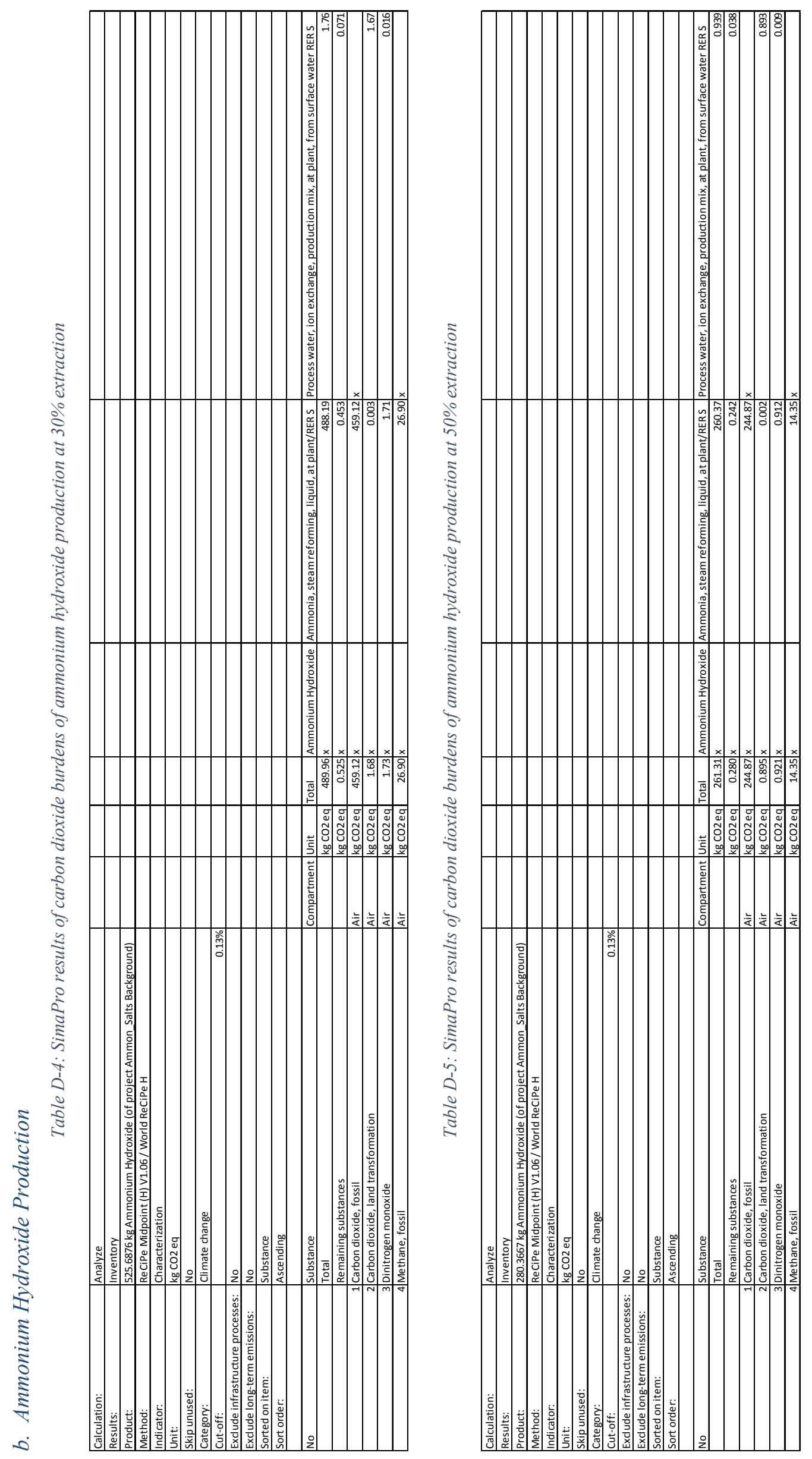
|ân
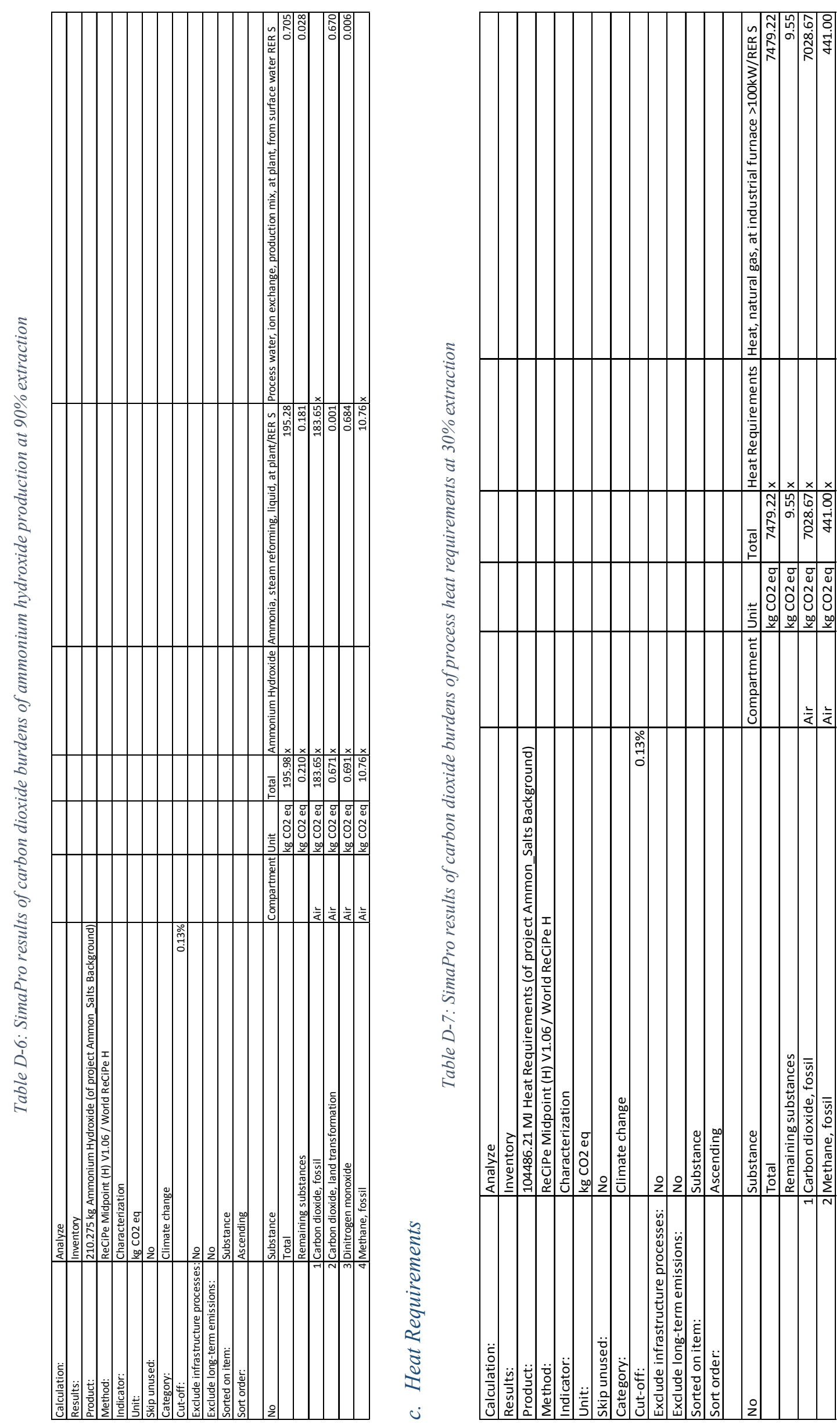


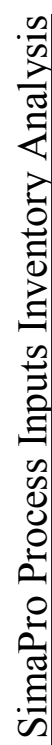
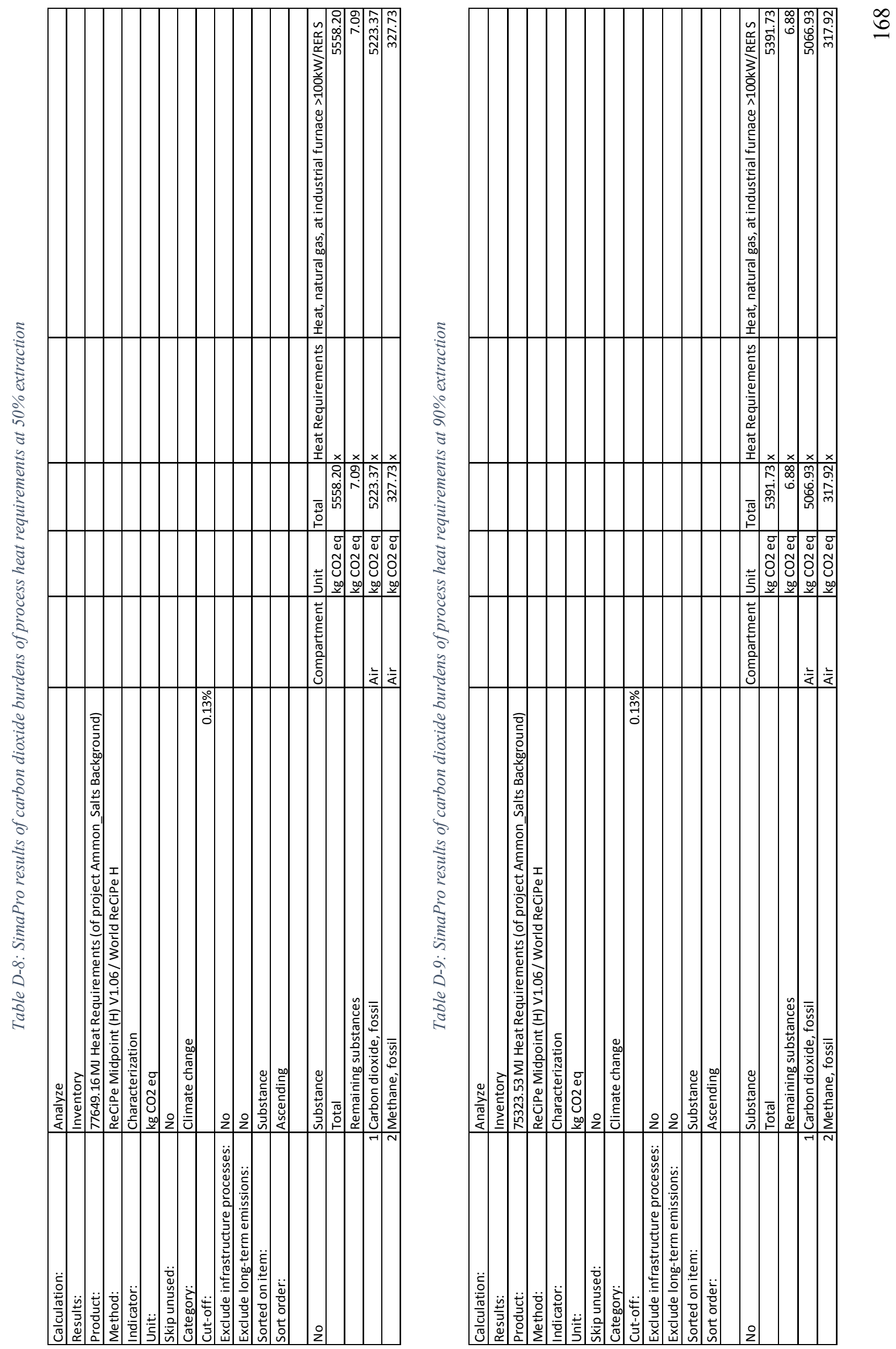
|ân
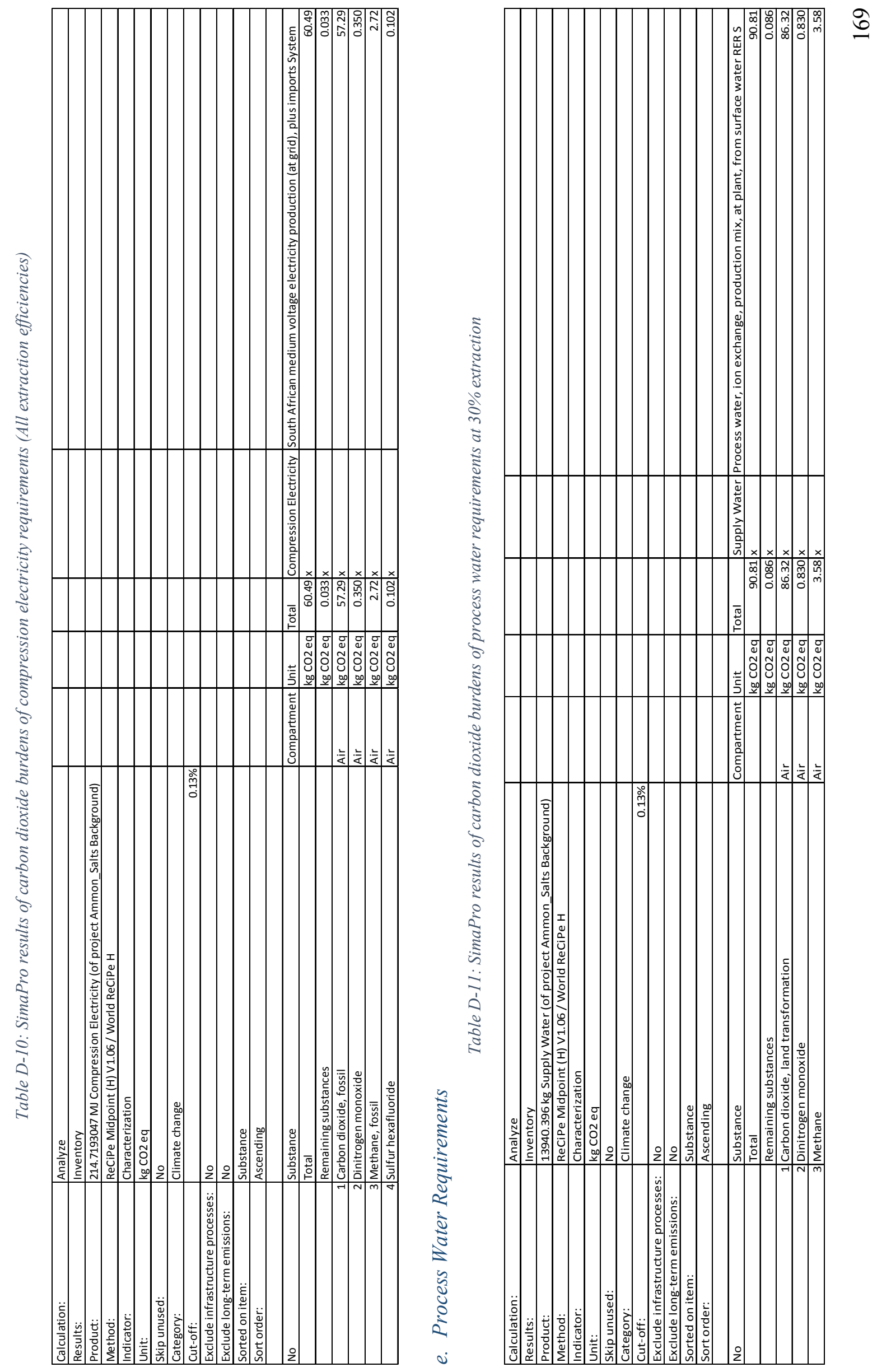

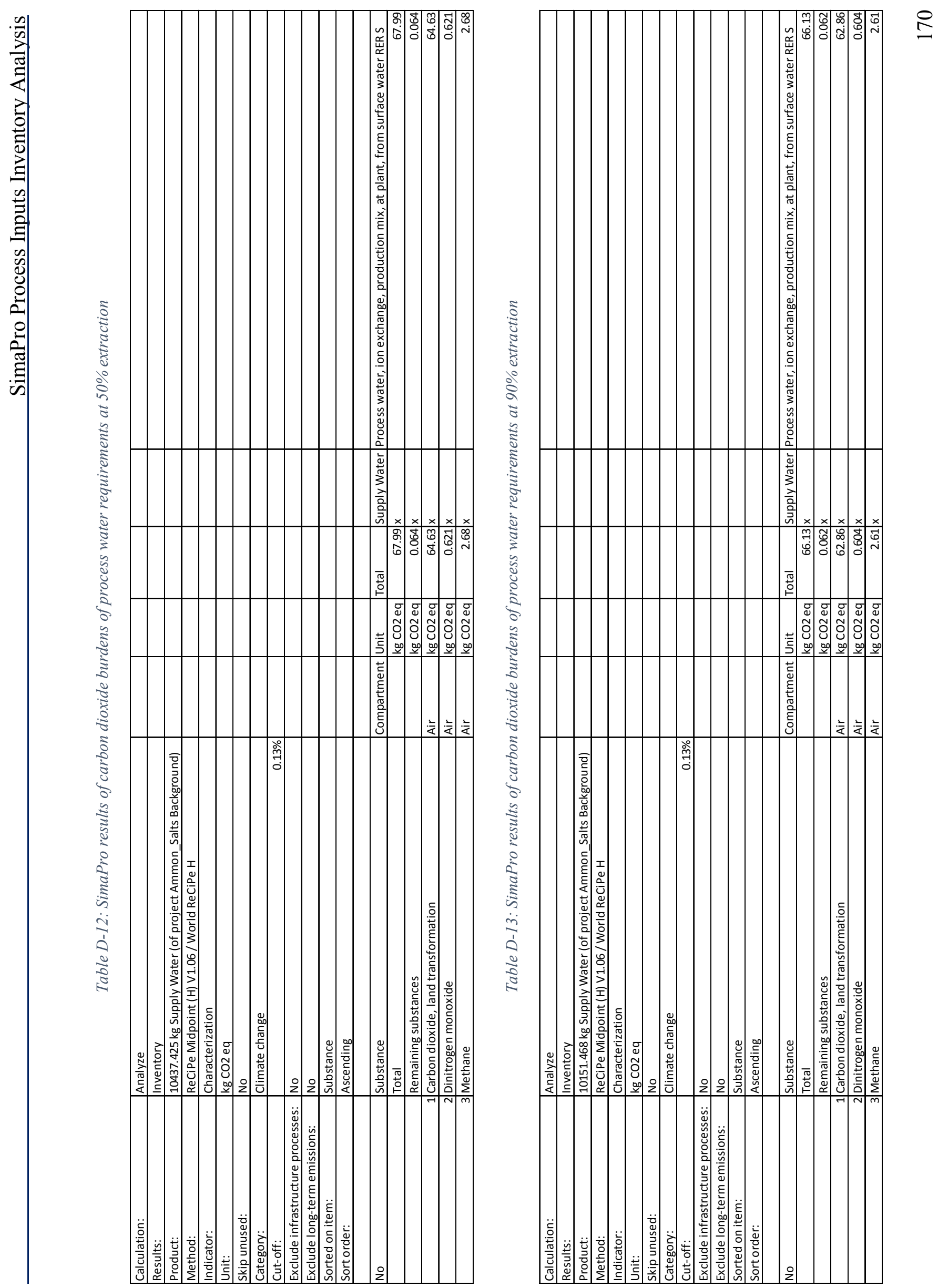


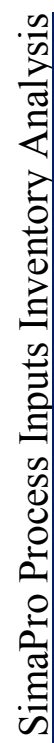

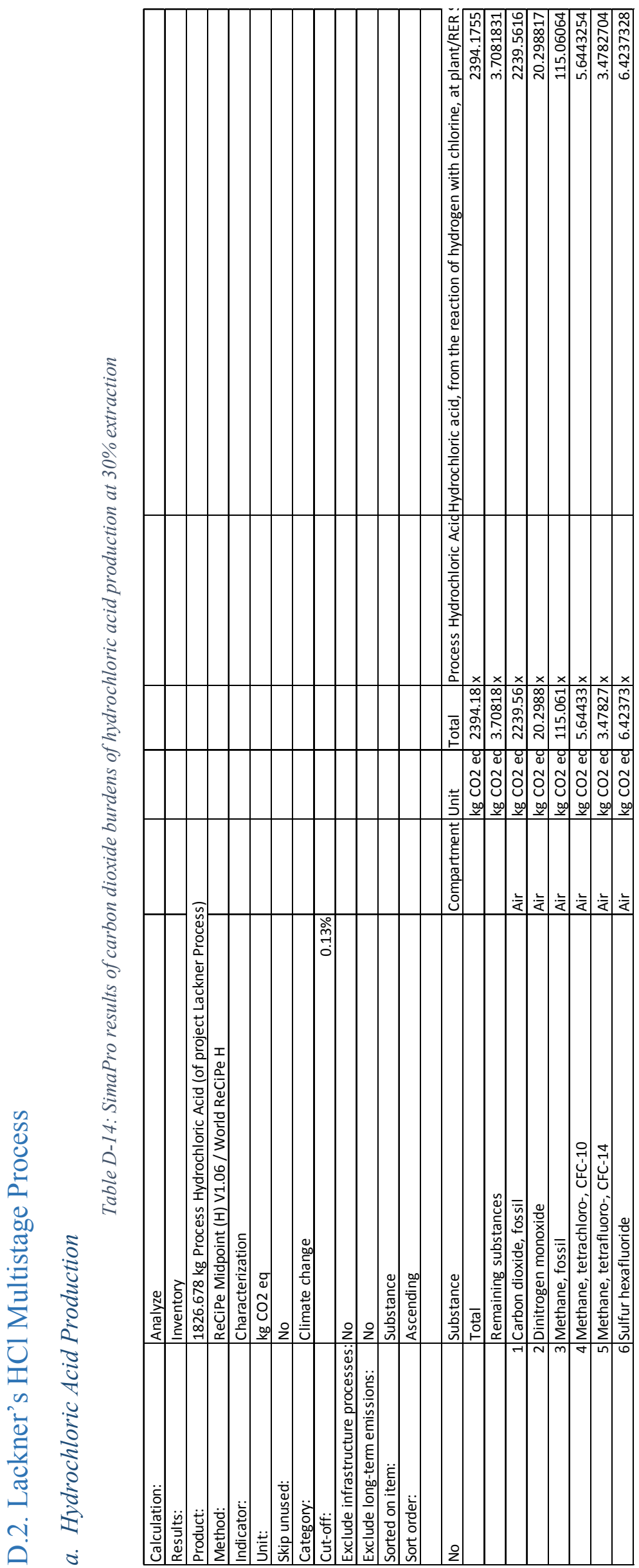




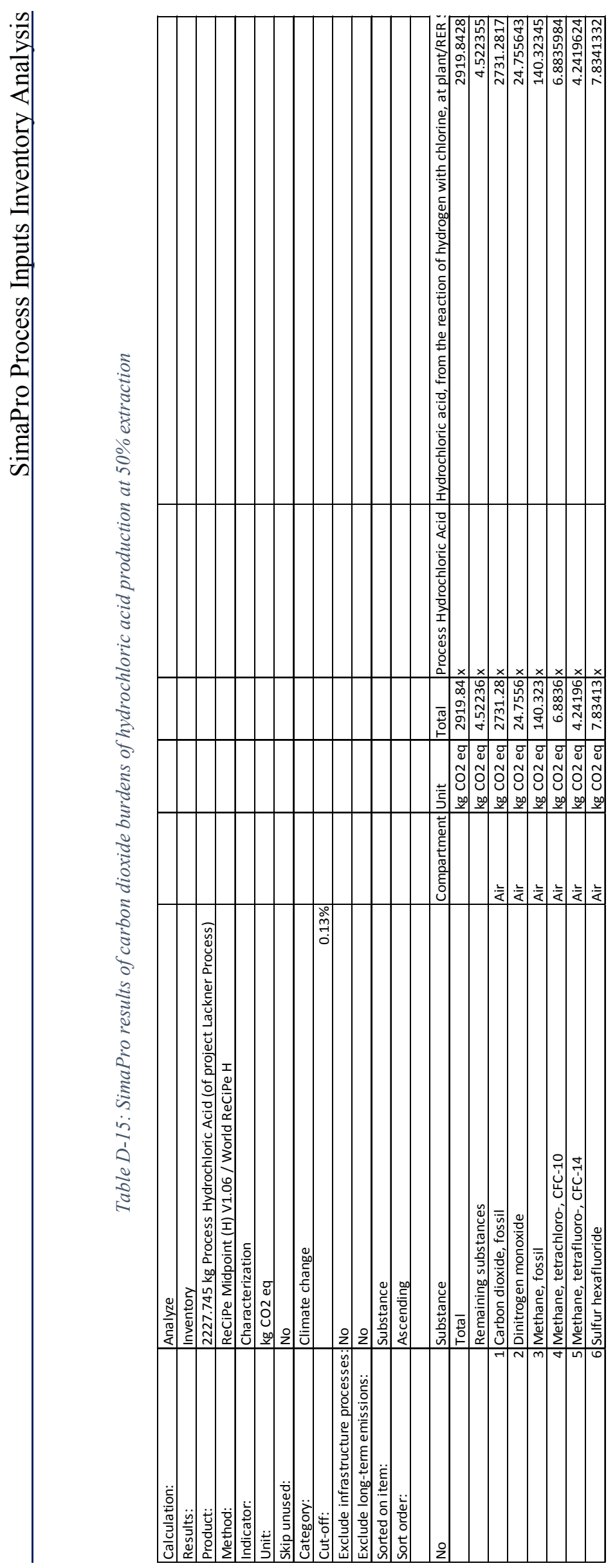




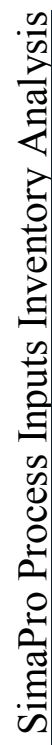
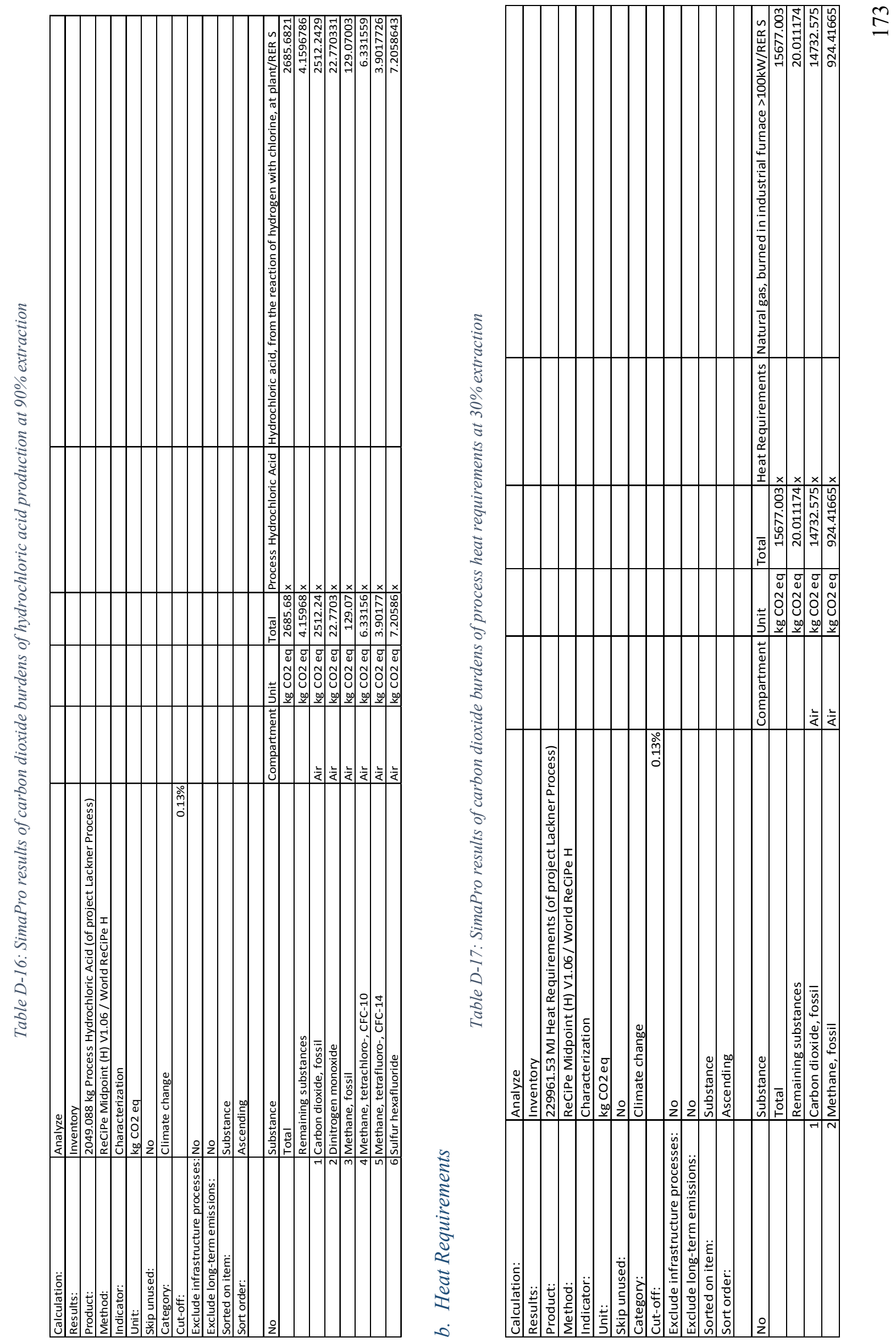
.
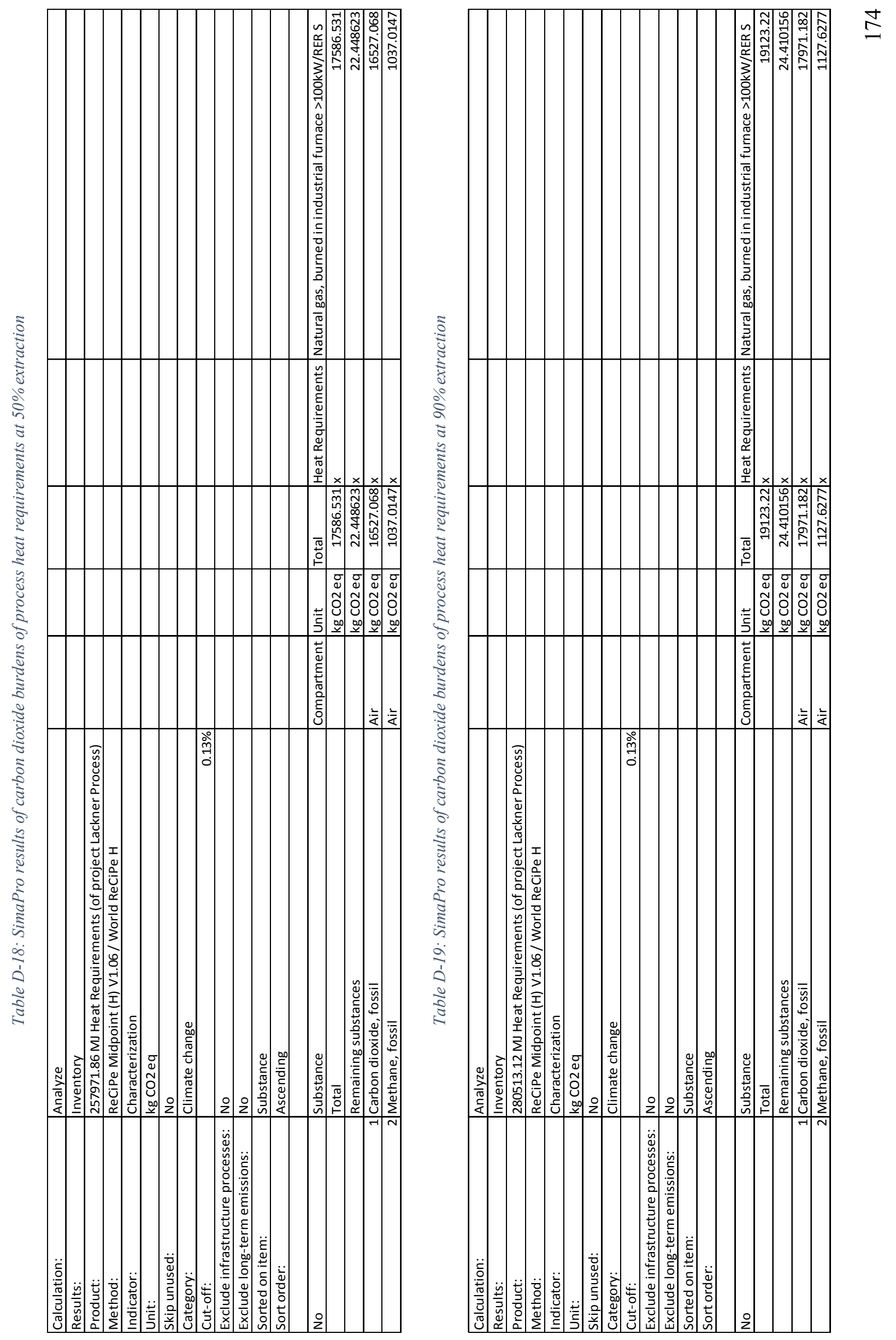
.
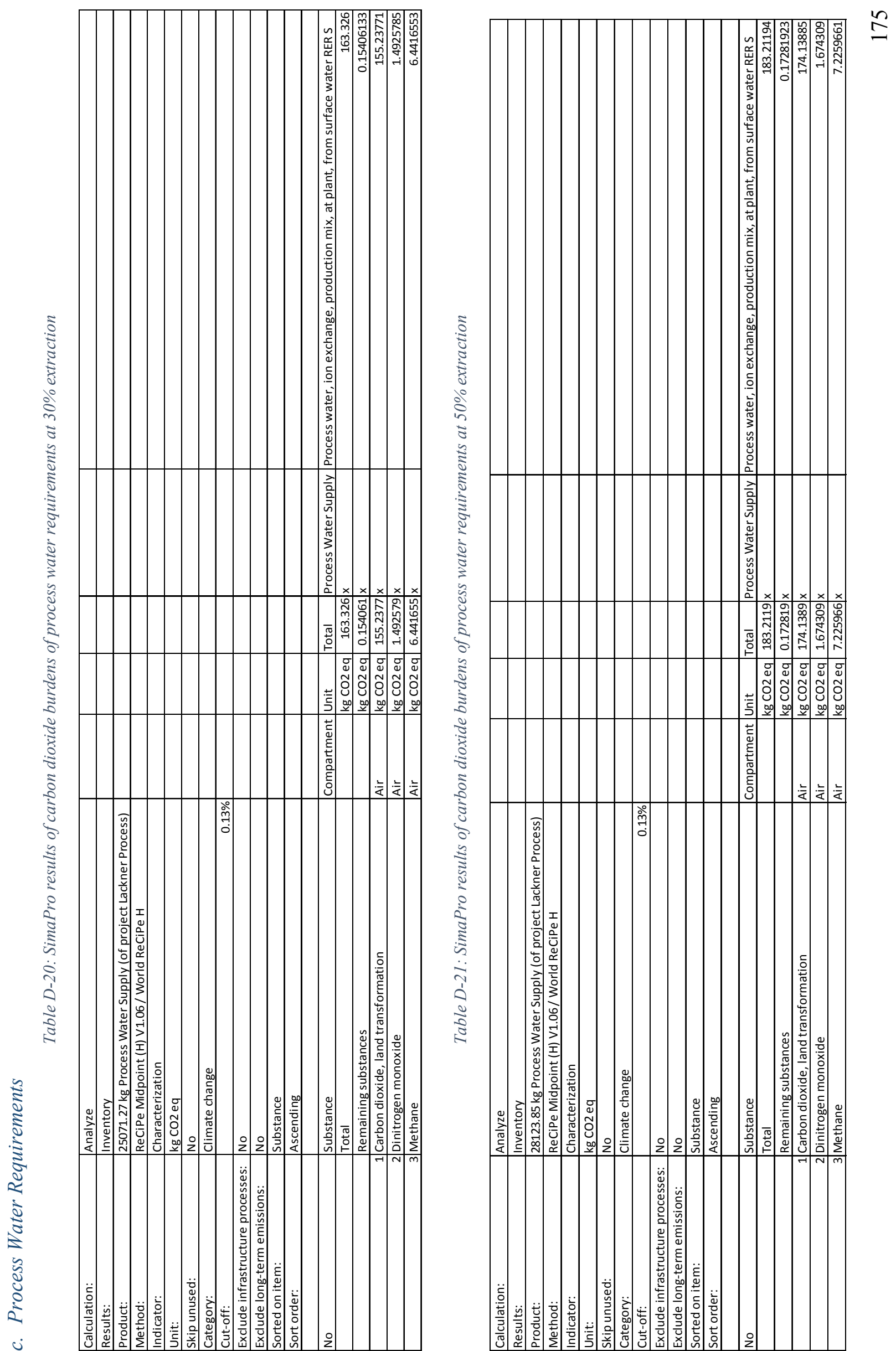

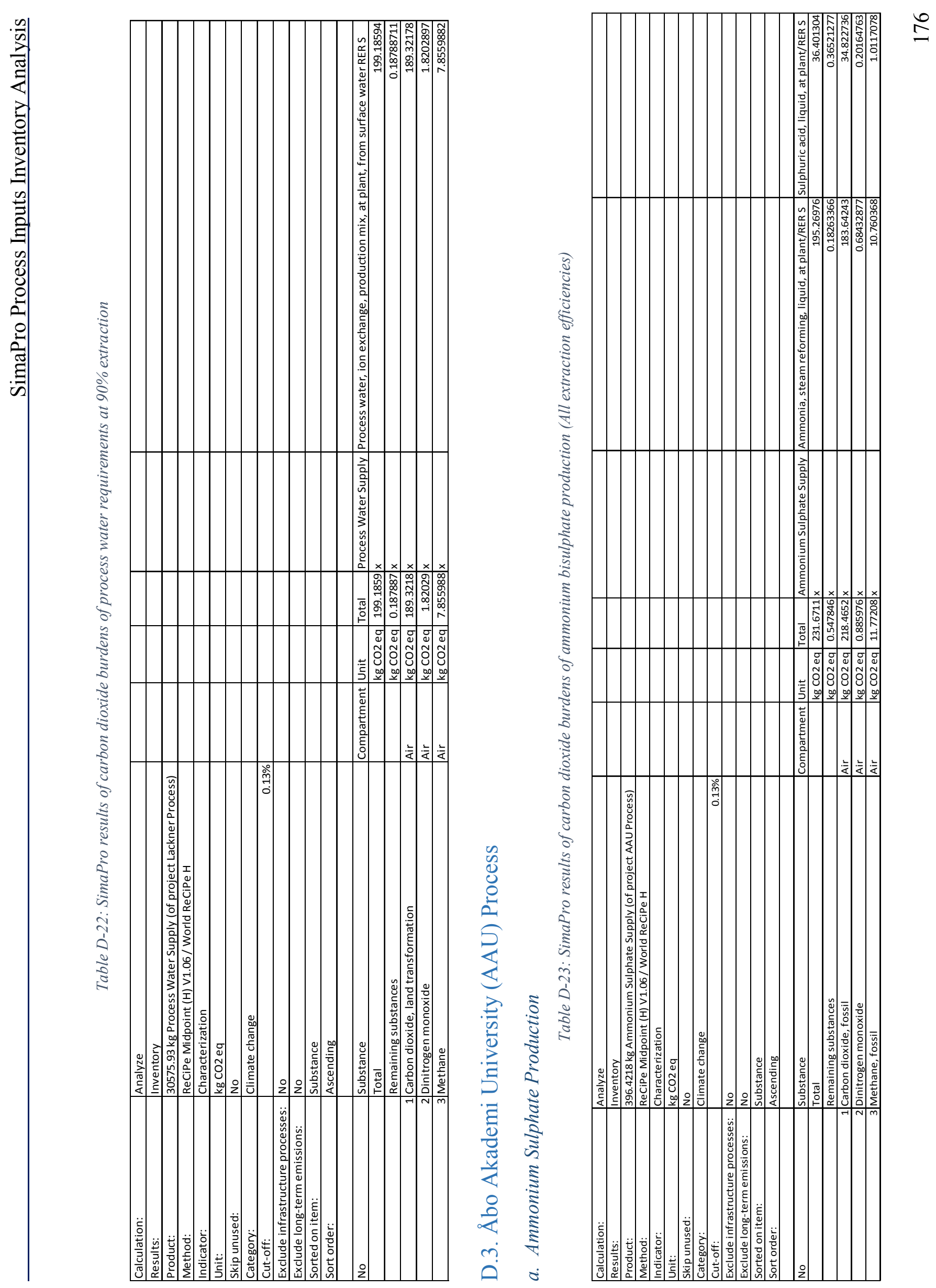
.
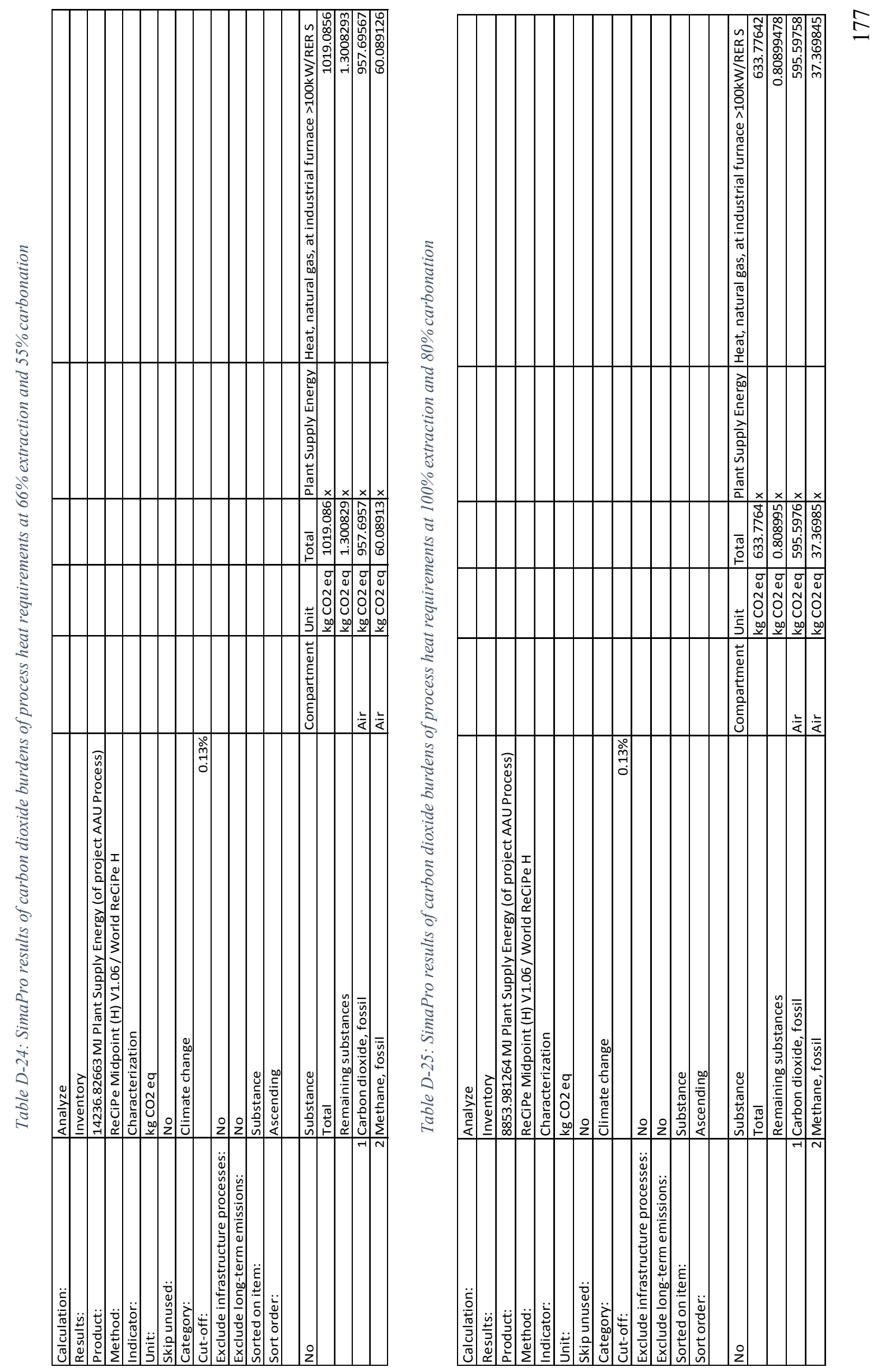
|ân
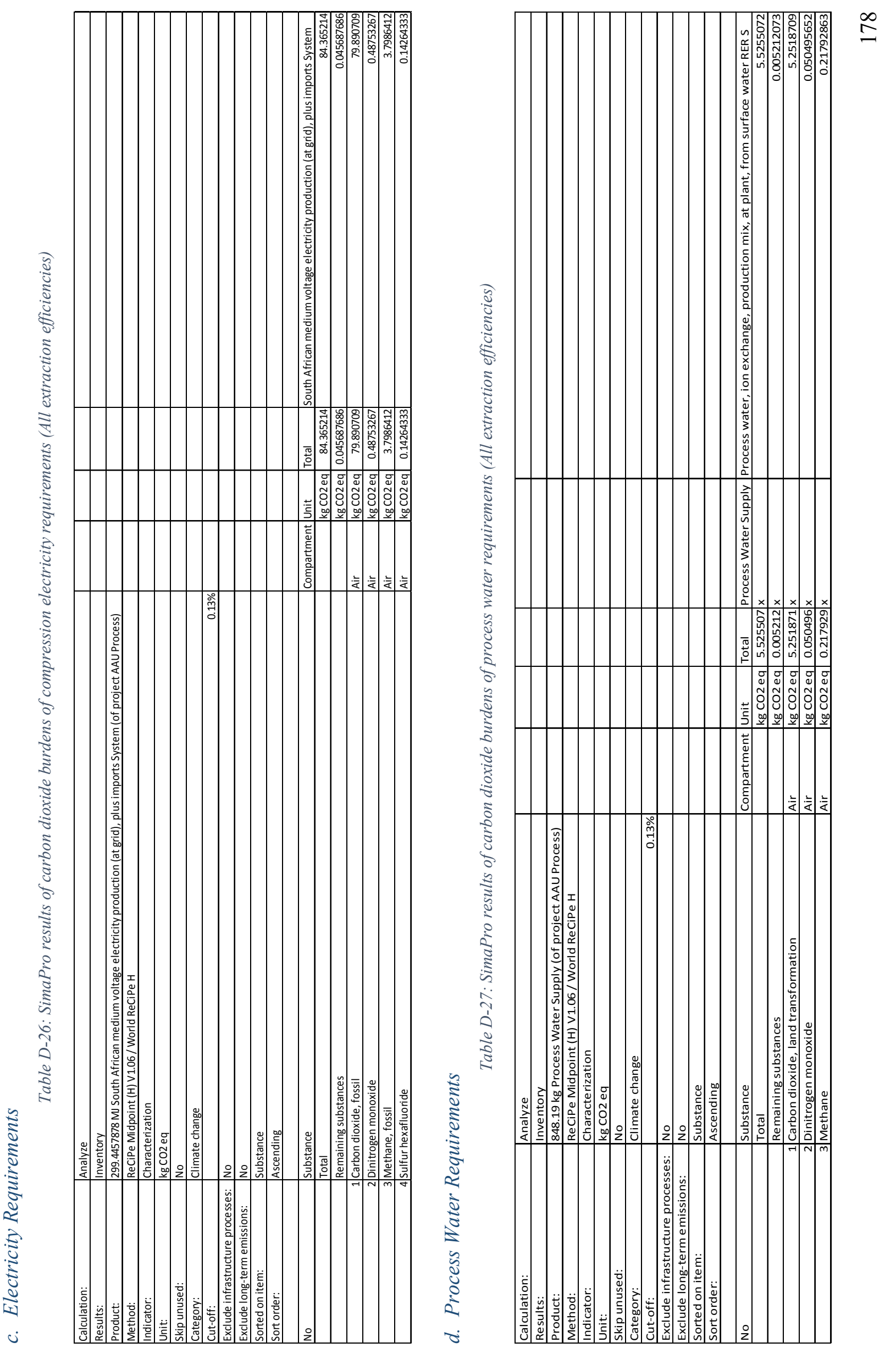


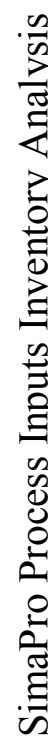

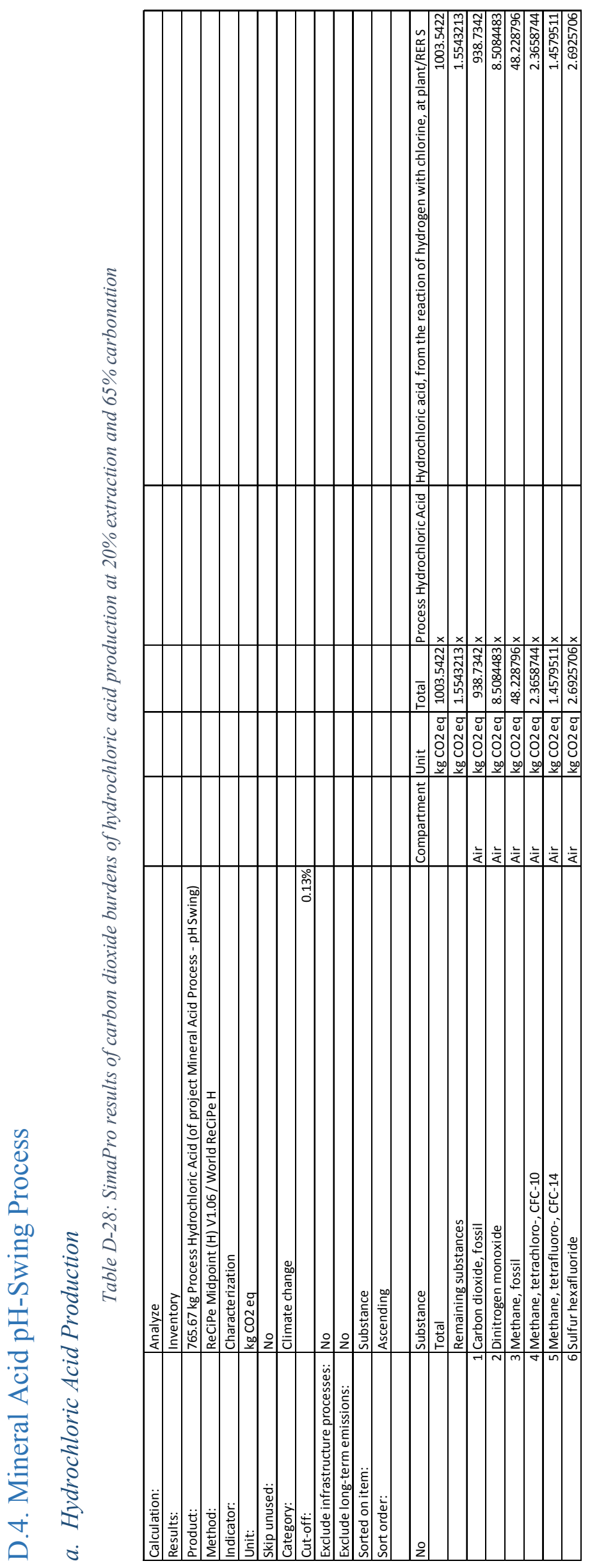

$\stackrel{2}{2}$ 


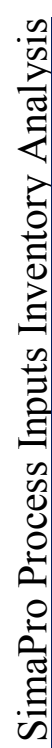
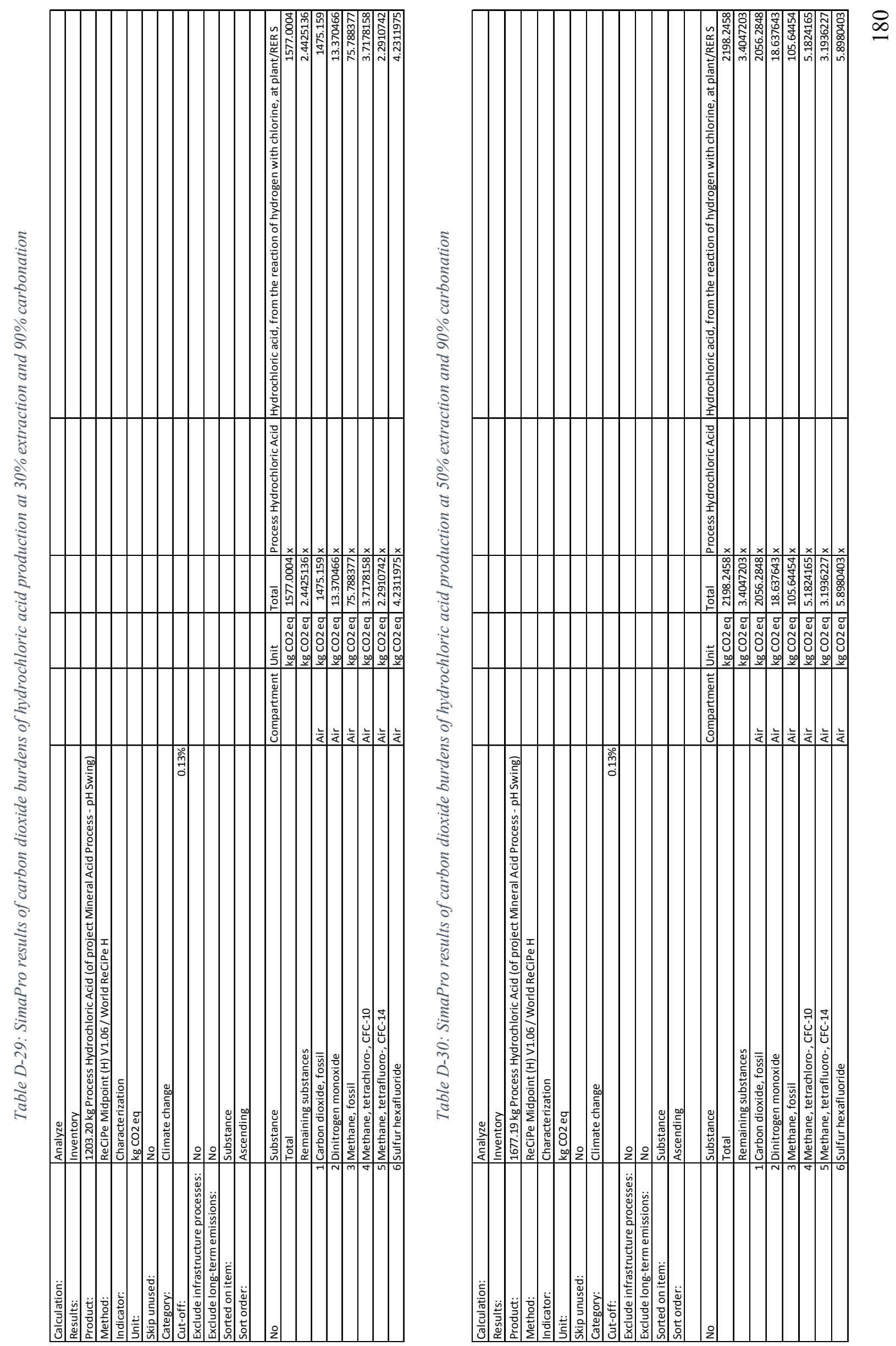
告

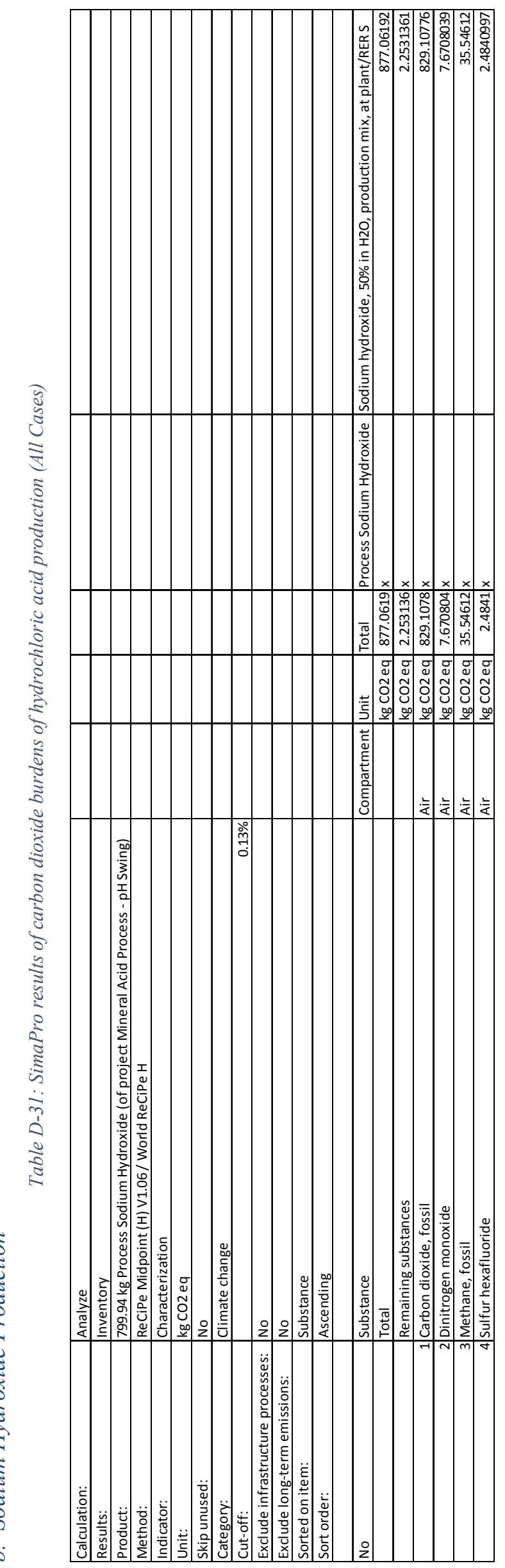


|a
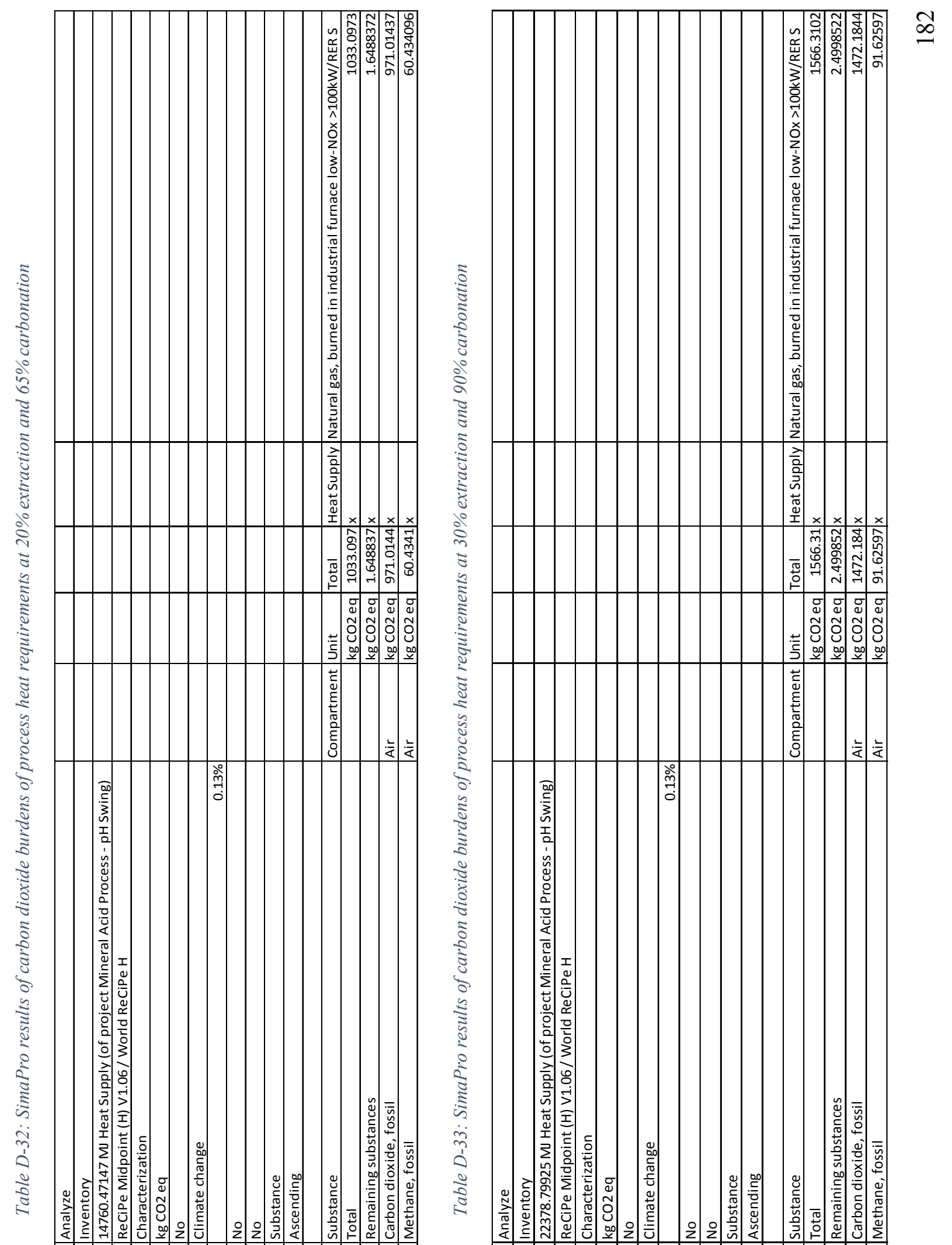
|⿹

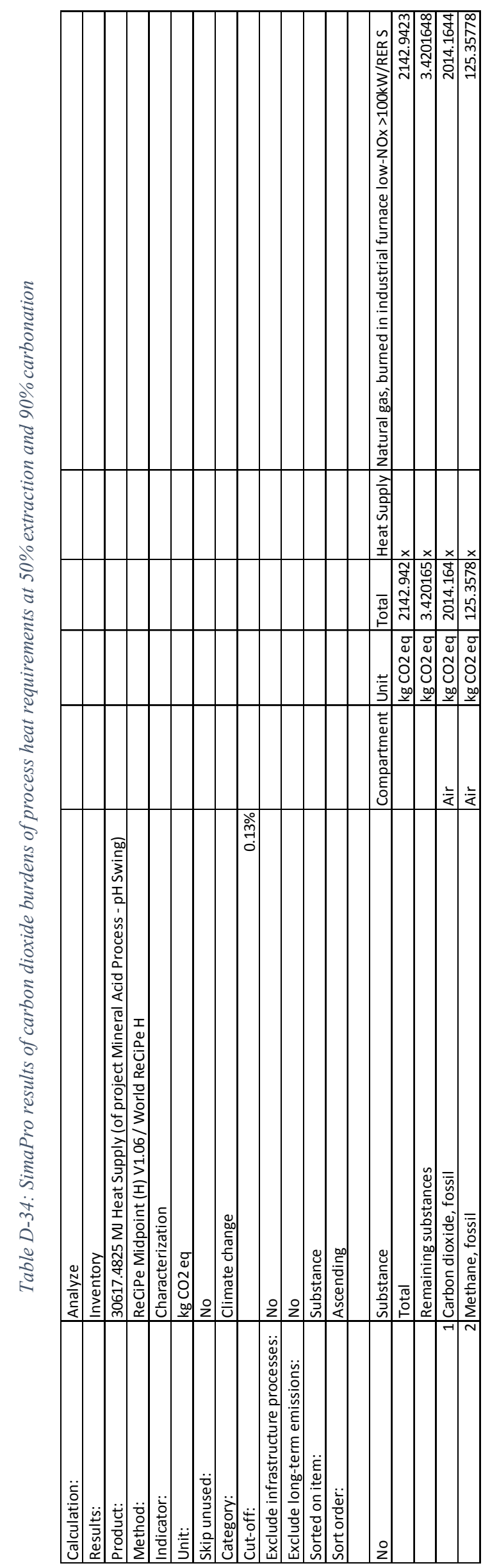

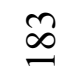


.
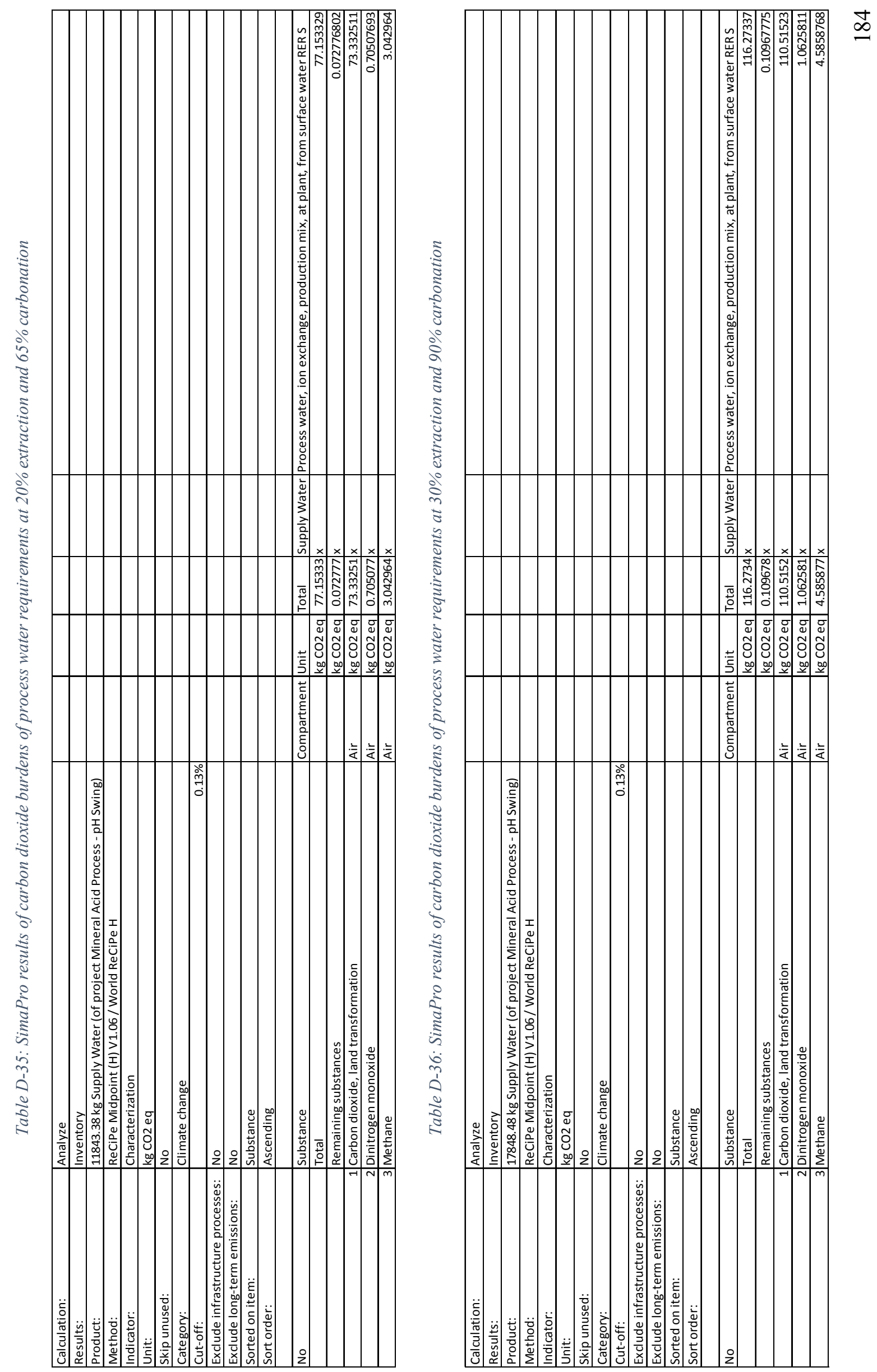


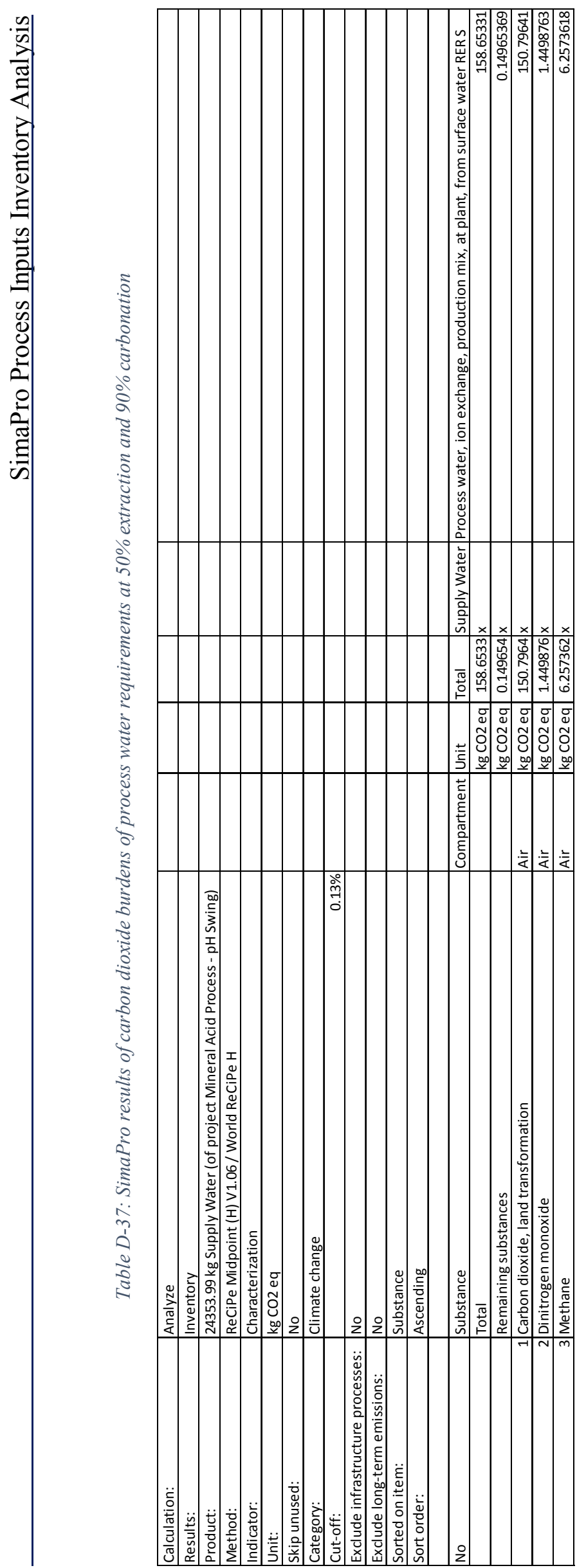




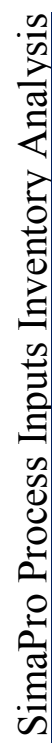
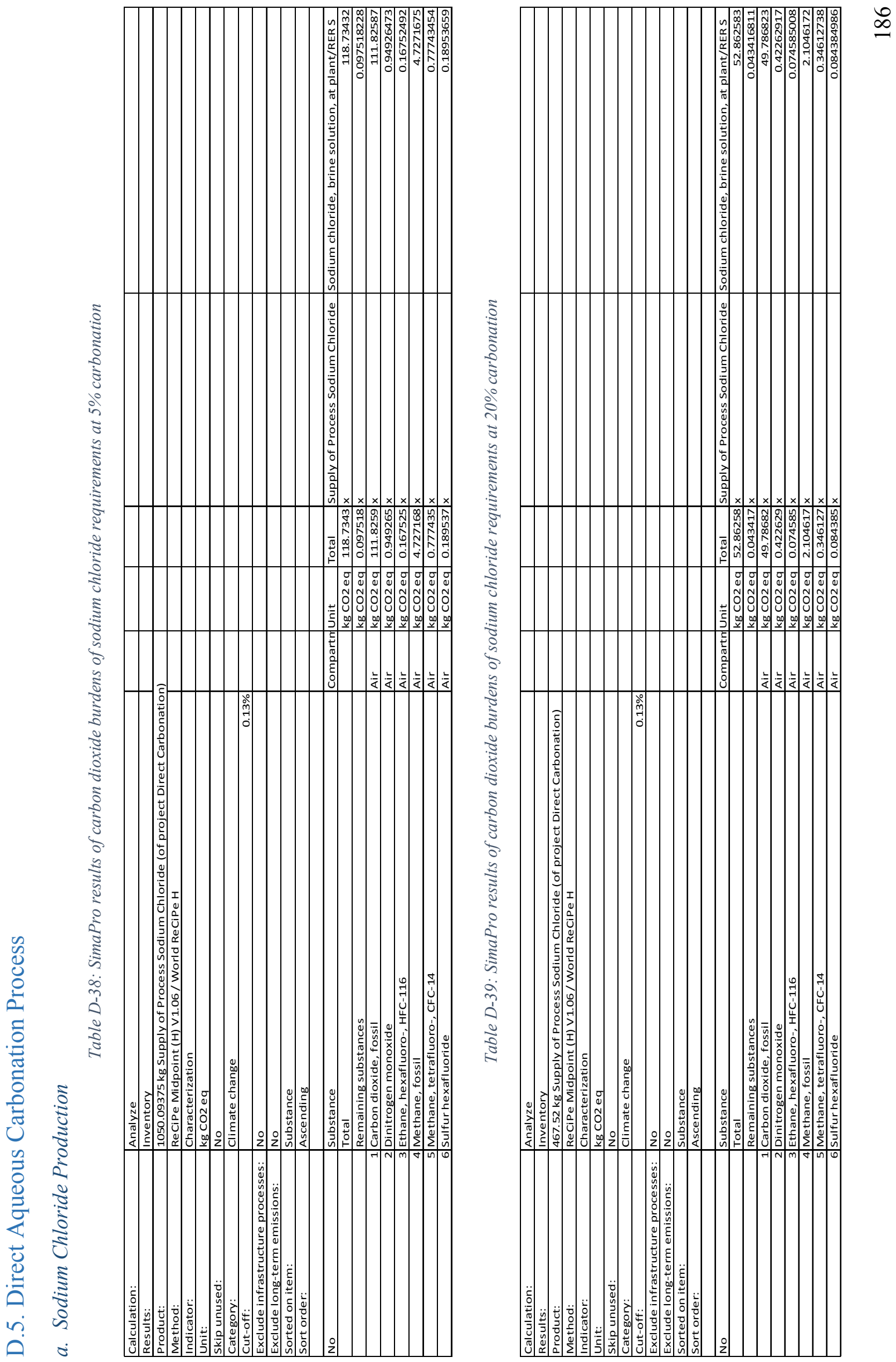

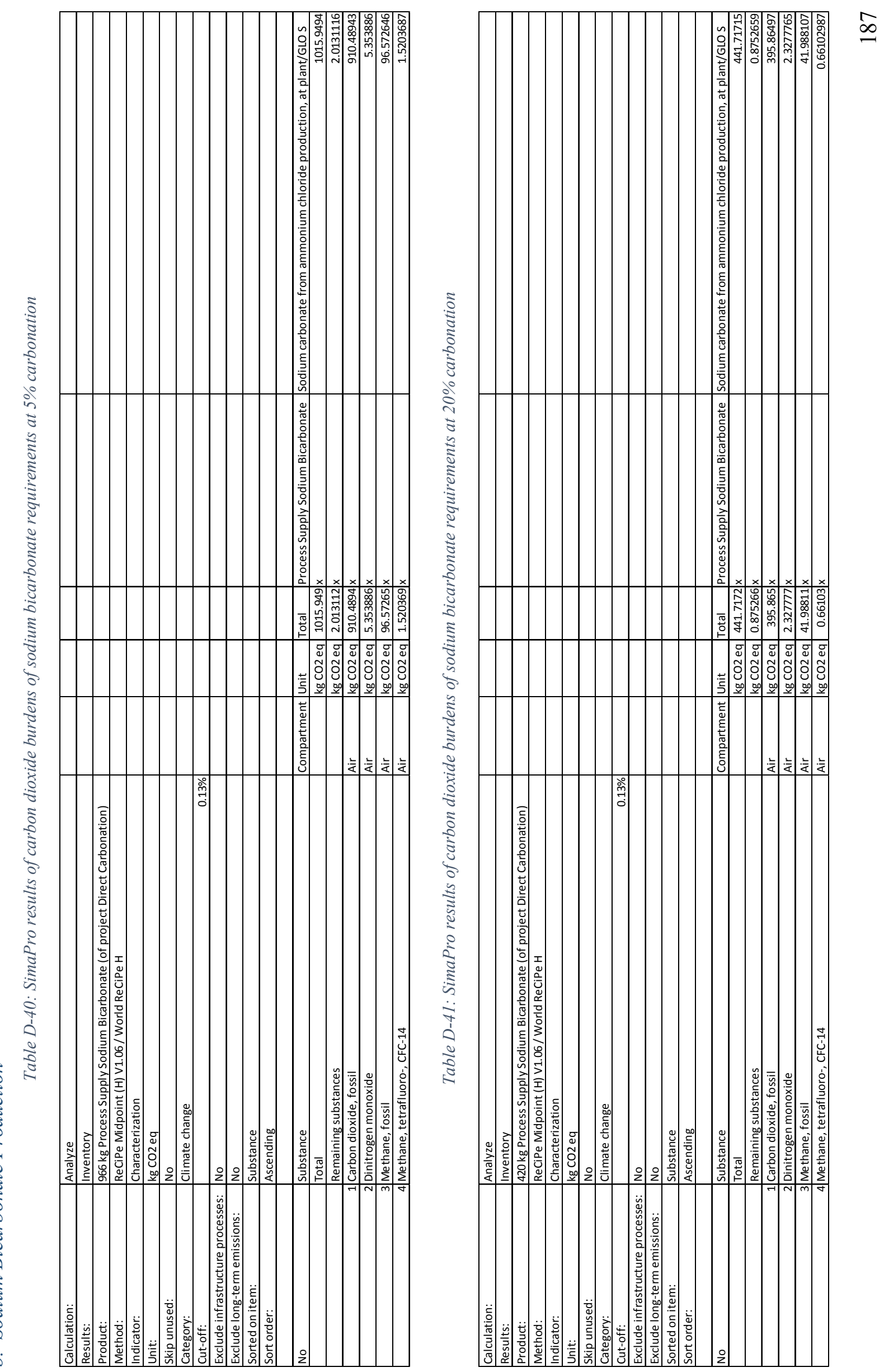
.
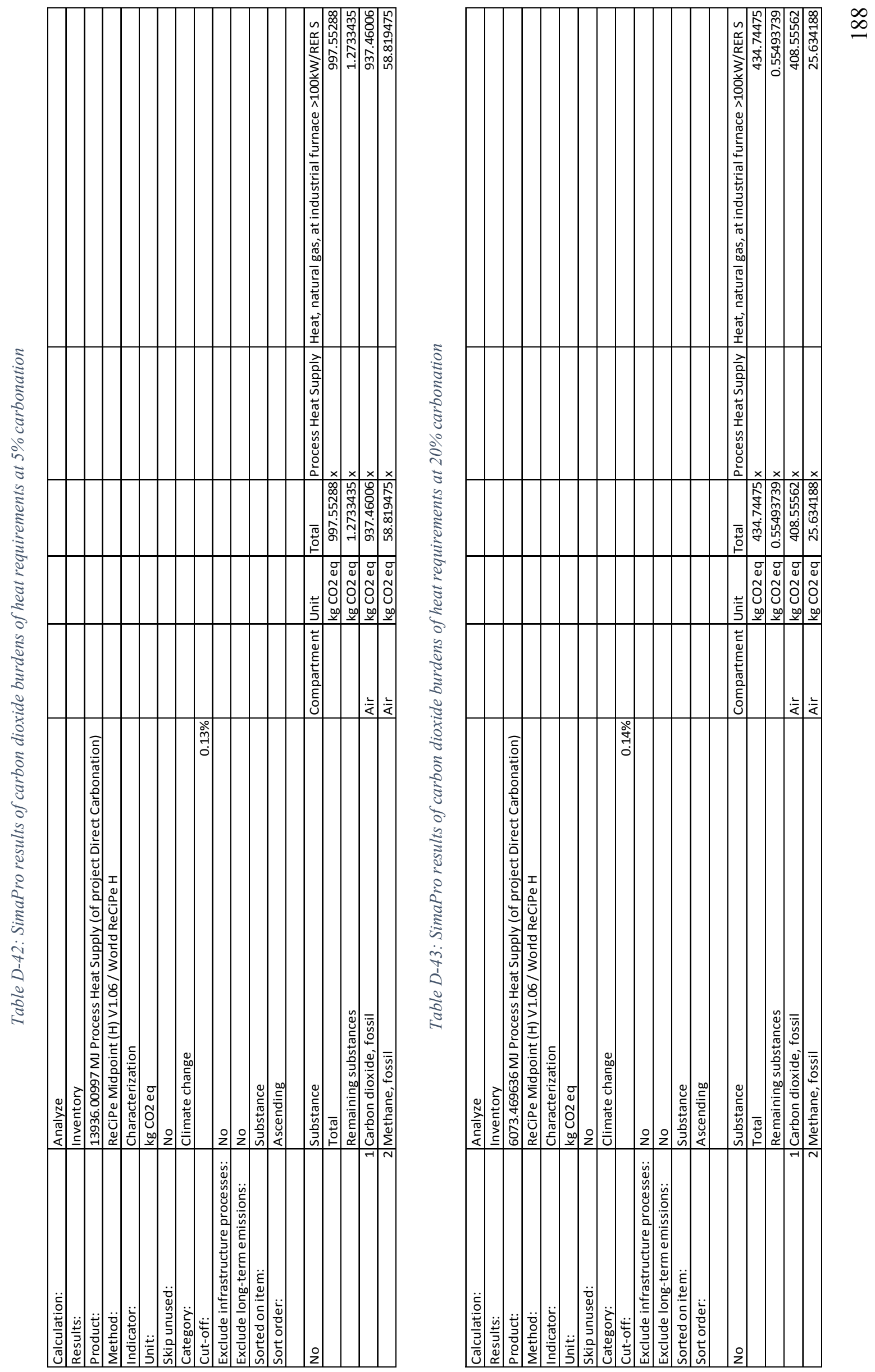
|ân


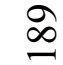




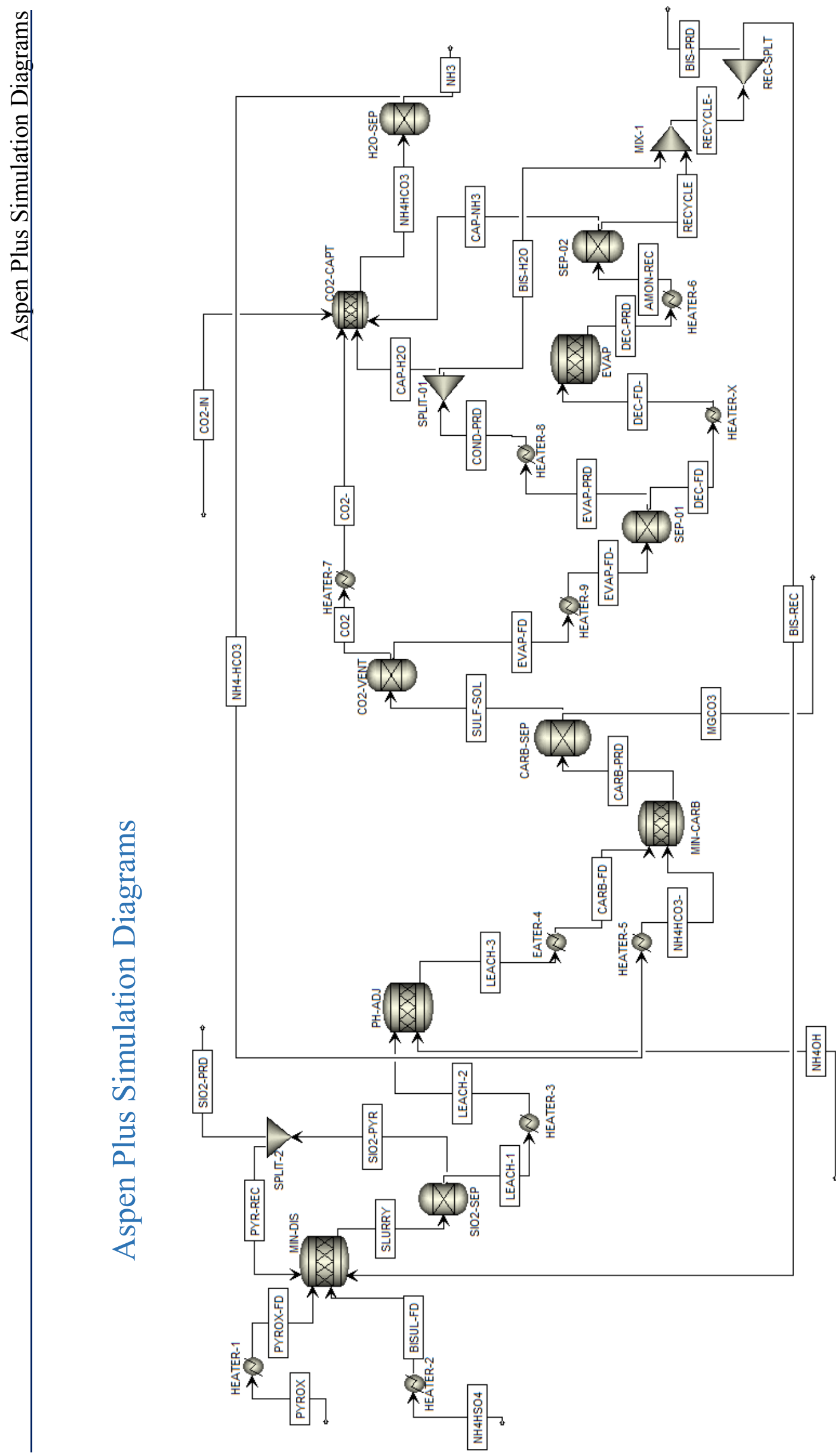

8 


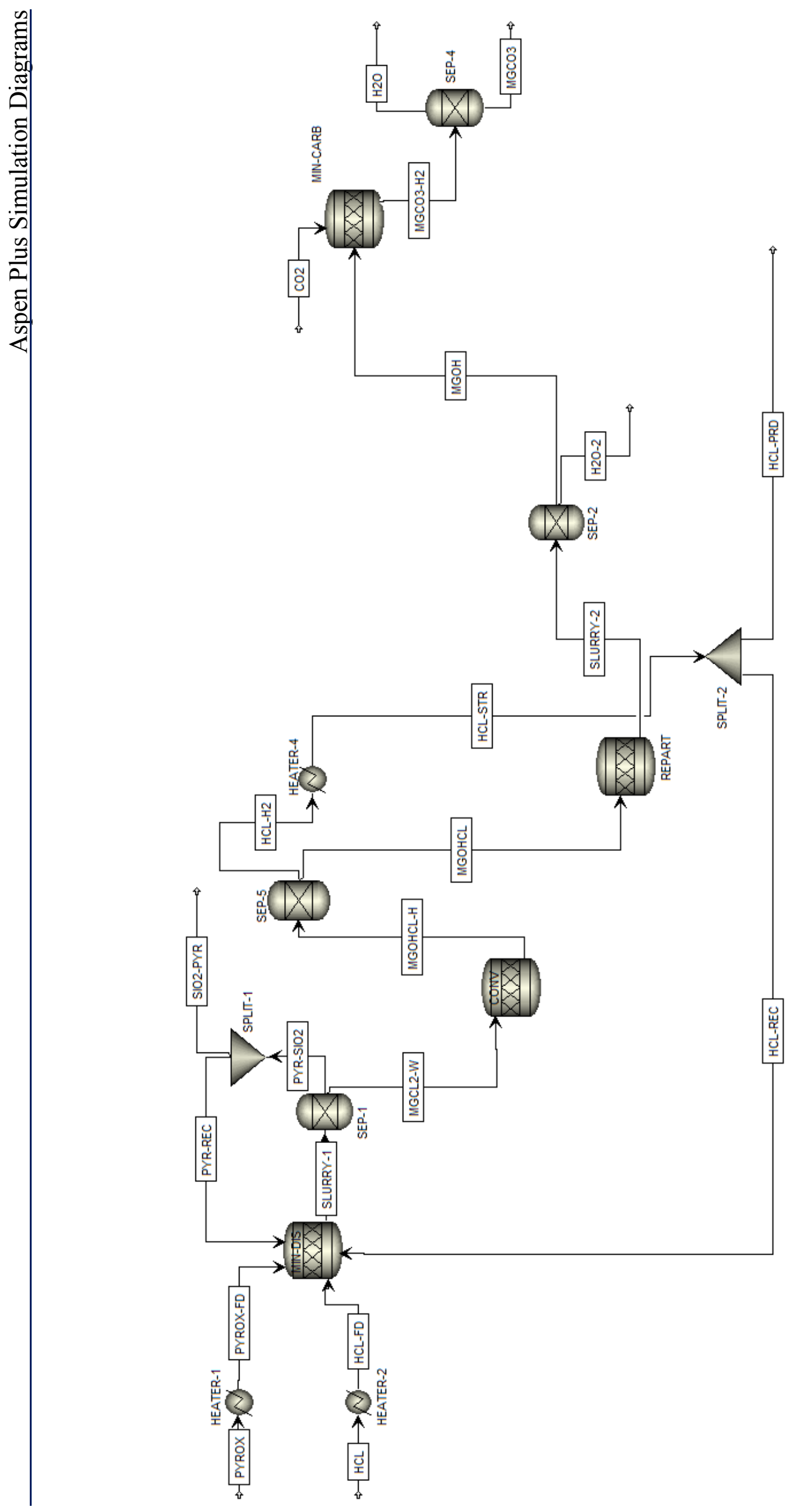




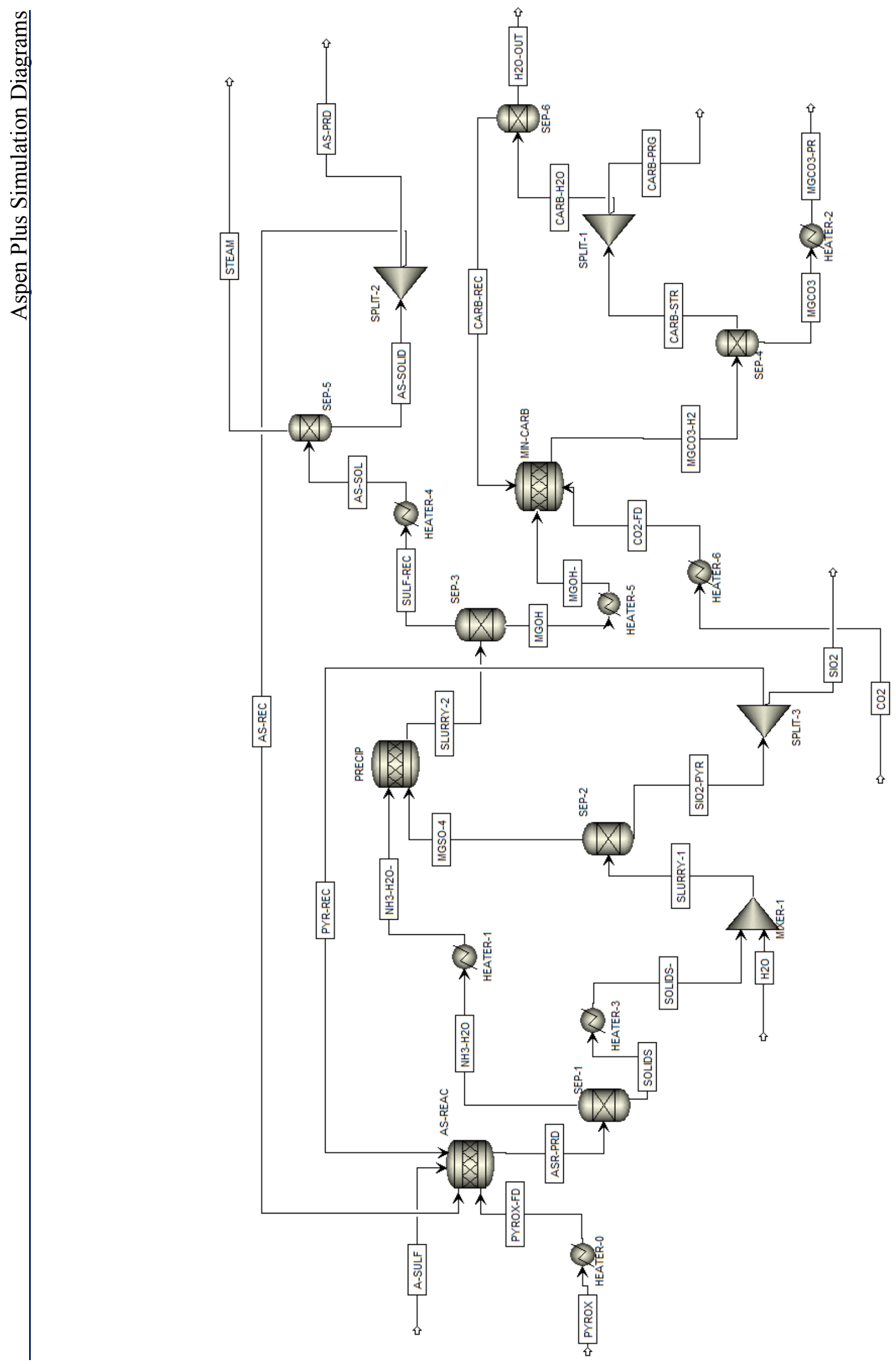

ธ 


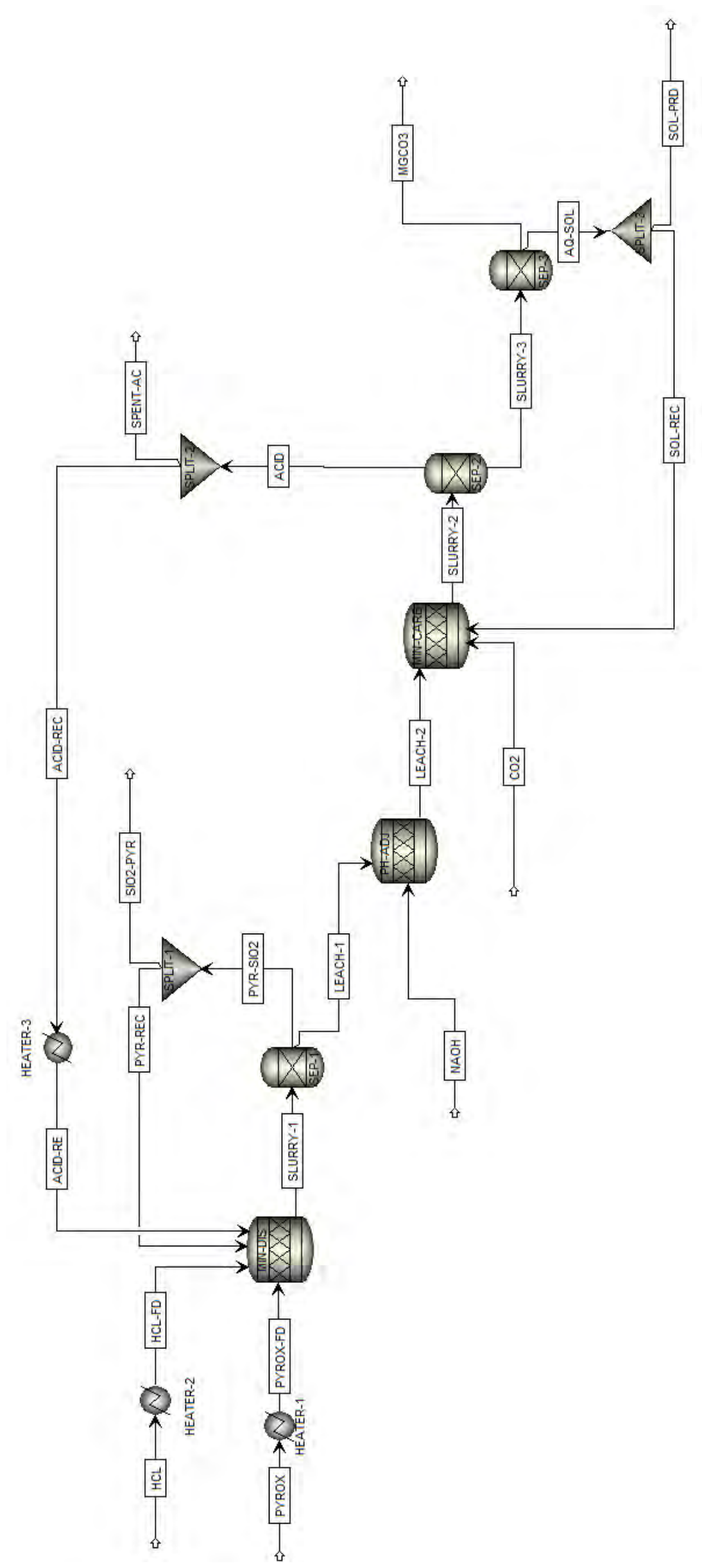

$\stackrel{2}{9}$ 

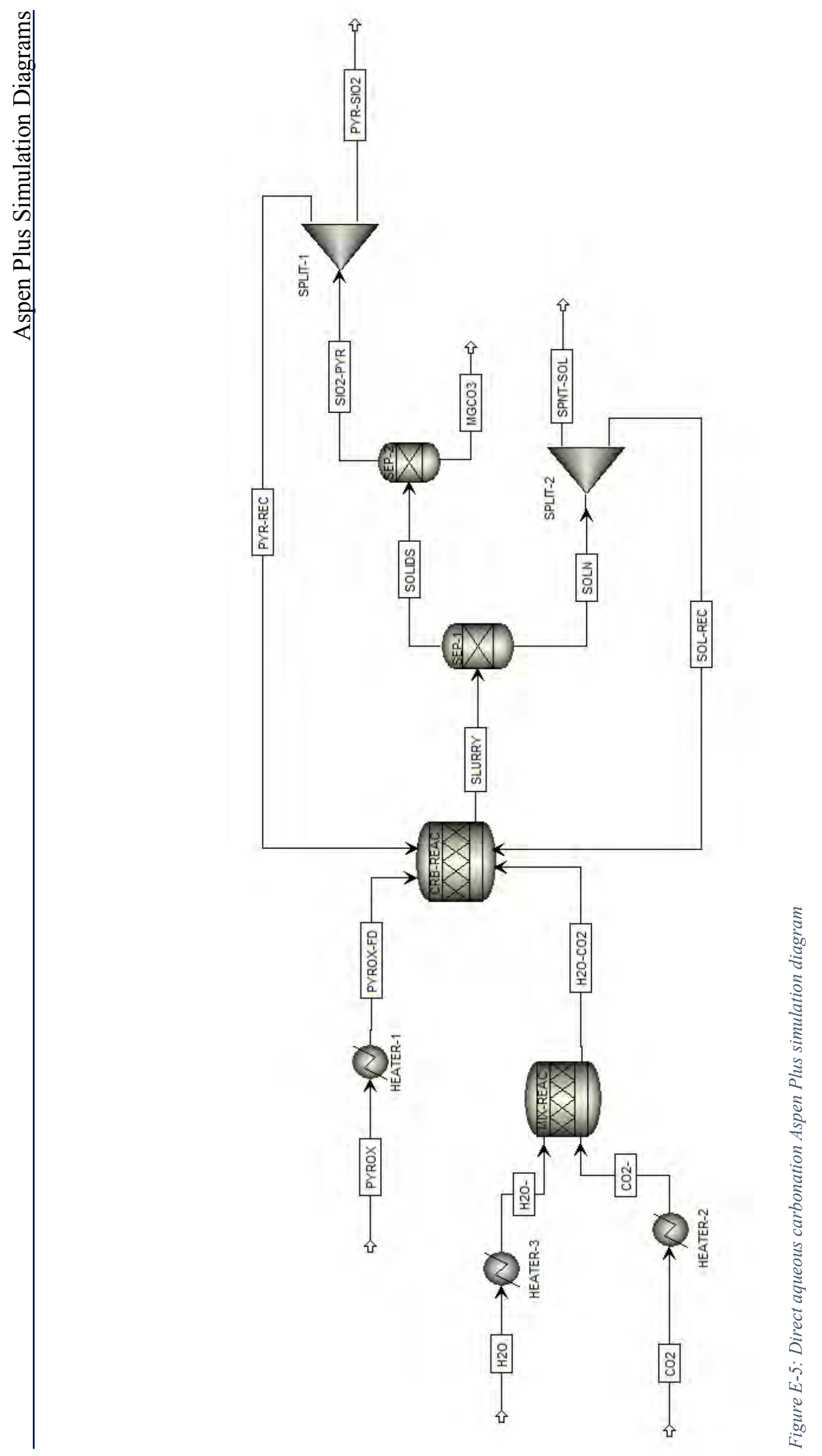


\section{Additional Supporting Data}

Table F-1: Net electricity requirements of selected mineral carbonation processes

\begin{tabular}{|l|c|c|c|}
\hline $\begin{array}{c}\text { Mineral Carbonation } \\
\text { Process }\end{array}$ & $\begin{array}{c}\text { Transport } \\
\text { Compression } \\
\text { (MJ/hr) }\end{array}$ & $\begin{array}{c}\text { Electricity } \\
\text { Credit } \\
\text { (MJ/hr) }\end{array}$ & $\begin{array}{c}\text { Net } \\
\text { Electricity } \\
\text { (MJ/hr) }\end{array}$ \\
\hline Ammonium Salts & 356.65 & -141.93 & 214.72 \\
\hline Lackner's Multi-stage $\mathrm{HCl}$ & 356.65 & -141.93 & 214.72 \\
\hline Abo Akademi & 356.65 & -57.20 & 299.45 \\
\hline Mineral Acid pH Swing & 356.65 & -141.93 & 214.72 \\
\hline Direct Aqueous & 356.65 & 50.89 & 407.53 \\
\hline
\end{tabular}

Table F-2: Process carbon dioxide emissions burdens and contributions

\begin{tabular}{|l|c|c|c|c|c|}
\hline \multirow{2}{*}{ Description } & \multicolumn{5}{|c|}{ Carbon Dioxide Emissions Burdens (kg-CO $\left.\mathrm{CO}_{2} \mathrm{e}\right)$} \\
\cline { 2 - 6 } & $\begin{array}{l}\text { Ammonium } \\
\text { Salts }\end{array}$ & $\begin{array}{l}\text { Lackner's } \\
\text { HCI Multi- } \\
\text { stage Process }\end{array}$ & $\begin{array}{l}\text { Abo } \\
\text { Akademi } \\
\text { University }\end{array}$ & $\begin{array}{l}\text { Mineral } \\
\text { Acid pH } \\
\text { Swing }\end{array}$ & $\begin{array}{l}\text { Direct } \\
\text { Aqueous }\end{array}$ \\
\hline Compression & 60 & 60 & 84 & 60 & 115 \\
\hline Heat Requirements & 7541 & 15677 & 1019 & 490 & 2116 \\
\hline Water & 84 & 163 & 6 & 36 & 264 \\
\hline Reagent Make-up & 1113 & 2394 & 232 & 570 & 2557 \\
\hline CO2 Released & 0 & 0 & 13 & 75 & 0 \\
\hline Total Burden & 8798 & 18295 & 1354 & 1231 & 5052 \\
\hline
\end{tabular}

Table F-3: The overall conversion of pyroxene in the selected mineral carbonation processes

\begin{tabular}{|c|c|c|}
\hline Selected Process & Single Pass Conversion & Overall Conversion \\
\hline \multirow{3}{*}{ Ammonium Salts } & $30 \%$ & $68 \%$ \\
\cline { 2 - 3 } & $50 \%$ & $83 \%$ \\
\cline { 2 - 3 } Lackner's Multi-Stage & $90 \%$ & $98 \%$ \\
\cline { 2 - 3 } HCl & $30 \%$ & $81 \%$ \\
\cline { 2 - 3 } & $50 \%$ & $91 \%$ \\
\hline \multirow{2}{*}{ AAU Process } & $90 \%$ & $99 \%$ \\
\cline { 2 - 3 } & $66 \%$ & $95 \%$ \\
\hline \multirow{2}{*}{ Mineral Acid pH } & $100 \%$ & $100 \%$ \\
\cline { 2 - 3 } Swing & $20 \%$ & $56 \%$ \\
\cline { 2 - 3 } & $30 \%$ & $68 \%$ \\
\hline \multirow{2}{*}{ Direct Aqueous } & $50 \%$ & $83 \%$ \\
\cline { 2 - 3 } & $5 \%$ & $26 \%$ \\
\hline
\end{tabular}

